\title{
Balanced and prepared
}

Citation for published version (APA):

Boots, L. (2016). Balanced and prepared: development and evaluation of a supportive e-health intervention for caregivers of people with early-stage dementia. [Doctoral Thesis, Maastricht University]. https://doi.org/10.26481/dis.20160701lb

Document status and date:

Published: 01/01/2016

DOI:

$10.26481 /$ dis.20160701lb

Document Version:

Publisher's PDF, also known as Version of record

\section{Please check the document version of this publication:}

- A submitted manuscript is the version of the article upon submission and before peer-review. There can be important differences between the submitted version and the official published version of record.

People interested in the research are advised to contact the author for the final version of the publication, or visit the DOI to the publisher's website.

- The final author version and the galley proof are versions of the publication after peer review.

- The final published version features the final layout of the paper including the volume, issue and page numbers.

Link to publication

\footnotetext{
General rights rights.

- You may freely distribute the URL identifying the publication in the public portal. please follow below link for the End User Agreement:

www.umlib.nl/taverne-license

Take down policy

If you believe that this document breaches copyright please contact us at:

repository@maastrichtuniversity.nl

providing details and we will investigate your claim.
}

Copyright and moral rights for the publications made accessible in the public portal are retained by the authors and/or other copyright owners and it is a condition of accessing publications that users recognise and abide by the legal requirements associated with these

- Users may download and print one copy of any publication from the public portal for the purpose of private study or research.

- You may not further distribute the material or use it for any profit-making activity or commercial gain

If the publication is distributed under the terms of Article $25 \mathrm{fa}$ of the Dutch Copyright Act, indicated by the "Taverne" license above, 


\section{BALANCED AND PREPARED}

Development and evaluation of a supportive e-health intervention for caregivers of people with early-stage dementia 
(C) Lizzy M.M. Boots, Maastricht 2016

Cover design: $\quad$ Stefan Fincken Design

Cover image: Jack Poels

Layout: Lizzy Boots

Printing: GildePrint

Publisher: $\quad$ NeuroPsych Publishers

All rights reserved. No part of this book may be reproduced or transmitted in any form or by any means, without prior permission in writing by the author, or when appropriate, by the publishers of the publication. 


\section{BALANCED AND PREPARED}

Development and evaluation of a supportive e-health intervention for caregivers of people with early-stage dementia

\section{PROEFSCHRIFT}

Ter verkrijging van de graad van doctor aan de Universiteit Maastricht, op gezag van de Rector Magnificus, Prof dr. L.L.G. Soete, volgens het besluit van het College van Decanen, in het openbaar te verdedigen op vrijdag

1 juli 2016 om 16.00 uur

door

\section{Lizzy Mitzy Maria Boots}

Geboren op 24 december 1988 te Roermond 


\section{Promotores}

Prof. dr. F.R.J. Verhey

Prof. dr. G.I.J.M. Kempen

\section{Copromotor}

Dr. M.E. de Vugt

\section{Beoordelingscommissie}

Prof. dr. C. van Heugten (voorzitter)

Prof. dr. A. Francke (NIVEL, VUmc)

Prof. dr. R. Ponds

Dr. E. van Rossum

Prof. dr. M. Vernooij-Dassen (Radboud UMC)

The research described in this thesis was performed at the School for Mental Health and Neuroscience, Department of Psychiatry and Neuropsychology, Maastricht University, the Netherlands and was sponsored by Alzheimer Nederland and the Alzheimer Onderzoeksfonds Limburg.

Printing of this thesis was kindly supported by Alzheimer Nederland and Danone Research - Centre for Specialised Nutrition.
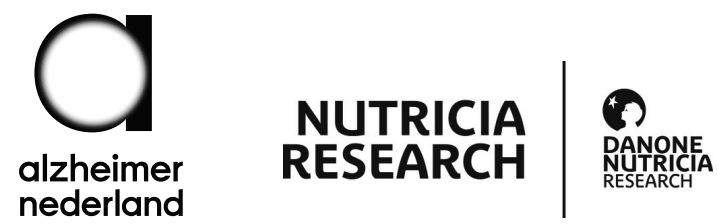
"Soms is 't beater iets moeis te verleeze. Beater verleeze dan dat ge 't noeit het gehad"

(Rowwen Hèze - Heilige Antonius)

Voor Opa en Oma 



\section{CONTENTS}

CHAPTER 1 General Introduction

CHAPTER 2 A systematic review of Internet-based supportive interventions for caregivers of patients with dementia International Journal of Geriatric Psychiatry, 2014

CHAPTER 3 A mismatch between demand and supply of social support in 55 dementia: a qualitative study on perspectives of spousal caregivers and their social network members Submitted

CHAPTER 4 Qualitative study on needs and wishes of early-stage dementia 79 caregivers: the paradox between needing and accepting help International Psychogeriatrics, 2015

CHAPTER 5 Development and initial evaluation of the web-based selfmanagement intervention 'Partner in Balance' for family caregivers of people with early-stage dementia: an exploratory mixed-methods study JMIR Research Protocols, 2016

CHAPTER 6 Effectiveness of the blended-care self-management program 'Partner in Balance' for early-stage dementia caregivers: study protocol for a randomized controlled trial Trials, 2016

CHAPTER 7 Process evaluation to explore internal and external validity of the blended care self-management program 'Partner in Balance' for early-stage dementia caregivers

CHAPTER 8 Effectiveness of the blended-care self-management program 183 'Partner in Balance' for early-stage dementia caregivers: results of a randomized controlled trial

CHAPTER 9 General Discussion

Summary

Samenvatting

Knowledge Valorization

Dankwoord

Curriculum Vitae

Publications

Thesis defenses from MHeNS 



\section{CHAPTER 1}

General Introduction 


\section{Dementia and Mild Cognitive Impairment}

Dementia is a generic term for deterioration in memory and other cognitive functions that facilitate a person's ability to function in everyday life. The most common cause of dementia is Alzheimer's disease, which is characterized by a slow and progressive course. ${ }^{1}$

Prior to a dementia diagnosis a transitional state of limited cognitive symptoms and preserved function abilities can occur, also known as Mild Cognitive Impairment $(\mathrm{MCl})$. While $\mathrm{MCl}$ is often regarded as the prodromal stage of dementia, a significant proportion of people with $\mathrm{MCl}$ remains stable or even improves. ${ }^{2}$ Nonetheless, people in the $\mathrm{MCl}$ stage must adapt to their cognitive problems and cope with the uncertainty about their future, which can trigger symptoms of depression and anxiety. $^{3}$

\section{Informal caregiver under pressure}

The majority of people with dementia is living at home and cared for by a family member, the informal caregiver. Informal care will be increasingly important as the number of people with dementia will rise to 65.7 million by 2030 and 115.4 million by 2050 , together with a decrease of the working population. ${ }^{4}$

However, informal caregiving has a downside. Caregivers of people with dementia are vulnerable due to the chronic stress they experience in the caregiving process. ${ }^{5}$ Caregivers have an increased risk of becoming depressed compared to non-caregivers, ${ }^{6}$ and $24 \%$ of caregivers report clinically significant levels of anxiety. ${ }^{7}$ Moreover, caregivers are susceptible to physical health problems as stress decreases their immune function and increases their disease vulnerability. ${ }^{8}$ Experiencing caregiver strain can even increase the mortality risk by $63 \% .{ }^{9,10}$

\section{Current caregiver interventions}

Many caregiver support interventions have been developed to ameliorate negative caregiver consequences. The most promising caregiver interventions focus on the mild to moderate stages of dementia and combine multiple components. Intervention elements that can be distinguished are: psycho-education, psychotherapy (primarily cognitive behavioral therapy), counseling, support groups, and respite care. A metaanalysis of high-quality randomized controlled trials (RCTs) showed promising effects of multicomponent caregiver interventions on delaying institutionalization of the person with dementia. ${ }^{11}$ 


\section{Caregiver functioning in different stages of the disease}

One of the essential elements in successful intervention programs is the focus on personal characteristics and resources. The efficacy of interventions increases if they are individualized to specific caregiver needs, competencies or characteristics, such as gender, age, educational background and relationship with the care recipient. ${ }^{12}$ Another aspect that might influence intervention effects is the stage of the disease, in which the role of the caregiver per stage can be considered as a career consisting of 3 phases: role acquisition or adaptation, role enactment, and role disengagement (Fig. 1). ${ }^{13,14}$

\section{CAREGIVER CAREER}

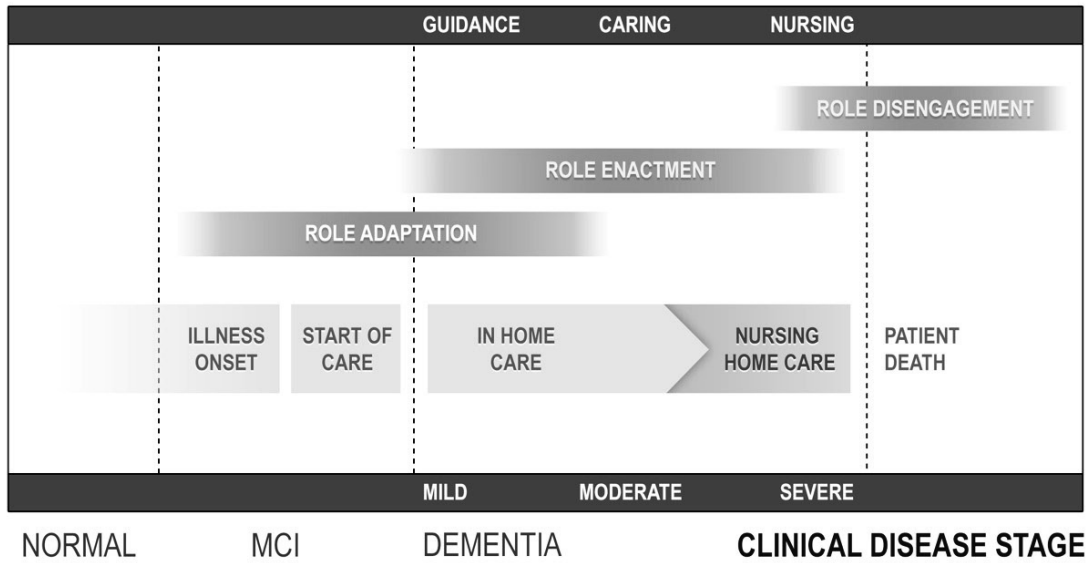

In the role adaptation phase caregivers have to learn to adapt to the changes in their role as family member and learn to cope with losses and occurring issues. Not all caregivers respond similarly to the different phases. Caregivers who use a supportive care strategy are best able to adapt to the changes; they supervise and support the care recipient's activities, communicate calmly or cautiously and try to comply with behavioral problems. ${ }^{5}$ On the other hand, non-adapting caregivers may respond impatiently, irritated or angrily. This confronting care strategy is portrayed by difficulties in understanding and accepting the situation, and increases the risk of depressive symptoms in the caregiver and hyperactivity in the person with dementia. ${ }^{5}$ As a reaction to experienced stressors, some caregivers seek social support and share stressful experiences, which contributes to improved caregiver well-being ${ }^{15,16}$, 
while others have low levels of social support, related to increased caregiver burden and depression. ${ }^{17,18}$ These differences in adaptation to the caregiver role and experienced stressors show that a diagnosis alone does not prepare caregivers for future care $^{14}$

\section{Early intervention}

The timing of caregiver interventions might be crucial in maximizing the effects. Early support might help people with dementia and their caregivers adapt to the changes of early dementia and feel more competent to care. However, the current contrast between highly advanced timely diagnostic tools and the lack of available support to match this early diagnosis is troubling. ${ }^{19}$ Efficacious pharmacotherapies are not yet available to cure cognitive disorders, but an early diagnosis offers opportunities to improve early adaptation to the caregiver role. In turn, this may prevent high levels of burden and institutionalization of the person with the dementia in the later stages.

Early support interventions can aim at coping with an insecure future and preparing caregivers for the possibility of further decline and dependency. ${ }^{14}$ Early intervention for caregivers proved to be effective in reducing strain and delaying institutionalization of the person with dementia. ${ }^{20,21}$ However, the evidence for earlystage interventions is not yet conclusive and they may do more harm than good if they do not fit the personal situation of the caregiver and care recipient. ${ }^{14}$ Taking action might cause a fear of stigma. $^{22}$ Interventions in the early phase should therefore not be problematizing, but may be particularly beneficial if they focus on adaptation to the caregiver role and actively involve caregivers to tailor the intervention to their needs. ${ }^{14}$

\section{Self-management}

Offering caregiver interventions in the pre- and early dementia stage asks for different approaches or skills. As levels of stress and burden are still low it provides more opportunities to empower the resources of caregivers.

Caregivers differ in their competence and skills to successfully manage dayto-day issues such as supervising or taking over daily tasks, managing cognitive problems, interpreting and managing behavioral changes, adapting to new social and economic circumstances, coping with relational and emotional consequences, and participating in care and treatment decisions. Those who feel that the demands of caring exceed their capacities will feel stressed, whereas those who feel equipped 
will not. ${ }^{23}$ The Stress and Coping paradigm by Lazarus and Folkman ${ }^{24}$ and the Social Learning theory by Bandura ${ }^{25}$ propose that taking charge of the changes in one's life has a positive effect on self-efficacy, and can therefore reduce caregiver stress and its negative impact on general wellbeing. ${ }^{26}$ Self-management programs provide an excellent opportunity to actively involve caregivers and let them choose the themes and strategies that are best individualized to their needs. This suits the caring role transition, which sometimes means focusing on positively managing life with dementia rather than managing the dementia itself. There is substantial evidence in other chronic diseases that individuals and families who engage in self-management behaviors improve their health outcomes and quality of life. ${ }^{27-29}$ Teaching selfmanagement techniques such as role management (e.g., balancing activities in daily life) and emotional management (e.g., dealing with fear and insecurity about the future) can foster self-care abilities and promote physical and mental wellbeing. ${ }^{30}$

\section{E-health technology to promote self-management}

Aside from intervention timing, intervention delivery can also be essential to maximize intervention effects. E-health technology offers an opportunity for accessible tailored interventions, which might fill the expected gap in demand and supply caused by demographic aging and a decreasing working population., ${ }^{1,31}$ Additionally, e-health applications may facilitate self-management, as sending and storing self-reports can significantly improve an individual's ability to understand and manage his or her own behaviors. ${ }^{32}$

Existing e-health programs for dementia caregivers are mainly aiming at dealing with dementia related problems such as neuropsychiatric symptoms in an advanced stage of the caregiver career. E-health self-management support could suit the early-stage facilitation to the adaptation process, by positively managing one's own life and increasing self-efficacy. It can also potentially bridge barriers to access support services for caregivers that would not utilize traditional healthcare services, due to for example practical reasons, refusal of the care recipient or fear of stigma. ${ }^{33-}$ 37

\section{Aim of the thesis}

The main aim of this thesis was to develop and evaluate an e-health selfmanagement intervention for caregivers of people with early-stage dementia. In this thesis we address the following research questions: 
1. What is the state of the art with respect to evidence of e-health interventions for caregivers of people with dementia?

2. What problems do caregivers of people with early-stage dementia experience and what are their needs for care and support?

3. How should an e-health self-management program for caregivers of people with early-stage dementia look like and to what extent is such a program feasible?

4. What are the process characteristics and effects of the blended-care selfmanagement program 'Partner in Balance' on self-efficacy, mastery, and psychological complaints (stress, depression, anxiety)?

\section{Outline of the thesis}

In Chapter 2 an overview is provided of the evidence for the effectiveness, feasibility, and quality of studies that focus on Internet interventions for dementia caregivers.

In Chapter 3 we explore primary caregiver and social network members' needs, perceptions and experiences with respect to informal support.

In Chapter 4 an in-depth analysis of the problems, needs and wishes of caregivers of people with early-stage dementia and their perspective on need for care during these early stages is provided.

Chapter 5 describes the development of an e-health self-management program for caregivers of people with early-stage dementia and the evaluation of its feasibility and preliminary effectiveness on self-efficacy and goal attainment.

In Chapter 6 the design of a randomized waiting-list controlled trial investigating the effects and process of the blended-care self-management program 'Partner in Balance' is presented.

Chapter 7 assesses the internal and external validity of the 'Partner in Balance' program and trial and its implementation in a process evaluation. 
Chapter 8 investigates if 'Partner in Balance' is superior to the waiting-list control condition as evidenced by improved subjective self-confidence (self-efficacy and mastery), and lower levels of psychological complaints (depression, anxiety, and stress).

In Chapter 9 the main results are discussed together with a reflection on methodological issues and the clinical and scientific implications of our studies. 


\section{References}

1. Association As. Alzheimer's Disease Facts and Figures. Alzheimers Dement 2014; 10(2).

2. Mitchell AJ, Shiri-Feshki M. Rate of progression of mild cognitive impairment to dementia--meta-analysis of 41 robust inception cohort studies. Acta Psychiatr Scand 2009; 119(4): 252-65.

3. Ramakers IH, Visser PJ, Aalten P, Kester A, Jolles J, Verhey FR. Affective symptoms as predictors of Alzheimer's disease in subjects with mild cognitive impairment: a 10-year follow-up study. Psychol Med 2010; 40(7): 1193-201.

4. Alzheimer's Disease International. Policy Brief for Heads of Government: The Global Impact of Dementia 2013-2050. London: ADI, 2013.

5. de Vugt ME, Stevens F, Aalten P, et al. Do caregiver management strategies influence patient behaviour in dementia? Int J Geriatr Psychiatry 2004; 19(1): 85-92.

6. Joling $\mathrm{KJ}$, van Hout HP, Schellevis FG, et al. Incidence of depression and anxiety in the spouses of patients with dementia: a naturalistic cohort study of recorded morbidity with a 6-year follow-up. Am J Geriatr Psychiatry 2010; 18(2): 146-53.

7. Mahoney R, Regan C, Katona C, Livingston G. Anxiety and depression in family caregivers of people with Alzheimer disease: the LASER-AD study. Am J Geriatr Psychiatry 2005; 13(9): 795-801.

8. Bauer ME, Vedhara K, Perks P, Wilcock GK, Lightman SL, Shanks N. Chronic stress in caregivers of dementia patients is associated with reduced lymphocyte sensitivity to glucocorticoids. J Neuroimmunol 2000; 103(1): 8492.

9. Schulz R, Beach SR. Caregiving as a risk factor for mortality: the Caregiver Health Effects Study. Jama 1999; 282(23): 2215-9.

10. Perkins M, Howard VJ, Wadley VG, et al. Caregiving strain and all-cause mortality: evidence from the REGARDS study. J Gerontol B Psychol Sci Soc Sci 2013; 68(4): 504-12.

11. Olazaran J, Reisberg B, Clare L, et al. Nonpharmacological therapies in Alzheimer's disease: a systematic review of efficacy. Dement Geriatr Cogn Disord 2010; 30(2): 161-78.

12. Sorensen $S$, Pinquart $M$, Duberstein $P$. How effective are interventions with caregivers? An updated meta-analysis. Gerontologist 2002; 42(3): 356-72. 
13. Aneshensel CS, Pearlin LI, Mullan JT, Zarit SH, Whitlatch CJ. Profiles in Caregiving; The Unexpected Career. San Diego: Academic Press Inc; 1995.

14. de Vugt ME, Verhey FR. The impact of early dementia diagnosis and intervention on informal caregivers. Prog Neurobiol 2013; 110: 54-62.

15. Au A, Lai M-K, Lau K-M, et al. Social support and well-being in dementia family caregivers: The mediating role of self-efficacy. Aging \& Mental Health 2009; 13(5): 761-8.

16. Kiecolt-Glaser JK, Dyer CS, Shuttleworth EC. Upsetting social interactions and distress among Alzheimer's disease family care-givers: a replication and extension. Am J Community Psychol 1988; 16(6): 825-37.

17. Coen RF, O'Boyle CA, Coakley D, Lawlor BA. Dementia carer education and patient behaviour disturbance. Int J Geriatr Psychiatry 1999; 14(4): 302-6.

18. Gold DP, Cohen C, Shulman K, Zucchero C, Andres D, Etezadi J. Caregiving and dementia: predicting negative and positive outcomes for caregivers. Int $J$ Aging Hum Dev 1995; 41(3): 183-201.

19. Verhey $F$, de Vugt $M$. Mild cognitive impairment is becoming more psychosocial. Aging Ment Health 2013; 17(3): 265-6.

20. Gaugler JE, Kane RL, Kane RA, Newcomer R. Early community-based service utilization and its effects on institutionalization in dementia caregiving. Gerontologist 2005; 45(2): 177-85.

21. Kjallman Alm A, Hellzen O, Norbergh KG. Experiences of long term ongoing structured support in early stage of dementia - a case study. Int $J$ Older People Nurs 2013.

22. Roelands $M$, Van Oost $P$, Depoorter A. Service use in family caregivers of persons with dementia in Belgium: psychological and social factors. Health Soc Care Community 2008; 16(1): 42-53.

23. Donaldson C, Tarrier N, Burns A. Determinants of carer stress in Alzheimer's disease. Int J Geriatr Psychiatry 1998; 13(4): 248-56.

24. Lazarus R, Folkman S. Stress, appraisal and coping. New York: Springer; 1984.

25. A. B. Self-Efficacy: The Exercise of Control. . New York: W.H. Freeman and Company; 1997.

26. Acton GJ, Kang J. Interventions to reduce the burden of caregiving for an adult with dementia: a meta-analysis. Res Nurs Health 2001; 24(5): 349-60. 
27. Ryan P, Sawin KJ. The Individual and Family Self-Management Theory: background and perspectives on context, process, and outcomes. Nurs Outlook 2009; 57(4): 217-25 e6.

28. Lorig KR, Ritter PL, Dost A, Plant K, Laurent DD, McNeil I. The Expert Patients Programme online, a 1-year study of an Internet-based selfmanagement programme for people with long-term conditions. Chronic IIIn 2008; 4(4): 247-56.

29. Forducey PG, Glueckauf RL, Bergquist TF, Maheu MM, Yutsis M. Telehealth for persons with severe functional disabilities and their caregivers: facilitating self-care management in the home setting. Psychol Serv 2012; 9(2): 144-62.

30. Lorig KR, Holman $H$. Self-management education: history, definition, outcomes, and mechanisms. Ann Behav Med 2003; 26(1): 1-7.

31. Alzheimer Disease International. World Alzheimer Report. London: ADI, 2010.

32. Ramanathan N, Swendeman D, Comulada WS, Estrin D, Rotheram-Borus MJ. Identifying preferences for mobile health applications for self-monitoring and self-management: focus group findings from HIV-positive persons and young mothers. Int J Med Inform 2013; 82(4): e38-46.

33. Brodaty $\mathrm{H}$, Thomson $\mathrm{C}$, Thompson $\mathrm{C}$, Fine $\mathrm{M}$. Why caregivers of people with dementia and memory loss don't use services. Int J Geriatr Psychiatry 2005; 20(6): 537-46.

34. Wolfs CA, de Vugt ME, Verkaaik M, Verkade PJ, Verhey FR. Empowered or overpowered? Service use, needs, wants and demands in elderly patients with cognitive impairments. International journal of geriatric psychiatry 2010; 25(10): 1006-12.

35. Wolfs CA, de Vugt ME, Verkaaik M, et al. Rational decision-making about treatment and care in dementia: A contradiction in terms? Patient Educ Couns 2011.

36. Serafini JD, Damianakis T, Marziali E. Clinical practice standards and ethical issues applied to a virtual group intervention for spousal caregivers of people with Alzheimer's. Soc Work Health Care 2007; 44(3): 225-43.

37. Davis Kirsch SE, Lewis FM. Using the World Wide Web in Health-related intervention research. CIN: Computers, Informatics, Nursing 2004; 22(1): 8-18. 




\section{CHAPTER 2}

\section{A systematic review of Internet-based supportive interventions for caregivers of patients with dementia}

International Journal of Geriatric Psychiatry, 2014

Lizzy M.M. Boots, Marjolein E. de Vugt, Rosalie J.M. van Knippenberg, Gertrudis I.J.M. Kempen \& Frans R.J. Verhey 


\section{ABSTRACT}

Objective: Due to the expected increase in the number dementia patients, the unlikelihood of a cure in the near future, and the rising cost of care, there is an increasing need for effective caregiver interventions. Internet interventions hold considerable promise for meeting the educational and support needs of informal dementia caregivers at reduced costs. The current study aims to provide an overview of the evidence for the effectiveness, feasibility, and quality of Internet interventions for informal caregivers of people with dementia.

Methods: A systematic literature search of five scientific databases was performed, covering literature published up to January $10^{\text {th }}, 2013$. Twelve studies were identified. The quality of the included studies was assessed according to the Cochrane Level of Evidence (LOE) and the criteria list of the Cochrane Back Review Group.

Results: The intervention types, dosage and duration differed widely, as did the methodological quality of the included studies. The overall level of evidence was low. However, the results demonstrate that Internet interventions for informal dementia caregivers can improve various aspects of caregiver well-being, e.g., confidence, depression and self-efficacy, provided they comprise multiple components and are tailored to the individual. Furthermore, caregivers could benefit from interaction with a coach and other caregivers.

Conclusions: Internet interventions for informal dementia caregivers may improve caregiver well-being. However, the available supporting evidence lacks methodological quality. More randomized controlled studies assessing interventions performed according to protocol are needed to give stronger statements about the effects of supportive Internet interventions and their most promising elements. 


\section{INTRODUCTION}

Worldwide, 36 million people live with dementia, and this number is expected to increase to an alarming 115.4 million by 2050 . The global cost of dementia was estimated at 604 billion dollars in $2010 .^{1}$ Due to the major increase in the number of dementia patients in the near future, it is expected that the cost of care for dementia will increase by $85 \%$ in 2030 , possibly making it the most expensive disease of our society. ${ }^{1}$

Most patients with dementia live at home and are cared for primarily by their spouse or other family member, the informal caregiver. These informal caregivers often provide years of extensive care for their loved one and encounter continuously changing and demanding situations. ${ }^{2}$ Caregivers of dementia patients often suffer from physical and emotional problems as a result of the stressful and burdensome caregiving process. ${ }^{2-4}$ Therefore, they could be referred to - as Brodaty and Donkin ${ }^{5}$ put it - "the invisible second patient".

Due to the expected increase in the number dementia patients, the unlikelihood of a cure in the near future, and the rising cost of care, there is a growing need for effective caregiver interventions to adequately support the informal caregiver. Many psychosocial interventions have been developed to offer support for informal caregivers of patients with dementia ${ }^{6,7}$ and these interventions have demonstrated great potential in improving caregiver mood, improving caregiver quality of life and delaying the institutionalization of the care recipient. Olazaran and colleagues $^{8}$ found that these interventions can delay institutionalization for up to 1.5 years. These results are noteworthy, considering that a single day at a nursing home in the USA costs approximately 200 Dollars. ${ }^{9}$ Therefore, interventions aimed at reducing care burden and depression and at increasing feelings of competence among informal caregivers might reduce dementia care costs.

Most currently available interventions are 'face-to-face' interventions, but the predicted increase in the number of people with dementia has led to concerns about whether the current labor force can cope with such an increased future demand. ${ }^{10} \mathrm{E}-$ health interventions could be an efficient alternative to continue to provide education and support for the informal caregivers ${ }^{11,12}$ at reduced costs. ${ }^{13}$ In addition, interventions offered via the Internet likely exhibit a lower threshold of access for participation since informal caregivers can access help in the privacy of their own homes without leaving their loved ones alone. Technology can also reach people who are isolated and have difficulties accessing traditional services. ${ }^{14}$ Internet 
interventions might help future informal caregivers cope with the challenges of caring for a person with dementia and, as a result, may keep patients at home as long as possible. $^{15}$

This systematic review aims to assess the effectiveness and feasibility of Internet-based interventions for informal caregivers of people with dementia and to evaluate if such interventions indeed improve caregivers' well-being. Previous reviews $^{16,17}$ that have discussed technology supporting the needs of people with dementia and their caregivers only covered literature up to February 2007. In addition, such studies focused more on assistive technologies for patients and formal caregivers ${ }^{16}$ or compared technology-based interventions with non-technology-based interventions. ${ }^{17}$ The rapid growth of older adults using the Internet in the last few years ${ }^{18}$ underlines the importance of investigating Internet interventions as a source of support for this group. The current review aims to address the following objectives: (I) to present an overview of the evidence of the effectiveness and quality of Internet interventions for the informal caregivers of patients with dementia; (II) to assess which types of interventions are most effective (i.e., provide the best outcome for the participants); and (III) to indicate the feasibility of such interventions.

\section{METHODS}

\section{Search strategy}

A systematic literature search was conducted in PubMed, Psychinfo, Cinahl, Web of Science and the Cochrane Library as well as searches of references listed in the reviewed papers and meta-analyses. The search was restricted to effect studies on Internet-based interventions for informal caregivers (e.g., spouses/children/childrenin-law) of individuals with dementia or Mild Cognitive Impairment (MCl). For practical reasons, the search was limited to papers written in English and Dutch. We used MESH and Thesaurus terms, in addition to free text words, covering all studies published between January 1988 (start search engines we used, and Web of Science) and January 2013. For an overview of the search terms that were used, see Box 1. 


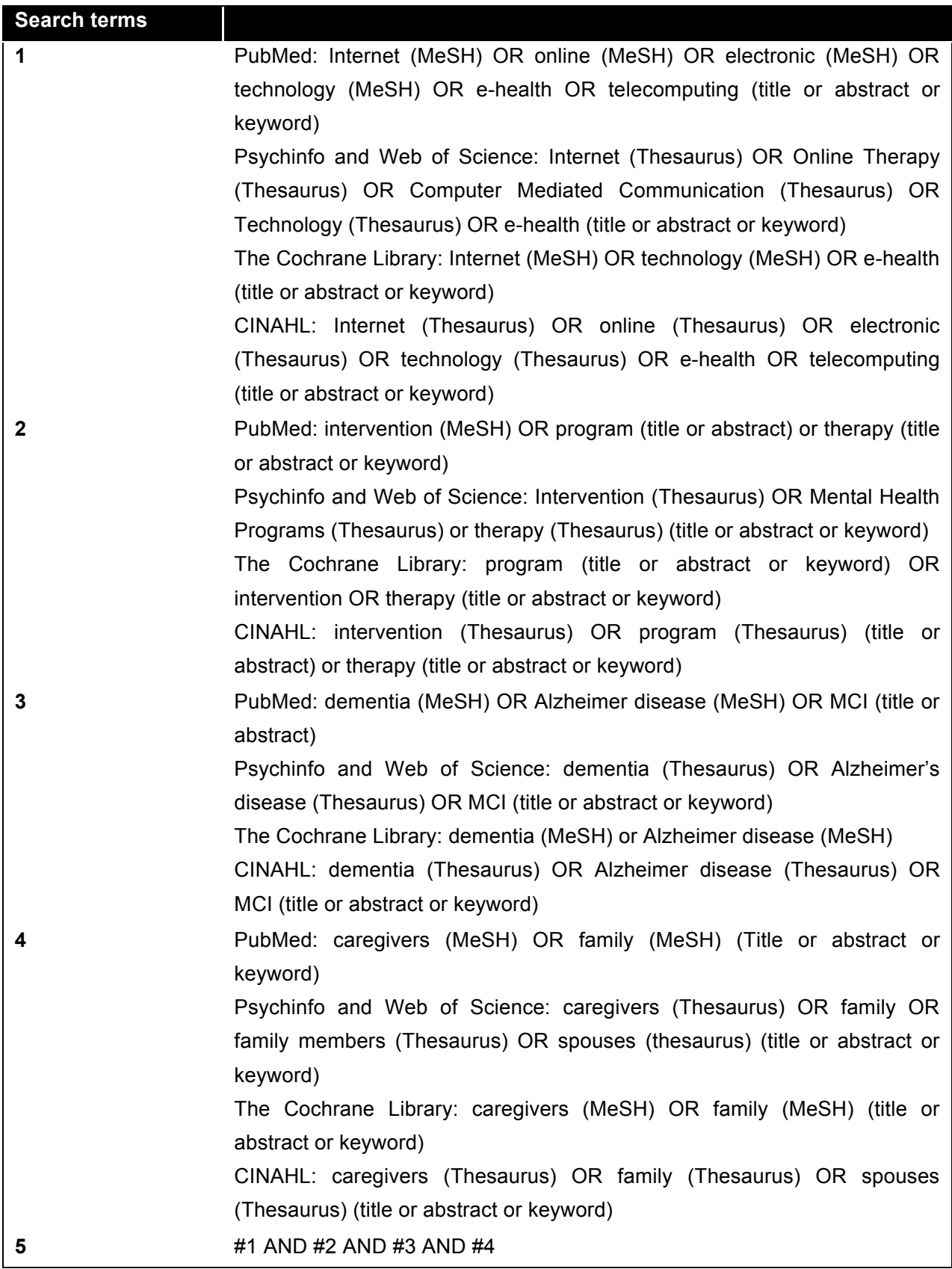




\section{Selection of studies}

The papers selected for this review met the following criteria: (1) reported the effects (quantitative and qualitative) of an intervention; (2) were Internet-based; and (3) were aimed at informal (non-professional) caregivers of (4) patients with $\mathrm{MCl} /$ dementia. No methodological quality restrictions were imposed. Papers were excluded if the intervention was solely aimed at the patient instead of the caregiver. Based on the above criteria, the titles and abstracts of the obtained studies were assessed by two authors (LB, MdV). When the title and abstract did not provide sufficient information concerning the criteria, full copies of the articles were retrieved and screened. The corresponding authors of the studies were contacted when additional information was required to assess whether the studies met the criteria for inclusion. Two reviewers (LB, RvK) screened the full copies of the selected studies for their eligibility. Any disagreement about the inclusion of papers was discussed in a consensus meeting with a third reviewer (MdV). Figure 1 presents a flowchart illustrating the selection process. The initial search resulted in a total of 219 intervention studies, of which 49 met the inclusion criteria. These studies were further analyzed and resulted in nine studies that described Internet-based interventions aimed at informal dementia caregivers. All reviewers (LB, RvK, MdV) checked the reference lists, which resulted in the inclusion of three additional articles.

\section{Quality assessment}

To assess the methodological quality of the included trials, we used the criteria list of the Cochrane Back Review Group ${ }^{19}$ to specifically rate descriptive, statistical and internal validity aspects. Because it is impossible in psychosocial intervention trials to blind the therapists to the intervention, this item was not included as one of the quality criteria, but blinding of outcome assessors was included. Scores on validity items theoretically ranged from zero to nine per study. Each item was scored positive $(+)$, negative (-), or undefined (?) if information was not provided or was unclear. Studies were considered to be of "higher methodological quality" if at least four items scored positively. ${ }^{20}$ In addition to the criteria of the Cochrane Back Review Group, we used the level of evidence (LOE) grade according to the Oxford Centre for EvidenceBased Medicine guidelines ${ }^{21}$ to broadly categorize the study designs of the selected articles displayed in Table 1. The LOE rates studies based on their design, proposing a hierarchy of study designs in ascending order of bias control. 


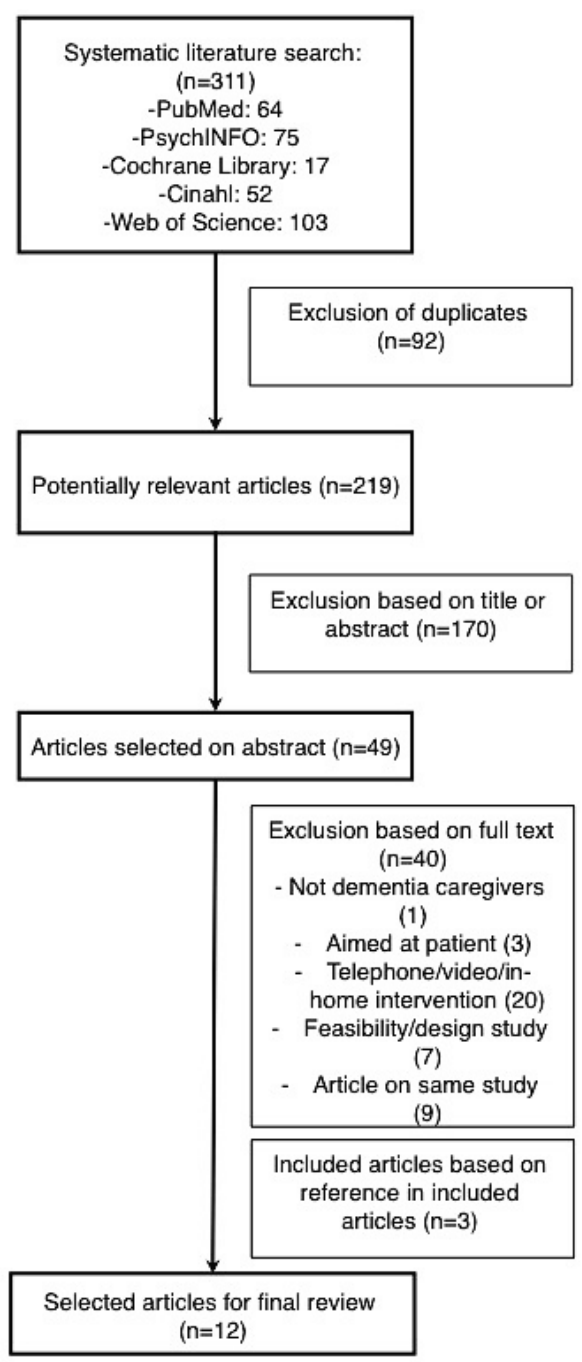

Figure 1. Flowchart of The Search Strategy

For instance, the presence of randomization, prospective follow-up and replication of evidence raises the ranking of a study within the hierarchy and thereby the level of evidence. Level one is the highest level of evidence, including high-quality systematic reviews; level five is the lowest level of evidence, including the so-called expert opinion. The grading system is widely accepted and often used to apply weights to individual studies according to their methodological quality ${ }^{22-24}$. Finally, the process characteristics of the interventions were reviewed by checking whether the 
intervention studies provided information on the following: (a) the characteristics of the facilitator; (b) the compliance of participants with the intervention; (c) the dropout rate during the intervention; (d) the reasons for dropout during the intervention; (e) the performance of intervention according to its protocol; (f) the feasibility of the intervention; and $(\mathrm{g})$ recommendations for improving the intervention. A similar approach was used by Zijlstra and colleagues ${ }^{25}$.

Two authors (LB, RvK) independently reviewed the methodological quality of the included studies. Disagreement between the researchers was resolved in a consensus meeting together with the second author (MdV), who gave the decisive judgment.

\section{Data extraction}

Relevant studies were examined for their study characteristics, participant characteristics, setting and content of the intervention, methodological quality, process characteristics and the outcome data or results found. To this end, we used a standardized data extraction form, as suggested by the Cochrane Handbook for Systematic Reviews of Interventions ${ }^{26}$. Because the studies were expected to be heterogeneous in terms of design, sample sizes, methods, and interventions (Table 1), we decided not to statistically pool the data to perform a quantitative metaanalysis.

\section{RESULTS}

\section{Study characteristics}

The search resulted in 12 articles that were included for review. All studies were aimed at improving informal caregiver functioning; however, the selected studies were heterogeneous in various aspects (Table 1). The design of the studies differed substantially, as did the number of study samples, ranging from $11^{27}$ to $700 .^{28}$ Table 1 shows a more detailed description of the participants. In addition, the studies differed in terms of study duration, setting and intervention characteristics. The following intervention types were identified: a website with information and support on various aspects of caregiving, ${ }^{28-35}$ a website with additional caregiving strategies, ${ }^{36}$ a website combined with telephone support, ${ }^{37}$ a website with additional e-mail support, $^{28,29}$ and a website with a combination of individual work and exchange with other caregivers online. ${ }^{27,28,30-32,35}$ 
In four of the 12 interventions the program was tailored to the individual needs of the informal caregiver, ${ }^{30,34,37,38}$ and in two of these studies, participants were guided by a coach. ${ }^{30,37}$ Five studies included a control group. Control conditions consisted of care as usual, ${ }^{34,35}$ waitlist controls, ${ }^{38}$ an onsite workshop conducted in seven weekly faceto-face sessions, ${ }^{27}$ and placebo online training to identify local services and resources. ${ }^{33}$ The dosage and duration of the interventions varied greatly; some intervention programs were available for a longer period of time, but the actual time spent online was not mentioned or limited, ${ }^{28,33,34,38}$ whereas others ${ }^{27,30,37}$ comprised a fixed intervention dosage in the form of weekly sessions. All studies focused on more than one caregiver functioning-related outcome. The most commonly addressed topics included self-efficacy, stress/burden, depressive symptoms, coping, social contact/support, knowledge, utilization of health services, and general (mental) health. A few included studies focused on additional topics, including positive aspects of caregiving, needs met or goals attained and caregivers' quality of life.

\section{Validity and methodology}

The second-to-last column of Table 1 contains the level of evidence (LOE) as graded by the Oxford Centre of Evidence-Based Medicine. ${ }^{21}$ The LOE for the studies included in this review ranged between two and three. Three papers ${ }^{27,33,38}$ received an evidence score of two. Most studies $(n=9)$ exhibited level three evidence. Of the 12 selected studies, three were randomized controlled trials, ${ }^{27,33,38}$ four used mixed methods designs with qualitative and quantitative measures, ${ }^{28-30,32}$ and one made use of a single-group pretest-posttest design. ${ }^{37}$ There was one quasi-experimental study, ${ }^{31}$ two pretest-posttest multiple groups designs, ${ }^{34,35}$ and one formative evaluation. $^{36}$ 


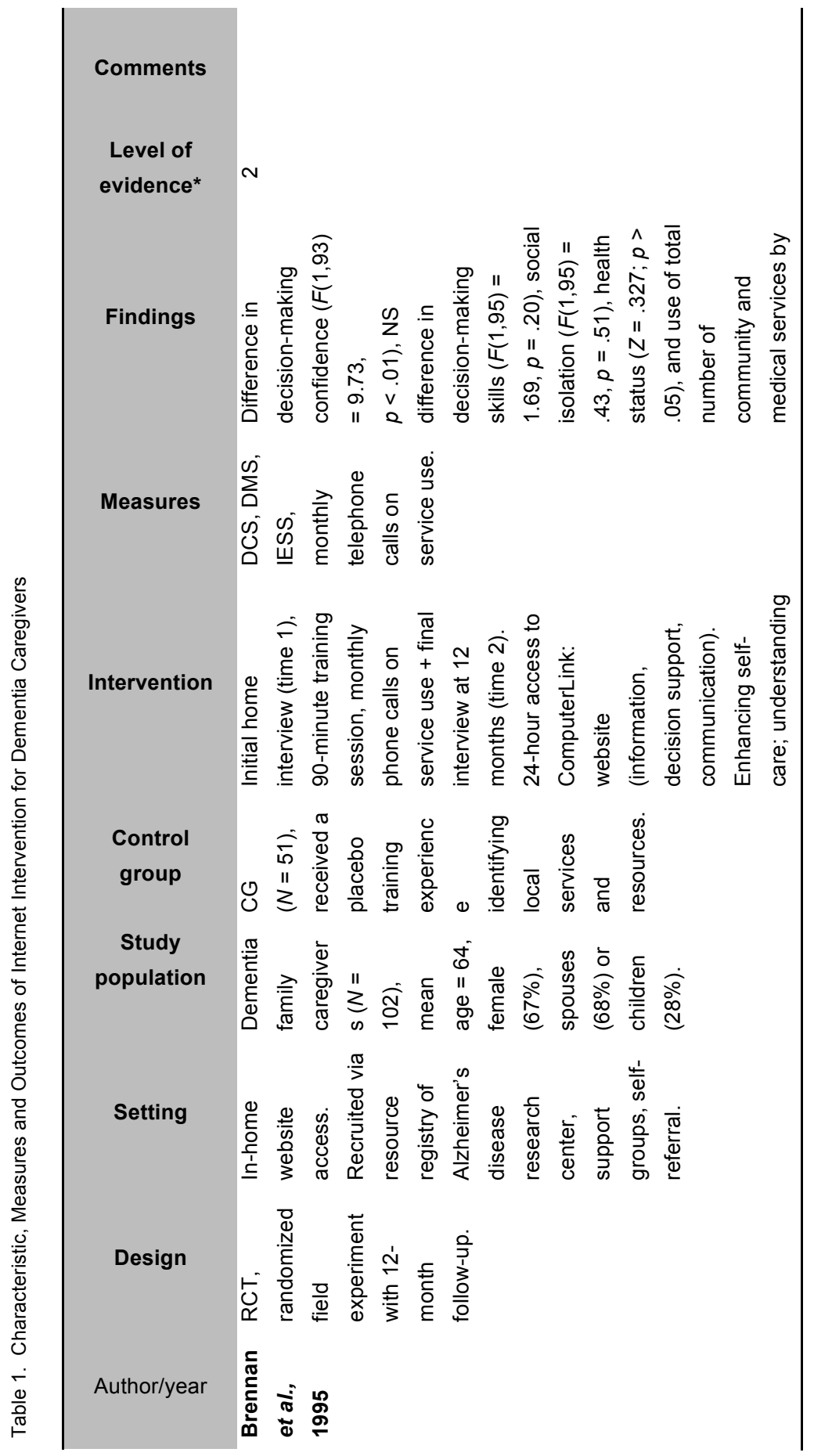




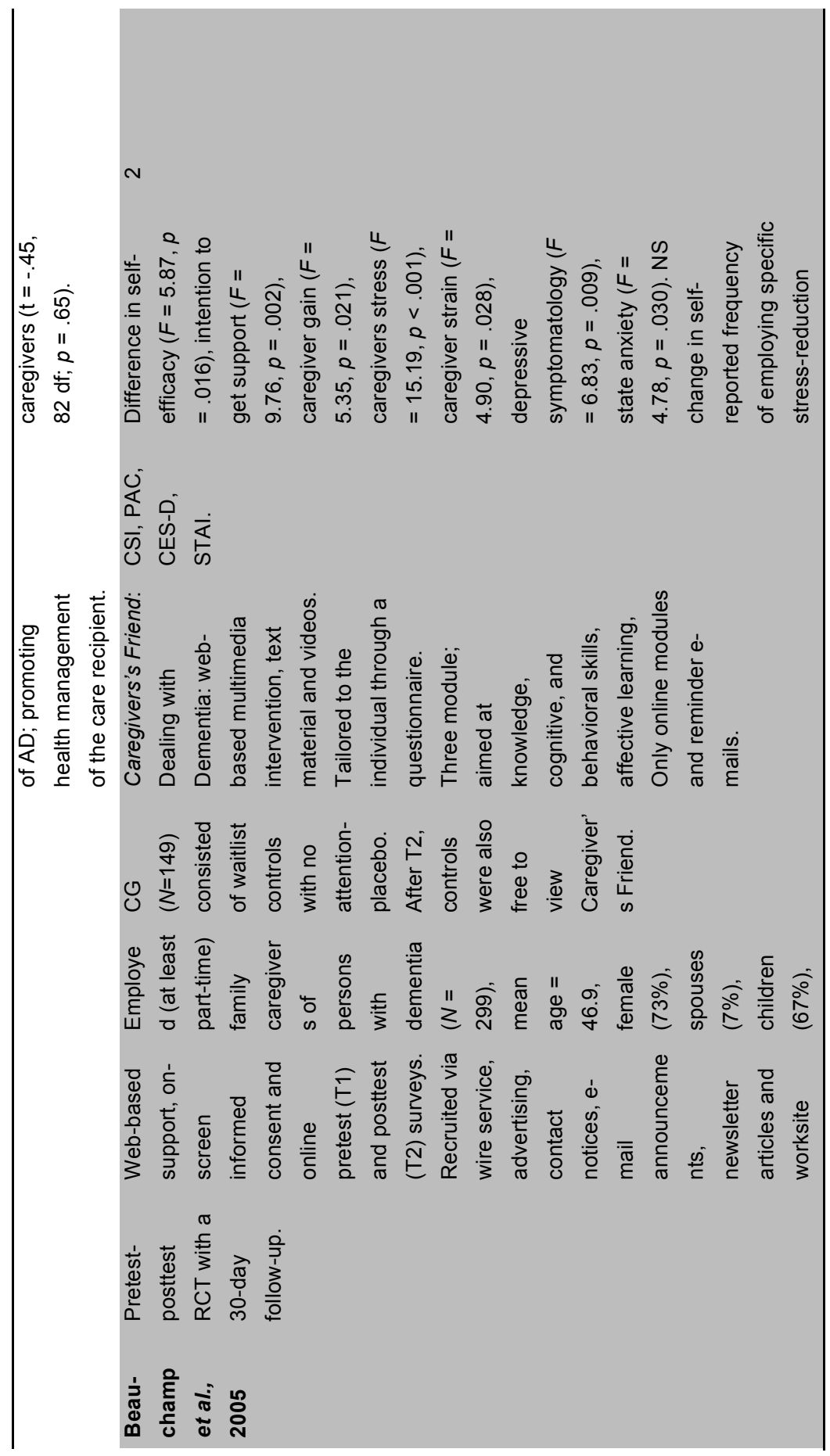




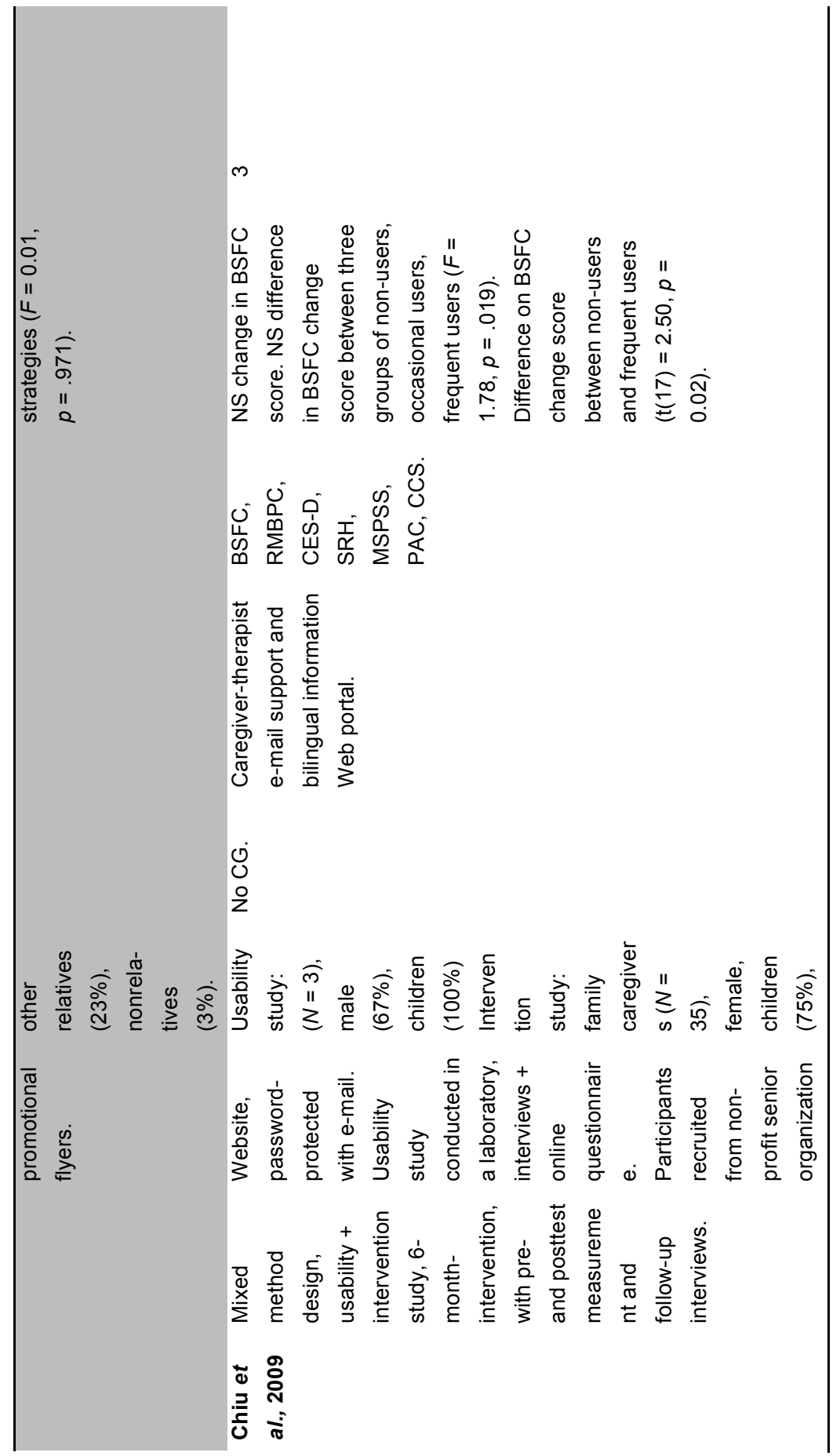




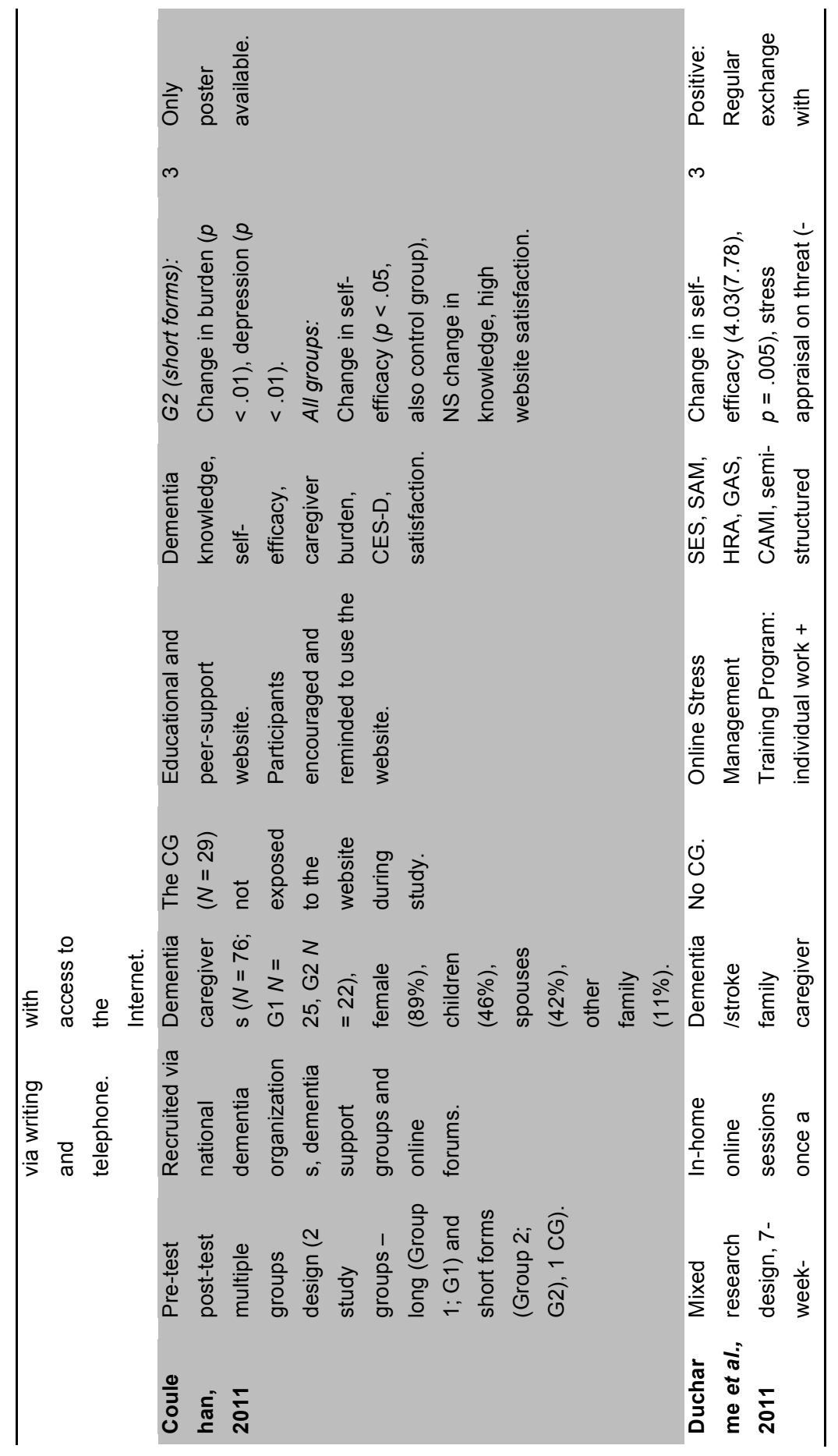




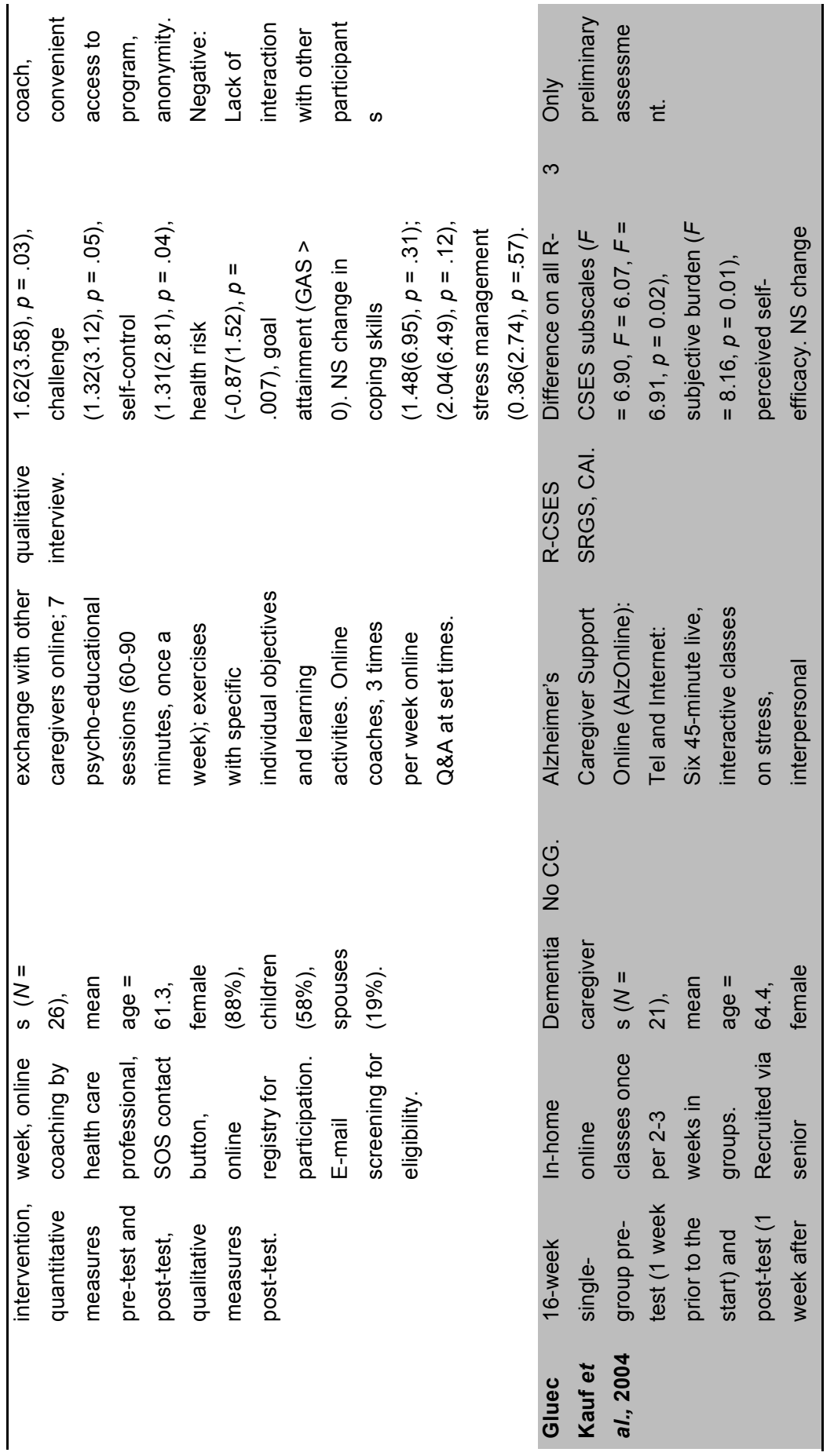




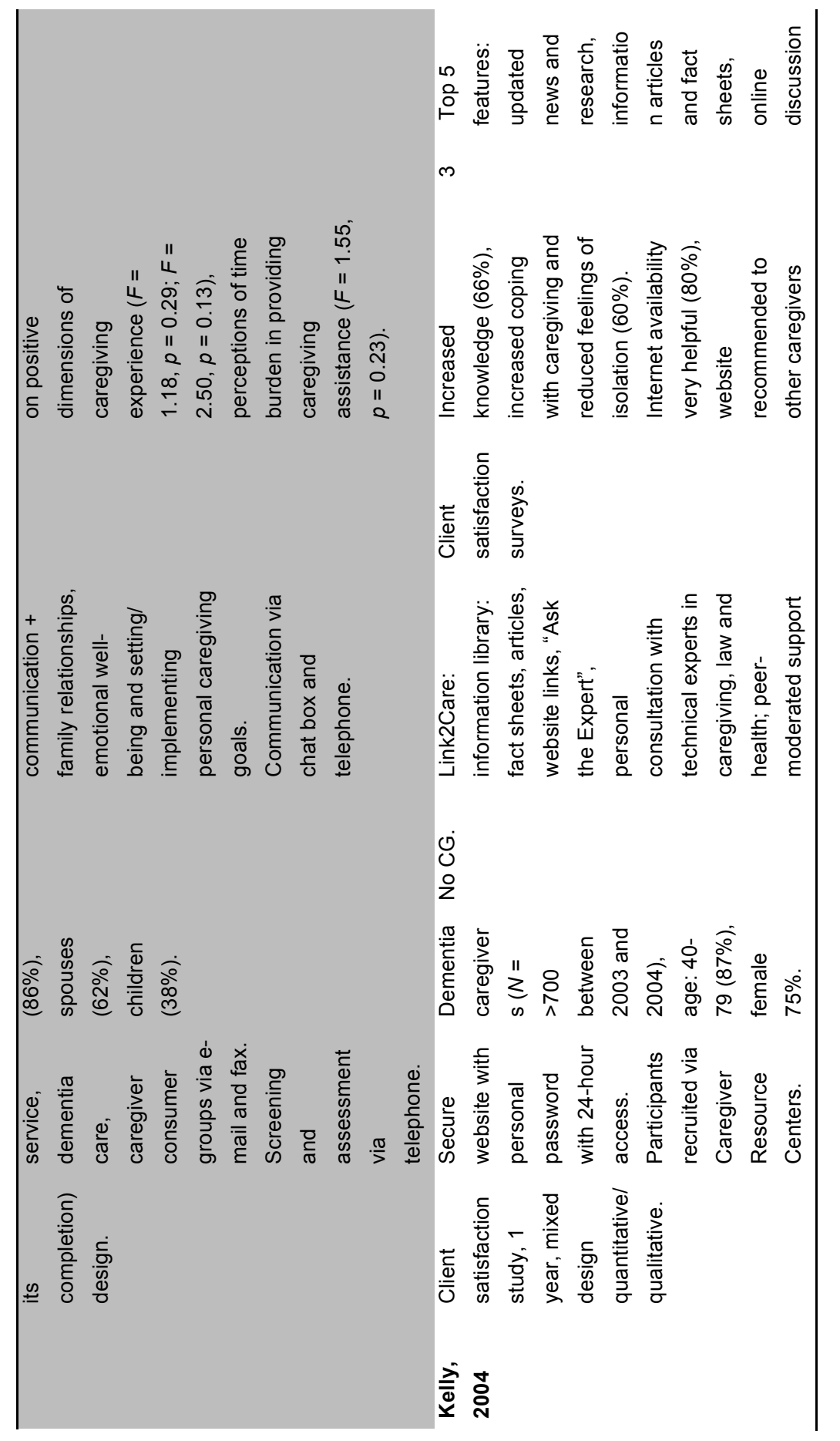




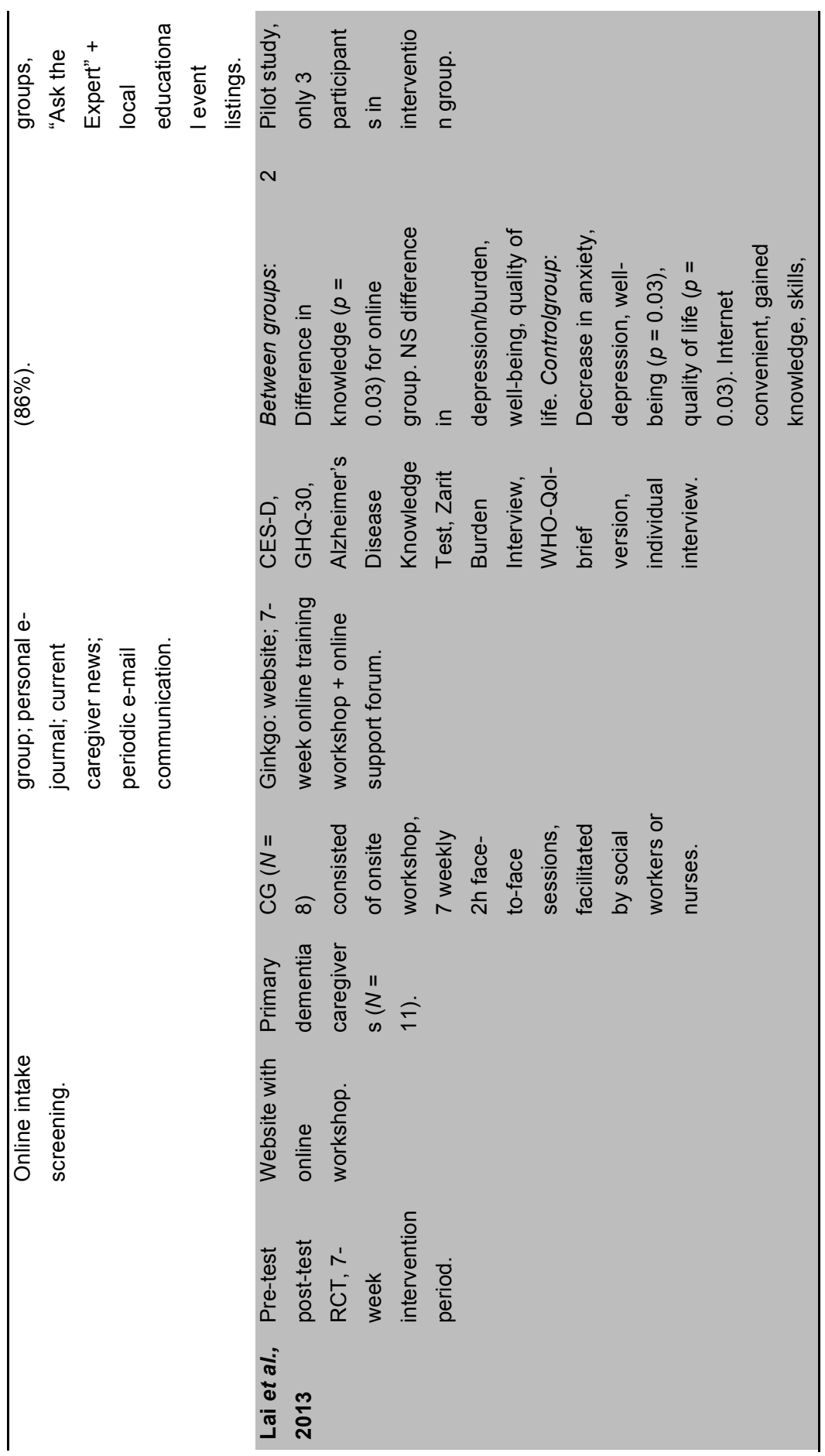




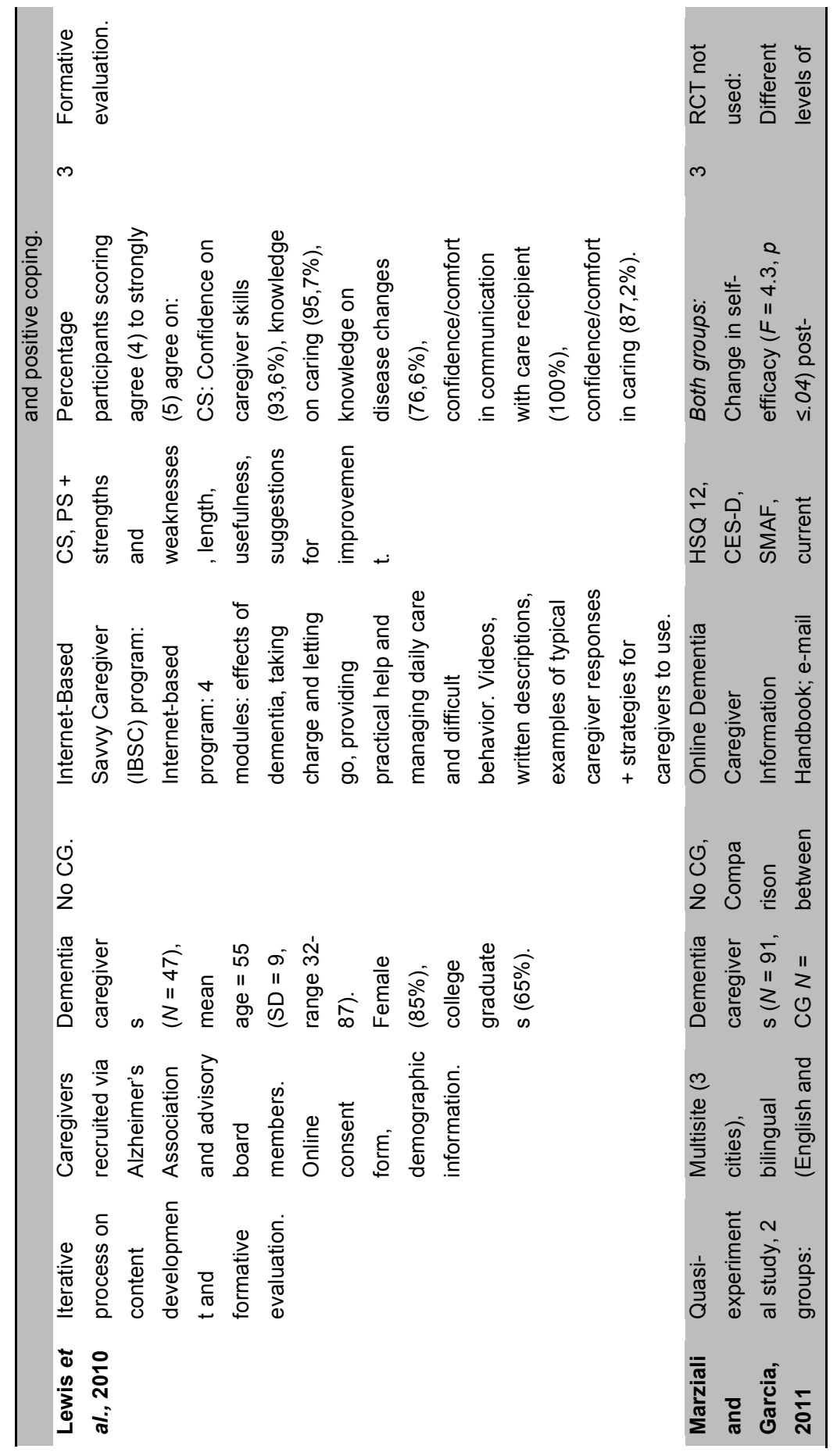




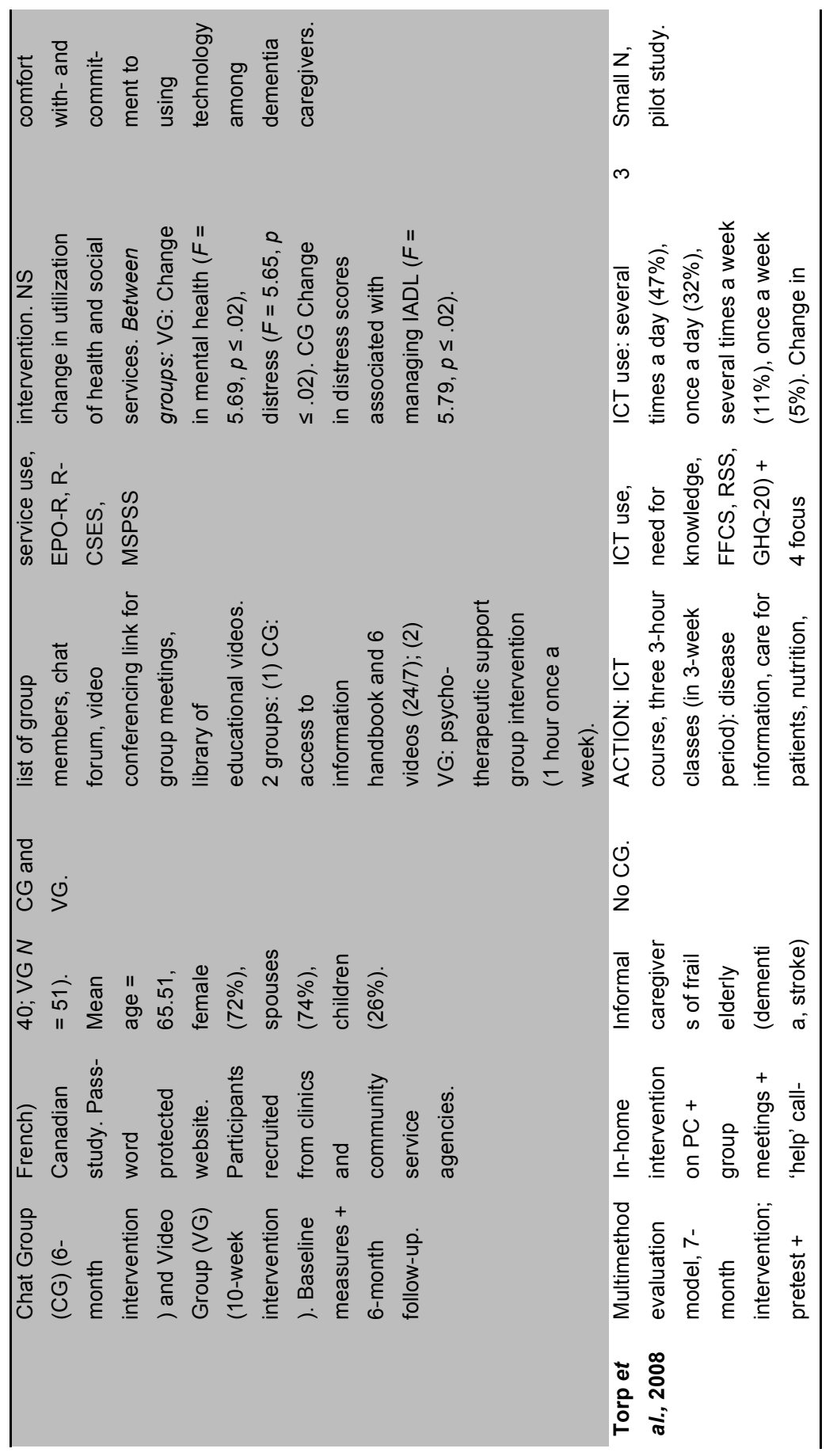




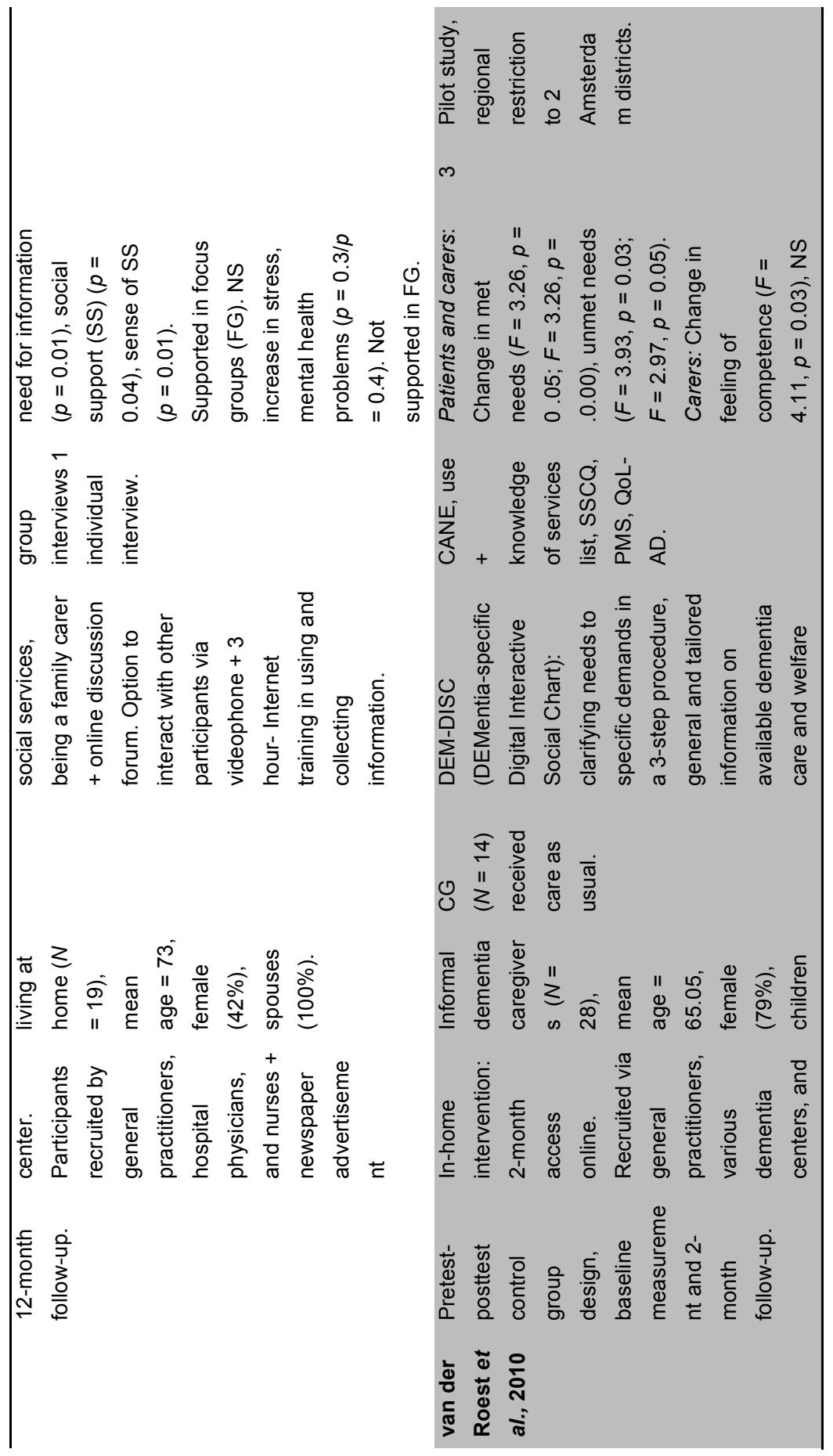




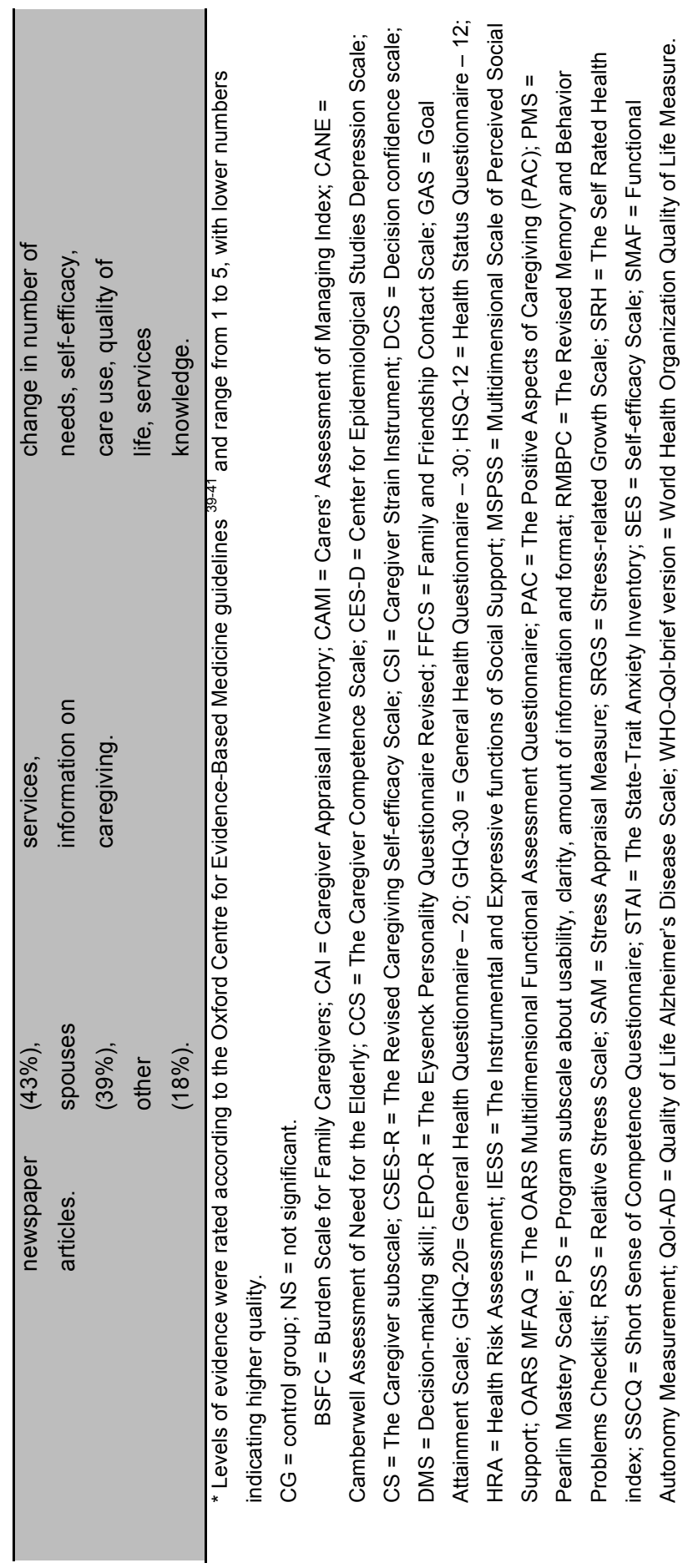




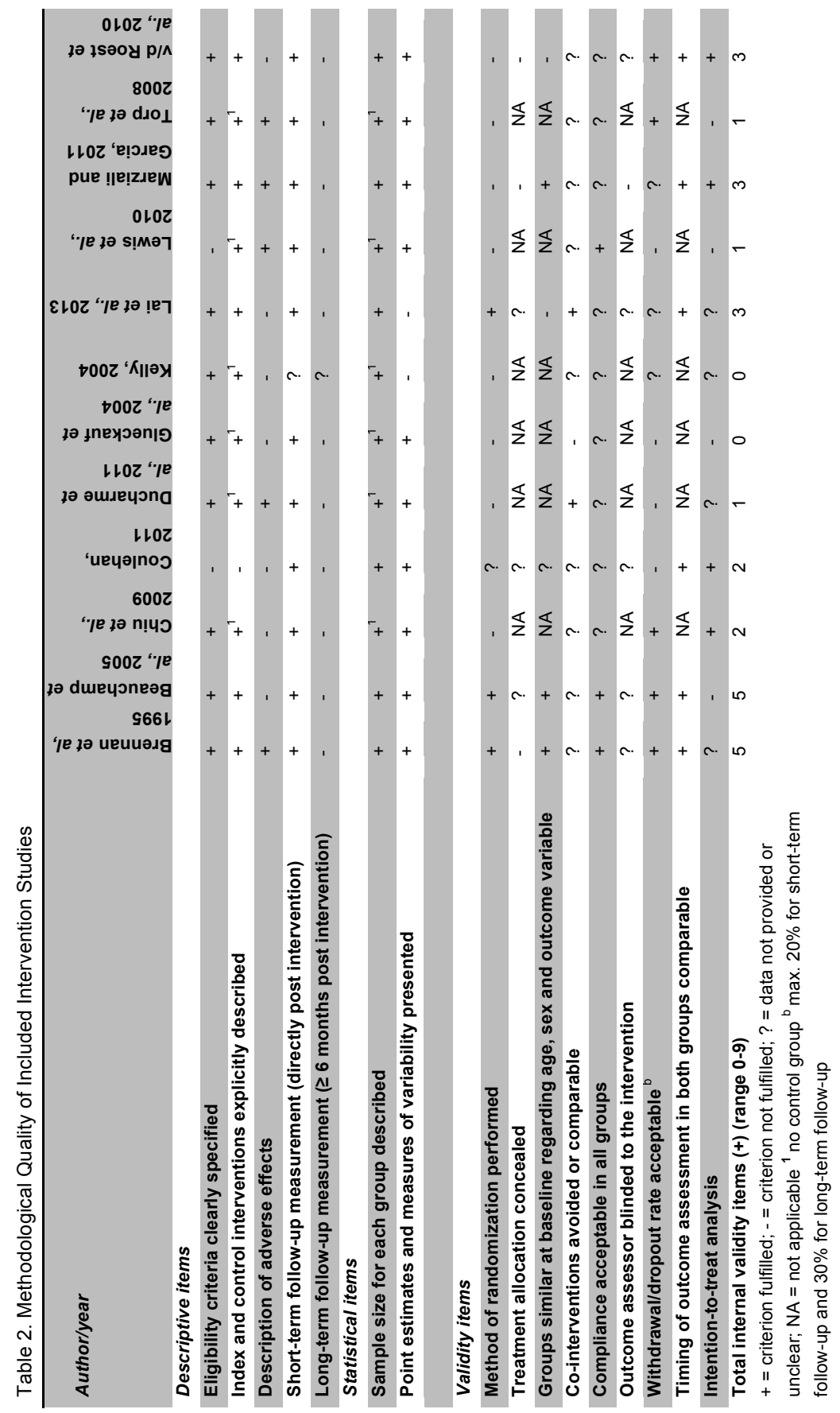




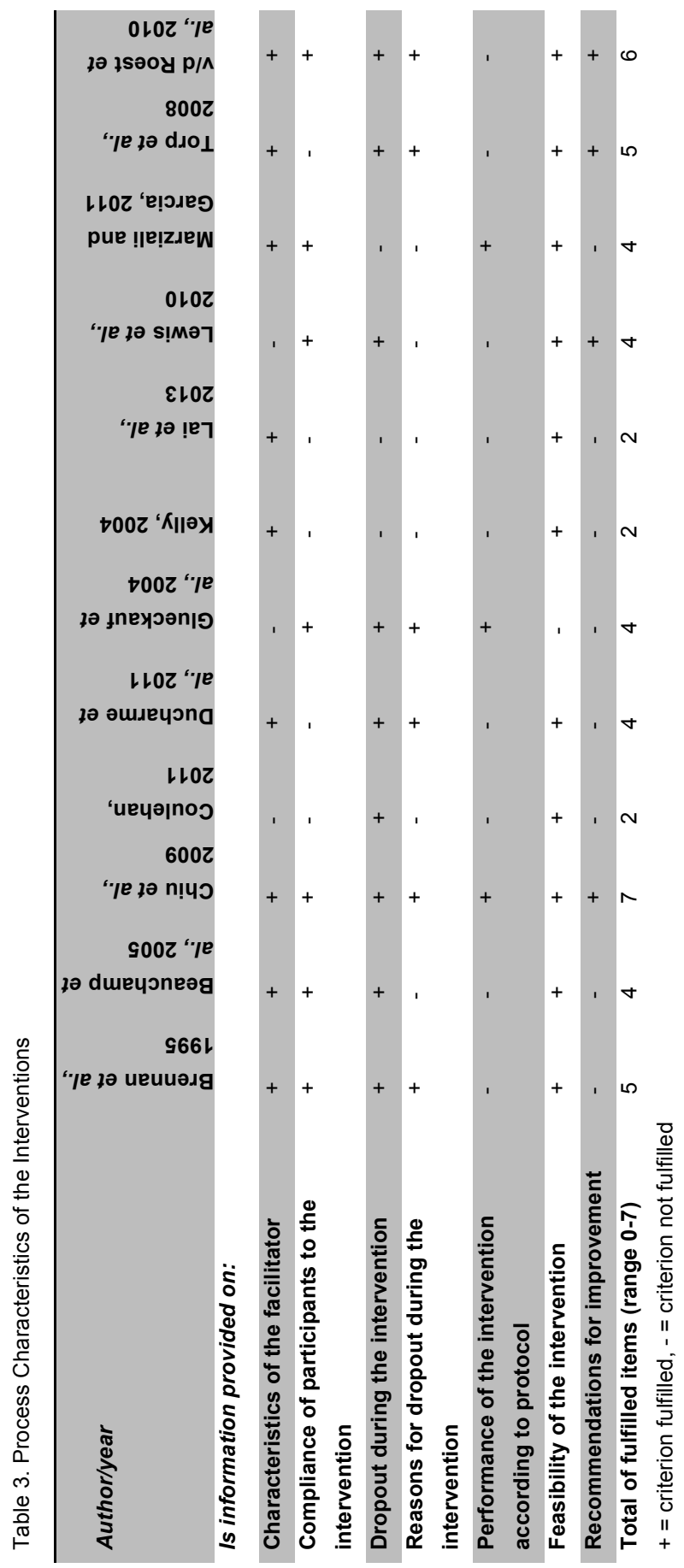


As shown in Table 2, the methodological quality of the included studies varied. The observed total internal validity score ranged from zero to five out of nine. Two of the 12 included trials fulfilled five criteria and were considered to be of good methodological quality. ${ }^{33,38}$ Five of the 12 studies included a control group, ${ }^{27,33-35,38}$ but only three used a random assignment procedure. ${ }^{27,33,38}$ Concealment of treatment allocation, avoided or comparable co-interventions, blinding of the outcome assessor and an intention-to-treat analysis were often not described, or were unclear. None of the selected studies included, or did not clearly specify, a long-term followup period. In contrast, all of the studies described the sample size, and most of the studies explicitly specified eligibility criteria, ${ }^{27-34,37,38}$ performed a short-term follow-up measurement, ${ }^{29-38}$ and presented point estimates and measures of variability. ${ }^{29-38}$

\section{Process characteristics}

Table 3 presents the reported process characteristics of the interventions. The observed total number of fulfilled items ranged from two to seven. Of the seven process items across the 12 trials, 58\% (49/84 items) were fulfilled. On average, nine of the included trials presented clear information on the facilitator conducting the intervention, ${ }^{27-34,38} 11$ on feasibility of the intervention, ${ }^{13,27-35,38}$ and seven on the compliance of the participants. ${ }^{29,31,33,34,36-38}$ Recommendations for improvement of the intervention were specified in four articles ${ }^{29,32,34,36}$ and information on the performance of the intervention according to protocol was less commonly reported; three papers ${ }^{29,31,37}$ provided this information.

\section{Outcomes}

\section{Quantitative outcomes}

Overall, (small) significant primary outcome effects of the intervention were observed in six studies. Specifically, improvement of the caregiver's well-being was observed in measures of depression, ${ }^{35,38}$ sense of competence, ${ }^{34}$ decision-making confidence, ${ }^{33}$ self-efficacy, ${ }^{30,31,37,38}$ and burden. ${ }^{35,37}$ Then again, caregiver burden did not decrease significantly in all studies. ${ }^{27,29}$ Chiu and colleagues ${ }^{29}$ did, however, observe significant differences in caregiver burden between non-users and frequent users of the program. Additionally, increased intention to seek support was reported, ${ }^{38}$ as well as caregiver gain ${ }^{38}$ and self-control, ${ }^{30}$ whereas caregiver stress and strain decreased. ${ }^{38}$ Furthermore, in one study, caregivers reported being able to achieve their previously set objectives. ${ }^{30}$ 
Lack of effect was also reported. For instance, no significant effects between groups or within the treatment group were found with respect to quality of life ${ }^{34}$ or coping skills. ${ }^{30}$ Additionally, the use of stress management techniques among caregivers $^{30}$ and social isolation and health status did not improve. ${ }^{33}$ In addition, one study reported improvements in the control group (onsite workshops) rather than the intervention group. In this control group, anxiety and depression decreased significantly and well-being and quality of life increased. People in the online intervention group did not improve with respect to these outcomes. ${ }^{27}$

\section{Qualitative outcomes}

Several studies reported carers' experiences as additional outcomes of their interventions, collected through individual- and focus group interviews postintervention. Increased knowledge of the disease and caring was described in some papers, ${ }^{27,28,36}$ in addition to increased coping with caregiving, ${ }^{27,28}$ confidence in caregiver skills $^{27,30,36}$ and reduced feelings of isolation. ${ }^{28}$

\section{DISCUSSION}

In this review, we aimed to provide a systematic overview of the effectiveness and feasibility of supportive Internet-based interventions for informal caregivers of patients with dementia. We identified 12 studies that investigated the effectiveness of different dementia caregiver-oriented interventions delivered through the Internet. The outcomes demonstrate that Internet interventions can result in positive effects on the well-being of informal dementia caregivers. In particular, multicomponent programs that combined information, tailored caregiving strategies and contact with other caregivers resulted in positive effects on confidence, self-efficacy, stress, burden and depression. ${ }^{30,31,35,37,38}$ Internet interventions that focused primarily on providing caregivers with information were less likely to improve caregiver wellbeing. ${ }^{33,34}$ These results are consistent with the conclusions of Gitlin and colleagues, ${ }^{42}$ emphasizing the need for tailored and multicomponent interventions. The results also demonstrate that guidance by a coach could be a noteworthy extension to an online intervention for informal dementia caregivers. ${ }^{30,37}$ The importance of a coach or therapist has also been demonstrated in Internet treatments for depression. ${ }^{43}$ Furthermore, caregivers themselves stressed the benefits of guidance by a coach; they stated that these exchanges increased their commitment to the intervention and boosted their confidence to implement learned strategies. ${ }^{30}$ 
Moreover, Lai and colleagues ${ }^{27}$ mentioned that the support function of online interactions between caregivers should not be overlooked. The latter is supported by the findings of Ducharme et al. ${ }^{30}$; caregivers who followed their program also mentioned a specific need for contact with other caregivers, specifically to break their isolation and to feel more 'normal'. Nonetheless, assessment of the process characteristics in the included studies indicates that performance according to protocol is often ignored or not reported, making it more difficult to interpret specific intervention effects and feasibility.

It should be noted that the methodological quality of the majority of the included studies was limited. Only five studies ${ }^{27,33-35,38}$ compared the results of the intervention with a control group, and most studies $(n=9)$ exhibited a LOE of three or lower, indicating a low quality of evidence. However, since we used two quality appraisals to rate and categorize the investigated studies, it is possible that some studies received too negative ratings. In addition, even though two reviewers rated methodological quality and process characteristics independently, we should remark that it was difficult to score all items for the included studies. For instance, compliance with the intervention was difficult to assess because most studies failed to mention compliance and because the studies that did report compliance failed to indicate when compliance was considered acceptable. It can be difficult to state what acceptable compliance is, as it is very hard to determine the acceptable intensity, duration or number and frequency of sessions for Internet interventions. Most studies did not state how often participants should visit the website, and one study ${ }^{38}$ identified favorable outcomes with an average of only 32 minutes of exposure to the program. A possible explanation is that participants in the treatment group may have gone on to visit other websites or seek resources outside the intervention, which also can be seen as a positive consequence of the program ${ }^{38}$. In addition, our results demonstrate that none of the included studies described or concealed treatment allocation. This might be because it is nearly impossible to blind the participants in psychosocial intervention trials with respect to the intervention to which they have been assigned. Overall, the studies with a higher evidence quality based on the LOE and the criteria list of the Cochrane Back Review Group ${ }^{33,38}$ supported the findings of the importance of multicomponent, tailored Internet interventions to improve caregiver functioning on confidence, self-efficacy or depression. Nevertheless, it is possible that only positive results were published, indicating a publication bias in the available evidence. More research with randomized controlled samples assessing 
interventions performed according to protocol is needed to make stronger statements about the effects of supportive Internet interventions and their most promising elements.

The current review is not without limitations. First, the included studies consisted of complex and inconsistent interventions and designs, making pooling of the data impossible. Therefore, it was more difficult to compare the outcomes of the studies. However, we did strengthen the consistency of the results of the present study through the use of a standardized data extraction form ${ }^{26}$. Second, as we wanted to achieve a comprehensive coverage of literature and as high-quality studies were scarce, we included many low-quality studies, resulting in the possibility of bias in a number of areas. For our conclusions, we focused mainly on studies of higher quality and, as mentioned, some studies may have received too negative ratings. Nevertheless, the results should be interpreted with caution.

Despite these limitations, we can conclude that Internet interventions to support dementia caregivers are a relatively new but promising extension to the currently offered care as usual. Caregivers of patients with chronic diseases, such as brain damage ${ }^{44}$ and heart diseases, ${ }^{45}$ already use supportive technology and report positive effects on well-being. At present, the growth of remote support for dementia caregivers is increasing, given the current development of new Internet interventions ${ }^{46,47}$ and telephone interventions. ${ }^{39-41,48}$ Although telephone interventions are qualitatively different, research on effects of telephone interventions concurs with the outcomes of the currently reviewed Internet-based studies. Combining tailored information with the help of a coach and the possibility to interact with other caregivers results in successful outcomes for dementia caregivers. ${ }^{39-41}$ These recent developments could provide a promising prospect for the digital generation of supportive interventions.

This review might cause healthcare professionals to consider online support for informal dementia caregivers in addition to the already provided care as usual. However, given the limited number of studies of high methodological quality, we can conclude that there is a need for further investigation in the field of Internet-based support for informal dementia caregivers in order to contribute to cost-effective care and improve the well-being of dementia caregivers around the globe, as well as lower the threshold of seeking help. 


\section{References}

1. Alzheimer Disease International. World Alzheimer Report. London: ADI, 2010.

2. Donaldson C, Burns A. Burden of Alzheimer's disease: helping the patient and caregiver. J Geriatr Psychiatry Neurol 1999; 12(1): 21-8.

3. Vitaliano PP, Zhang J, Scanlan JM. Is caregiving hazardous to one's physical health? A meta-analysis. Psychol Bull 2003; 129(6): 946-72.

4. de Vugt ME, Stevens F, Aalten P, et al. Do caregiver management strategies influence patient behaviour in dementia? Int $J$ Geriatr Psychiatry 2004; 19(1): 85-92.

5. Brodaty H, Donkin M. Family caregivers of people with dementia. Dialogues Clin Neurosci 2009; 11(2): 217-28.

6. Van Mierlo LD, Meiland FJ, Van der Roest HG, Droes RM. Personalised caregiver support: effectiveness of psychosocial interventions in subgroups of caregivers of people with dementia. Int J Geriatr Psychiatry 2012; 27(1): 1-14.

7. Selwood A, Johnston K, Katona C, Lyketsos C, Livingston G. Systematic review of the effect of psychological interventions on family caregivers of people with dementia. J Affect Disord 2007; 101(1-3): 75-89.

8. Olazaran J, Reisberg B, Clare L, et al. Nonpharmacological therapies in Alzheimer's disease: a systematic review of efficacy. Dement Geriatr Cogn Disord 2010; 30(2): 161-78.

9. Wimo A, Jonsson L, Gustavsson A, et al. The economic impact of dementia in Europe in 2008-cost estimates from the Eurocode project. Int $J$ Geriatr Psychiatry 2011; 26(8): 825-32.

10. Macdonald A, Cooper B. Long-term care and dementia services: an impending crisis. Age Ageing 2007; 36(1): 16-22.

11. Glueckauf RL, Loomis JS. Alzheimer's Caregiver Support Online: Lessons learned, initial findings and future directions. Neurorehabilitation 2003; 18(2): 135-46.

12. Martin-Carrasco M, Martin MF, Valero CP, et al. Effectiveness of a psychoeducational intervention program in the reduction of caregiver burden in Alzheimer's disease patients' caregivers. Int J Geriatr Psychiatry 2009; 24(5): 489-99.

13. Lewis BA, Williams DM, Neighbors CJ, Jakicic JM, Marcus BH. Cost Analysis of Internet vs. Print Interventions for Physical Activity Promotion. Psychol Sport Exerc 2010; 11(3): 246-9. 
14. Serafini JD, Damianakis T, Marziali E. Clinical practice standards and ethical issues applied to a virtual group intervention for spousal caregivers of people with Alzheimer's. Soc Work Health Care 2007; 44(3): 225-43.

15. Robinson L, Brittain K, Lindsay S, Jackson D, Olivier P. Keeping In Touch Everyday (KITE) project: developing assistive technologies with people with dementia and their carers to promote independence. Int Psychogeriatr 2009; 21(3): 494-502.

16. Topo P. Technology Studies to Meet the Needs of People With Dementia and Their Caregivers A Literature Review. Journal of Applied Gerontology 2009; 28(1): 5-37.

17. Thompson CA, Spilsbury K, Hall J, Birks Y, Barnes C, Adamson J. Systematic review of information and support interventions for caregivers of people with dementia. BMC Geriatr 2007; 7: 18.

18. Zickuhr K, Madden M, editors. Older Adults and Internet Use: Pew Internet \& American Life Project; 2012.

19. van Tulder MW, Assendelft WJ, Koes BW, Bouter LM. Method guidelines for systematic reviews in the Cochrane Collaboration Back Review Group for Spinal Disorders. Spine (Phila Pa 1976) 1997; 22(20): 2323-30.

20. van Tulder MW, Cherkin DC, Berman B, Lao L, Koes BW. The effectiveness of acupuncture in the management of acute and chronic low back pain. $A$ systematic review within the framework of the Cochrane Collaboration Back Review Group. Spine (Phila Pa 1976) 1999; 24(11): 1113-23.

21. OCEBM Levels of Evidence Working Group. The Oxford 2011 Levels of Evidence http://www.cebm.net/index.aspx?o=5653 Oxford Centre for Evidence-Based Medicine; 2011.

22. Livingston G, Johnston K, Katona C, Paton J, Lyketsos CG. Systematic review of psychological approaches to the management of neuropsychiatric symptoms of dementia. Am J Psychiatry 2005; 162(11): 1996-2021.

23. Visser K, Katchamart W, Loza E, et al. Multinational evidence-based recommendations for the use of methotrexate in rheumatic disorders with a focus on rheumatoid arthritis: integrating systematic literature research and expert opinion of a broad international panel of rheumatologists in the $3 \mathrm{E}$ Initiative. Ann Rheum Dis 2009; 68(7): 1086-93. 
24. Petrowsky H, Demartines N, Rousson V, Clavien PA. Evidence-based value of prophylactic drainage in gastrointestinal surgery: a systematic review and meta-analyses. Ann Surg 2004; 240(6): 1074-84; discussion 84-5.

25. Zijlstra GA, van Haastregt JC, van Rossum E, van Eijk JT, Yardley L, Kempen GI. Interventions to reduce fear of falling in community-living older people: a systematic review. J Am Geriatr Soc 2007; 55(4): 603-15.

26. The Cochrane Collaboration. Cochrane Handbook for Systematic Reviews of Interventions. In: Higgins JPT, Green S, editors.: Available from http://www.cochrane-handbook.org

27. Lai CKY, Wong LF, Liu KH, Lui W, Chan MF, Yap LSY. Online and onsite training for family caregivers of people with dementia: results from a pilot study. Int J Geriatr Psychiatry 2013; 28(1): 107-8.

28. Kelly K. Link2Care: Internet-Based Information and Support for Caregivers. Generations 2004; 27(4).

29. Chiu T, Marziali E, Colantonio A, et al. Internet-based caregiver support for Chinese Canadians taking care of a family member with alzheimer disease and related dementia. Can J Aging 2009; 28(4): 323-36.

30. Ducharme F, Dube V, Levesque L, Saulnier D, Giroux F. An Online Stress Management Training Program as a Supportive Nursing Intervention for Family Caregivers of an Elderly Person. canadian nursing informatics journal 2011; 6(2): 13-44.

31. Marziali E, Garcia LJ. Dementia caregivers' responses to 2 Internet-based intervention programs. Am J Alzheimers Dis Other Demen 2011; 26(1): 36-43.

32. Torp S, Hanson E, Hauge S, Ulstein I, Magnusson L. A pilot study of how information and communication technology may contribute to health promotion among elderly spousal carers in Norway. Health Soc Care Comm 2008; 16(1): 75-85.

33. Brennan PF, Moore SM, Smyth KA. The effects of a special computer network on caregivers of persons with Alzheimer's disease. Nurs Res 1995; 44(3): 166-72.

34. van der Roest HG, Meiland FJM, Jonker C, Droes RM. User evaluation of the DEMentia-specific Digital Interactive Social Chart (DEM-DISC). A pilot study among informal carers on its impact, user friendliness and, usefulness. Aging Ment Health 2010; 14(4): 461-70. 
35. Coulehan MB. Impact of a Web-Based Educational and Peer-Support Intervention for Dementia Caregivers. Aging in America Conference American Society on Aging 2011.

36. Lewis ML, Hobday JV, Hepburn KW. Internet-based program for dementia caregivers. Am J Alzheimers Dis Other Demen 2010; 25(8): 674-9.

37. Glueckauf RL, Ketterson TU, Loomis JS, Dages P. Online support and education for dementia caregivers: overview, utilization, and initial program evaluation. Telemed J E Health 2004; 10(2): 223-32.

38. Beauchamp N, Irvine AB, Seeley J, Johnson B. Worksite-based internet multimedia program for family caregivers of persons with dementia. Gerontologist 2005; 45(6): 793-801.

39. Wilz G, Schinkothe D, Soellner R. Goal attainment and treatment compliance in a cognitive-behavioral telephone intervention for family caregivers of persons with dementia. GeroPsych 2011; 24(3): 115-25.

40. McHugh JE, Wherton JP, Prendergast DK, Lawlor BA. Teleconferencing as a source of social support for older spousal caregivers: initial explorations and recommendations for future research. Am J Alzheimers Dis Other Demen 2012; 27(6): 381-7.

41. Forducey PG, Glueckauf RL, Bergquist TF, Maheu MM, Yutsis M. Telehealth for persons with severe functional disabilities and their caregivers: facilitating self-care management in the home setting. Psychol Serv 2012; 9(2): 144-62.

42. Gitlin LN, Belle SH, Burgio LD, et al. Effect of multicomponent interventions on caregiver burden and depression: the REACH multisite initiative at 6-month follow-up. Psychol Aging 2003; 18(3): 361-74.

43. Andersson G, Cuijpers P. Internet-based and other computerized psychological treatments for adult depression: a meta-analysis. Cognitive Behaviour Therapy 2009; 38: 196-205.

44. Sander AM, Clark AN, Atchison TB, Rueda M. A web-based videoconferencing approach to training caregivers in rural areas to compensate for problems related to traumatic brain injury. $J$ Head Trauma Rehabil 2009; 24(4): 248-61.

45. Dew MA, Goycoolea JM, Harris RC, et al. An internet-based intervention to improve psychosocial outcomes in heart transplant recipients and family caregivers: development and evaluation. J Heart Lung Transplant 2004; 23(6): 745-58. 
46. Hayden LJ, Glynn SM, Hahn TJ, Randall F, Randolph E. The Use of Internet Technology for Psychoeducation and Support With Dementia Caregivers. Psychol Serv 2012; 9(2): 215-8.

47. Blom M, Bosmans JE, Cuijpers P, Zarit SH, Pot AM. Effectiveness and costeffectiveness of an Internet intervention for family caregivers of people with dementia: design of a randomized controlled trial. BMC Psychiatry 2013; 13(17).

48. van Mierlo LD, Meiland FJ, Droes RM. Dementelcoach: effect of telephone coaching on carers of community-dwelling people with dementia. Int Psychogeriatr 2012; 24(2): 212-22. 




\section{CHAPTER 3}

A mismatch between supply and demand of social support in dementia: a qualitative study on the perspectives of spousal caregivers and their social network members

Submitted

Alieske E.H. Dam, Lizzy M.M. Boots, Martin P.J. van Boxtel, Frans R.J. Verhey \& Marjolein E. de Vugt 


\section{ABSTRACT}

Objectives. This qualitative study integrates the dyadic data from spousal caregivers and their social network members to investigate reciprocal perspectives on barriers and facilitators to supply and demand of social support.

Methods. Ten spouses completed an ecogram, a social network card and an interview. The ecogram aimed to trigger subjective experiences regarding social support. 17 network members were interviewed. The qualitative analyses identified codes, categories and themes.

Results. Using an ecogram before the interview effectively triggered subjective experiences regarding social support. Four major themes emerged: (1) partners' needs and demand for support, which are divided into subthemes: (a) barriers to ask for support and (b) facilitators to ask for support; (2) network supply of support, which is divided into subthemes: (a) barriers to offer support and (b) facilitators to offer support; (3) a mismatch between supply and demand; and (4) openness in communication to repair the imbalance.

Discussion. Integrating social network perspectives was highly effective. A model identifying a mismatch between the supply and demand of social support was proposed, strengthened by a cognitive bias: caregivers reported to think for other social network members and vice versa. Openness in communication in formal and informal care systems might repair this mismatch. 


\section{INTRODUCTION}

Dementia has a major impact on the lives of family caregivers and social network members, especially on the persons providing primary care to the people with dementia (PwD) living in the community. The caregivers are faced with physical, emotional and economical challenges during the different phases of the disease. Consequently, they are prone to depression, social isolation and even physical complaints. $^{1}$

Clearly, social support for informal caregivers is essential for improving their health and quality of life. Research has demonstrated that social support and the sharing of stressful experiences contribute to improved caregiver well-being ${ }^{2,3}$ and buffers against experienced stressors. In contrast, lower levels of informal social support are associated with increased caregiver burden and feelings of depression. ${ }^{4,5}$ Nevertheless, primary caregivers of PwD often find it difficult to initiate requests for assistance and support from their own social network, thus increasing their risk for social isolation and burden. Although the non-use of formal services is often caused by the PwD's refusal, a lack of awareness on the part of the caregiver, or no perceived need for such services, ${ }^{6,7}$ only few studies have been conducted regarding the factors involved in the acceptance of informal support. Research has indicated that caregivers do not always accept offers of help because of the associated stigma ${ }^{8}$ or fear of the PwD's reactions. ${ }^{9}$

To lower the high caregiver threshold to seek support and to improve the current caregiver interventions, we need to examine the factors contributing to the acceptance of informal support. In this qualitative study, we explored the barriers and facilitators for both supply and demand of informal support in the context of the caregiver's social network. To date, few qualitative studies have incorporated the perspectives of both the spousal caregivers and their social network members. This assessment is important because informal caregivers must provide longer care in the community ${ }^{10,11}$ and increasingly must rely on the assistance and support from their social network.

In sum, we aimed to (1) explore spousal caregivers' needs, perceptions and experiences regarding informal support; (2) gain insight into the social network members' needs, perceptions and experiences regarding informal support towards spousal caregivers; and (3) identify the barriers and facilitators to the supply and demand of informal support within the informal care network. 


\section{METHODS}

\section{Participants}

Ten spousal caregivers of PwD and two members of their social network $(n=17)$ were recruited by the memory clinic of Maastricht University Medical Centre (MUMC+). Two network members were included to gain insight into the perspectives of both near and, when available, more distant network members. A selection of 10 participants was considered to be sufficient to obtain data saturation. ${ }^{12}$ All of the recruited spousal caregivers and $\mathrm{PWD}$ shared a household. There were no restrictions for the duration and type of dementia diagnosis. The clinician invited the spousal caregivers for participation in the study while visiting the memory clinic, and/or the researcher called them directly. Subsequently, they received a letter regarding their participation. In case the spousal caregiver provided permission, two members from his or her social network were approached and invited by a letter to participate in an interview. It was not possible to recruit the network members of two spousal caregivers. They either could not think of a significant other or the network members themselves did not accept the invitation because of their busy schedule or unwillingness to participate in the research. One spouse provided three network members for inclusion. The ethical committee of the faculty of Psychology and Neuroscience of Maastricht University approved this study (ECP-150 22-03-2015).

\section{Data collection}

First, written informed consent was obtained. Second, 1- to 2-hour semi-structured interviews were conducted. Depending on the participant's preference, the interviews were conducted either at the MUMC+ or in the participant's home. The interviews were structured according to a topic list based on the extant literature and experience from clinical practice. At the commencement of the interview with the spousal caregivers, an ecogram and social network card based on the Maastricht Social Network Analysis ${ }^{13}$ were completed. On the ecogram, the spousal caregivers named the people who were (1) very closely involved/or essential in providing support to the spousal caregiver, (2) somewhat less closely involved in providing support to the spousal caregiver on a regular basis, and (3) acquaintances who provided support only sporadically. Figure 1 shows the average ecogram size. Subsequently, a social network card was completed describing the characteristics of the persons listed on the ecogram, including the relationship to the spousal caregiver, age, geographical distance, type of support, frequency of support (face-to-face, telephone and e-mail) 
and the importance of that person using a visual analogue scale (VAS) ranging from 0 (not important) to 100 (extremely important). The ecogram and network card were not only completed to map the social network but more specifically to probe the caregivers' thoughts and feelings regarding the availability of the support persons and experienced informal support. Using sensitization techniques is a proven method to elicit perspectives and experiences on a topic from past memories. ${ }^{14,15}$ We applied the ecogram as an innovative tool to sensitize and capture personal support experiences. The ecogram served as a guide throughout the interview and was referred to, in the second part, in the request for open-ended questions. The topics in the interviews with both the spousal caregiver and their network members included types of support received or provided, barriers and facilitators associated with spousal caregiver needs for social support, or social network member's supply of support. When necessary, the participants were stimulated to elaborate or clarify some thoughts and feelings.

Additionally, data were collected on age, gender, education, total time spent on caring, satisfaction with the social network and burden scored using a VAS scale that ranged from 0 (no burden at all/not satisfied) to 100 (the highest imagined burden/very satisfied).

\section{Data analysis}

We performed a qualitative analysis to identify the codes, categories and themes in our data. ${ }^{12}$ The interviews were transcribed verbatim and scored independently by two authors (AD and LB), using Atlas.ti 1 for a Mac computer (Scientific Software Development $\mathrm{GmbH}$, Berlin). Both researchers used an inductive content analysis in which the codes and categories were derived from the data employing constant comparison and inductive reasoning. First, open codes were created covering all text fragments. Second, overlapping codes referring to a similar phenomenon were grouped into categories. Subsequently, higher-order themes were identified. ${ }^{12}$ After coding the 27 interviews, saturation occurred because no new codes emerged from the data. 


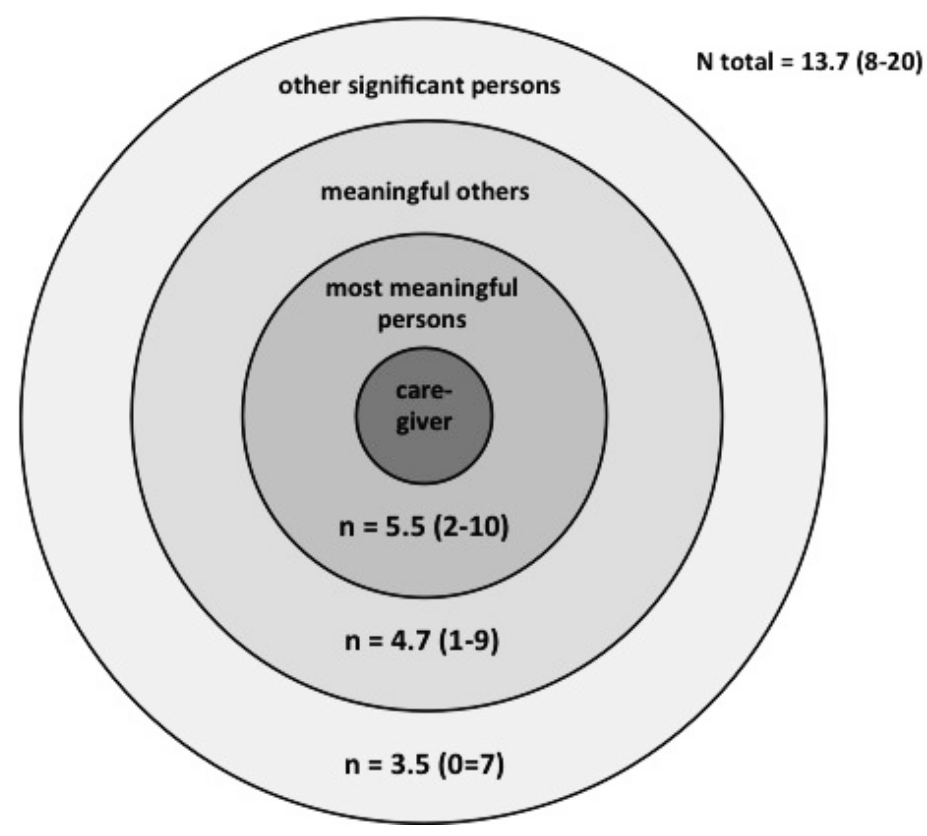

Figure 1. The ecogram that was completed during the interview with the spousal caregiver.

$\mathrm{n}=$ the mean number of contacts in every circle (i.e., with either individuals, couples or leisure clubs), followed by the range.

The categories and interpretations from both independent analyses were discussed in a meeting with the last author (MdV) to reach consensus regarding the categories, themes and emerging relationships.

\section{RESULTS}

\section{Participants}

The characteristics of the 10 spousal caregivers (mean age $=73.7$ years) and their 17 network members (mean age $=58.4$ years) are depicted in Tables 1 and 2 . The majority of the spouses $(70 \%)$ and network members $(82.4 \%)$ were female. The care recipients' diagnosis included Alzheimer's dementia $(n=6)$ or other dementias $(n=$ 4); the duration of time from the diagnosis ranged from 3 months to 5 years. 
Table 1. Background characteristics of the spousal caregivers $(n=10)$

and care recipients $(n=10)$

\begin{tabular}{ll}
\hline & Total Group \\
\hline Gender caregiver female, $\mathrm{n}(\%)$ & $7(70)$ \\
Age caregiver, mean (range) & $73.7(63-82)$ \\
Education level caregiver, mean (range) & $4.5(1-8)$ \\
Living together with care recipient (\%) & $10(100)$ \\
Years of caring (range) & $4(1-9)$ \\
Hours of caring per week, mean (range) & $37.6(10-98)$ \\
Subjective burden, mean (range) & $53.5(20-95)$ \\
Satisfaction with social network, mean (range) & $78.5(50-100)$ \\
& \\
Gender care recipient female (\%) & $3(30)$ \\
Age care recipient, mean (range) & $77.0(64-83)$ \\
Care recipient dementia diagnosis (\%) & \\
$\quad$ Alzheimer (\%) & $6(60)$ \\
Vascular (\%) & $3(30)$ \\
$\quad$ Mixed (AD/vascular) & $1(10)$ \\
& $3.03(0.30-5)$ \\
\hline Years since care recipient's diagnosis (range) & \\
\hline a Educational level ranges from 1 (unfinished primary education) to 8 (university degree), a mean of 4.5 \\
corresponds to an average education level. \\
${ }^{\mathrm{b}}$ Mean score on a visual analogue scale (VAS), ranging from 0 to 100.
\end{tabular}

Table 2. Background characteristics of the interviewed network members $(n=17)$

\begin{tabular}{ll}
\hline Gender female (\%) & Total Group \\
Age, mean (range) & $14(82.4)$ \\
Education level, caregiver, mean (SD) & $58.5(36-75)$ \\
Relationship to spousal caregiver (\%) & $4.5(2-8)$ \\
Friends & $3(17.7)$ \\
Neighbors & $2(11.8)$ \\
Others & $2(11.8)$ \\
Son & $3(17.7)$ \\
Daughter & $2(11.8)$ \\
Daughter-in-law & $1(5.9)$ \\
Sister & $3(17.7)$ \\
Niece & $1(5.9)$ \\
Years of caring (range) & $4.9(0-25)$ \\
Mean \# hours of caring per week (range) & $2.9(0-12)$ \\
Mean subjective burden (SD/range) & $24.7(0-80)$ \\
\hline${ }^{\mathrm{a}}$ education scores range from 1 (unfinished primary education) to 8 (university degree), a mean of 4.5 \\
corresponds to an average education level. \\
${ }^{\mathrm{b}=}$ Mean score on a visual analogue scale (VAS), ranging from 0 to 100.
\end{tabular}




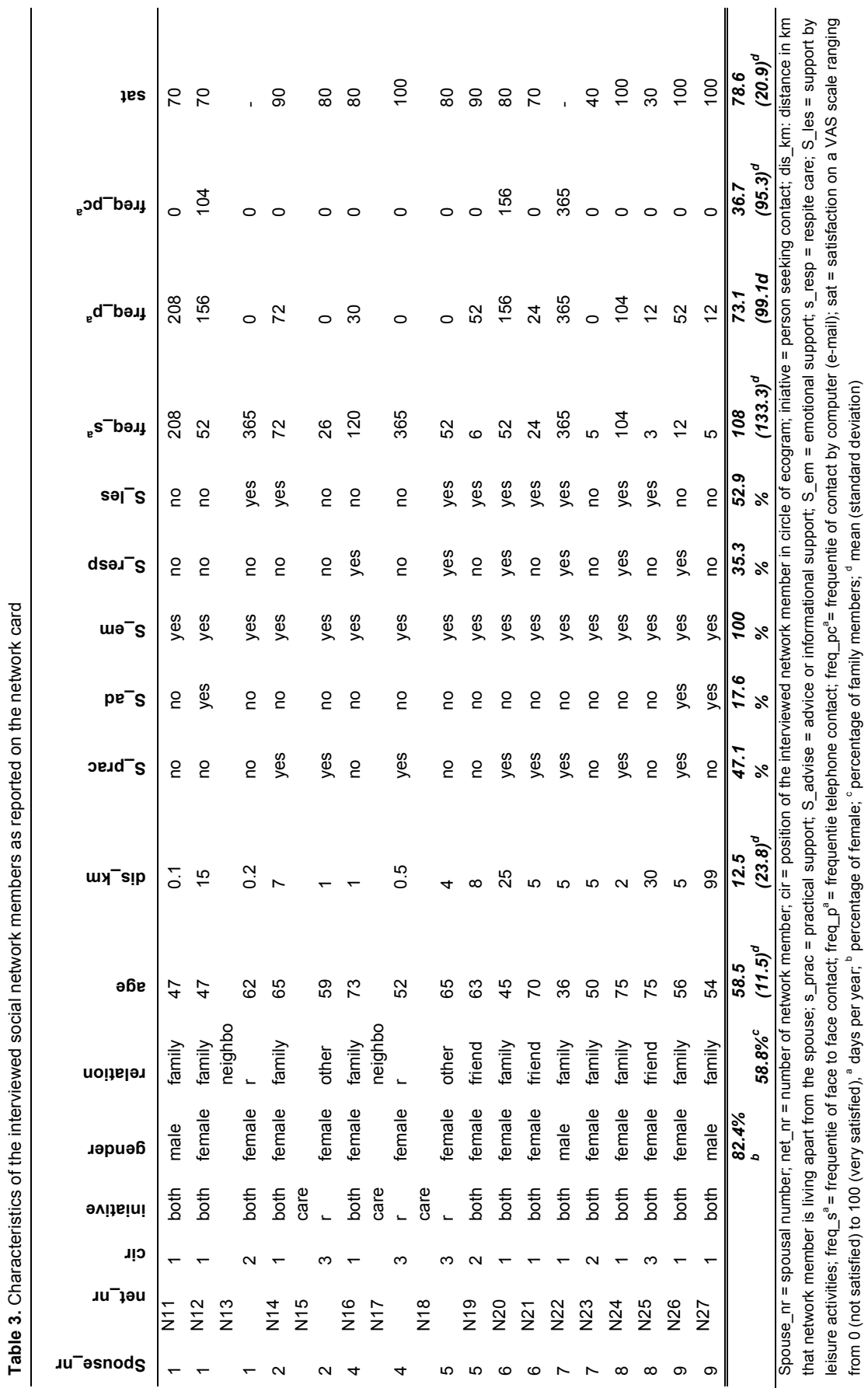


Table 3 shows the network members' characteristics (e.g., frequency of contact, type of support), as reported on the network card. The spouses were satisfied with their social network (VAS $=78.5$ ) and reported a moderate level of burden (VAS $=53.5$ ), which was indicated on a VAS (i.e., 0 , not satisfied/ no burden vs. 100 , very satisfied/high burden).

\section{Interviews with spousal caregivers and their social network members}

Four major themes were identified: (1) partners' needs and demands for support, which were divided into two subthemes: (a) barriers to ask for support, and (b) facilitators to ask for support; (2) network supply of support, which were divided into two subthemes: (a) barriers to offer support, and (b) facilitators to offer support; (3) a mismatch in the supply and demand of support; and (4) openness in communication to repair the imbalance.

An overview of themes and categories is provided in Table 4 and Figure 2, and they are clarified by the quotations in the following sections. Each quotation is marked by a code specifying the participant (e.g., $\mathrm{P}=$ partner vs. $\mathrm{N}=$ network member).

\section{Partners' needs and demand for social support}

The spousal caregivers clearly experience a need for social support. Respite care and practical support enable the spousal caregivers to continue their social activities and lead a normal life in addition to their caregiver role:

"I am convinced it is important to be able to escape from the situation. For example, by going to a music rehearsal. It is important for caregivers to be able to have different experiences. However, then you need to be able to flip a switch. I experience that when I close the door. It is not that I don't worry anymore, but I arranged something with the people around me, I can rely on them when something happens. As a caregiver, you need this; imagine how it would be if you have a small circle of people around you" (points to the ecogram, Fig. 1). $(\mathrm{P}, 1)$

Social support is not only considered to be practical, it also contributes to positive emotional caregiver experiences. Maintaining a social network can preserve feelings of usefulness, worthiness and belongingness:

"I take care of my contacts, I don't want to be only the partner of 


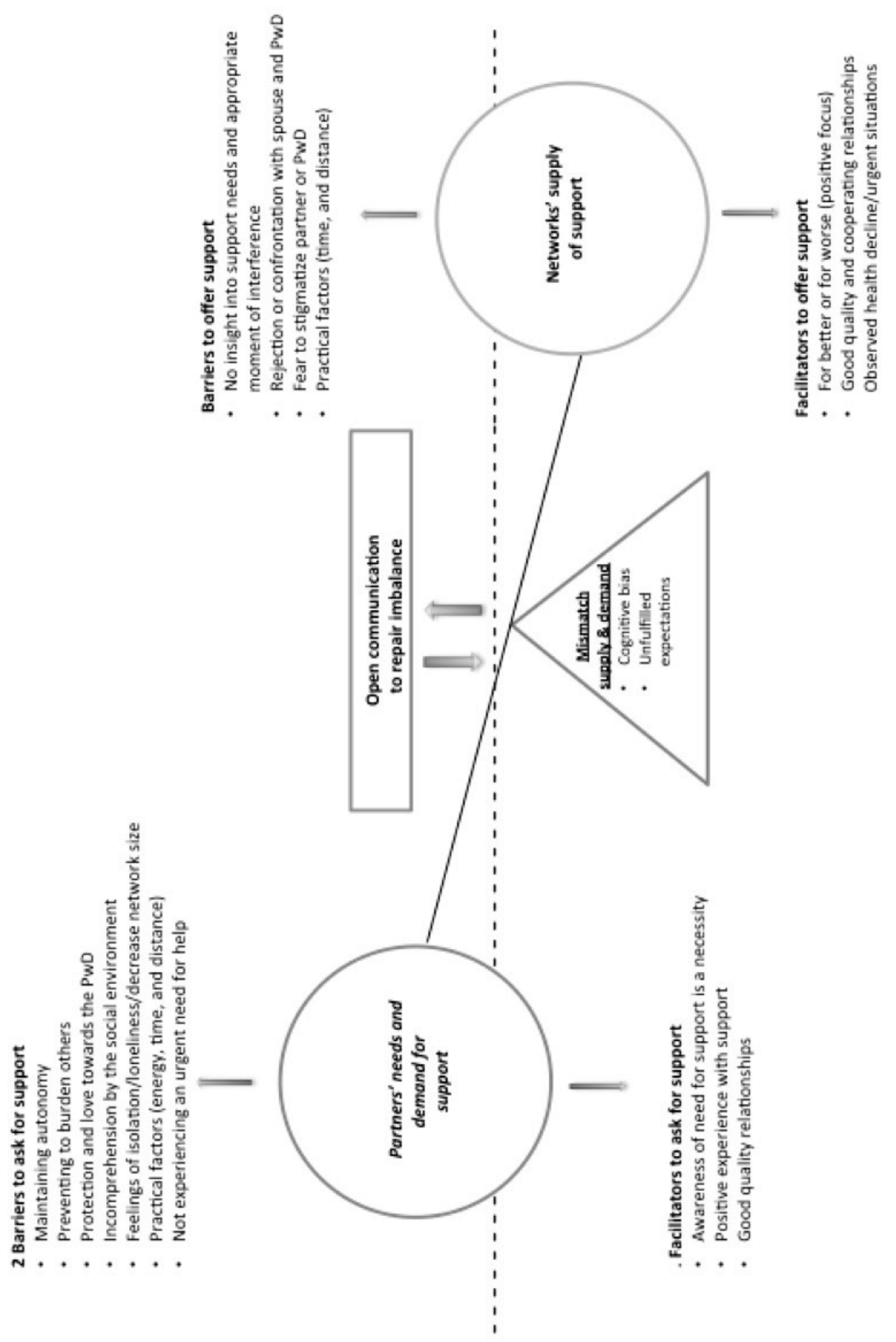

Figure 2. The proposed model of the mismatch between demand and supply of social support 
Table 4. Overview of categories and major themes

\begin{tabular}{|c|c|}
\hline Themes & Categories \\
\hline \multirow[t]{3}{*}{ Partners' needs and demands for support } & $\begin{array}{l}\text { Types of support (emotional, practical, } \\
\text { company, and respite) }\end{array}$ \\
\hline & Social participation and connectedness \\
\hline & Distraction/re-charging personal batteries \\
\hline \multirow[t]{7}{*}{ a) Barriers to ask for support } & Maintaining autonomy \\
\hline & Preventing becoming a burden to others \\
\hline & Protection and love towards the PwD \\
\hline & Incomprehension by the social environment \\
\hline & Feelings of isolation/loneliness \\
\hline & Practical factors (energy, time, and distance) \\
\hline & Not experiencing an urgent need for help \\
\hline \multirow[t]{3}{*}{ b) Facilitating factors to ask for support } & Awareness of need for support is a necessity \\
\hline & Positive experience with support \\
\hline & Good quality relationships \\
\hline \multirow[t]{2}{*}{ Networks' supply of support } & Willingness to help \\
\hline & $\begin{array}{l}\text { Being meaningful and enjoying and enriched } \\
\text { relationship }\end{array}$ \\
\hline \multirow[t]{4}{*}{ a) Barriers to offer support } & $\begin{array}{l}\text { No insight into support needs and at which } \\
\text { moment }\end{array}$ \\
\hline & $\begin{array}{l}\text { Rejection or confrontation with the spouse } \\
\text { and the PwD }\end{array}$ \\
\hline & Fear to stigmatize partner or PwD \\
\hline & Practical factors (time, and distance) \\
\hline \multirow[t]{3}{*}{ b) Facilitating factors to offer support } & \\
\hline & $\begin{array}{l}\text { Support for better or for worse (positive } \\
\text { focus) }\end{array}$ \\
\hline & $\begin{array}{l}\text { Good quality and cooperating relationships } \\
\text { within the network }\end{array}$ \\
\hline Mismatch between supply and demand & Observed decline in health and mood \\
\hline \multirow[t]{5}{*}{ Repairing the imbalance } & $\begin{array}{l}\text { Cognitive bias regarding each other's } \\
\text { intentions }\end{array}$ \\
\hline & $\begin{array}{l}\text { Early social network mobilization and } \\
\text { involvement }\end{array}$ \\
\hline & Openness in communication \\
\hline & $\begin{array}{l}\text { Early education and raising awareness by } \\
\text { healthcare professionals }\end{array}$ \\
\hline & Reciprocity in relationships \\
\hline
\end{tabular}


my husband with dementia. I always want to maintain a part of my own life and interest for others and in life itself ... and I am also afraid that it is not good for me. I always have been a very active person with lots to talk about. And I think it is also not good for my husband because then I might put pressure on him." (P, 4)

\section{Barriers to seek support}

Despite the need for support, spousal caregivers often experienced barriers to ask for support because of practical factors, such as time and distance, as well as personal values or norms. For example, autonomy and independence were values deeply rooted in one's upbringing. Spouses often insisted on managing their own care tasks to prevent them from being a burden to others:

"Why it is difficult? Because I am a person capable of arranging my own affairs. And then you somehow have to be humble. Do you want to do that? (Sigh) Argh ... and then I think I can do it myself. I prefer that." $(\mathrm{P}, 6)$

Another hindering factor for asking for support was that spouses delayed asking for help to protect the PwD and maintain their long-lasting bond:

"We'll cross that bridge when we get to it. I can manage it at the moment. Therefore, in my view you don't ask other people for a favor. I think we are married for 52 years, if you truly love someone, then everything is possible. If you don't love someone, it would be very difficult to carry on." $(\mathrm{P}, 10)$

Furthermore, spouses experienced misunderstanding with respect to their situation, leading to feelings of stigmatization, isolation or loneliness:

"It is in my nature, trying to prevent burdening others with the problems of my wife. On the one hand, I don't want to burden others and simultaneously if you rely on that help, people will sympathize with my wife and will really treat her likewise (as a PwD). I want to avoid or postpone this. I don't want to hide things, but I want to keep people from offering support that is disproportionate. Then, you stop addressing her own abilities. Additionally, I don't want to burden my children because I feel I can manage the situation myself." $(P, 1)$

"Few people show understanding; they can be counted on one hand. Because 
they have not experienced the same situation, you avoid those people or you stop talking about it at all. It makes no sense. Also, they don't need that, you notice that, is even the case in the family. They say my mom this, my father that ... and they don't even let you finish talking. Then, I wonder... don't you understand? I want to tell something about myself. Thus, I automatically keep silent." $(P, 3)$

\section{Facilitators to seek support}

In addition to the barriers to seeking support, several facilitators were identified. Especially because of the growing awareness that support is essential, positive support experiences and good quality relationships stimulated support-seeking behavior:

"Let's say the longer it takes, the easier it gets to call on people. I can't do everything ... I have to accept things; I have no choice, so to say. That is what I call a lesson in humility, it was necessary, I saw and felt that I had to be wise and had no choice. So, that means that I can ask for help more easily." $(P, 4)$

"It changed, when I was ill myself, I noticed that people spontaneously would call me and say: 'I heard you are ill, can I do something for you?' If I had not experienced that, it would be more difficult for me. I am not that kind of person. I am used to fighting my own battles, that's part of me, as long as I can do it, I will do it myself. Now I see, it is getting easier. I think experience is the best teacher. The next time I will not be afraid to ask." $(\mathrm{P}, 6)$

In sum, spousal caregivers often did not acknowledge support needs because they were afraid to lose autonomy or put a burden on others. Additionally they were confronted with feelings of loneliness and stigma due to changing relationships and incomprehension by individuals in the environment. However, several caregivers reported positive experiences that changed their willingness to seek support earlier.

\section{Network perspectives on supply of social support}

People in the social environment were often willing to help. They aimed to relieve the pressure for the spousal caregiver, and they considered support to be an enriching experience: 
"She always has been busy, now you can do things for her...especially provide emotional support. I would like to bring her outside her safe environment, to walk with her and talk with her. Then, you can give something back and make her life a little bit better. Especially by emphasizing emotional support, you can enrich life. We could lower the threshold by emphasizing that we don't doubt his abilities, but we want to do something fun to unburden and add something." ( $\mathrm{N}$, 13, daughter-in-law)

\section{Barriers to offering support}

Nevertheless, providing support was hampered because the social network members often had no insight into what kind of support was needed at which moment in time:

"There is kind of a 'debt of honor, like my mother-in-law cared for him for years, now the roles are reversed and he protects her very strongly, but imagine my father-in-law won't be there anymore. We don't know what is happening, we don't know which care strategies he uses, what he did. I was shocked that there is no plan for when he cannot be there anymore. So, I mentioned this a few times to my brothers-in-law, talk about this together how to break through the shield." $(\mathrm{N}, 13$, daughter-in-law)

"For us, the point is, we are searching for the most appropriate moment and the best approach to offer support. We are confronted with this for the first time, and this is our first experience." ( $\mathrm{N}, 12$, son)

Moreover, the involved network members were afraid of violating privacy or stigmatizing the spouse in the caregiver role:

"The problem or the only danger I see is that I don't want to intervene in their daily life with each other. So, he has to be open for support. I mean that remains the point." ( $\mathrm{N}, 12$, son)

Other hindering factors were rejection of support by the spouses or PwD and emotional confrontation through role change inherent to dementia:

"We ran into a wall, my sister and I. So, we said we can't do anything. We noticed aversion. She is suspicious that my sister arranges things behind her back. Since we ran into this wall, we changed to a hands-off approach and just 
try to be son and daughter and nothing else." (N, 27, son)

\section{Facilitators to offering support}

Several factors contributed towards the provision of support, especially a prevailing attitude that you offer support for better or for worse. Other factors include a positive focus towards providing support, good quality relationships, and observed health decline:

"Whether or not the caregiver can ask for help depends on the relationship of the caregiver with the environment and vice versa. In my situation, we are the youngest in this neighborhood. We are open-minded; there are two ways of looking at people: you can just look at people and say hello or you can really care about people, you know what I mean?" (N, 11, neighbor)

In sum, although the involved network members were willing to offer support, there was a lack of insight into the exact type of support needs. They faced practical limitations as well as confrontation and rejection. A positive and open view towards caring as being a normal process and a focus on opportunities, instead of limitations, facilitated involvement.

\section{A mismatch between supply and demand of informal support}

The data demonstrated a mismatch between the supply and demand of social support (Fig. 2). Frequently, intentions to ask for support or provide support were not translated into action. This mismatch was strengthened by a cognitive bias observed in the spouses and their network members. They tended to think for others and might hold incorrect or unverified presumptions concerning each other's needs and intentions, which prevented tangible support seeking and support provision:

"We contemplate things in advance: what should I do? On which day? Can I do it? Am I able to live up to what I promised to do? And he might think exactly the same ... such as how will they treat my love, they are already busy, they have this and that and already have enough worries." ( $N, 13$, daughter-in-law)

"People might think she is able to handle the situation. That also makes a difference. I think if you let your head hang down and are shaky, then people will 
come over more quickly. That might be possible. However, I am not like that." $(\mathrm{P}, 3)$

Spouses and network members did not always communicate their thoughts and feelings. Consequently, they experienced unfulfilled expectations leading to reciprocal disappointment:

"One of the biggest disappointments was with one of our friends. They were the first couple I told about the situation when my husband was present. They both work in dementia care settings and then you expect ... and I think that is wrong to expect that they would just come by ... like 'How are you doing and can we help you? We might take your husband for a walk so you have some spare time.' I think I expected too much. However, if I asked them, they probably would help. However, other stupid things happened. Then, I think I wouldn't have expected that. So there I experience some kind of disappointment." $(P, 4)$

In sum, the observed mismatch in care networks is strengthened due to cognitive biases. People make unverified presumptions about each other's needs and abilities, leading to unfulfilled expectations and disappointment.

\section{Openness in communication to repair the imbalance}

Bridging the gap between spousal caregivers and their social network members emphasized that more openness in communication is required in both informal social networks and formal healthcare settings in an earlier phase of the disease. Sharing experiences, needs and wishes and mapping support opportunities within (online) family networks might increase involvement and awareness of support needs:

"I think it is important to share information, especially to stimulate each other. Let me put it this way, I told you about an online tool to schedule a date. That, in my opinion, is perfect. I imagine I would be the organizer of the network of my mother-in-law. And I had asked someone to go somewhere with her; then, I send that specific person a link asking, 'How did it go?' Following completing the link and posting a small blog with her experience, she would be able to read messages from the others. This way you might trigger an interaction with each other. In my opinion, it is the first priority to share things. Additionally, that would 
have the intention to enrich the life of others and share that with others. For example, a beautiful moment can be shared by ten people, and those ten beautiful moments can in turn inspire other people." ( $\mathrm{N}, 13$, daughter-in-law)

"At an early stage, you must start building a network. Get people involved before it becomes essential. In that way, a network will be in place when you need it most. Look, if you must start building a network when you already really need it, then it is more difficult for the people who have to provide the support." $(\mathrm{N}, 20$, daughter)

Additionally, healthcare professionals should play a role in creating awareness that support is essential. The caregivers tend to accept an independent expert opinion more easily as opposed to informal advice from a close network member:

"It should not come only from informal helpers. However, also from let's say the medical side, they have to manage that caregivers are triggered to think about which persons can help you, with what kind of tasks and would you be able to share your support needs. When there is one person to help, then it might get more easy to examine which other people can be involved to set the stage." ( $\mathrm{N}, 11$, neighbor)

Moreover, the relationships based on equality and openness were highly valued. The caregivers not only want to depend on the kindness of others, but they also wish to return something for their support:

"I find it difficult to offer support because the caregiver is reluctant. She does not want to ask for support. She wants to maintain our relationship as it used to be. That is a sore point. 'You can lead a horse to water, but you can't make him drink.' My friend wants to continue our relationship and needs the enjoyment our friendship offers. She does not want to be judged as being a burden or a person in need. She wants to maintain an equal friendship." ( $\mathrm{N}, 19$, friend)

In sum, to resolve the observed mismatch, it seems that an openness in communication in both informal and formal care networks is required. Furthermore, to maintain quality care relationships, equality and reciprocity are prerequisites. 


\section{DISCUSSION}

\section{A model of the mismatch between supply and demand of support}

The aim of this study was to explore the needs, perspectives and experiences regarding informal support of both spousal caregivers and social network members. Furthermore, we examined the barriers and facilitators regarding the supply and demand of informal support. Four themes were identified: (1) spousal caregivers had different support needs, such as the need for emotional support, practical support, respite care or company and relaxation. Such support enhanced the caregivers' social integration and the feeling of being meaningful in daily life. (2) Social network members were willing to supply support because support was considered to be a rewarding experience that led to enriched relationships. (3) Nevertheless, a mismatch between the supply and demand of informal support was demonstrated. Although the spouses reported explicit support needs and the network members were often willing to help, frequently no actual support was delivered because of the outlined barriers. The hindering factors were partly similar to those identified in earlier research, such as a fear of stigma ${ }^{16}$, or negative attitudes towards accepting support, such as a sense of responsibility and unwillingness to relinquish support to others. ${ }^{17}$ The identified mismatch was strengthened by an observed cognitive bias. Both the spousal caregivers and the social network members tended to think for others and exhibited unverified and sometimes incorrect thoughts concerning each other's intentions. Consequently, both parties may not explicitly communicate their support wishes and opportunities because of a concern for an anticipated burden, rejection or violation of privacy, thus causing disappointment and unfulfilled expectations. (4) Our data highlighted that open communication in formal and informal care systems could repair the observed mismatch between the supply and demand of informal support.

\section{Practical implications}

Our results suggested that it is essential that healthcare professionals raise awareness regarding the importance of mobilizing informal support at an earlier stage of the disease. Previously, caregivers found it difficult to acknowledge and accept care needs in an early phase of the disease. ${ }^{15}$ The healthcare professionals should not only emphasize the significance of social support but also focus on the positive aspects of support and reciprocal interaction so that caregivers can come to terms with their social network involvement. 
Our findings revealed that cognitive biases play a role in support behavior; the caregivers reported thinking for other social network members and vice versa. According to Beck's cognitive theory, people can become susceptible to interpreting the intentions of others in a distorted or biased way because meanings are derived from personal experiences and the way people structure the world. $^{18,19}$ Our findings confirmed that caregivers make unverified presumptions. As shown in previous studies, multi-component family meetings ${ }^{20}$ or remote interventions ${ }^{21,22}$ might effectively improve support and open communication to prevent these cognitive biases. Additionally, support interventions may include cognitive behavioral techniques that address maladaptive thoughts regarding caregiver support. For example, cognitive restructuring and positive reappraisal techniques may shed a more positive light on seeking informal support, such as the realization that providing or asking for support does not only impose a burden or stigma but, in contrast, might lead to a reciprocal enrichment of relationships and increased social functioning. Consistent with this finding, future caregiver intervention research might increasingly focus on strengthening aspects of social health. The newly developed concept of social health emphasizes people's capacity to fulfill their potential and obligations, manage their life with some degree of independence and engagement in social activities, despite their medical condition. ${ }^{23}$ From this perspective, health is a dynamic balance between opportunities and limitations. Our data confirmed that participation in social activities parallel to the process of caregiving are highly valued and that a positive focus on opportunities, rather than limitations, might facilitate positive social interactions and supportive behavior.

\section{Methodological strengths and limitations}

This study is unique in integrating the perspectives of both the spousal caregivers and the involved network members. By using an ecogram as a sensitization technique, we collected more in-depth information on the social network processes. The spousal caregivers were triggered to reflect on and disclose present and past experiences within the context of their social network, which guided the interview process. ${ }^{14} \mathrm{~A}$ drawback was that interviewing acquaintances might have prompted a response bias. However, the interviewer explicitly emphasized that the data would remain anonymous. To increase the homogeneity of our sample, we only included spousal primary caregivers from 
the southern regions of the Netherlands. Although the literature indicated that the majority of dementia caregivers are spouses, ${ }^{24}$ additional perspectives of other primary caregivers with more diverse backgrounds would have enhanced the generalizability of the results. Previous studies have demonstrated that social support differs across ethnicities and cultures. ${ }^{25}$ Therefore, it might be interesting to repeat this study in another cultural context.

\section{Conclusion}

Our novel method was highly effective in identifying integrated perspectives on social support in dementia caregiver networks. We identified a model explaining the mismatch between supply and demand of support. A new finding is that openness in communication might repair the observed mismatch. This finding underscores the importance of early education and social network interventions to facilitate positive interactions. Innovative online communication tools, such as social media platforms, might be a new venue for improving the openness in communication and encouraging positive involvement in dementia care networks. This may yield opportunities to overcome the experienced barriers, such as the fear of burdening and stigmatizing others, and enhance access to social support. 


\section{References}

1. Schulz R, Martire LM. Family caregiving of persons with dementia: prevalence, health effects, and support strategies. Am J Geriatr Psychiatry 2004; 12(3): 240-9.

2. Au A, Lai M-K, Lau K-M, et al. Social support and well-being in dementia family caregivers: The mediating role of self-efficacy. Aging \& Mental Health 2009; 13(5): 761-8.

3. Kiecolt-Glaser JK, Dyer CS, Shuttleworth EC. Upsetting social interactions and distress among Alzheimer's disease family care-givers: a replication and extension. Am J Community Psychol 1988; 16(6): 825-37.

4. Coen RF, O'Boyle CA, Coakley D, Lawlor BA. Dementia carer education and patient behaviour disturbance. Int J Geriatr Psychiatry 1999; 14(4): 302-6.

5. Gold DP, Cohen C, Shulman K, Zucchero C, Andres D, Etezadi J. Caregiving and dementia: predicting negative and positive outcomes for caregivers. Int J Aging Hum Dev 1995; 41(3): 183-201.

6. Brodaty $\mathrm{H}$, Thomson $\mathrm{C}$, Thompson $\mathrm{C}$, Fine M. Why caregivers of people with dementia and memory loss don't use services. Int J Geriatr Psychiatry 2005; 20(6): 537-46.

7. Wolfs CA, de Vugt ME, Verkaaik M, Verkade PJ, Verhey FR. Empowered or overpowered? Service use, needs, wants and demands in elderly patients with cognitive impairments. International journal of geriatric psychiatry 2010; 25(10): 1006-12.

8. Mackenzie J. Stigma and dementia: East European and South Asian family carers negotiating stigma in the UK. Dementia: The International Journal of Social Research and Practice 2006; 5(2): 233-47.

9. Pollitt P, Andersont I, O'Connor D. For better or for worse: The experience of caring for an elderly dementing spouse. Ageing and Society 1991; 11(04): 443-69.

10. Fortinsky $\mathrm{RH}$, Downs $\mathrm{M}$. Optimizing person-centered transitions in the dementia journey: A comparison of national dementia strategies. Health Affairs 2014; 33(4): 566-73.

11. Rosow K, Holzapfel A, Karlawish JH, Baumgart M, Bain LJ, Khachaturian AS. Countrywide strategic plans on Alzheimer's disease: Developing the framework for the international battle against Alzheimer's disease. Alzheimer's \& Dementia 2011; 7(6): 615-21. 
12. Boeije H. Analyseren in kwalitatief onderzoek [Analysis in qualitative research] Boom; 2005.

13. Baars H, Uffing J, Dekkers G. Sociale netwerkstrategieën in de sociale psychiatrie. Houten/Antwerpen: Bohn Stafeu van Loghum 1990.

14. Visser FS, Stappers PJ, Van der Lugt R, Sanders EB. Contextmapping: experiences from practice. CoDesign 2005; 1(2): 119-49.

15. Boots LM, Wolfs CA, Verhey FR, Kempen GI, de Vugt ME. Qualitative study on needs and wishes of early-stage dementia caregivers: the paradox between needing and accepting help. International Psychogeriatrics 2015; 27(06): 927-36.

16. Clement S, Schauman O, Graham T, et al. What is the impact of mental health-related stigma on help-seeking? A systematic review of quantitative and qualitative studies. Psychol Med 2015; 45(1): 11-27.

17. Greenwood N, Smith R. Barriers and facilitators for male carers in accessing formal and informal support: A systematic review. Maturitas 2015; 82(2): 162-9.

18. Beck AT. Cognitive therapy and the emotional disorders: Penguin; 1979.

19. Bless H, Fiedler K, Strack F. Social cognition: How individuals construct social reality: Psychology Press; 2004.

20. Drentea P, Clay OJ, Roth DL, Mittelman MS. Predictors of improvement in social support: Five-year effects of a structured intervention for caregivers of spouses with Alzheimer's disease. Soc Sci Med 2006; 63(4): 957-67.

21. Boots LM, de Vugt ME, van Knippenberg RJ, Kempen GI, Verhey FR. A systematic review of Internet-based supportive interventions for caregivers of patients with dementia. Int J Geriatr Psychiatry 2014; 29(4): 331-44.

22. Dam A, de Vugt ME, Klinkenberg IP, Verhey FR, van Boxtel MP. A systematic review of social support interventions for caregivers of people with dementia: Are they doing what they promise? Maturitas 2016.

23. Huber M, Knottnerus JA, Green L, et al. How should we define health? Bmj 2011; 343: d4163.

24. Aneshensel CS, Pearlin LI, Mullan JT, Zarit SH, Whitlatch CJ. Profiles in caregiving: The unexpected career: Academic Press; 1995.

25. Valle R, Yamada AM, Barrio C. Ethnic differences in social network helpseeking strategies among Latino and Euro-Aamerican dementia caregivers. Aging Ment Health 2004; 8(6): 535-43. 




\section{CHAPTER 4}

Qualitative study on needs and wishes of early-stage dementia caregivers: the paradox between needing and accepting help

International Psychogeriatrics, 2015

Lizzy M.M. Boots, Claire A.G. Wolfs, Frans R.J. Verhey, Gertrudis I.J.M. Kempen \& Marjolein E. de Vugt 


\section{ABSTRACT}

Background: Early individualized interventions for informal dementia caregivers can prevent overburdening in the later stages. However, the needs of early-stage dementia caregivers (EDC) remain largely unknown. This study aimed to explore the needs and wishes and need for care of EDC to maximize the benefit of potential programs for EDC and tailor interventions accordingly.

Methods: Four focus group interviews with 28 informal caregivers of people with dementia (PwD) were analyzed using inductive content analysis. Both EDC and caregivers in the later stages were included to compare perceived EDC needs from different points in the caregiver career.

Results: Four themes were identified: the early-stage needs paradox, barriers in acceptance, facilitators in acceptance and a transition from loss to adaptation. The retrospective view provided by later-stage caregivers differed from the view of EDC; EDC struggled with acknowledging needs due to fear of stigma and low acceptance. EDC stressed the importance of acceptance as a prerequisite for adequate adaptation, but were hindered by lack of knowledge, difficulty acknowledging changes and focus on loss. In contrast, better understanding of the disease, increasing personal time, structuring ones day and using appropriate humor can reduce negative communication, increase positive encounters and caregiverconfidence, contributing to positive interaction with the care recipient and an increase in well-being.

Conclusions: Early therapeutic interventions could help caregivers identify their needs, increase knowledge about changes in roles and relationship reciprocity, and focus on enhancement of the positive, intact experiences to prevent caregiver burden. 


\section{INTRODUCTION}

The ageing of the population is expected to lead to a substantial increase in the prevalence of dementia ${ }^{1}$ and significant concerns exist about whether the current labor force can cope with such an increased future demand. ${ }^{2}$ Consequently, the care for people with dementia (PwD) will largely depend on informal caregivers, usually spouses. Informal caregivers often provide years of extensive care for their loved one, and must adapt to continuously changing and increasingly demanding circumstances. This challenging role may cause chronic stress, making caregivers highly vulnerable for being overburdened and developing psychological symptoms such as depression and anxiety. ${ }^{3}$ Even in the very early stages, for example in the Mild Cognitive Impairment $(\mathrm{MCl})$ phase, caregivers often experience distress and depressive symptoms. ${ }^{4}$

Clinicians, researchers and Alzheimer societies stress the importance of a timely diagnosis in dementia, and this is also advocated by most international dementia strategies. ${ }^{5} \mathrm{~A}$ timely diagnosis in the early or prodromal phase, may offer caregivers the opportunity to prepare for their future caregiving task in the early stages of the disease and prevent high levels of burden in the later stages. ${ }^{6}$ Early intervention and support for caregivers can be effective in reducing strain and may delay institutionalization of the person with dementia. ${ }^{7,8}$ As levels of stress and burden are relatively low in the early, including prodromal, stages of dementia, interventions at that time present an opportunity for professionals to help caregivers cope with an insecure future and prepare themselves for the possibility of further decline and dependency. ${ }^{9}$ However, the specific needs of early-stage dementia caregivers (EDC) remain largely unknown. ${ }^{6}$ The needs of PwD and their caregivers in later stages of the disease have already been examined extensively. These include the need for emotional, social and financial support, information about the disease and information about appropriate care facilities. ${ }^{10-13}$ Caregivers also report a lack of competence to provide care and difficulty to plan ahead for their relative's future care needs. ${ }^{12}$ This demonstrates the importance of supporting EDC to prepare them for their difficult future task.

To maximize the benefit of EDC interventions, the needs of caregivers should be carefully assessed to determine whether and which interventions in this stage are desired and to tailor interventions accordingly. ${ }^{14}$ The present study therefore focuses on an in-depth analysis of the needs of EDC. More specifically, we sought to (1) gain insight into the problems, needs and wishes that caregivers of PwD encounter during 
the early stages of the disease, (2) explore if an early-stage intervention for dementia caregivers would be helpful and (3) explore which factors influence caregivers' perspectives on early-stage need for care.

\section{METHODS}

\section{Study participants}

Purposeful sampling was used to describe the EDC needs that cut across participants with a range of variation on disease severity, gender, age, and relationship with the relative. ${ }^{15}$ The maximum variation sampling strategy was also successfully used in previous research on informal caregiver needs ${ }^{12}$ and allowed us to select participants with these different characteristics to look for common patterns and capture the EDC perceived needs from different points in the caregiver career. $A$ minimum of four focus group interviews with 6-8 participants per group was planned; sampling ended when data saturation was achieved, e.g. no new themes emerged. ${ }^{15}$ Between August 2012 and January 2013, 40 informal caregivers were recruited; 14 early, including prodromal, stage dementia caregivers were purposively selected by professional caregivers from the pool of informal EDC associated with the (1) Memory Clinic of the Maastricht University Medical Center; and 26 later stage dementia caregivers were purposively selected by professional caregivers from the pool of later stage informal caregivers associated with the (2) Alzheimer Café Roermond (monthly meeting spot for people with dementia (PwD), caregivers and professionals); and (3) Regional Committee Healthcare ( $\mathrm{RCH})$ Proteion, which is a care home for older people. Participants were included if they were (1) the informal caregivers of a person with dementia or prodromal dementia $(\mathrm{MCl}$ due to $\mathrm{AD}$, based on the etiological diagnosis expected to convert to dementia), and (2) involved in the daily care of the care recipient at least once a week. The inclusion of caregivers affiliated with different institutions fostered maximum variation of the sample. Twentyfive informal caregivers agreed to participate. Eight caregivers declined participation due to a busy caregiving schedule; six caregivers were not interested in a group meeting and one caregiver declined due to work-related commitments. Three secondary informal caregivers of already represented care recipients requested to join the primary participating caregiver in the focus group interview. The Medical Ethics Committee of the Maastricht University Medical Center approved this study (\#12-4-059). 


\section{Procedures}

Focus group interviews were used to explore caregiver perceptions, opinions and beliefs, because focus groups are an ideal method to reveal various perspectives on a topic and to uncover new insights and unanticipated issues. ${ }^{16}$ Four focus group interviews were scheduled, applying triangulation of sites: two groups ( $A$ and $B$ ) of six participants in the city of Maastricht and two groups (C and $D$ ) of seven and nine participants respectively in the city of Roermond (both cities are located in the southern part of the Netherlands). Methodological guidelines suggest that most issues around a research topic will be captured after the fourth group discussion. ${ }^{16}$ The point of saturation was checked after the fourth group discussion independently by two authors (LMMB, CAGW) to confirm that a sufficient number of focus groups had been conducted as no new themes emerged. Written informed consent was obtained prior to participation in the study. This included explaining the procedure to be followed and the roles of the moderator (MEdV) and assistant (LMMB; taking additional notes). Participants were encouraged to share their expertise (e.g. life experiences) and to view the focus groups as a social event as a means of encouraging open discussion. ${ }^{17}$ A semi-structured guide was used during the focus group sessions. It included topics derived from clinical practice that identified needs and wishes expressed by EDC to prevent possible overburdening in the later stages, EDC need for care or experiences with received care, and EDC need for communication of care. Brief summaries of the key points were made throughout the focus group sessions as a method for obtaining participant verification. ${ }^{16}$ All focus group interviews were videotaped, and tapes were stored in a locked area. Triangulation of within-method data sources was applied ${ }^{15}$; video recordings were used in addition to the field notes taken by the assistant to ensure identification of potentially relevant non-verbal information or cues presented by the participants. Interviews lasted approximately 120 minutes.

\section{Context-mapping approach}

For the preparation and execution of the focus group sessions a context-mapping approach was used. This approach presumes that information about the context of people's interactions in their everyday life is needed to reveal experiences and emotions in a group environment. Prior to the interview, participants were asked to think, reflect, and explore aspects of their personal context and current situation. ${ }^{18}$ Therefore, we provided them with packages comprising a booklet to capture parts of 
their history and current life with their spouse two weeks before the interview. To elicit thoughts and feelings of participants during the group meetings, participants were asked to elaborate on their experiences based on generative techniques such as collage making (Figure 1). ${ }^{18}$ This indirect approach was chosen to minimize the risk of increasing feelings of distress caused by directly confronting caregivers with the memory impairment of their loved one. ${ }^{19}$ The material was developed in collaboration with an expert in context-mapping to verify its quality and suitability for the present study.

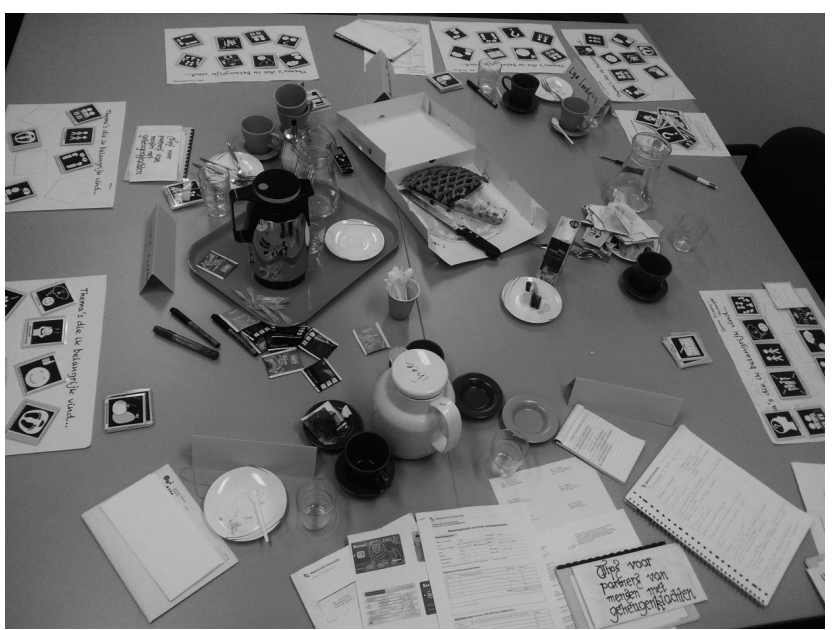

Figure 1. Collages of themes created by participants during a focus group

\section{Data analysis}

The videotapes of all focus group interviews were transcribed verbatim. Analyst triangulation was applied ${ }^{15}$; the transcripts and observations combined with additional notes taken during the focus group interviews and the collected context mapping materials were analyzed independently by two authors (LMMB, CAGW) using the qualitative analysis software ATLAS.ti (version 7.0). An inductive content analysis approach was adopted, ${ }^{20}$ in which common themes and categories emerge using inductive reasoning and constant comparison. The texts were thoroughly read and open codes were applied to describe all aspects of the content. ${ }^{21}$ Codes referring to the same phenomenon were grouped into categories and these categories were grouped into higher-order themes. Categories and themes were combined into general statements to describe the phenomenon. ${ }^{20}$ Discrepancies in coding and interpretation were discussed in a meeting together with a third author (MEdV) to 
reach consensus regarding the categories and themes. The video recordings were compared with the written transcripts to be able to identify potentially relevant additional non-verbal information or cues presented by the participants. Saturation was established as none of the categories or themes shown in Table 2 (Results) emerged after the third focus group interview. The quotations described in the results section to clarify the major themes were based on those categories that were coded most often by authors LMMB and CAGW. Quotes were selected by the first author (LMMB), verified by the second author (CAGW) and checked for relevance by all authors.

\section{RESULTS}

\section{Participants}

Table 1 represents the characteristics of the 28 informal caregivers (mean age $=63.6$ years) that participated in the study. Six participating caregivers (children $n=2$, children-in-law $n=2$, spouse $n=1$, friend $n=1$ ) were responsible for the care of three care recipients. The majority were females $(75 \%)$, spouses $(78 \%)$, and lived together with the care recipient $(75 \%)$. Care recipients $(n=25)$ were diagnosed with Mild Cognitive Impairment due to AD (32\%), Alzheimer's dementia (44\%), and other dementias $(24 \%)$ and were in the (very) early stages of the disease $(52 \%)$ or had more advanced dementia (48\%). The duration of diagnosis ranged from $0.5-10$ years. Two groups comprised spousal caregivers only and two groups included a mixture of both spousal caregivers and other informal caregivers. Both authors (LMMB and CAGW) independently concluded that the quotes of prodromal dementia caregivers ( $\mathrm{MCl}$ due to $\mathrm{AD}$ ) and early-stage dementia caregivers were covered by the same codes and categories. Therefore, the results of this study did not separate $\mathrm{MCl}$ as a separate group from early-stage dementia caregivers (EDC). EDC and caregivers in the later stages were equally represented in all groups.

\section{Focus groups}

Four major themes covering the problems, needs and wishes experienced in the early stage of caregiving were identified: (1) The early-stage needs paradox; (2) Barriers in the acceptance process; (3) Facilitators in the acceptance process; and (4) Shift of focus: from feelings of loss to adaptation. An overview of the categories and themes is provided in Table 2 and Figure 2, and elaborated below by means of quotations. Each quotation is assigned a code; the letter stands for the location and 
sequence of the focus group interview, while the number indicates the specific participant. After each code caregivers are assigned to either the EDC or LDC group; EDC stands for early-stage dementia caregiver and LDC stands for later-stage dementia caregiver, as based on the clinical judgment of the referring professional caregiver. Both authors (LMMB and CAGW) concluded that no discrepancies could be discovered between the written transcripts and potentially relevant non-verbal cues brought forward by the participants.

Table 1. Background characteristics of the caregivers $(N=28)$ and the care recipients $(N=25)$

\begin{tabular}{|c|c|c|}
\hline AGE (MEAN) & 63.6 YEARS & \\
\hline \multirow[t]{2}{*}{ Gender } & Men & $7(25 \%)$ \\
\hline & Women & $21(75 \%)$ \\
\hline \multirow[t]{5}{*}{ Relationship to the care recipient } & Spouse & $22(78 \%)$ \\
\hline & Child & $2(7 \%)$ \\
\hline & Child-in-law & $2(7 \%)$ \\
\hline & Sibling & $1(4 \%)$ \\
\hline & Friend & $1(4 \%)$ \\
\hline \multirow[t]{2}{*}{ Living together with care recipient } & Yes & $21(75 \%)$ \\
\hline & No & $7(25 \%)$ \\
\hline \multirow[t]{5}{*}{ Care recipient diagnosis } & $\mathrm{MCl}$ due to $\mathrm{AD}$ & $8(32 \%)$ \\
\hline & $A D$ & $11(44 \%)$ \\
\hline & Vascular Dementia & $3(12 \%)$ \\
\hline & Parkinson & $1(4 \%)$ \\
\hline & Dementia NOS ${ }^{a}$ & $2(8 \%)$ \\
\hline \multirow[t]{2}{*}{ Stage of the disease ${ }^{b}$} & (Very) early stage & $13(52 \%)$ \\
\hline & Later stage & $12(48 \%)$ \\
\hline \multirow[t]{4}{*}{ Care recipient years of diagnosis } & 0.5 & $5(20 \%)$ \\
\hline & $1-3$ & $9(36 \%)$ \\
\hline & $4-6$ & $10(40 \%)$ \\
\hline & $7-10$ & $1(4 \%)$ \\
\hline
\end{tabular}

${ }^{\mathrm{a}} \mathrm{NOS}=$ Not Otherwise Specified

${ }^{\mathrm{b}}$ based on clinical judgment of the referring professional caregiver 
Table 2. Overview of categories and major themes

\begin{tabular}{ll}
\hline THEMES & CATEGORIES \\
\hline Early-stage needs paradox & Caregiver needs in early stages \\
& $\begin{array}{l}\text { Difficulties concerning roles and relationship } \\
\text { changes }\end{array}$ \\
& Early-stage available support \\
& Denial and difficulty accepting \\
Hindering factors in the acceptance process & Loss and grief \\
Facilitating factors in the acceptance process & Future expectations \\
& $\begin{array}{l}\text { Acceptance process } \\
\text { Need for information }\end{array}$ \\
Shift of focus: from loss to adaptation & Support and tips \\
& $\begin{array}{l}\text { Maintaining a good quality of life together } \\
\text { with patient }\end{array}$ \\
& Adapting care to personal situation \\
& Individual differences \\
\hline
\end{tabular}

THE CAREGIVER NEED PARADOX

BARRIERS

FOCUS ON

LOSS

NEGATIVE NEGATIVE

INTERACTION EMOTIONS

LOW

\section{FACILITATORS}

FOCUS ON POSITIVE
ACCEPTANCE HIGH
EARLY STAGE NEEDS

NOT ACKNOWLEDGED
EARLY STAGE NEEDS

ACKNOWLEDGED

Figure 2. Schematic representation of the early-stage need paradox and acceptance process 


\section{Early-stage needs paradox}

Retrospectively looking back at the early stages, caregivers in the later stages stressed the need for care that would have facilitated their acceptance process. They indicated that they would have benefited from talking to someone who could understand their situation and recognize their problems earlier. They also stressed the importance of timely guidance tailored to their individual needs as an intervention that would have helped them cope with the situations and worries they experienced.

"Now I think it would have been better for me if I received some guidance in the beginning, just someone helping me find my way. Not telling me: 'this is what you can expect', but asking me what I experience or worry about and helping me how to cope with certain situations or incidents. I had to figure that out all by myself and sometimes that worked out but sometimes it really didn't." (D6, LDC)

"I would have liked to have received some information that was important for me at that moment. I hear a lot of different stories and some do apply to our situation but others really don't. It's all so different and it needs to be relevant for your situation at that specific time" $(A 3, L D C)$

However, EDC did not recognize the needs underlined by the later stage caregivers. EDC found it difficult to accept help, because they did not want to stigmatize their situation or did not want to be seen as in need of professional help.

"I don't like to be called the 'caregiver', that makes me feel like a patient and I'm not. He's my husband and of course I take care of him. I don't like to feel pathetic, like I have a problem." (B6, EDC)

Also, the information that was available for caregivers had a negative, stigmatizing nature, which made it more difficult for EDC to identify with.

"When I go online to find more information about certain things, I get so much information and especially negative information. It seems that I can't find anything positive. That creates a negative image and that's what I take home after reading. All that information really emphasizes the negative... but it's not all negative" (B1, EDC)

Despite their difficulty in acknowledging needs and accepting help, EDC did encounter many changes and struggles and found it difficult to cope with and accept the situation. 
"I think it's exhausting that my husband forgets so many things. He keeps asking the same thing over and over, places things different than I'm used to or just forgets where he located it. This sometimes irritates me, and afterwards I regret feeling irritated. I don't know... I don't like the fact that I might need help." (A2, EDC)

In sum, a clear paradox existed between the retrospective view provided by caregivers in the later stages of the benefits that early care tailored to personal needs would facilitate the acceptance process and the EDCs' lack of acknowledging a need for care due to difficulties in accepting the changes, fear of stigma and the stigmatizing character of the available information.

\section{Barriers in the acceptance process}

Acceptance was seen as a prerequisite for caregivers to cope with the changes of the disease. However, besides fear of stigma, several factors hindered the acceptance process and prevented EDC from being able to find an effective way to handle the changing situation. Not acknowledging the disease and a lack of knowledge about the manifestations of the disease seriously hindered the acceptance process.

"As long as you deny it you can't cope with it. As soon as you accept it, you can learn to turn to yourself and think: 'how can I cope best with this new situation?" (C6, LDC)

"To cope with the diagnosis you need to know what to do and what not to do. Only when you know how to do it right you can learn to accept it. That's a difficult process, before you can set the problem aside for a bit and accept it, but once you've accepted it you are not always thinking about it and you can get on with your life." $(A 3, L D C)$

Because of this lack of knowledge about the possible disease manifestations, EDC often took the changes in behavior personally, which led to insecurities, unnecessary misunderstandings and discussions.

"I really have to get used to the fact that his head is busy, that he thinks differently. It always escalates when I go on, but it's really hard to understand that he sees it differently. I often push through and we end up in discussion." (A4, EDC)

Furthermore, lack of knowledge in people surrounding the care recipient and caregiver caused EDC to feel insecure about their own actions. 
"It's just the worst feeling in the world when others say: 'what's wrong with him, he looks just fine?' That devastates me, then I start to question myself." (D3, EDC)

Caregivers were confronted with the negative feelings and losses because misunderstandings occurred frequently in the early stages. These feelings of loss hindered the acceptance of the disease and its implications for their lives. Consequently, negative emotions got the upper hand.

"I find it very difficult to accept everything and I can't really cope well with the new situation. I also find it difficult what to do with myself... I don't feel like doing anything and I don't enjoy things anymore. That's where I am right now" (D5, EDC)

When negative EDC emotions outweighed the positive, the likelihood of disruptive encounters with the care recipient increased, and this was followed by even more negative emotions. This vicious circle made it increasingly difficult to control the negative interaction with the care recipient.

"The fear of failing and suspicion is constantly present and that makes her restless. The smallest comment can push her over the edge and cause the situation to escalate. You don't want it to, but it happens... all the time. Unfortunately in the beginning it happened daily. That really hurt." (A1, EDC)

In sum, denying the disease, a lack of knowledge and a focus on loss increased negative emotions and feelings of uncertainty in EDC. When negative experiences outweighed positive emotions the acceptance process was furthered hampered.

\section{Facilitators in the acceptance process}

The process of accepting the disease and its changes could be also facilitated. Disease-specific knowledge was identified as crucial in helping caregivers understand the manifestations of the disease and its changeable nature in the early stages. Information helped caregivers avoid taking the changes personally and improved their ability to empathize with the care recipient.

"You need to realize that your spouse is not doing these things on purpose, they happen because of their inability. He is not doing this to hurt me. That really changes the way you look at the situation. If you realize that, you can stay calm and it won't escalate." (B6, EDC) 
Furthermore, caregivers reported that designating personal time was important for maintaining a healthy balance between caring for the care recipients and caring for themselves. Taking a break helped caregivers to recharge their energy level and it improved their ability to cope with the demanding situation.

"Your resilience just decreases during the day. Then, when something happens, I respond irritated and say something wrong. Whenever I feel my resilience declining, I do something to recharge it; I withdraw myself from the situation, we still sit together but I start to read something or do a puzzle while recharging myself and thinking about the fact that it's not her fault. It gives you the time to think about what you're going to say and avoid restlessness or worse..." (A1, EDC)

However, caregivers found it difficult to set aside personal time when the care recipient demanded their attention. Establishing structure and routine in daily life with the care recipient provided personal time for caregivers and simultaneously decreased restlessness and uncertainty in the care recipient.

"I go hiking every week and he knows that. If he starts to get agitated about me leaving I just refer to the calendar and remind him that it's my hiking day today. He doesn't always like that, but he accepts it because it's in our routine." (A3, LDC)

Dementia obliged caregivers to take on different roles in the relationship with the care recipient. This change in roles was difficult to adapt to. Acknowledging the change in roles and relationship decreased negative encounters. This new role as a caregiver demanded a certain level of confidence that could be increased by positive experiences, such as spending time alone, with the care recipient or with people from the caregivers' social network.

"I tried working on my confidence by taking walks and going to the gym and talking to others, just being able to say what's on my mind... that helps." (B5, LDC)

Humor, when appropriate, was also mentioned as an approach that lessened the negative impact of the disease and helped caregivers identify positive rather than negative aspects of living with the care recipient.

'When I do something wrong, she usually says: 'who has Alzheimer's, you or me?' Or when something small goes wrong I call it Alzheimer's light... that kind of sly jokes help lighten the situation." (A6, LDC)

In sum, understanding disease manifestations, applying structure and routine and designating personal time aided the acceptance of the disease and buffered its 
consequences on daily life. Acknowledging the changes in roles and relationship reduced negative communication. Using appropriate humor increased positive encounters with the care recipient and helped caregivers expand their caregiverconfidence.

\section{Shift of focus: from loss to adaptation}

The facilitators in the acceptance process provided caregivers with the opportunity to adjust their expectations and to focus more on the positive aspects such as new or other possibilities in their life together with the care recipient. Their focus shifted from what they had lost to what was still possible with the proper alterations.

"You learn by trial and error. When the same thing happens over and over you start to adjust your expectations so that the situation isn't looking so bad after all." (B5, LDC)

When caregivers focused more on their possibilities, positive feelings increased, and this facilitated positive interactions with the care recipient and increased the wellbeing of both the caregiver and the care recipient.

"You keep on adjusting to what fits his changed needs. Because when he is happy with the situation, I feel that I'm also happier. That really works both ways." (D1, LDC) However, the process of how spousal dyads adapted to their new situation varied by personality, individual coping mechanisms and the premorbid relationship.

"Every man is different and has a different character. And every woman is also different towards her husband. There is no solution that works for everyone." (D9, $\angle D C)$

In sum, acceptance led to adjusting expectations and a focus on possibilities rather than on loss. This created opportunities for positive interaction with the care recipient and an increase in well-being. However, the process of adaptation was different for each individual spousal dyad.

\section{DISCUSSION}

The aim of this study was to explore the needs and wishes of informal caregivers of people in the early stages of dementia from different perspectives in the caregiver career. The analysis revealed four major themes. The first was the early-stage paradox in needs. The retrospective view of caregivers in the later stages on need for early care tailored to personal needs to facilitate the acceptance process contrasted 
with the early-stage dementia caregivers (EDC) view. EDC struggled with acknowledging needs and accepting help due to difficulties in accepting the changes, fear of stigma and the stigmatizing character of the available information. The second theme identified factors hindering the acceptance process. These included denying the changes, lack of knowledge, and a focus on loss. These barriers led to negative emotions and negative encounters with the care recipient and blocked positive emotions. The third theme identified factors facilitating the acceptance process. These include understanding disease manifestations, designating personal time, acknowledging and gradually establishing changes in roles, structuring their time and appropriately using humor. These facilitators appeared to reduce negative communication and increase positive encounters and caregiver-confidence. The fourth theme highlighted the importance of acceptance as a prerequisite for adapting expectations and focusing on possibilities to be able to maintain a positive caregivercare recipient interaction and well-being.

In the current study, no differences were found between prodromal dementia and early-stage dementia caregivers in the assigned codes and categories. It is possible that no differences were seen between these two groups of caregivers because $\mathrm{MCl}$ due to $A D$ can be viewed as the very early stage of dementia. ${ }^{9}$ Also, caregivers are often aware of the condition of their loved one long before the actual diagnosis is disclosed. $^{22}$

Our results suggest that the early needs retrospectively identified by later stage caregivers contradict the needs for care emphasized by EDC, who actually struggled with acknowledging specific needs. It is conceivable that EDC also relate their need for care to their currently experienced objective burden opposed to their subjective burden. Objective burden refers to aspects like the amount of time spent on caregiving and the type and number of caregiving tasks that are performed, often predicted by behavioral problems and impairments in daily function and cognition. ${ }^{23}$ Subjective burden refers to the emotional response of the caregiver to the demands of the situation, such as feelings of incompetence or exhaustion. ${ }^{24}$ In EDC the objective burden might be lower, given the level of impairment and the lower amount of practical and behavioral problems in dementia compared to the later stages of the disease. EDC might experience subjective burden, but this may be more complex to translate into specific needs as compared to objective burden. Brodaty et al. ${ }^{10}$ also 
found that, despite a low level of life satisfaction and high levels of subjective burden, EDC as well as caregivers in the later stages felt that they did not need services or help. In addition, caregiver experienced stressors are often not directly related to their self-reported needs. ${ }^{25}$

Our results propose that the willingness and ability to accept the disease is required to acknowledge needs and can also be seen as a prerequisite to maintain an acceptable level of well-being together with the care recipient. ${ }^{26}$ However, the current study emphasizes the struggle EDC experience with accepting the changes due to the disease and our results showed that several factors could hinder or facilitate this acceptance process. Previous research confirms that disease-specific knowledge and knowledge on roles and relationship changes can increase caregiver confidence and understanding and facilitate acceptance ${ }^{22,26}$ When caregivers manage to accept the changes in reciprocity, they can shift their focus from loss to opportunities. A strong focus on the intact parts of the relationship and life's simple joys may help to increase the number of positive experiences with the care recipient. ${ }^{26}$ This change in attitude enables caregivers to effectively cope with the changes and increase their well-being. ${ }^{27}$ This explanation is in line with the dual-process theory on coping with loss. ${ }^{28}$ This theory proposes that adaptation to chronic or life-threatening diseases is characterized by two types of adaptation, namely loss-oriented or restorationoriented. EDC can be seen as in the early phase of adaptation, where their focus lies more on loss as caregivers first have to appraise a stressor before they can formulate adaptations to it. An increased level of distress and anxiety accompanies this focus on loss. According to the theory, stimulation of the adaptation process towards the restoration-oriented adaptation can lead to an increase in well-being. However, the process of adaptation and level of well-being also depends on individual characteristics of the EDC, such as coping mechanisms, personalities and premorbid relationships. Previous research confirms that even in apparently similar situations, caregiver experiences can differ depending on the interpretation or meaning caregivers apply to their situation. ${ }^{26}$ Also, a negative premorbid relationship is associated with negative caregiver experiences. ${ }^{26,29}$ Negative caregivers were not able to express empathy and compassion because they interpreted behavior of the care recipient as a continuity of what they considered negative behavior in their relationship, as opposed to a manifestation of the disease. In addition, personality characteristics such as neuroticism are associated with a non-adaptive care strategy 
resulting in higher levels of negative emotions such as depression. ${ }^{3}$ It is essential to take these personal characteristics and the premorbid relationship into account when addressing needs in tailored intervention programs. ${ }^{6}$

The current study is not without limitations. First, all focus group interviews were held in the southern part of the Netherlands. As caregiver needs may differ based on practical aspects such as the care organization per region, ${ }^{11}$ this may have threatened the transferability of our results. However, to reduce the effect of local factors peculiar to one setting, triangulation of sites was carried out with different institutions in the southern part of the Netherlands. ${ }^{30}$ Second, the present study did not apply methodological triangulation of qualitative methods, which could have compensated for the limitations of individual interviews and focus group interviews separately. The use of solely focus group interviews may have caused not all participants to be equally represented in the results. ${ }^{16}$ However, the focus group moderator (MEDV) is an experienced clinician with more than ten years of clinical experience and ample experience as moderator of group interviews who ensured that all participants were provided the opportunity to express their views. Also, the present study applied within-method triangulation combining data from the written transcripts, videotapes, and the concept mapping approach, which allowed us to derive information from the context outside of the focus group interviews and allowed participants to reflect on their situation beforehand, possibly increasing the information depth during the focus group interviews. ${ }^{15,19}$ Finally, the present study applied purposive sampling to capture EDC needs from different backgrounds and different points in the caregiver career. Purposive sampling is often used in qualitative research and has been used and justified in previous research on caregiver needs. ${ }^{12}$ Although random sampling helps to ensure that any unknown influences are distributed equally amongst groups and can reduce researcher bias in the selection of participants, it can also lead to a sample that is not representative for the measured phenomenon. ${ }^{15}$ As we wanted to ensure a broad sample in terms of background, care recipient stage of the disease and institution, the current approach fitted the research question.

\section{Conclusion}

This study showed that difficulties with acknowledging needs in the early stages can be attributed to a struggle with accepting and adapting to the new role caregivers 
need to take on in the relationship with the care recipient. This underlines the importance of early stage interventions aimed at the facilitation of the adaptation process. ${ }^{27}$ But early stage interventions may do more harm than good if they do not fit the situation and are stigmatizing for the caregiver and care recipient. ${ }^{6}$ Currently available information and support for caregivers is often of a negative, stigmatizing character, which makes it difficult for early-stage dementia caregivers (EDC) to identify with and may frustrate acceptance. Early therapeutic interventions should not be problem-oriented or stigmatizing, but should help caregivers identify their needs based on their individual situation, increase their knowledge on changes in roles and focus more on the enhancement of the positive, intact experiences. With that focus in mind, early therapeutic interventions may strengthen the relationship and reduce negative consequences of caregiving at later stages. ${ }^{6,8,26,27}$ 


\section{References}

1. Alzheimer Disease International. World Alzheimer Report. London: ADI, 2011.

2. Macdonald A, Cooper B. Long-term care and dementia services: an impending crisis. Age Ageing 2007; 36(1): 16-22.

3. de Vugt ME, Stevens F, Aalten P, et al. Do caregiver management strategies influence patient behaviour in dementia? Int J Geriatr Psychiatry 2004; 19(1): 85-92.

4. Blieszner R, Roberto KA. Care partner responses to the onset of mild cognitive impairment. Gerontologist 2010; 50(1): 11-22.

5. De Lepeleire J, Wind AW, lliffe $S$, et al. The primary care diagnosis of dementia in Europe: an analysis using multidisciplinary, multinational expert groups. Aging Ment Health 2008; 12(5): 568-76.

6. de Vugt ME, Verhey FR. The impact of early dementia diagnosis and intervention on informal caregivers. Prog Neurobiol 2013; 110: 54-62.

7. Gaugler JE, Kane RL, Kane RA, Newcomer R. Early community-based service utilization and its effects on institutionalization in dementia caregiving. Gerontologist 2005; 45(2): 177-85.

8. Kjallman Alm A, Hellzen O, Norbergh KG. Experiences of long term ongoing structured support in early stage of dementia - a case study. Int $\mathrm{J}$ Older People Nurs 2013.

9. Verhey $F$, de Vugt $M$. Mild cognitive impairment is becoming more psychosocial. Aging Ment Health 2013; 17(3): 265-6.

10. Brodaty $\mathrm{H}$, Thomson $\mathrm{C}$, Thompson $\mathrm{C}$, Fine M. Why caregivers of people with dementia and memory loss don't use services. Int J Geriatr Psychiatry 2005; 20(6): 537-46.

11. Wolfs $C A$, de Vugt ME, Verkaaik M, et al. Rational decision-making about treatment and care in dementia: A contradiction in terms? Patient Educ Couns 2011.

12. Vaingankar JA, Subramaniam M, Picco L, et al. Perceived unmet needs of informal caregivers of people with dementia in Singapore. Int Psychogeriatr 2013; 25(10): 1605-19.

13. Afram B, Verbeek $H$, Bleijlevens $M H$, Hamers JP. Needs of informal caregivers during transition from home towards institutional care in dementia: a systematic review of qualitative studies. Int Psychogeriatr 2014: 1-12. 
14. Phung KT, Waldorff FB, Buss DV, et al. A three-year follow-up on the efficacy of psychosocial interventions for patients with mild dementia and their caregivers: the multicentre, rater-blinded, randomised Danish Alzheimer Intervention Study (DAISY). BMJ Open 2013; 3(11).

15. Patton MQ. Qualitative Research \& Evaluation Methods. Thousand Oaks London New Delhi: SAGE Publications; 2002.

16. Hennink MM. International focus group research. A handbook for the health and social sciences. Cambridge: University Press; 2007.

17. Eisma R, Dickinson A, Goodman J, Syme A, Tiwari L, Newell AF. Early user involvement in the development of information technology-related products for older people. Universal Access in the Information Society 2004; 3: 131-40.

18. Sleeswijk Visser F, Stappers PJ, van der Lugt R. Contextmapping: experiences from practice. CoDesign: International Journal of CoCreation in Design and the Arts 2005; 1(2).

19. van Rijn $\mathrm{H}$, van Hoof J, Stappers PJ. Designing leasure products for people with dementia: developing "the Chitchatters" game. Am J Alzheimers Dis Other Demen 2010; 25(1): 74-89.

20. Elo $\mathrm{S}$, Kyngas $\mathrm{H}$. The qualitative content analysis process. $J$ Adv Nurs 2008; 62(1): 107-15.

21. Hsieh HF, Shannon SE. Three approaches to qualitative content analysis. Qual Health Res 2005; 15(9): 1277-88.

22. Morgan DG, Walls-Ingram S, Cammer A, et al. Informal caregivers' hopes and expectations of a referral to a memory clinic. Soc Sci Med 2014; 102: 111-8.

23. Wolfs CA, Kessels A, Severens JL, et al. Predictive factors for the objective burden of informal care in people with dementia: a systematic review. Alzheimer Dis Assoc Disord 2012; 26(3): 197-204.

24. Donaldson C, Tarrier N, Burns A. Determinants of carer stress in Alzheimer's disease. Int J Geriatr Psychiatry 1998; 13(4): 248-56.

25. Austrom MG, Lu YY, Perkins AJ, Boustani M, Callahan CM, Hendrie HC. Impact of Noncaregiving-Related Stressors on Informal Caregiver Outcomes. Am J Alzheimers Dis Other Demen 2014.

26. Shim B, Barroso J, Davis LL. A comparative qualitative analysis of stories of spousal caregivers of people with dementia: negative, ambivalent, and positive experiences. Int J Nurs Stud 2012; 49(2): 220-9. 
27. Banningh LW, Vernooij-Dassen MJ, Vullings M, Prins JB, Rikkert MG, Kessels RP. Learning to live with a loved one with mild cognitive impairment: effectiveness of a waiting list controlled trial of a group intervention on significant others' sense of competence and well-being. Am J Alzheimers Dis Other Demen 2013; 28(3): 228-38.

28. Stroebe $\mathrm{M}$, Schut $\mathrm{H}$. The dual process model of coping with bereavement: a decade on. Omega (Westport) 2010; 61(4): 273-89.

29. de Vugt ME, Stevens F, Aalten $P$, et al. Behavioural disturbances in dementia patients and quality of the marital relationship. Int $J$ Geriatr Psychiatry 2003; 18(2): 149-54.

30. Denzin NK. The Research Act. New York: Prentice Hall; 1989. 



\section{CHAPTER 5}

Development and initial evaluation of the web-based selfmanagement program 'Partner in Balance' for family caregivers of people with early-stage dementia: an exploratory mixedmethods study

JMIR Research Protocols, 2016

Lizzy MM Boots, Marjolein E de Vugt, Hanneke EJ Withagen, Gertrudis IJM Kempen \& Frans RJ Verhey 


\section{ABSTRACT}

Background: People with dementia increasingly depend on informal caregivers. Internet-based self-management interventions hold considerable promise for meeting the educational and support needs of early-stage dementia caregivers (EDC) at a reduced cost.

Objective: This study aimed (a) to develop an online self-management program for EDC to increase self-efficacy and goal attainment, and (b) to evaluate the program's feasibility and report preliminary data on effectiveness.

Methods: Based on the Medical Research Counsel (MRC) framework for the development and evaluation of complex interventions, a stepwise approach was adopted to explore potential user needs and develop and validate the content by means of (1) focus group discussions with dementia caregivers ( $N=28)$, (2) interviews with dementia care professionals $(\mathrm{N}=11)$, and (3) individual think aloud usability tests with EDC $(\mathrm{N}=2)$ and experts $(\mathrm{N}=2)$. A pilot evaluation was conducted with EDC ( $\mathrm{N}=17)$ to test the feasibility and establish preliminary effects. Self-report measures of feasibility were completed at post-intervention. Self-efficacy and goal attainment were completed pre- and post-intervention.

Results: The different steps provided useful information about the needs of potential users regarding the content and delivery of the program. This resulted in the newly developed 'Partner in Balance' program. At the start, system failures resulted in a high non-completer rate $(41.2 \%)$, but at the end, a good feasibility score of 209 (range 54-234) was found. The convenience of completing the program at home, the tailored content and the guidance (face-to-face and online) were appraised positively. Preliminary effects on caregiver self-efficacy $(P<0.05)$ and goal attainment $(T>50)$ were promising.

Conclusions: Adaptations were made to the program to limit the amount of system failures and prevent high non-completer rates. As recommended by the MRC framework, confirming the feasibility and preliminary effectiveness is a valuable step toward examining the effectiveness of this newly developed intervention. 


\section{INTRODUCTION}

Nowadays, chronic illness and decreased wellbeing are expected to become global public health challenges, ${ }^{1}$ with dementia being one of the most common disorders in elderly. ${ }^{2}$ With less formal healthcare available and more people in need of care, the caring role has shifted to the informal caregivers at home. ${ }^{3}$ However, dementia caregivers are at increased risk for burden, stress, and have a fourfold risk of becoming depressed compared to non-caregivers. ${ }^{4,5}$ As such, this transition into the caring role increases the need for effective caregiver interventions to improve their mood and quality of life.

Although recent face-to-face caregiver interventions appeared to be promising, ${ }^{6,7}$ the increasing gap between care supply and demand calls for alternative and cheaper methods for providing education and support to informal caregivers. $^{3,8}$ Internet interventions may help caregivers cope with the challenges of caring for a person with dementia. ${ }^{9} \mathrm{~A}$ recent literature review showed that currently available Internet interventions for dementia caregivers have promising effects on caregiver confidence and burden, given that they comprised of multiple components and were tailored to the individual participant. ${ }^{10}$ In addition, internet-delivered caregiver support may prevent accessibility problems for informal caregivers who are isolated or have difficulties accessing traditional healthcare services. ${ }^{11,12}$

At present, remote support for dementia caregivers is increasing, and new Internet interventions are being developed. ${ }^{13-19}$ These programs are, however, mainly focused at dealing with dementia-related problems (e.g. neuropsychiatric symptoms) that occur at an advanced stage of the caregiver career, whereas early-stage interventions can prepare caregivers for their future tasks at a stage where stress and burden are relatively low. ${ }^{20}$ Early intervention and support for caregivers has proven to be effective in reducing strain, increasing caregiver confidence, and delaying institutionalization of the person with dementia. ${ }^{21-23}$ Moreover, early therapeutic interventions may help caregivers identify their needs based on their individual situation and facilitate the adaptation process. ${ }^{24}$ The Stress and Coping paradigm by Lazarus and Folkman ${ }^{25}$ and the Social Learning theory by Bandura ${ }^{26}$ propose that taking charge of the changes in one's life increases self-efficacy, and can therefore reduce caregiver stress and its impact on general wellbeing. ${ }^{27}$ Following these theories, an early-stage support program for caregivers should focus at positively managing life with dementia rather than managing the dementia itself. ${ }^{28}$ Self-management programs suit the caring role transition and have previously been 
used to support informal caregivers of several chronic diseases with promising results. ${ }^{12,29,30}$

\section{Iterative development process}

The present study describes the development of an online self-management program for EDC to improve self-efficacy and goal attainment. We closely followed the iterative process of the new Medical Research Counsel (MRC) Framework for the development of complex interventions. ${ }^{31}$ The first two steps in the intervention development are described elsewhere. ${ }^{10,24}$ The current paper describes the next four steps spread over a 2-year time period (2012-2014) (See Figure 1). These steps are described below.

\begin{tabular}{|c|c|c|c|c|}
\hline Former Steps & Step 1 & Step 2 & Step 3 & Step 4 \\
\hline $\begin{array}{l}\text { 1. Literature } \\
\text { review } \\
\text { 2. Explore } \\
\text { early-stage } \\
\text { caregiver } \\
\text { needs }\end{array}$ & $\begin{array}{c}\text { Explore } \\
\text { potential user } \\
\text { views }\end{array}$ & $\begin{array}{l}\text { Develop and } \\
\text { validate the } \\
\text { content and } \\
\text { structure of } \\
\text { the online }\end{array}$ & $\begin{array}{c}\text { Test the } \\
\text { feasibility and } \\
\text { preliminary } \\
\text { effectiveness }\end{array}$ & $\begin{array}{l}\text { Adapt the } \\
\text { program } \\
\text { based on } \\
\text { feasibility } \\
\text { findings }\end{array}$ \\
\hline
\end{tabular}

Figure 1. Iterative development process informed by MRC framework

\section{Step 1: Explore potential user views: focus groups METHODS}

In-depth exploratory focus group interviews were used to explore EDC views on the content and format of an early-stage intervention (See $\operatorname{Ref}^{24}$ for detailed methodology). A context-mapping approach ${ }^{32}$ was used; a booklet examining personal Internet- and computer use and a collage displaying chosen themes based on significance during the early stages. ${ }^{32}$ Available themes were preselected based on existing interventions, early-stage dementia care literature, ${ }^{21,33}$ and expert knowledge. Blank cards were also provided. The most often selected and highest appraised themes were compiled. Focus group interviews were transcribed verbatim and analyzed independently based on deductive content analysis by authors LMMB and MEdV. Topics that were mentioned frequently and explicitly served as the basis for the categorization. Categories were merged into common themes in a consensus meeting (LMMB and MEdV). 


\section{RESULTS}

Participant characteristics $(\mathrm{N}=28)$ are displayed in Table 1. The booklet on computer use was completed by 18 participants. Reasons for non-completion were (1) overlooking the booklet $(\mathrm{N}=7)$, and (2) not understanding its value ( $\mathrm{N}=3)$. Participants used the computer for multiple purposes, e.g., finding information (83\%), e-mail $(78 \%)$, financial transactions $(78 \%)$, writing $(78 \%)$, viewing photos $(50 \%)$, playing games (44\%), (video) chatting with family members (17\%), and shopping $(11 \%)$. Three participants did not use a computer and were not inclined to do so in the near future. During the focus groups, the majority of the participants considered Internet interventions as efficient due to the high level of accessibility, especially when feeling pressed for time or being bound to one's home. Receiving answers to urgent queries was also considered very positive.

"The advantages are no travelling time and the possibility to search what I want to know when it is convenient for me." (P9)

"I would like to be able to extract the information that is important for me at that particular moment, because we're all so different. When you're in need of an answer, a personal response would be great." (P13)

Blended care (face-to-face care combined with online modules) was preferred over online care only, due to the personal contact with a professional.

"People experience emotions, while a computer is just an object. Seeing the person you are talking to is really important. Once you know each other, email or telephone is fine for information exchange." (P5)

All participants $(\mathrm{N}=28)$ completed the collage of themes during the interviews. Participants stressed the importance of an intervention tailored to the stage of the disease and the individual caregiver's situation, with less focus on coping with dementia and negative stigmatizing information about the future. Learning how to stay healthy by positively managing one's life and learning to accept the changes were considered important, as well as the significance of information provided by other caregivers. A more flexible choice of themes, based on personal needs and areas of interest, was considered desirable. The themes most often mentioned and 
highest appraised are listed in Table 2.

Table 1. Background characteristics of the caregivers $(\mathrm{N}=28)$ and the care recipients $(\mathrm{N}=25)$

\begin{tabular}{|c|c|c|}
\hline Age (mean) & 63.6 years & \\
\hline \multirow[t]{2}{*}{ Gender } & Men & $7(25 \%)$ \\
\hline & Women & $21(75 \%)$ \\
\hline \multirow[t]{5}{*}{ Relationship to the care recipient } & Spouse & $22(78 \%)$ \\
\hline & Child & $2(7 \%)$ \\
\hline & Child-in-law & $2(7 \%)$ \\
\hline & Sibling & $1(4 \%)$ \\
\hline & Friend & $1(4 \%)$ \\
\hline \multirow[t]{2}{*}{ Living together with care recipient } & Yes & $21(75 \%)$ \\
\hline & No & $7(25 \%)$ \\
\hline \multirow[t]{6}{*}{ Care recipient diagnosis } & $\mathrm{MCl}$ due to $\mathrm{AD}$ & $8(32 \%)$ \\
\hline & $A D$ & $11(44 \%)$ \\
\hline & Vascular & $3(12 \%)$ \\
\hline & Dementia & \\
\hline & Parkinson & $1(4 \%)$ \\
\hline & Dementia NOS ${ }^{a}$ & $2(8 \%)$ \\
\hline \multirow[t]{4}{*}{ Care recipient years of diagnosis } & 0.5 & $5(20 \%)$ \\
\hline & $1-3$ & $9(36 \%)$ \\
\hline & $4-6$ & $10(40 \%)$ \\
\hline & $7-10$ & $1(4 \%)$ \\
\hline
\end{tabular}

${ }^{\mathrm{a}} \mathrm{NOS}=$ Not Otherwise Specified

Table 2. Themes most often selected and highest appraised by family caregivers $(\mathrm{N}=28)$

\begin{tabular}{llll}
\hline Themes & $\begin{array}{l}\text { Selected, } \\
(\%)\end{array}$ & $\begin{array}{l}\mathrm{n} \\
\text { Appraised } \\
\text { important, } \mathrm{n}(\%)\end{array}$ & most \\
\hline Practical tips & $27(96 \%)$ & $14(50 \%)$ \\
Role and relationship changes & $22(79 \%)$ & $5(18 \%)$ \\
Information about the disease & $21(75 \%)$ & $12(43 \%)$ \\
Balance in activities & $19(68 \%)$ & $2(7 \%)$ \\
Focus on the positive & $19(68 \%)$ & $5(18 \%)$ \\
Communication & $18(64 \%)$ & $5(18 \%)$ \\
Acceptance & $16(57 \%)$ & $9(32 \%)$ \\
Insecurities and worrying & $12(43 \%)$ & $3(11 \%)$ \\
Social relationships and support & $12(43 \%)$ & $1(4 \%)$ \\
Emotions and tension & $10(36 \%)$ & $1(4 \%)$
\end{tabular}




\section{Step 2: Develop and validate program content and structure Consulting dementia care experts METHODS}

Individual in-depth interviews were conducted to explore dementia care experts' views on EDC Internet support. Experts from different institutions and regions within the Netherlands were recruited via email. Inclusion criteria were (1) professional caregiver in the Dutch dementia care field, (2) daily interaction with people with dementia (PwD) and their caregivers, and (3) ample experience with supporting EDC. The number of participants $(\mathrm{N}=11)$ was determined by data saturation; the redundancy of themes emerging from interviews [34]. Professional backgrounds consisted of psychiatrists $(\mathrm{N}=1)$, clinical neuropsychologists $(\mathrm{N}=3)$, registered health psychologists $(\mathrm{N}=4)$, occupational therapists $(\mathrm{N}=1)$, social psychiatric nurses $(\mathrm{N}=1)$, and nurse practitioners ( $N=1)$, with an average of 13.64 (SD 7.43) years of professional experience. A semi-structured interview guide was developed by authors LMMB and MEdV, validated by author FRJV. Topics included EDC needs, relevance and feasibility of EDC support, and themes for an EDC intervention. Brief summaries of the key points were made throughout the interview to obtain participant verification. $^{35}$ All interviews were audiotaped. The content of the verbatim transcriptions of the interviews was analyzed by summarizing common themes based on deductive content analysis.

\section{RESULTS}

The experts emphasized that caregiver support needs to be tailored to the dementia stage. Concerns were raised about providing early support in the absence of laterstage problems, when caregivers are not in need of help yet and could possibly reject early-stage support. Experts considered education on the disease and its course as most important. Other important themes involved accepting the disease, coping with relationship changes, stress, role management, and rumination. The importance of interaction between PwD, caregivers and environment was also stressed. Correcting or accepting care recipients' mistakes and notifying social network can be primary stressors in daily interaction. Too much negative information not fitting the early stages, e.g., behavioral problems, care homes and end of life decisions, could lead to adverse reactions and should be avoided in EDC support. 
Combining the themes chosen by the experts and the caregivers resulted in 9 separate themes (Table 3). The theme 'practical tips' was incorporated in the module structure to provide tips thematically.

Table 3. Modules of 'Partner in Balance' and their key points

\begin{tabular}{|c|c|}
\hline Module & Key points \\
\hline Acceptance & $\begin{array}{l}\text { - } \quad \text { Identify changeable and unchangeable situations } \\
\text { - } \quad \text { Adapt expectations and learn to let go }\end{array}$ \\
\hline Balance in activities & $\begin{array}{ll}\text { - } & \text { Change in daily and pleasant activities } \\
\text { - } & \text { Identify personal carrying capacity and burden }\end{array}$ \\
\hline $\begin{array}{l}\text { Communication with } \\
\text { family member and } \\
\text { environment }\end{array}$ & $\begin{array}{l}\text { - } \quad \text { Communication changes due to memory problems } \\
\text { - } \quad \text { Effective communication with adaptations }\end{array}$ \\
\hline Coping with stress & $\begin{array}{ll}\text { - } & \text { Relationship between stress and health problems } \\
\text { - } & \text { Identify and cope with stress in daily life }\end{array}$ \\
\hline Focusing on the positive & $\begin{array}{ll}\text { - } & \text { Identify activities and situations that are still possible } \\
\text { - } & \text { Find alternatives and accept adaptations }\end{array}$ \\
\hline $\begin{array}{l}\text { Insecurities and } \\
\text { rumination }\end{array}$ & $\begin{array}{ll}\text { - } & \text { Recognize rumination signals and control thoughts } \\
\text { - } & \text { Prevent looking ahead; live in the moment }\end{array}$ \\
\hline Self-understanding & $\begin{array}{ll}\text { - } & \text { Self-evaluation in caregiver encounters } \\
\text { - } & \text { Personal strengths and areas of improvement }\end{array}$ \\
\hline $\begin{array}{l}\text { The changing family } \\
\text { member }\end{array}$ & $\begin{array}{l}\text { - } \quad \text { The changing memory and behavior } \\
\text { - } \quad \text { Influence of memory decline on daily life together }\end{array}$ \\
\hline $\begin{array}{l}\text { Social relations and } \\
\text { support }\end{array}$ & $\begin{array}{l}\text { - } \quad \text { Value and maintenance of social relations } \\
\text { - } \quad \text { Types of support }\end{array}$ \\
\hline
\end{tabular}

\section{Content proposal: evidence and conceptual frameworks on self-care METHODS}

Intervention content was proposed by authors LMMB and MEdV based on a literature review, ${ }^{10}$ EDC needs, ${ }^{24}$ identified themes in Step 1, and conceptual frameworks on self-management. The Stress and Coping paradigm ${ }^{25}$ served as the theoretical basis for the content of the modules. According to this model, stress is experienced when a person perceives that the demands (caring for a person with dementia) exceed their personal and social resources. Caregivers' responses to their stress situation might be mediated by their understanding of the situation and their beliefs about their ability to cope. The latter fits Bandura's ${ }^{26}$ concept of self-efficacy (belief in one's capabilities). Consistent with this theory, models of dementia management 
emphasize the need to maintain self-worth and control. ${ }^{28}$ An intervention aimed at increasing self-efficacy should not only educate the caregiver, but should foster selfmanagement by combining education with problem-solving skills, and work towards a change in behavior. ${ }^{36}$

\section{RESULTS}

The proposed self-management intervention program 'Partner in Balance' encourages caregivers to actively manage their lives and identify solutions for their specific needs. ${ }^{37}$ Increasing knowledge, identifying and setting goals, and learning skills to achieve these previously set goals served as the basis for the intervention program. Module content was focused on role management (e.g., balancing activities in daily life) and emotional management (e.g., dealing with fear and insecurity about the future). ${ }^{38}$ Formulating, planning and executing personal goals can be learned using a pro-active 5-step change plan (Textbox 1) often used in self-management, ${ }^{38}$ which was integrated into each module. By formulating and planning a personal change plan, caregivers learn to anticipate on stressful situations and gain confidence in their ability to take care of the situation and themselves. ${ }^{38}$ Because caregivers greatly varied in their needs, personal goals and interest, a flexible choice of modules was used. Successful elements that were identified in the literature review, ${ }^{10}$ including tailored caregiving strategies and contact with a coach and/or other caregivers, were included in the program content likewise.

Textbox 1. The self-management 5-steps applied per module

\section{Steps to create an action plan and obtain goals}

Step 1: Recognize areas that you wish to change or to maintain

Step 2: Recognize additional conditions and barriers

Step 3: Generate alternative strategies for the problem(s)

Step 4: Write down your final plan SMART

(Specific, Measurable, Attainable, Realistic and Timely)

Step 5: Evaluate when you will be satisfied with your progress

\section{Validating proposed program content METHODS}

Dementia care experts $(\mathrm{N}=4)$ not involved in the initial interviews were asked to read the material and provide comments with respect to language, tone, amount of 
information and significance and propriety of the content. They worked as clinical neuropsychologist $(\mathrm{N}=1)$, registered health psychologist $(\mathrm{N}=1)$, occupational therapist $(\mathrm{N}=1)$, and social psychiatric nurse $(\mathrm{N}=1)$, with an average of 9.75 years (SD 2.22) years of professional experience.

\section{RESULTS}

The experts provided comments for the content of each module. Stigmatizing, complex, or unclear language was reported and alternatives were provided. The textual content of the program was adapted accordingly.

\section{Step 3: Testing the feasibility and preliminary effectiveness Think Aloud Usability Testing METHODS}

A web-based fully operational program was developed based on the senior-friendly website checklist. ${ }^{39}$ Initial usability flaws were tested with the 'think aloud' method. ${ }^{40}$ Potential users were asked to think aloud while using the system, allowing the researchers to understand the reasons behind their usage behavior. Participants $(\mathrm{N}=4)$ were randomly selected from the focus group interviews $(\mathrm{N}=2)$ and the expert interviews $(\mathrm{N}=2)$. All aspects of the senior-friendly website guidelines were checked (e.g., font size, contrast, menus and navigation, button style and size, phrasing, illustrations and videos, and web-assistance) and additional comments on userfriendliness were explored in depth. The interviews were audiotaped and the verbatim transcripts were combined with field notes made by author LMMB on experienced difficulties during the walk-through. A coding scheme based on the interview protocol was used. ${ }^{40}$

\section{RESULTS}

All participants $(\mathrm{N}=4)$ commented on the lay-out, font size, contrast, tone, and navigation. The website lay-out was considered professional and attractive, although a uniform composition of all pages was proposed to foster cohesion. Alterable font sizes and increased contrast with the background color were suggested. Vignettes of caregivers were considered useful, but addressing people with their given name instead of their family name was considered more appealing. The tips from other caregivers were perceived as crucial. It was suggested to conclude every module with these tips to increase lay-out uniformity. 


\section{Piloting feasibility METHODS}

An uncontrolled pre-post-intervention pilot study with EDC was conducted to establish feasibility, as this is recommended before moving on to a larger scale effect study. ${ }^{31,41}$ The Medical Ethics Committee of the Maastricht University Medical Centre (MUMC+) approved this study (\#NL44475.068.13, Dutch trial register: \#NTR4217). Caregivers were included in the study if they (1) were spousal caregivers of people with Mild Cognitive Impairment $(\mathrm{MCl})^{42}$ or mild dementia of all subtypes, ${ }^{43}$ and (2) had access to the Internet. Exclusion criteria were (1) insufficient cognitive abilities to engage in the online self-management program, (2) overburdening, (3) severe health problems (determined by study staff), or (4) caring for PwD caused by human immunodeficiency virus, acquired brain impairment, Down syndrome, Huntington's chorea, or alcohol abuse. Participants were recruited at memory clinics and ambulatory mental health clinics. Based on comparable feasibility studies, we aimed to include 10 participants. ${ }^{44,45}$ Of those contacted, 17 out of 43 caregivers (40\%) were willing to participate and signed the informed consent.

Feasibility was evaluated face-to-face at the caregiver's home by a semistructured interview developed for this study, the Program Participation Questionnaire (PPQ). The PPQ was based on measurement scales for perceived usefulness and ease of use and overall acceptance of information technology $y^{46,47}$ and included 30 items on usability, clarity, comfort with and acceptability of the format on a 7-point Likert scale ranging from 1 (strongly disagree) to 7 (strongly agree). For the individual PPQ items, see Multimedia Appendix 1. Mean scores were calculated with descriptive statistics. As there were no external criteria to properly define feasibility, ${ }^{48}$ we followed the conventional strategy of using the median score of the questionnaire as a cut-off. This approach was previously adopted in a Delphi study as evidence of agreement of intervention feasibility. ${ }^{49}$ Based on the PPQ scale (range 54-234, median 144), scores of 145 or higher were considered as acceptable feasibility. Mean item scores (range 1-7) will be used to make decisions on positively and negatively appraised aspects of the program. Mean item scores of 5 (slightly agree) or higher will be considered positive, mean item score below 4 (slightly disagree or lower) will need further revisions. Participants were asked to elaborate their scores. Comments were audio recorded and transcribed verbatim. Meaningful data units based on the PPQ items were identified and derived independently from the qualitative data by authors LMMB and HEJW with deductive content analysis. 
Furthermore, the actual accessed data use of the program (number of log-ins and features used) was compared to self-report data.

\section{RESULTS}

The study population consisted of 17 participants, of whom ten completed the postintervention assessment. Participants who did not complete the post-intervention assessment were replaced to still meet the sample size suggested by previous studies $[44,45]$. The main reasons for not completing the program and the postintervention assessment were difficulties with the online aspect of the program $(\mathrm{N}=4)$, private circumstances $(\mathrm{N}=2)$, and disagreements with the care recipient $(\mathrm{N}=1)$. Completer and non-completer characteristics are listed in Table 3.

The PPQ showed a good internal consistency $(\alpha=0.89)$ and a mean sum score of 209 (SD 22.14). Given the threshold of 145 or higher, this score indicated an acceptable feasibility. Mean item scores above 5 (slightly agree or higher) were found for convenience of completing the program at home $(5.9, S D 1.8)$, clarity of the website (6.0, SD 1.2), module structure (6.1, SD 0.6) and content (6.6, SD 0.5), privacy (6.6, SD 1.3), tailored assignments (6.1, SD 1.3), guidance by the coach (6.6, SD 0.5), and general contentment (6.4, SD 0.9). A mean item score below 4 (slightly disagree or lower) was found for usefulness of the discussion forum (2.8, SD 2.7). Table 4 shows the positive, negative and neutral themes derived from the additional comments. Self-report usage data were comparable to tracked usage data; 106.41 (SD 96.15) minutes spent per module, including scoping the website (4.4 minutes, SD 4.13), completing the assignments and change plan (79.14 minutes, SD 77.16), contacting the personal coach (15.31, SD 16.96) and visiting the discussion forum, spread out over 2.38 (SD 1.38) weeks.

\section{Piloting preliminary effects METHODS}

Preliminary understanding of the effectiveness of the program was based on the baseline- and post-intervention assessment 8 weeks later, completed at the participant's own convenience in an uncontrolled pilot study. At participant request, paper questionnaires were used. The Caregiver Self-Efficacy Scale (CSES) was used to measure domain-specific caregiver self-efficacy. ${ }^{50}$ The subscales include four items on service use and six items on care management, with scores ranging from 1 (not at all certain) to 10 (very certain). We found a good internal consistency 
Table 3. Participant characteristics for completers and non-completers of the intervention

\begin{tabular}{lllll}
\hline & \multicolumn{2}{l}{ Completers $(N=10)$} & \multicolumn{2}{l}{ Non-completers $(N=7)$} \\
\cline { 2 - 5 } & Mean & $S D$ & Mean & $S D$ \\
\hline Age caregiver & 68.10 & 6.54 & 67.43 & 5.65 \\
\hline Age care recipient $(P w D)$ & 69.90 & 4.33 & 71.57 & 8.46 \\
\hline Hours care per week & 44.20 & 56.85 & 76.43 & 71.98 \\
\hline Gender $(N, \%)$ & & & $2(12 \%)$ & \\
Male & $7(41 \%)$ & & $5(29 \%)$ & \\
Female & $3(18 \%)$ & & & \\
\hline Education $(N, \%)$ & & $3(18 \%)$ & \\
High school & $1(6 \%)$ & & $4(23 \%)$ & \\
College & $7(41 \%)$ & & $0(0 \%)$ & \\
Graduate school & $2(12 \%)$ & & & \\
\hline PwD diagnosis $(N, \%)$ & $7(41 \%)$ & & $5(29 \%)$ & \\
MCl & $3(18 \%)$ & & $1(6 \%)$ & \\
Alzheimer dementia & $0(0 \%)$ & & \\
Vascular dementia & & & \\
\hline anon-completer rate $=41.2 \%$ & & & & \\
\end{tabular}

${ }^{a}$ non-completer rate $=41.2 \%$

for both service use $(\alpha=0.73)$ and care management $(\alpha=0.87)$. Paired samples t-tests were conducted to evaluate pre-post intervention changes. The Goal Attainment Scaling (GAS) ${ }^{51}$ method was used to rate treatment-related change and to compare relative success of previously set personal goals. Baseline scores were set at -2 . Post-intervention scores can range from -2 (much lower than expected) to +2 (much better than expected), with a score of 0 meaning goal attained. Raw scores were transformed into an individual mean GAS score (T-score) to determine goal attainment with a potential weight assigned to the goal(s). ${ }^{52} \mathrm{~T}$-scores of $\geq 50$ (SD 10) indicate effective goal achievement.

\section{RESULTS}

Post-intervention, participants $(\mathrm{N}=10)$ had significantly higher scores on both the CSES care management subscale $(M=41.1, S E=2.5 ; \mathrm{t}(9)=-2.5, P=.03)$ and service use subscale $(\mathrm{M}=32.6, \mathrm{SE}=1.7 ; \mathrm{t}(9)=-3.5, P=.01)$ compared to pre-intervention $(M=36.1, S E=3.2$ and $M=23.2, S E=3.4$, respectively), although effect sizes were small ( $d=0.14$ and $d=0.41$, respectively). 


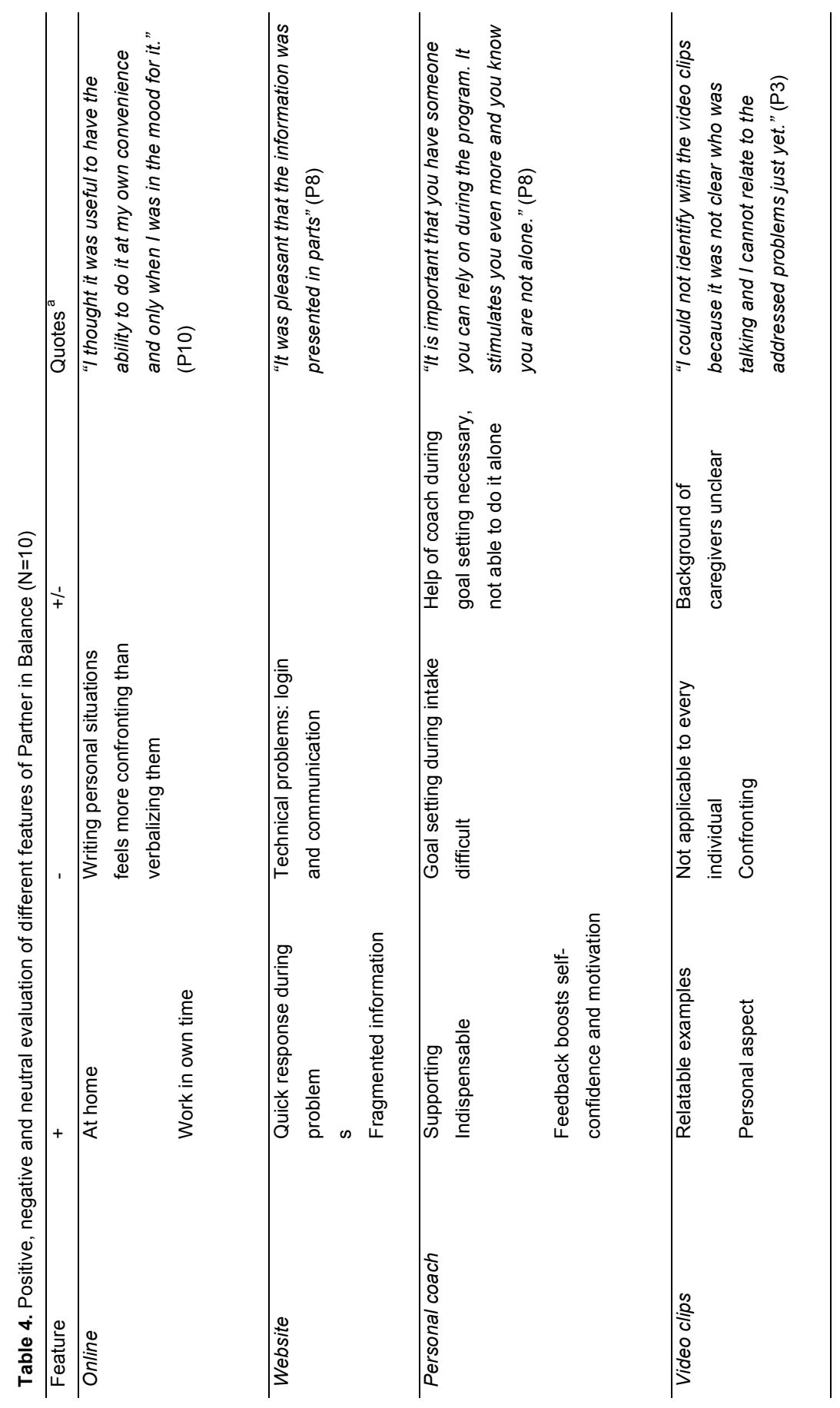




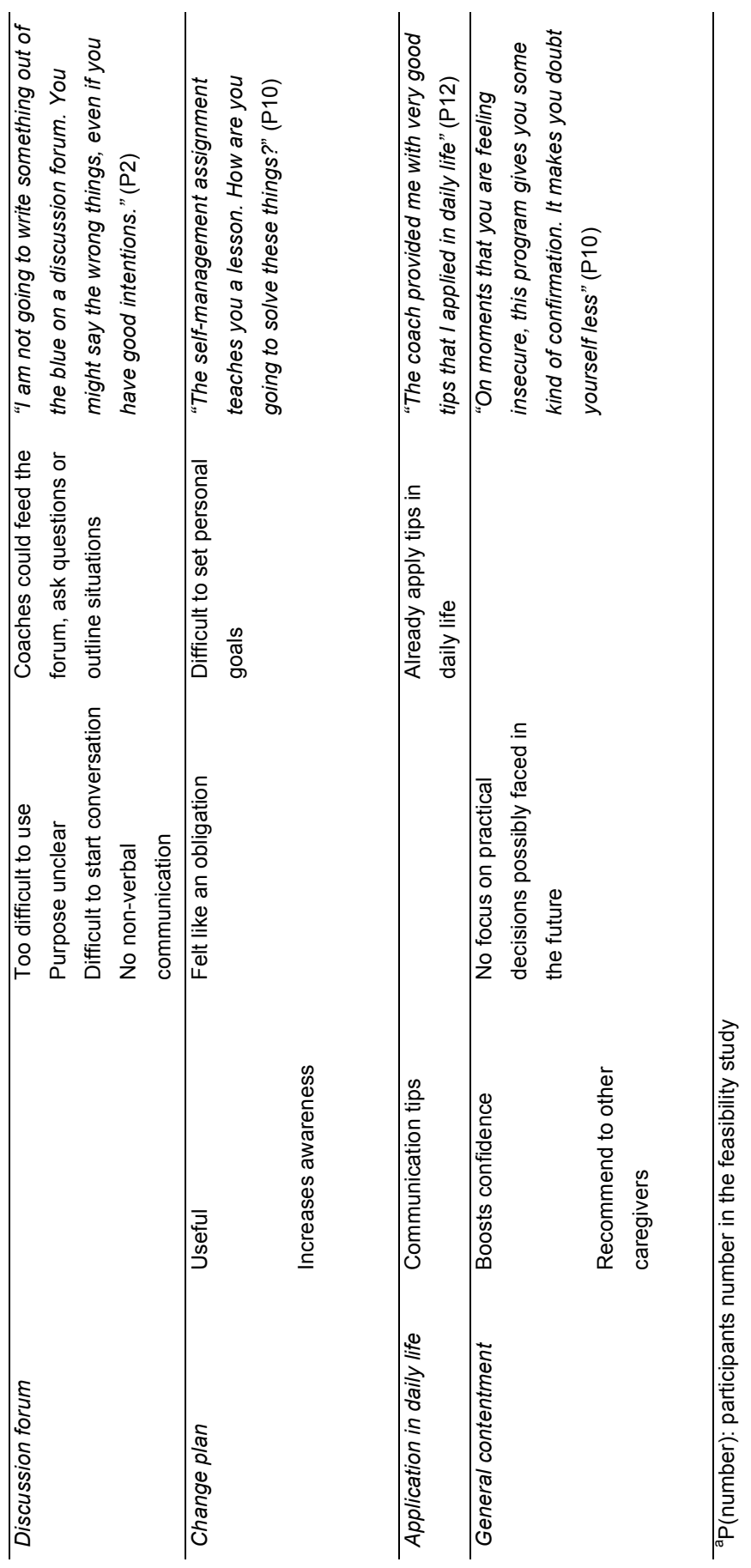


Eight program completers set 13 goals in total. Two program completers were not able to set goals due to personal difficulties verbalizing the desired change. Two goals (of 2 participants with multiple goals) could not be scored post-intervention, because the goals changed during the course of the study. Eight goals were attained (2 attained, 5 higher than expected, 1 much higher than expected), and 3 goals were not attained (1 much lower than expected, 1 lower than expected). The mean T-score at baseline (set at -2 level) was 27.8 (SD 3.04). The mean achieved T-score at postintervention was 53.7 (SD 12.03). Table 5 shows the number of goals for each domain, with most goals set on communication with the care recipient, followed by maintaining positive activities together, obtaining social support and planning time alone.

Table 5. Number of set goals, attainment scores and domains in which goals were set $(\mathrm{N}=8)$

\begin{tabular}{lllc}
\hline & Mean & $S D$ & Range \\
\hline Number of set goals per participant & 1.6 & 1.06 & $1.0-4.0$ \\
\hline GAS $^{a}$ score at baseline & 27.8 & 3.04 & $22.6-30.0$ \\
\hline GAS $^{a}$ score achieved & 53.7 & 12.03 & $30.0-70.0$ \\
\hline Number of set goals per domain $(N)$ & & & \\
- Communication with care recipient & 7 & & \\
- Activities with care recipient & 2 & & \\
- Social support & 2 & & \\
- Time alone & 2 & & \\
\hline${ }^{a} G A S=$ Goal Attainment Scaling & &
\end{tabular}

\section{Step 4: Adapting the program: final intervention}

Following the iterative development process, the program was adapted according to the results obtained in the feasibility study. The discussion forum was expanded with regular posts from personal coaches with practical tips, literature and events related to EDC. The role and background of the person in the video clips was clarified. Additionally, the content of often-mentioned early-stage situations and problems ${ }^{24}$ was expanded and later-stage problems were made less prominent in the video clips. Furthermore, technical issues with logging in and communicating with the personal coach were resolved with the team of web experts. 


\section{Final intervention}

Partner in Balance consists of 3 elements: (1) face-to-face intake session with a personal coach, (2) online period guided by the personal coach (psychologist or psychiatric nurse with ample experience with dementia caregivers), and (3) face-toface evaluation session with the personal coach.

\section{Intake session}

In the intake session participants are introduced to the website and the selfmanagement concept of the program. The coach and participant set personal goals by using a motivational interviewing technique frequently used to identify change objectives and enhance intrinsic motivation. ${ }^{53}$ Based on the discussed areas for improvement, participants select 4 out of the 9 modules that were previously identified by experts and caregivers. Participants are provided with personal login codes to access their selected modules and edit their personal information. After the online period, participants will discuss their personal goals and their ability to cope with future difficulties in the evaluation session.

\section{Online period}

During the online period of the intervention, participants follow the chosen modules during an 8-week period. The website consists of a (1) homepage with a short description of the goal of the program, personal login option, contact information of the researcher and the institutional affiliation (Maastricht University), (2) personal page with a link to the chosen modules and a mailbox for exchange with the personal coach, and (3) online forum to interact with other caregivers, moderated by the researcher and personal coaches.

Every module has a fixed design of 4 components; (1) video clip of fellow family caregivers, (2) education, (3) self-reflection assignment, and (4) the 5-step change plan, guided by the personal coach who will provide individualized online feedback after completion of each module and offers assistance when needed. For every module, two weeks are reserved as a starting point. However, participants are allowed to complete the modules at their own pace as informed by the selfmanagement approach. ${ }^{38}$ The first week of a module addresses component 1-4. Participants can send their assignment and 5-stepplan to their coach. The second week of every module is reserved for feedback from the coach, after which participants can adjust their 5-stepplan if necessary. 


\section{Personal coach}

The personal coach is an experienced dementia care professional (psychologist or psychiatric nurse). Coaches will receive a one-day training in self-management techniques and online help before the start of the intervention. They will receive experienced supervision from an experienced professional in psychology and selfmanagement during the course of the intervention period to ensure quality and alignment of the feedback of the coaches according to self-management principle. Coaches are asked to support participants in choosing modules that fit their personal situation, help participants identify feasible goals, offer techniques to achieve goals and provide participants with general constructive feedback on their assignments. Via a personal login code, coaches will be matched with the participants assigned to them.

\section{DISCUSSION}

In this paper the iterative development process of the web-based self-management program 'Partner in Balance' (PiB) for EDC was presented. Use of the MRC framework enabled us to develop an intervention based on existing research, theoretical frameworks, and user and professional input. Including potential users during the design process enabled us to gain unique insights into usage behavior and challenges in order to adapt the technology to the needs of the target audience. A similar design has been successfully used in previous studies. ${ }^{54-56}$

During the exploration phase, caregivers greatly varied in their need for information. Previous self-management studies confirm that personal caregiver needs should be used as a starting point. ${ }^{57}$ Blended care was preferred over online care only, due to the personal contact with a professional. Previous studies support the value of this format as participants highly appreciated the connection with their coach or therapist ${ }^{58}$ and felt more motivated to complete web-based interventions. ${ }^{59}$ On the other hand, adding face-to-face contacts increases the costs of the online program and reducing the number of face-to-face contacts might harm treatment outcomes when online components are not used. ${ }^{60,61}$ However, blending online modules with regular face-to-face therapy can increase the adherence and effectiveness of the treatment. Scientific validation of blended care interventions is warranted for the development and adaptation of future treatments ${ }^{60}$ and can provide important support for the use of blended care interventions rather than online therapy only. The former can be more easily implemented by health services, therapists and 
clients than online therapy, as they can be integrated in existing treatment and care settings. ${ }^{62}$

Our results showed acceptable rates of satisfaction with PiB. Caregivers greatly appreciated the use of online resources due to the convenience of completing the program from their homes, which is in line with previous studies on web-based caregiver interventions. ${ }^{46,63-65}$ Furthermore, $\mathrm{PiB}$ supported the participants in the process of caregiving and boosted their self-confidence, probably due to the combination of support, self-management, and a tailored approach. ${ }^{66,67}$ However, the non-completer rate was high due to initial technical difficulties, which were resolved later on in the study. Unfamiliarity with using the website also caused difficulties among the older age group, resulting in a relatively young sample. A recent study confirmed that younger dementia caregivers were more likely to use the Internet for health-related purposes [68]. However, other research showed that homebound older adults with limited computer skills who receive computer training at the start of an intervention can participate without difficulties. ${ }^{69}$ Also, including more potential users in the thinking aloud procedure could be helpful. ${ }^{40}$

The discussion forum was negatively reviewed and hardly used, due to the unclear purpose, the anonymity of participants and the high threshold for starting a conversation. However, other studies suggest that forums can serve as a valuable addition to share experiences and support ${ }^{39,45,70}$ Adding new tips or developments for caregivers could increase the use of a forum since the aspect of reading posts was already considered useful. ${ }^{70}$ Furthermore, participants reported struggles with goal setting. This could be due to the relatively lower objective burden of EDC, compared to caregivers of people in later stages of the disease. EDC might experience more subjective burden, which is more difficult to translate into specific needs. Problems with accepting and adapting to their new role may also have hindered goal setting. ${ }^{24}$ Therefore, goal setting for EDC should focus on the enhancement of positive overall experiences and facilitation of the personal adaptation process, rather than exclusively aiming for change. This approach may help to reduce or prevent negative consequences of caregiving (e.g., overburdening) at a later stage.$^{24}$

Preliminary effects on caregiver self-efficacy and goal attainment were small, yet positive. This finding is in line with Bandura's theory on self-efficacy, stating that caregivers' objective understanding of the situation and belief in one's capabilities (i.e., the self-efficacy level) can increase if provided with the right tools. ${ }^{26}$ 
Furthermore, this finding is congruent with previous research on online support for dementia caregivers. ${ }^{21,71,72}$

The program in this study was evaluated in a homogenous group of primary caregivers (e.g. spousal caregivers), with specific attention for the spousal relationship. However, the themes may apply to a broader target group, as demonstrated by previous studies. ${ }^{13-19} \mathrm{PiB}$ could potentially be suitable for other primary carers, which should be further investigated in the upcoming effect study using a larger sample.

\section{Limitations}

The small sample size, the lack of a control condition and a possible sampling bias based on caregivers with access to the Internet, make it difficult to generalize the results. However, previous studies adopting a development and feasibility approach have used similar methodology and sample sizes, ${ }^{15,73}$ fitting the purpose of formative research. ${ }^{31}$ Also, users and experts were closely involved in the development of the intervention, and the content and adaptations relied on in-depth participant and expert feedback. Future research should consider inclusion of caregivers in the proposed content validation, to ensure potential user feedback in every step of the development. Furthermore, drawing conclusions from the adopted median cut-off score, which is an arbitrary value, may not be justified. However, in the present study the overall feasibility score was not leading. Mean item scores were used to make program improvements.

The present study used paper questionnaires. Although seniors' use of the Internet is expected to increase over time ${ }^{74}$ dementia caregivers seem to be less active in health-related Internet use compared to the population at large. ${ }^{68}$ The high non-completer rate resulted in missing post-test data from the non-completers as this was collected after the last module. However, reasons for non-completion and characteristics of non-completers were provided, giving insight into their possible motives. Future effectiveness studies should include non-completer data at posttreatment and follow-up and consider using web-based questionnaires, with the advantages of low costs, no missing data or data entry errors and time flexibility. ${ }^{75}$

\section{Conclusion}

Our tailored intervention approach appeared to be feasible for informal EDC and provided them with important support when dealing with the difficulties of caregiving. 
Feasibility results were used to improve the intervention. Confirmation of the feasibility and preliminary effectiveness is a valuable step towards examining the effectiveness of this intervention, as recommended by the MRC framework. ${ }^{31}$ The $\mathrm{PiB}$ course is currently (November 2015) available for caregivers who are interested in participating in the effectiveness study. At the course website (www.partnerinbalans.nl) they can express their interest by emailing the researcher, after which they will receive additional information about the course and the effectiveness study. 


\section{References}

1. World Health Organization. World health statistics. Geneva: Switzerland, 2014.

URL: http://apps.who.int/iris/bitstream/10665/112738/1/9789240692671_eng.pdf?ua $=1$ [accessed 2015-09-15] [WebCite ID 6bZJDOw1a]

2. Alzheimer's Disease International. Policy Brief for Heads of Government: The Global Impact of Dementia 2013-2050. London: ADI, 2013. URL: https://www.alz.co.uk/research/GloballmpactDementia2013.pdf [accessed 2015-09-15] [WebCite ID 6bZlqjsHx]

3. Alzheimer's Association. Alzheimer's disease facts and figures. Alzheimers Dement 2014 Mar;10(2). PMID:24818261

4. Brodaty H, Donkin M. Family caregivers of people with dementia. Dialogues Clin Neurosci 2009 Jun;11(2):217-28. PMID: 19585957

5. Joling KJ, van Hout HP, Schellevis FG, van der Horst HE, Scheltens P, Knol $\mathrm{DL}$, van Marwijk HW. Incidence of depression and anxiety in the spouses of patients with dementia: a naturalistic cohort study of recorded morbidity with a 6-year follow-up. Am J Geriatr Psychiatry 2010 Feb;18(2):146-53. PMID:20104070.

6. Van Mierlo LD, Meiland FJ, Van der Roest HG, Droes RM. Personalised caregiver support: effectiveness of psychosocial interventions in subgroups of caregivers of people with dementia. Int J Geriatr Psychiatry 2012 Jan;27(1):114. PMID:21520288

7. Selwood A, Johnston K, Katona C, Lyketsos C, Livingston G. Systematic review of the effect of psychological interventions on family caregivers of people with dementia. J Affect Disord 2007 Aug;101(1-3):75-89. PMID: 17173977

8. Macdonald A, Cooper B. Long-term care and dementia services: an impending crisis. Age Ageing 2007 Jan;36(1):16-22. PMID:17175565

9. White D. Online support for caregivers. Analysis of an Internet Alzheimer mailgroup. Comput Nurs 2000 Jul-Aug;18(4):168-76. PMID:10939186

10. Boots LM, de Vugt ME, van Knippenberg RJ, Kempen GI, Verhey FR. A systematic review of Internet-based supportive interventions for caregivers of patients with dementia. Int J Geriatr Psychiatry 2014 Apr;29(4):331-44. PMID:23963684 
11. Serafini JD, Damianakis T, Marziali E. Clinical practice standards and ethical issues applied to a virtual group intervention for spousal caregivers of people with Alzheimer's. Soc Work Health Care 2007;44(3):225-43. PMID:17548277

12. Davis Kirsch SE, Lewis FM. Using the World Wide Web in Health-related intervention research. Comput Inform Nurs 2004 Jan-Feb;22(1):8-18. PMID:15069844

13. Hattink B, Meiland $F$, van der Roest $H$, Kevern $P$, Abiuso $F$, Bengtsson J, Giuliano A, Duca A, Sanders J, Basnett F, Nugent C, Kingston P, Dröes RM. Web-based STAR e-learning course increases empathy and understanding in dementia caregivers: results from a randomized controlled trial in the Netherlands and the United Kingdom. J Med Internet Res 2015 Oct;17(10):e241. PMID: 26519106

14. Blom MM, Zarit SH, Groot Zwaaftink RB, Cuijpers P, Pot AM. Effectiveness of an Internet intervention for family caregivers of people with dementia: results of a randomized controlled trial. PLoS One 2015 Feb;10(2):e0116622. PMID:25679228

15. Cristancho-Lacroix V, Moulin F, Wrobel J, Batrancourt B, Plichart M, De Rotrou J, Cantegreil-Kallen I, Rigaud AS. A web-based program for informal caregivers of persons with Alzheimer's disease: an iterative user-centered design. JMIR research protocols 2014 Sep;3(3):e46. PMID:25263541

16. Hayden LJ, Glynn SM, Hahn TJ, Randall F, Randolph E. The use of Internet technology for psychoeducation and support with dementia caregivers. Psychol Serv 2012 May;9(2):215-8. PMID:22662739

17. Kajiyama B, Thompson LW, Eto-Iwase T, Yamashita M, Di Mario J, Marian Tzuang Y, Gallagher-Thompson D. Exploring the effectiveness of an internetbased program for reducing caregiver distress using the iCare Stress Management e-Training Program. Aging Ment Health 2013 Mar;17(5):544-54. PMID:23461355

18. Cristancho-Lacroix V, Wrobel J, Cantegreil-Kallen I, Dub T, Rouquette A, Rigaud AS. A Web-Based Psychoeducational Program for Informal Caregivers of Patients With Alzheimer's Disease: A Pilot Randomized Controlled Trial. J Med Internet Res 2015 May;17(5):e117. PMID:25967983

19. Van der Roest HG, Meiland FJM, Jonker C, Droes RM. User evaluation of the DEMentia-specific Digital Interactive Social Chart (DEM-DISC). A pilot study 
among informal carers on its impact, user friendliness and, usefulness. Aging Ment Health 2010 May;14(4):461-70. PMID:20455122

20. Verhey $F$, de Vugt $M$. Mild cognitive impairment is becoming more psychosocial. Aging Ment Health 2013 Feb;17(3):265-6. PMID:23402425

21. Ducharme F, Dube V, Levesque L, Saulnier D, Giroux F. An Online Stress Management Training Program as a Supportive Nursing Intervention for Family Caregivers of an Elderly Person. Canadian Journal of Nursing Informatics 2011 Jun;6(2):13-44. ISSN 1718-9438

22. Gaugler JE, Kane RL, Kane RA, Newcomer R. Early community-based service utilization and its effects on institutionalization in dementia caregiving. Gerontologist 2005 Apr;45(2):177-85. PMID:15799982

23. Kjallman Alm A, Hellzen O, Norbergh KG. Experiences of long term ongoing structured support in early stage of dementia - a case study. Int J Older People Nurs 2014 Dec;9(4):289-97. PMID:23758956

24. Boots LM, Wolfs CA, Verhey FR, Kempen GI, de Vugt ME. Qualitative study on needs and wishes of early-stage dementia caregivers: the paradox between needing and accepting help. Int Psychogeriatr 2015 Jun;27(6):92736. PMID:25566686

25. Lazarus R, Folkman S. Stress, appraisal and coping. New York: Springer; 1984. ISBN: 9780826141910

26. Bandura A. Self-Efficacy: The Exercise of Control. New York: W.H. Freeman and Company; 1997. ISBN:9780716728504

27. Acton GJ, Kang J. Interventions to reduce the burden of caregiving for an adult with dementia: a meta-analysis. Res Nurs Health 2001 Oct;24(5):349-60. PMID:11746065

28. Toms GR, Quinn C, Anderson DE, Clare L. Help yourself: perspectives on self-management from people with dementia and their caregivers. Qual Health Res 2015 Jan;25(1):87-98. PMID:25192761

29. Lorig KR, Ritter PL, Dost A, Plant K, Laurent DD, McNeil I. The Expert Patients Programme online, a 1-year study of an Internet-based selfmanagement programme for people with long-term conditions. Chronic IIIn 2008 Dec;4(4):247-56. PMID:19091933

30. Forducey PG, Glueckauf RL, Bergquist TF, Maheu MM, Yutsis M. Telehealth for persons with severe functional disabilities and their caregivers: facilitating 
self-care management in the home setting. Psychol Serv 2012 May;9(2):14462. PMID:22662729

31. Craig P, Dieppe P, Macintyre S, Michie S, Nazareth I, Petticrew M. Developing and evaluating complex interventions: the new Medical Research Council guidance. BMJ 2008 Sep;337:a1655. PMID:18824488

32. Sleeswijk Visser F, Stappers PJ, van der Lugt R. Contextmapping: experiences from practice. CoDesign: International Journal of CoCreation in Design and the Arts 2005 Mar;1(2): 119-49. DOI:10.1080/15710880500135987

33. Joosten-Weyn Banningh L. Learning to live with Mild Cognitive Impairment. Development and evaluation of a psychological intervention for patients with Mild Cognitive Impairment, and their significant others. Nijmegen: Radboud University Nijmegen, Medical Centre; 2012. ISBN:9789090266534

34. Denzin N, Lincoln Y. Collecing and Interpreting Qualitative Materials. London: Sage; 1998. ISBN:9781452258041

35. Hennink MM. International focus group research. A handbook for the health and social sciences. Cambridge: University Press; 2007. ISBN:9780521607803

36. Trappenburg J, Jonkman $\mathrm{N}$, Jaarsma $\mathrm{T}$, van Os-Medendorp $\mathrm{H}$, Kort $\mathrm{H}$, de Wit N, Hoes A, Schuurmans M. Self-management: one size does not fit all. Patient Educ Couns 2013 Jul;92(1):134-7. PMID:23499381

37. Mountain GA, Craig CL. What should be in a self-management programme for people with early dementia? Aging Ment Health 2012 Feb;16(5):576-83. PMID: 22360274

38. Lorig KR, Holman H. Self-management education: history, definition, outcomes, and mechanisms. Ann Behav Med. 2003 Aug;26(1):1-7. PMID:12867348

39. Nahm ES, Preece J, Resnick B, Mills ME. Usability of health Web sites for older adults: a preliminary study. Comput Inform Nurs 2004 NovDec;22(6):326-34; quiz 335-6. PMID:15602301

40. Jaspers MW. A comparison of usability methods for testing interactive health technologies: methodological aspects and empirical evidence. Int $\mathrm{J}$ Med Inform 2009 May;78(5):340-53. PMID:19046928 
41. Bowen DJ, Kreuter M, Spring B, Cofta-Woerpel L, Linnan L, Welner D, Bakken S, Kaplan CP, Squiers L, Fabrizio C, Fernandez M. How we design feasibility studies. Am J Prev Med 2009 May;36(5):452-457. PMID: 19362699

42. Winblad B, Palmer K, Kivipelto M, Jelic V, Fratiglioni L, Wahlund LO, Nordberg A, Backman L, Albert M, Almkvist O, Aral H, Basun H, Blennow K, de Leon M, DeCarli C, Erkinjuntti T, Giacobini E, Graff C, Hardy J, Jack C, Jorm A, Ritchie $K$, van Duijn C, Visser P, Petersen RC. Mild cognitive impairment--beyond controversies, towards a consensus: report of the International Working Group on Mild Cognitive Impairment. J Intern Med 2004 Sep;256(3):240-6. PMID:15324367

43. Association AP. Neurocognitive Disorders. 2013. In: Diagnostic and statistical manual of mental disorders (5th ed). Washington DC: American Psychiatric Publishing. doi:10.1176/appi.books.9780890425596

44. Fick DM, Steis MR, Mion LC, Walls JL. Computerized decision support for delirium superimposed on dementia in older adults. J Gerontol Nurs 2011 Apr;37(4):39-47. PMID:21053810

45. Lai CKY, Wong LF, Liu KH, Lui W, Chan MF, Yap LSY. Online and onsite training for family caregivers of people with dementia: results from a pilot study. Int J Geriatr Psychiatry 2013 Jan;28(1):107-8. PMID:23225697

46. Teel CS, Leenerts $\mathrm{MH}$. Developing and testing a self-care intervention for older adults in caregiving roles. Nurs Res 2005 May-Jun;54(3):193-201. PMID:15897795

47. Davis FD. Perceived Usefulness, Perceived Ease of Use, and User Acceptance of Information Technology. MIS Quarterly 1989;13(3):319-40. doi: $10.2307 / 249008$

48. National Alliance for Caregiving and American Association of Retired Persions (NAC \& AARP). Caregiving in the U.S. 2004 Appendix A: Detailed Methodology. Washington $\quad$ D.C.: $2004 . \quad$ URL: http://assets.aarp.org/rgcenter/il/us_caregiving.pdf [accessed 2015-09-15] [WebCite Cache ID 6bZ9dqlex]

49. Campbell SE, Azuara-Blanco A, Campbell MK, Francis JJ, Greene AC, Ramsay CR, Burr JM. Developing the specifications of an open glaucoma screening intervention in the United Kingdom: a Delphi approach. BMC Health Serv Res. 2012 Dec;5:12:447. PMID: 23216983 
50. Lorig K, Chastain RL, Ung E, Shoor S, Holman HR. Development and evaluation of a scale to measure perceived self-efficacy in people with arthritis. Arthritis Rheum. 1989 Jan;32(1):37-44. PMID: 2912463

51. Kiresuk TJ, Sherman RE. Goal attainment scaling: A general method for evaluating comprehensive community mental health programs. Community Ment Health J 1968 Dec;4(6):443-53. PMID:24185570

52. Kiresuk TJ, Smith A, Cardillo JE. Goal attainment scaling: Applications, theory, and measurement. Hillsdale, NJ, England: Lawrence Erlbaum Associates, Inc.; 1994. ISBN:9780898598896

53. Miller WR, Rollnick S. Motivational interviewing: preparing people for change, second edition edn. New York: The Guilford Press; 2002. ISBN:9781572305632

54. Robinson L, Brittain K, Lindsay S, Jackson D, Olivier P. Keeping In Touch Everyday (KITE) project: developing assistive technologies with people with dementia and their carers to promote independence. Int Psychogeriatr 2009 Jun;21(3):494-502. PMID:19193255

55. Savitch N, Zaphris P. Learning from people with dementia to develop research methods for older people. British $\mathrm{HCl}$ Conference: $\mathrm{HCl}$ and the Older Population; Leeds, U.K.2004. URL: http://www.dcs.gla.ac.uk/utopia/workshop/savitch.pdf [accessed 2015-09-15] [WebCite ID 6bZFDrAlz]

56. Hanson E, Magnusson L, Arvidsson H, Claesson A, Keady J, Nolan M. Working together with persons with early stage dementia and their family members to design a user-friendly technology-based support service. Dementia 2007 Aug;6:411-34. doi:10.1177/1471301207081572

57. Barlow J, Wright C, Sheasby J, Turner A, Hainsworth J. Self-management approaches for people with chronic conditions: a review. Patient Educ Couns 2002 Oct-Nov;48(2):177-87. PMID:12401421

58. Van der Vaart R, Witting M, Riper H, Kooistra L, Bohlmeijer ET, van GemertPijnen LJEWC. Blending online therapy into regular face-to-face therapy for depression: content, ratio and preconditions according to patients and therapists using a Delphi study. BMC Psychiatry 2014 Dec;14(355). PMID:25496393

59. Wilhelmsen M, Lillevoll K, Risør MB, Høifødt R, Johansen ML, Waterloo K, Elsemann M, Kolstrup N. Motivation to persist with internet-based cognitive 
behavioural treatment using blended care: a qualitative study. BMC Psychiatry 2013 Nov;13(296). PMID:24199672

60. Ruwaard J, Kok RN. Wild West eHealth: Time to hold our horses? The European Health Psychologist. 2015 Feb;17(1):45-9. ehps.net/ehp

61. Rozental A, Andersson G, Boettcher J, Daniel D, Cuijpers P, Knaevelsrud C. Consensus statement on defining and measuring negative effects of Internet interventions. Internet Interventions $2014 \quad$ Mar;1(1):12-9. doi:10.1016/j.invent.2014.02.001

62. Carlbring P, Andersson, G. Internet and psychological treatment. How well can they be combined? Comput Hum Behav 2006 May;22(3):545-553. doi:10.1016/j.chb.2004.10.009

63. Glueckauf RL, Loomis JS. Alzheimer's Caregiver Support Online: Lessons learned, initial findings and future directions. Neurorehabilitation 2003;18(2):135-46. PMID:12867676

64. Lorig KR, Ritter PL, Dost A, Plant K, Laurent DD, McNeil I. The Expert Patients Programme online, a 1-year study of an Internet-based selfmanagement programme for people with long-term conditions. Chronic IIIn 2008 Dec;4(4):247-56. PMID:19091933

65. Glynn SM, Randolph ET, Garrick T, Lui A. A proof of concept trial of an online psychoeducational program for relatives of both veterans and civilians living with schizophrenia. Psychiatr Rehabil J 2010 Spring;33(4):278-87. PMID:20374986

66. Torp S, Hanson E, Hauge S, Ulstein I, Magnusson L. A pilot study of how information and communication technology may contribute to health promotion among elderly spousal carers in Norway. Health Soc Care Comm 2008 Jan;16(1):75-85. PMID:18181817

67. Stjernsward S, Ostman M, Lowgren J. Online self-help tools for the relatives of persons with depression - a feasibility study. Scand J Caring Sci 2012 Mar;26(1):70-80. PMID:21692824

68. Kim H. Understanding Internet Use Among Dementia Caregivers: Results of Secondary Data Analysis Using the US Caregiver Survey Data. Interact J Med Res 2015 Feb;4(1):e1. PMID:25707033

69. Choi I, Sharpe L, Li S, Hunt C. Acceptability of psychological treatment to Chinese- and Caucasian-Australians: Internet treatment reduces barriers but 
face-to-face care is preferred. Soc Psychiatry Psychiatr Epidemiol 2015 Jan;50(1):77-87. doi:10.1007/s00127-014-0921-1

70. McKechnie V, Barker C, Stott J. The effectiveness of an Internet support forum for carers of people with dementia: a pre-post cohort study. J Med Internet Res 2014 Feb;16(2):e68. PMID:24583789

71. Glueckauf RL, Ketterson TU, Loomis JS, Dages P. Online support and education for dementia caregivers: overview, utilization, and initial program evaluation. Telemed J E Health 2004 Summer;10(2):223-32. PMID:15319052

72. Coulehan MB. Impact of a Web-Based Educational and Peer-Support Intervention for Dementia Caregivers. Aging in America Conference American Society on Aging; 2011 April 27; San Francisco, USA

73. Fledderus M, Schreurs K, Bohlmeijer E, Vollenbroek-Hutten M. Development and Pilot Evaluation of an Online Relapse-Prevention Program Based on Acceptance and Commitment Therapy for Chronic Pain Patients. JMIR Human Factors 2015 Jan;2(1): e1. doi:10.2196/humanfactors.3302

74. Eastman JK, lyer R. The elderly's uses and attitudes towards the Internet. J Consum Marketing 2004;21:208-20. doi:10.1108/07363760410534759

75. Kongsved SM, Basnov M, Holm-Christensen K, Hjollund NH: Response rate and completeness of questionnaires: a randomized study of Internet versus paper-and-pencil versions. J Med Internet Res 2007 Sep 30; 9(3): e25. PMID:17942387 


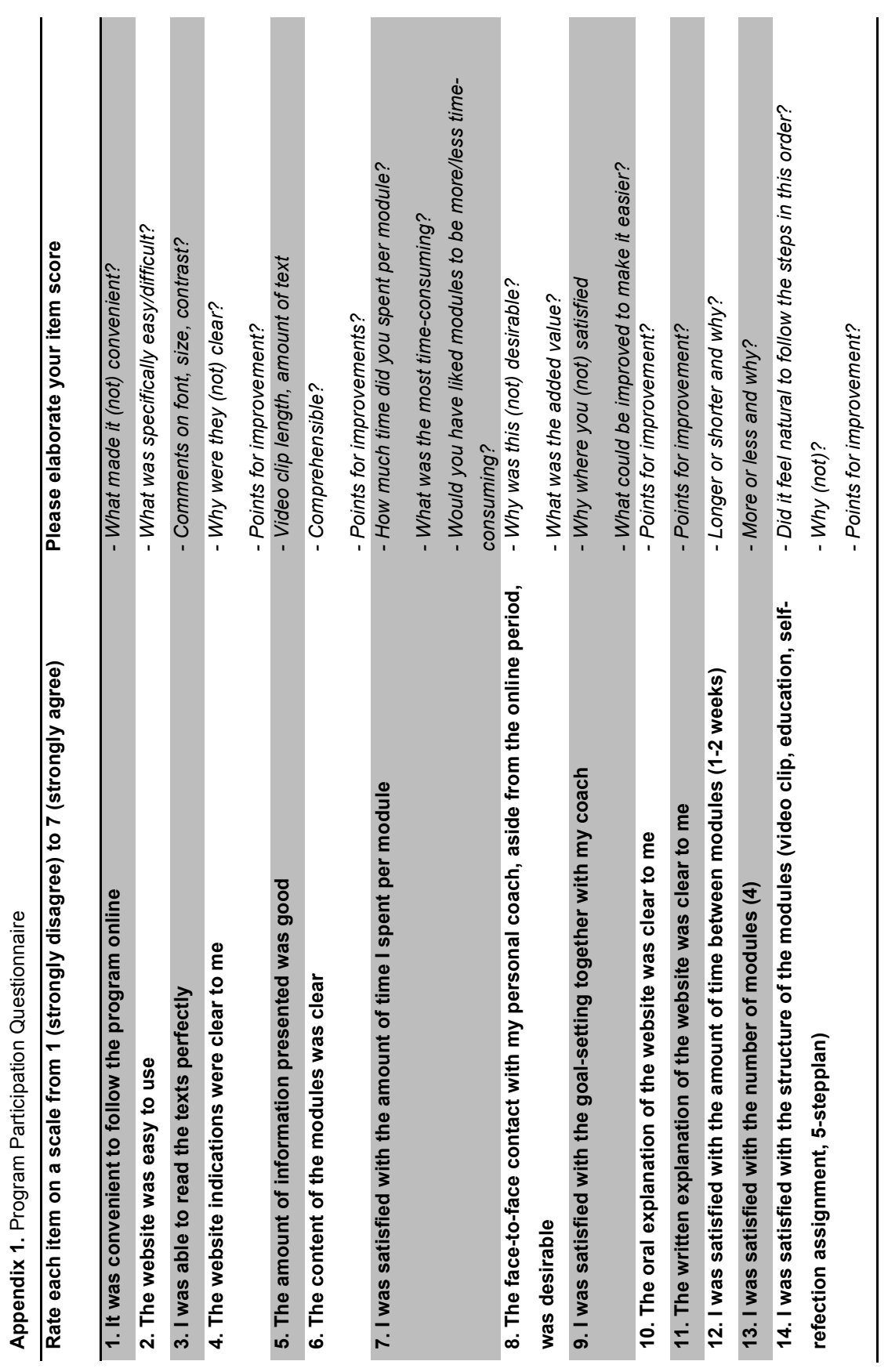




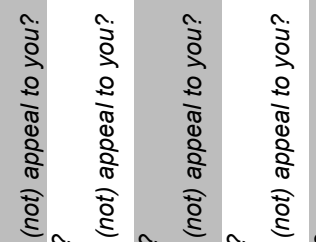

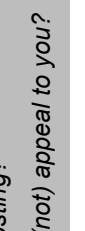

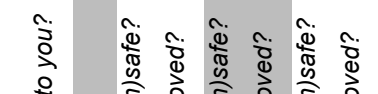

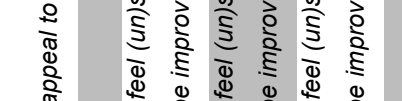

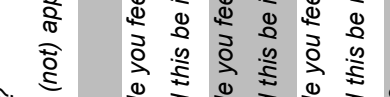

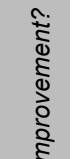

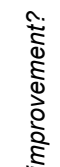

है.

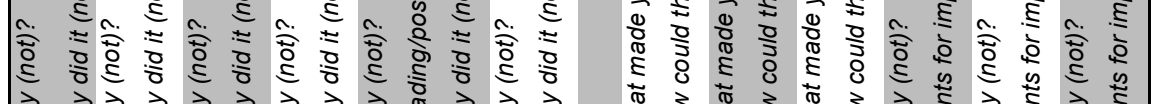

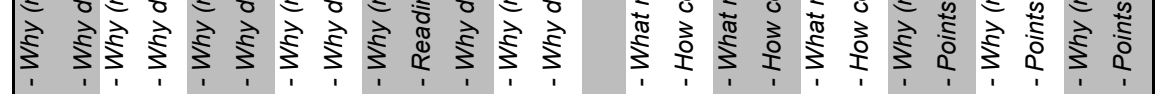

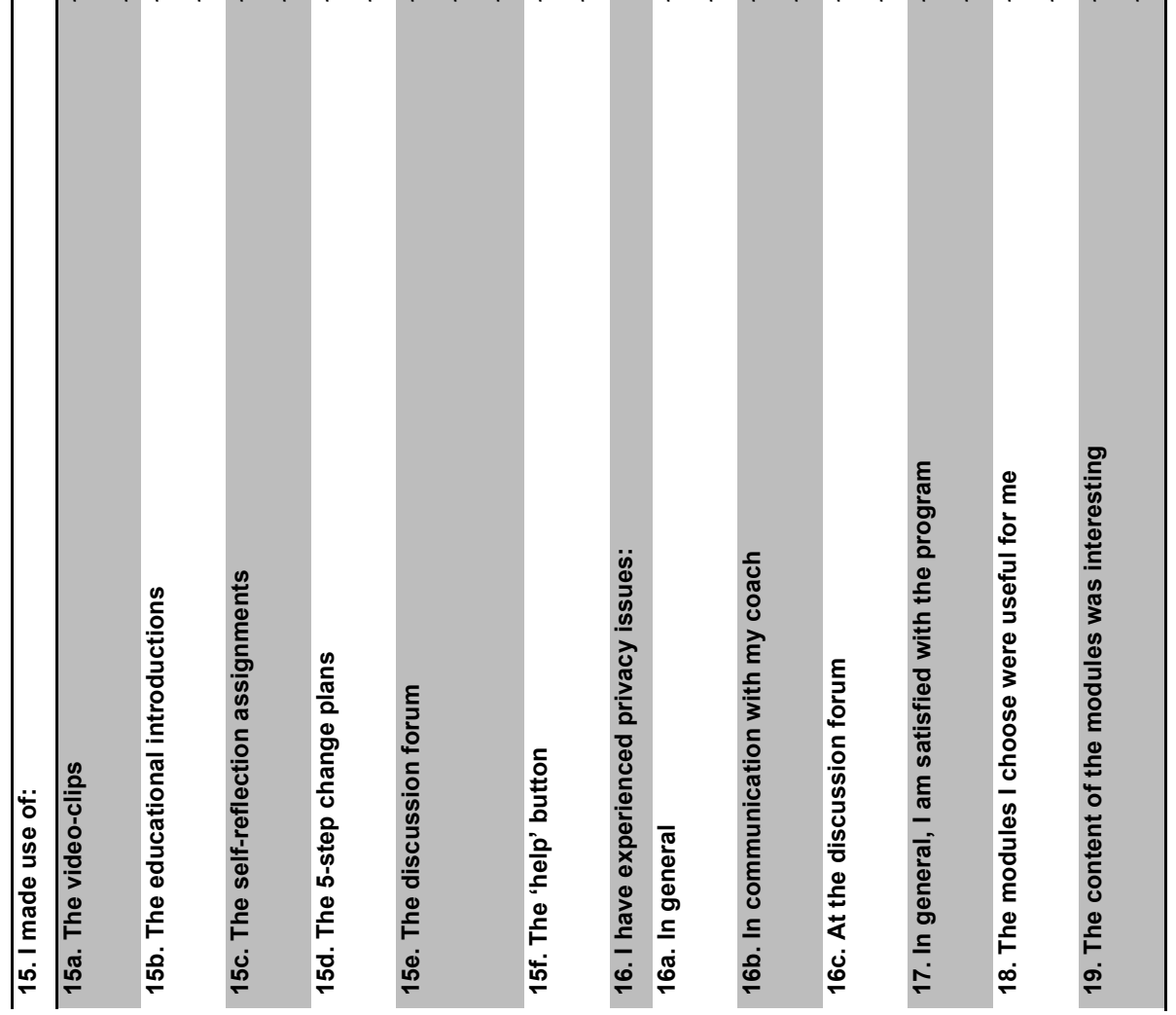




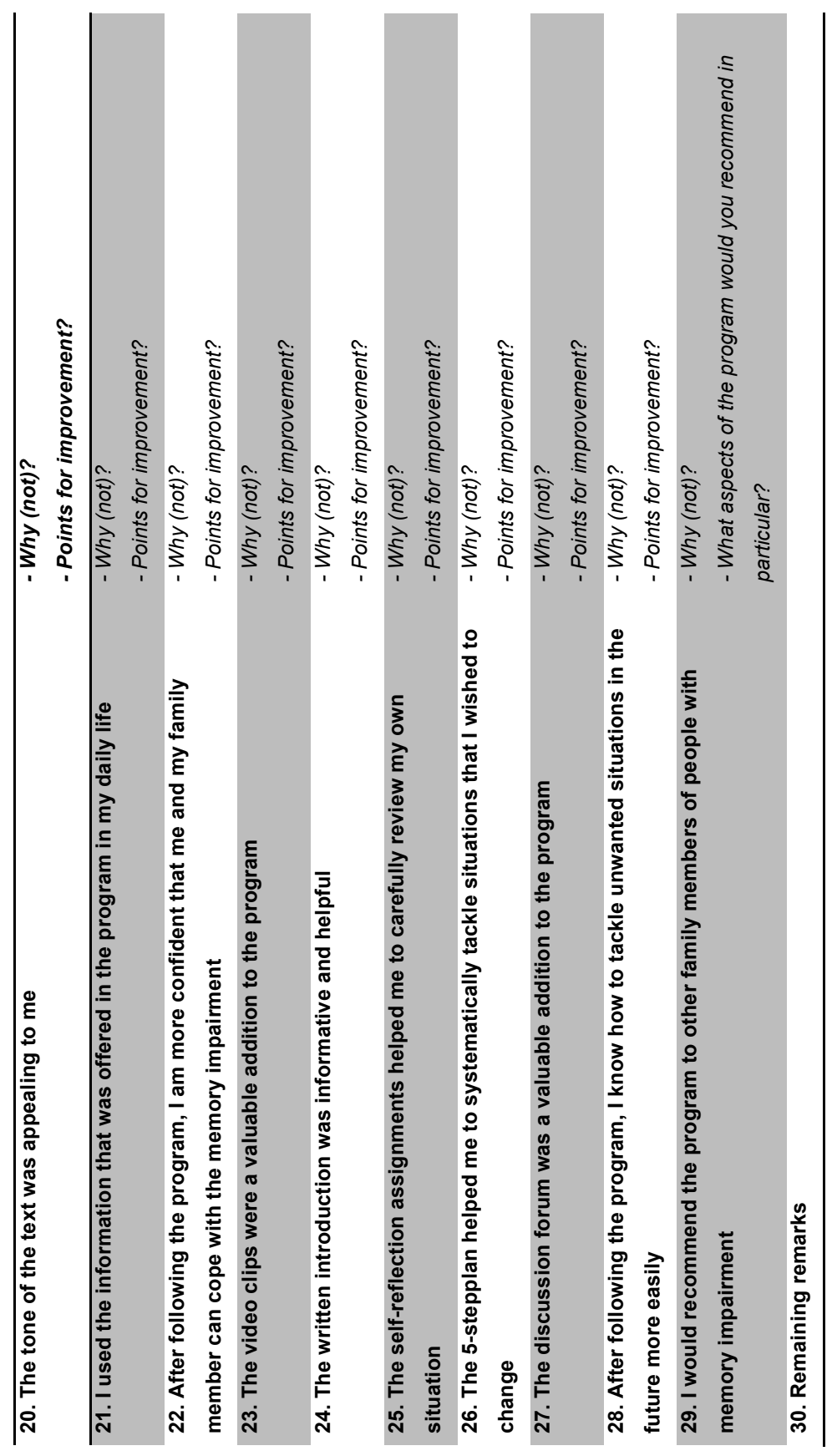






\section{CHAPTER 6}

Effectiveness of the blended care self-management program 'Partner in Balance' for early-stage dementia caregivers: study protocol for a randomized controlled trial

Trials, 2016

Lizzy M.M. Boots, Marjolein E. de Vugt, Gertrudis I.J.M. Kempen \& Frans R.J. Verhey 


\section{ABSTRACT}

Background: The benefits of e-health support for dementia caregivers are increasingly recognized. Reaching early-stage dementia caregivers could prevent high levels of burden and psychological problems in the later stages of dementia. An iterative step-wise approach was employed to develop the blended care selfmanagement program 'Partner in Balance' for early-stage dementia caregivers. The design of a study evaluating the process characteristics and effects is presented.

Methods/design: A mixed method single-blind, randomized controlled trial with 80 family caregivers of community-dwelling people with (very) mild dementia will be conducted. Participants will be randomly assigned to either the 8-week blended care self-management program 'Partner in Balance' or a waiting-list control group. Data will be collected pre- and post-intervention and at 3-, 6- and 12-month follow-ups. Semi-structured interviews will be conducted post-intervention. A process evaluation will investigate the internal and external validity of the intervention. Primary outcomes will include self-efficacy and symptoms of depression. Secondary outcomes will include goal attainment, mastery, psychological complaints (feelings of anxiety and perceived stress), and quality of life. Possible modifying variables such as caregiver characteristics (quality of the relationship, neurotic personality) and intervention aspects (coach) on the intervention effect will also be evaluated. A cost-consequence analysis will describe the costs and health outcomes.

Discussion: We expect to find a significant increase in self-efficacy, goal attainment and quality of life and lower levels of psychological complaints (depression, anxiety and stress) in the intervention group, compared with the control group. If such effects are found, the program could provide accessible care to future generations of earlystage dementia caregivers and increase dementia care efficiency. 


\section{INTRODUCTION}

Family caregivers are currently becoming the main source of care for people with dementia. ${ }^{1}$ However, dementia caregivers are at risk for depression, anxiety and other health problems. ${ }^{2}$ Interventions aimed at reducing the burden of care might reduce dementia care costs in the long term if institutionalization can be postponed. ${ }^{3}$

Many face-to-face caregiver support interventions have proven to be effective on caregiver distress (random effect size $=0.3$ ), caregiver knowledge (random effect size: 0.5), ${ }^{4}$ and self-efficacy (effect sizes ranging from $0.3-0.9$ ), ${ }^{5}$ but the expected future increase in the number of people with dementia raises concerns about whether care professionals can cope with this demand. ${ }^{6}$ E-health interventions could serve as cost-effective alternatives for dementia caregiver support, ${ }^{7-9}$ both increasing caregivers' access to support and extending the reach of such support. ${ }^{10-13}$ The benefits of e-health are increasingly recognized, and remote support for dementia caregivers is growing. ${ }^{14-16}$

Previous e-health studies on dementia caregivers show positive effects on caregiver confidence, stress, depression, and self-efficacy with effect sizes ranging from 0.2-0.3. ${ }^{17}$ Provision of tailored information, a coach and the opportunity to interact with other caregivers shows the most promise. ${ }^{17}$ Blending face-to-face guidance with online modules increases caregivers' connection with the therapist and adherence. ${ }^{18,19}$ However, research on the effects of e-health on dementia caregivers lacks methodological rigor, ${ }^{17}$ and adverse effects of caregiving are often addressed in the later stages of dementia, when caregivers already feel overburdened. ${ }^{20}$ Despite their low levels of life satisfaction and high levels of overload, caregivers do not use services in early stages because they do not feel the need for such services or because they struggle with acceptance due their experience of stigma. ${ }^{20-22}$ Supporting caregivers in an early stage of dementia could prevent high levels of burden and psychological problems in the later stages and delay institutionalization. ${ }^{23-26}$ However, early-stage dementia caregiver support can be experienced adversely if the care does not suit the caregiver's personal situation or the stage of the disease. Negative and stigmatizing information can make it difficult for caregivers to identify with and may hamper acceptance. ${ }^{20}$ Learning to positively manage life with dementia instead of managing the dementia itself in a selfmanagement program could facilitate caregivers' adaptation to their new caregiving role. A focus on enhancing positive, intact experiences that are tailored to the 
individual caregiver's situation may be more effective in increasing caregiver selfefficacy and reducing the negative consequences of caregiving at later stages. ${ }^{20}$

\section{Study aim and hypotheses}

The current paper presents the design of a randomized waiting-list controlled trial investigating the effects of the blended care self-management program 'Partner in Balance'. Alongside the effectiveness study, a process evaluation will be conducted to evaluate internal and external validity. As recommended by the Medical Research Council (MRC) Framework for the design and evaluation of complex intervention, ${ }^{27}$ the program was based on existing literature, ${ }^{17}$ theory, and user and professional input. $^{28}$ In a pilot evaluation, caregivers reported increased feelings of self-efficacy and goal attainment post-intervention. Feedback from the feasibility study was used to adapt the intervention to increase user-friendliness. ${ }^{28}$ The next objective is to evaluate the process characteristics, effectiveness and cost-consequence of 'Partner in Balance'. Specifically, we aim to investigate (1) the internal and external validity of the intervention based on sampling quality (recruitment, randomization and reach) and intervention quality (relevance, feasibility, and performance according to protocol) prior to the effect analysis to evaluate credibility and generalizability, ${ }^{29}$ (2) whether 'Partner in Balance' is superior to the waiting-list control condition in terms of participants' subjective well-being, as evidenced by improved subjective selfconfidence (increased self-efficacy) and goal attainment and lower levels of psychological complaints (depression, anxiety and stress) following the selfmanagement intervention, and (3) whether the effects are maintained after 3, 6 and 12 months. Lastly, (4) the cost-consequence of Partner in Balance is calculated to estimate the impact of the intervention on resource use, costs, and health outcomes.

\section{METHODS}

The Medical Ethics Committee of the Maastricht University Medical Center+ approved this study (\#12-4-059). The study is a randomized waiting-list controlled trial. This study was designed to maximize acceptability and adherence to the research protocol in the control group and minimize attrition effects. Data will be collected pre- and post-intervention and at 3-, 6- and 12-month follow-ups. See Figure 1 for the flow diagram. 


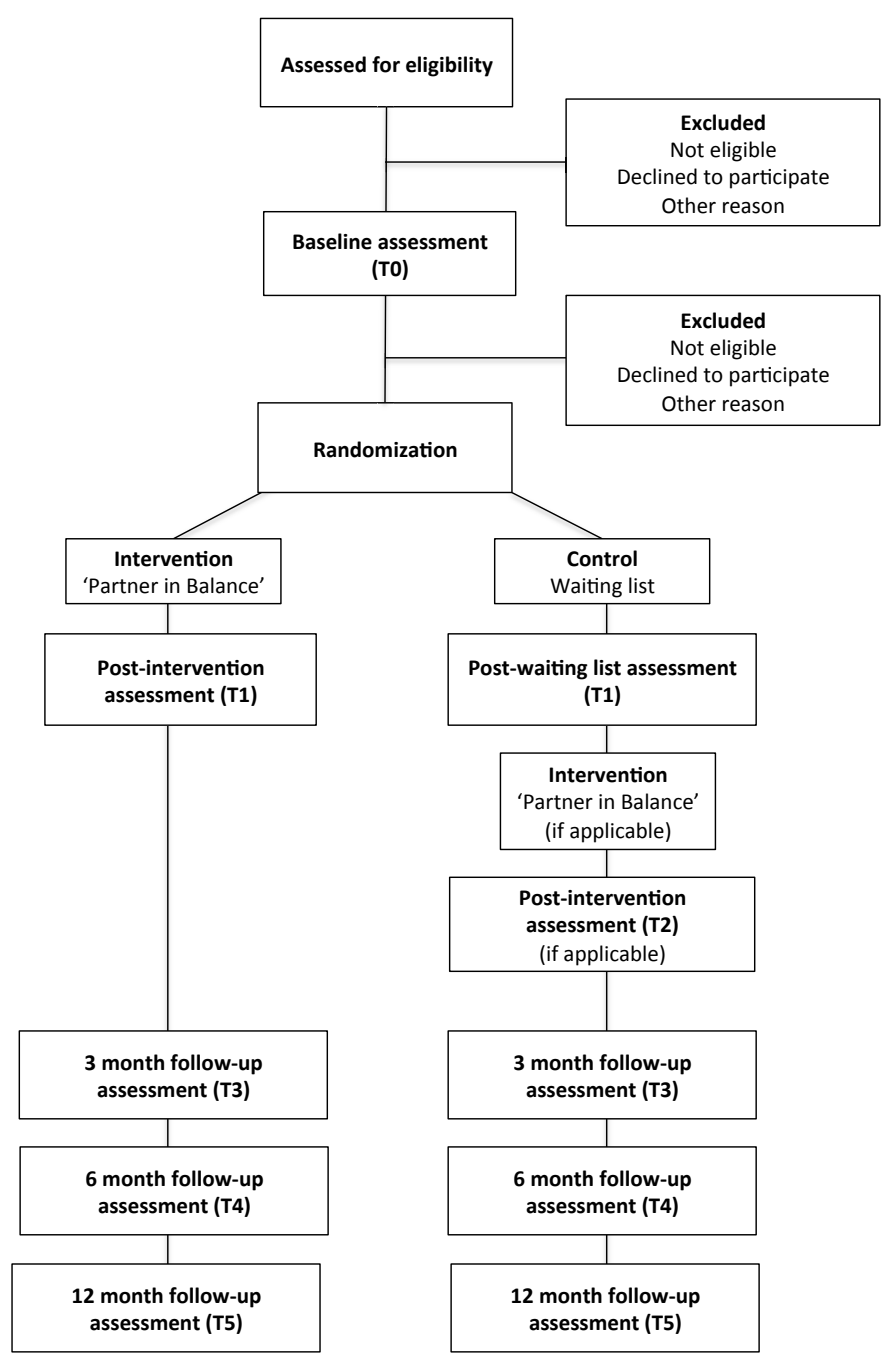

Figure 1. CONSORT flow diagram

\section{Population}

\section{Recruitment}

The study population will consist of family caregivers of community-dwelling people with (very) mild dementia of all subtypes (Clinical Dementia Rating (CDR) score 0.51). ${ }^{30}$ No age limit will be applied; however, only adult family caregivers will be solicited. Participants will be recruited from memory clinics (MUMC+, Elkerliek Hospital Helmond, Catharina Hospital Eindhoven) and ambulatory mental health clinics (Virenze-RIAGG Maastricht, MET ggz Roermond) in the south of the 
Netherlands. Caregivers will be approached by the clinician or therapist who is treating their family member. Prior to participating, caregivers will provide written informed consent.

\section{Eligibility}

Family caregivers of people with (very) mild dementia of all subtypes (CDR score $0.5-1$ ) who are $>18$ years, have access to the Internet at home, and have basic skills in the use of computers are eligible to participate in the study. Potential participants that have insufficient cognitive abilities to engage in the online self-management program; are overburdened or have severe health problems, as determined by study staff; or care for people with dementia caused by human immunodeficiency virus (HIV), acquired brain impairment, Down syndrome, chorea associated with Huntington's disease or alcohol abuse will be excluded from participation.

\section{Randomization}

Following the baseline assessment, participants will be randomly assigned to either the self-management intervention or the waiting-list control group using a computer program operated by an independent statistician. Block randomization will be conducted to reduce the risk of unbalanced assignment to the experimental and control groups. We will use randomly permuted blocks and several block sizes $(4,6$ and 8). The block size and order of allocation will be randomly chosen at the beginning of each block. This reduces the risk of predicting group assignment and keeps research staff blind to the randomization process. An independent research assistant who is unknown to the allocation of the treatment will conduct the postintervention and follow-up assessments, and will be asked to evaluate success of blinding and reason for possible unmasking on the Case Record Form.

\section{Intervention}

\section{Experimental group}

The 'Partner in Balance' intervention consists of a face-to-face intake session with a personal coach, an online period, and a face-to-face evaluation session with the personal coach. The basis of the intervention is learning to identify areas of change and creating personal goals. The development and final intervention are described in detail elsewhere. ${ }^{26}$ The goal of the intake session is to familiarize participants with the program and set goals that they wish to accomplish through their participation 
using the motivational interviewing technique frequently used to identify change objectives and enhance intrinsic motivation to change. ${ }^{31}$ Goals and strategies to achieve these goals are individually determined and depend on the participants' personal problems, motivation, and capabilities. Given that it is not usual for elderly to reflect on their problems or concerns thematically, a 'toolbox' of themes should be developed to aid the discussion of these issues with the participants. ${ }^{32}$ Based on their personal needs and areas of interest, participants will select four out of nine modules in the toolbox, and they will be briefed individually to ensure that they understand the online procedure. The module themes are provided in Box 1 . The module contents are described elsewhere. ${ }^{28}$ Following the intake, participants will complete their chosen modules online during an 8-week period. Two weeks are allocated for each module, but participants will be allowed to complete the modules at their own pace in accordance with the self-management approach. ${ }^{33}$ After the online period, participants will meet with their coach face-to-face to discuss their ability to accomplish goals and cope with future difficulties. Participants will continue to have access to their personal page and modules after the intervention period.

Box 1. Themes of the available 'Partner in Balance' modules

\begin{tabular}{|c|c|}
\hline & Partner in Balance modules \\
\hline 1. & Acceptance \\
\hline 2. & Balance in activities \\
\hline 3. & $\begin{array}{l}\text { Communication with family member } \\
\text { and environment }\end{array}$ \\
\hline 4. & Coping with stress \\
\hline 5. & Focusing on the positive \\
\hline & Insecurities and rumination \\
\hline & Self-understanding \\
\hline & The changing family member \\
\hline & Social relations and support \\
\hline
\end{tabular}

The personal coach

The personal coach is an experienced professional (psychologist or psychiatric nurse) from one of the participating sites. Coaches will receive a one-day training on self-management techniques and online help before the start of the intervention. They will receive supervision from an experienced professional in the fields of psychology and self-management to ensure high-quality feedback. 
Coaches support participants in choosing modules that fit their situation, help identify feasible goals, offer techniques to achieve goals and provide general constructive feedback on their assignments. Coaches will be matched with the participants assigned to them via a personal login code.

\section{Control group}

Participants in the control group will be placed on a waiting list for 8 weeks. After they complete the post-test assessment, they will receive the online self-management intervention. They will receive the same pre-test and post-test attention from the research team as the experimental group.

\section{Procedure}

Participants will be assessed at 5 time points: $\left(T_{0}\right)$ baseline assessment, $\left(T_{1}\right)$ postintervention or waiting list assessment (8 weeks), $\left(T_{2}\right)$ post-intervention assessment for waiting-list (8 weeks after $\left.T_{1}\right),\left(T_{3}\right) 3$-month follow-up, $\left(T_{4}\right)$ 6-month follow-up, and ( $T_{5}$ ) 12-month follow-up. Figure 2 shows the schedule of enrollment, procedures and mixed method assessments per condition.

\section{Questionnaires}

\section{Primary outcomes}

The Caregiver Self-efficacy Scale (CSES) ${ }^{34}$ is based on a Dutch version of Lorig et al.'s ${ }^{35}$ self-efficacy instrument and measures care management self-efficacy (4 items) and service use self-efficacy (5 items). Item scores range from 1 (not at all certain) to 10 (very certain). Total care management self-efficacy scores range from 4-40 and service use self-efficacy from 5-50. Good reliability was demonstrated for the CSES in a previous study. ${ }^{28}$

The Dutch version of the Center for Epidemiological Studies Depression Scale (CES$D)^{36}$ consists of 20 items that rate the frequency of depressive symptoms during the past week, with higher total scores indicating greater depressive symptoms. Scores range from 0 (rarely or none of the time [less than 1 day]) to 3 (most or all of the time [5 to 7 days]). The total score ranges from 0 to 60 . Items represent depressed mood, feelings of guilt and worthlessness, feelings of helplessness and hopelessness, psychomotor retardation, loss of appetite, and sleep disturbance. The CES-D is sensitive to changes in caregiver depressive symptoms after intervention. ${ }^{37}$ 


\begin{tabular}{|c|c|c|c|c|c|c|c|}
\hline & \multicolumn{7}{|c|}{ STUDY PERIOD } \\
\hline & \multirow{2}{*}{$\begin{array}{c}\text { Enrolment } \\
-T_{1}\end{array}$} & \multirow{2}{*}{$\begin{array}{c}\text { Baseline } \\
T_{0}\end{array}$} & \multicolumn{5}{|c|}{ Post-allocation } \\
\hline TIMEPOINT $^{* *}$ & & & $T_{1}$ & $T_{2}$ & $T_{3}$ & $T_{4}$ & $T_{5}$ \\
\hline \multicolumn{8}{|l|}{ ENROLMENT: } \\
\hline Eligibility screen & $x$ & & & & & & \\
\hline Informed consent & $\mathrm{X}$ & & & & & & \\
\hline Allocation & & $X$ & & & & & \\
\hline Demographics & & $x$ & & & & & \\
\hline \multicolumn{8}{|l|}{ INTERVENTION: } \\
\hline Partner in Balance & & $\mathrm{X}$ & $x$ & & $x$ & $x$ & $\mathrm{X}$ \\
\hline \multirow{2}{*}{$\begin{array}{r}\text { Waiting-list } \\
\text { Waiting-list + Partner } \\
\text { in Balance }\end{array}$} & & $\mathrm{X}$ & $x$ & & $x$ & $\mathrm{x}$ & $\mathrm{X}$ \\
\hline & & & & & & & \\
\hline \multicolumn{8}{|l|}{ ASSESSMENTS: } \\
\hline CSES & & $\mathrm{X}$ & $x$ & $x$ & $x$ & $\mathrm{x}$ & $\mathrm{X}$ \\
\hline CES-D & & $x$ & $x$ & $x$ & $x$ & $x$ & $X$ \\
\hline GAS & & $x$ & $x$ & $x$ & & & \\
\hline PMS & & $\mathrm{X}$ & $x$ & $x$ & $x$ & $x$ & $X$ \\
\hline PSS & & $x$ & $x$ & $x$ & $x$ & $x$ & $x$ \\
\hline$H A D S-A$ & & $x$ & $x$ & $x$ & $x$ & $x$ & $X$ \\
\hline ICECAP-O & & $x$ & $x$ & $x$ & $x$ & $x$ & $x$ \\
\hline Relationship quality & & $x$ & $x$ & $x$ & $x$ & $x$ & $X$ \\
\hline NEO-FFI & & $x$ & $x$ & $x$ & $x$ & $x$ & $x$ \\
\hline $\begin{array}{r}\text { Semi-structured } \\
\text { interview }\end{array}$ & & & $x$ & $x$ & & & \\
\hline RUD-lite & & $\mathrm{X}$ & $x$ & $x$ & $x$ & $\mathrm{x}$ & $x$ \\
\hline
\end{tabular}

Figure 2. Schedule of enrolment and assessments per condition

\section{Secondary outcomes}

Goal attainment scaling (GAS) ${ }^{38}$ is a measure of treatment-induced change. GAS enables comparisons of individuals' relative success in achieving goals that are individually determined. Scores range from -2 (much less than expected) to +2 (much better than expected), with 0 indicating that the goal is attained. Raw scores are transformed into T-scores. ${ }^{39}$ T-scores include attainment level and a potential weight assigned to the goal(s). T-scores of $\geq 50$ indicate effective goal attainment. GAS demonstrated reliable monitoring of progress over time. ${ }^{40}$ 
The Pearlin Mastery Scale (PMS) ${ }^{41}$ measures perceived control, otherwise known as mastery. The scale includes 7 items, with scores ranging from 1 (completely agree) to 5 (completely disagree). The sum score (range 0-35) indicates the extent of perceived control or mastery, with higher scores indicating greater perceived control. The psychometric properties of the Dutch version of the Mastery Scale have found to be good in previous studies. ${ }^{42}$

The Perceived Stress Scale (PSS) ${ }^{43}$ measures overall appraisals of stress in the past month. Ten items on unpredictability, control and overload are rated on a 5-point scale from 0 (never) to 4 (very often), with higher total scores indicating higher levels of stress. The scale showed good reliability. ${ }^{44}$

The Hospital and Anxiety Depression Scale (HADS-A) ${ }^{45}$ is used to generate scores for generalized anxiety. The anxiety subscale consists of 7 items rated from 0 (not at all) to 3 (a great deal of the time). Total scores range from 0 to 21 , with higher scores indicating more anxiety. Good reliability was found. ${ }^{46}$

The Investigating Choice Experiments for the Preferences of Older People CAPability measure for Older people (ICECAP-O $)^{47}$ measures five important attributes of quality of life. The value system for the 1024 states defined by the instrument was derived from a survey of older people in England, using a best-worst scaling valuation method. The value system provides a single summary score, anchored at zero ('no capability') and 1.0 ('full capability'), for each state described in terms of the five attributes. The ICECAP-O may have the potential to measure broader outcomes and be more sensitive to differences between intervention and comparators than the EQ$5 \mathrm{D}$, which is often used in cost-effect evaluations. ${ }^{48}$

\section{Additional measures}

Quality of the relationship is rated with four items of the University of Southern California Longitudinal Study of Three-Generation Families measures of positive affect. $^{49}$ The items indicate a) general closeness, b) communication, c) similarity of views about life and d) degree of getting along. Scores range from 1 (not at all) to 4 (very). The value system for the 1024 states uses a best-worst scaling valuation method, providing a single summary score, anchored at zero ('no capability') and 1.0 ('full capability'). Good reliability was found in a previous study. ${ }^{50}$ 
The NEO Five-Factor Inventory (NEO-FFI) ${ }^{51}$ measures personality. The 12-item Neuroticism domain will be used to identify individuals who are prone to psychological distress. This domain assesses 6 traits: anxiety, angry hostility, depression, self-consciousness, impulsiveness and vulnerability. ${ }^{52}$ The reliability of the Dutch version of the NEO-FFI is good. ${ }^{51}$

\section{Semi-structured interview}

After the intervention is completed, a semi-structured interview will be conducted to qualitatively evaluate the effect of the program on participants' self-efficacy. The interview will take place face-to-face in the caregiver's home or at the participating institution and will be audiotaped with the participants' permission. Topics include the application of the intervention in daily life and the intervention's impact on knowledge about the disease, caregiver functioning, and self-esteem.

\section{Process outcomes}

Prior to the analysis of effects, the internal and external validity of the intervention will be evaluated using data on sampling quality (recruitment, randomization, and reach) and intervention quality (relevance, feasibility, and performance according to protocol) to provide essential information on the program's credibility and generalizability. ${ }^{29} \mathrm{~A}$ description of the recruitment and randomization procedure, the informed consent and allocation procedure, and the barriers and facilitators to recruiting caregivers will be provided. Reach will be determined by the proportion of caregivers participating in the study and the number of institutions involved in the intervention. Intervention quality will be determined on two levels, (1) coach and (2) participant. Data will be collected from the research database, and during focus group interviews with coaches and individual semi-structured interviews with participants post-intervention. Coaches will be asked to complete a questionnaire about clearness, relevance, and usability of the intervention. Delivery of the intervention according to protocol will be evaluated with a structured registration form (Appendix I). For an overview of the specific methods used for each aspect of validity, see Table 1. 
Table 1. Methods used to assess process of the intervention

\begin{tabular}{ll}
\hline Process Aspects & Methods used \\
\hline Sampling quality & Procedure of recruitment, informed \\
& consent, and allocation; recruitment \\
& barriers and facilitators; reach \\
(proportion participants/institutions)
\end{tabular}

Intervention quality (rated by coach)

Protocol deviations; amount/intensity contact with participant

Relevance for caregivers + professional coaches; usability in daily practice

Advantages of the program; disadvantages/suggestions for improvement; recommendation to other professionals

Intervention quality (rated by participant)

Number of online visits and time spent per module

Compliance to the program

Clarity of the content; website ease of use; satisfaction with online aspect combined with face-to-face contact; satisfaction with personal coach and feedback; advantages of the program; disadvantages/suggestions for improvement; impact on understanding and knowledge; impact on caregiver self-esteem; recommendation to other caregivers

a 1 = totally disagree to 5 = totally agree

${ }^{b}$ Online system usage data include frequency of logging in to the system, length of visit (per module feature), and time and date of visit.

\section{Intervention costs}

To provide a comprehensive presentation of the value of Partner in Balance, a costconsequence analysis will be conducted. This is a listing of all the relevant costs and consequences (outcomes) of the intervention. Costs will be based on the Dutch guidelines for cost calculations in health care. ${ }^{53}$ Formal and informal resources used by the caregiver and care recipient will be mapped by means of The Resource Utilization in Dementia - shortened version (RUD-lite), ${ }^{54}$ which includes information on hospital costs, contacts with the GP or other health care professionals, home care, day care, admissions to a nursing home or elderly home, medication and acquisition of goods/aids. Costs are calculated by multiplying the volume of resource use by the cost price per resource unit and include the period from the baseline assessment up to the last follow-up measurement (12 months). Intervention costs 
include time spent on the intake and evaluation session, e-mail contact, the coach training sessions, materials, and coach supervision. The coaches will register the amount of time spent on the intake, evaluation session and e-mail contact on a structured registration form. All costs are expressed in Euros and are adopted from Hakkaart. $^{53}$

\section{Sample size}

We determined our sample size based on previous online intervention studies on caregivers of people with dementia using the Caregiver Self-efficacy Scale (CSES) as outcome measure, the use of repeated measures, within-between interaction with a mean effect size of $0.2,{ }^{55}$ the following assumptions: alpha 0.05 , power $85 \%$ and $25 \%$ loss to follow-up. In accordance with these criteria, we aim to enroll 80 participants (40 participants per group).

\section{Statistical analyses}

\section{Quantitative measures}

Prior to the analysis, data will be checked for missing values, outliers, and normality. Possible differences between the study groups' baseline characteristics will be tested with t-tests for continuous variables and $X^{2}$-tests for categorical variables. In case of missing data, we intend to test if data is missing completely at random (MCAR) based on a comparison of the baseline characteristics of study completers and participants with missing values. If $p<.05$ for (one of) the variables in the model, the missing values are non-random. In case of data MCAR, the specification of a missing value or dropout model is not necessary and list wise deletion will be applied. In case of missing data not at random, we will apply a multiple imputation-based strategy. ${ }^{56}$

To examine differences in the outcomes of the intervention and the waiting-list control groups during the intervention period, an analysis of covariance (ANCOVA) will be conducted with outcome at post-intervention as the dependent variable, intervention (Partner in Balance, waiting-list control group) as the between-subjects variable and the pre-intervention outcome, emotional instability, quality of the relationship, educational level and relationship to the care recipient as covariates. Each outcome will be assessed as a dependent variable. Group differences in the post-intervention outcome adjusted for its baseline value will be examined to test the primary hypothesis. If differences are found, the inter-group effect size will be calculated according to Cohen's $d$. 
To analyze changes in the primary and secondary outcomes during the total study period, the data from the intervention-only group and the waiting-list group receiving the intervention after 8 weeks will be combined using a linear mixed model (LMM). The LMM will estimate fixed effects (regression slopes) for the change during the waiting-list interval (T0-T1 as measured in the waiting-list group only) and fixed effects for change in the intervals during (T1-T2) and after (T2-T3, T3-T4, T4-T5) the intervention period (measured in the waiting list group after the first 8 weeks and in the intervention group). This will allow us to compare the rate of change in those receiving no intervention (T0-T1) with those receiving the intervention (T1-T5) while accounting for the fact that data are nested in individuals and, therefore, correlated. Intervals will be entered as a categorical variable (5 levels) using dummies. Model fit of models with random intercepts (at the participant level) and models with random intercepts and random slopes (at the interval level) will be compared using likelihood ratio tests.

Emotional instability and quality of the relationship will be included as covariates because they are expected to influence the difference between groups. Coach will be introduced into the analysis as a random factor to estimate the variability attributable to the coach. For goal attainment, descriptive statistics will be used to calculate the total number of goals set per domain. Mean goal attainment scaling scores (T-scores) will be calculated with a standard formula ${ }^{38}$ for each measurement time point.

All analyses will be carried out according to the intention to treat principle using IBM SPSS statistics 22.0 for Macintosh. All tests of significance will report mean change and will be two tailed, with $\alpha$ set at 0.05 .

\section{Semi-structured questionnaire and process evaluation}

The qualitatively obtained data from the semi-structured interview will be transcribed verbatim. Two independent researchers will perform deductive content analysis of the transcribed text using ATLAS.ti. Conceptual labels will be assigned to textual fragments and organized into categories. Categories will be merged into common themes in a consensus meeting. Disagreements will be solved through discussion. The quantitative items scored by coaches will be calculated by means of descriptive statistics. 


\section{Monitoring and participant safety}

Monitoring of the recruitment and execution of the study will be conducted by the trial monitoring committee of the MUMC+ (Clinical Trial Center Maastricht). Adverse events (AEs) and serious adverse events (SAEs) are not anticipated but cannot be ignored. If participants drop out, they will be asked if they had experienced an adverse or harmful event during the study period that could be attributed to 'Partner in Balance'. Included participants will be asked the same question during the postintervention assessment and at the 3-, 6- and 12-month follow-ups. All AEs and SAEs will be recorded. SAEs will be reported to the accredited MEC that approved the protocol. AEs will be followed until they have abated or until a stable situation has been reached. Depending on the event, follow-up may involve additional tests or medical procedures, as indicated, and/or referral to the general physician or a medical specialist. If participants do not agree to this procedure, they cannot participate in the study.

\section{DISCUSSION}

In this paper, we described the design of a randomized waiting-list controlled trial to evaluate the effectiveness and process of a blended self-management program to improve caregiver self-efficacy and psychological well-being. As recommended by the MRC Framework, ${ }^{27}$ this study was preceded by an iterative development and feasibility study. ${ }^{28}$ There are several unique aspects of the current study. To our knowledge, this is the first blended care intervention for early-stage dementia caregivers developed with potential users and tailored to the individual user. Furthermore, the MRC Framework suggests that implementation should be considered during the first phases of intervention development and evaluation. ${ }^{27}$ Implementation is expected to be successful because this intervention was developed with potential professional users and will be evaluated in multiple institutions and with coaches from different backgrounds. The program will be delivered in daily practice and can be integrated in the present care provided. Furthermore, tailoring to individual caregiver needs is expected to increase program effectiveness $^{17}$ and facilitate implementation. ${ }^{57}$ Additionally, GAS enables identification of individual benefits that caregivers experience beyond the generalized measures. ${ }^{58}$ The process evaluation facilitates our understanding of the quantity and quality of intervention delivered and evaluates the generalizability of the research based on an understanding of the context. ${ }^{59}$ The mixed methods approach enables 
better understanding of whether and how the intervention works, and facilitates replication of the intervention through greater knowledge of the active component(s) and potential barriers to implementation. ${ }^{60}$ Because all the resources used, costs and outcomes are transparently listed in the cost-consequence analysis, decision makers can select the information that is of most interest to them. Finally, the waiting-list controlled design of the study allows all potentially interested participants to participate in the intervention program. It may increase caregivers' motivation to participate given that usual care for (very) mild dementia caregivers often either does not include counseling or includes only very infrequent counseling. ${ }^{61}$

In conclusion, the results of this study will be a valuable contribution to the growing knowledge on e-health for dementia caregivers. Partner in Balance is expected to be effective in increasing caregiver self-efficacy and reducing depressive symptoms in early-stage dementia caregivers. The study will also provide insight into program delivery and program costs related to the program consequences. The results will be used to inform clinicians and researchers of the delivery, costs and effects of Partner in Balance as a tool to support dementia caregivers. 


\section{References}

1. Alzheimer's Association: Alzheimer's disease facts and figures. Alzheimers Dement 2014, 10(2).

2. Brodaty $\mathrm{H}$, Donkin M: Family caregivers of people with dementia. Dialogues Clin Neurosci 2009, 11(2):217-228.

3. Olazaran J, Reisberg B, Clare L et al: Nonpharmacological therapies in alzheimer's disease: A systematic review of efficacy. Dement Geriatr Cogn Disord 2010, 30(2):161-178.

4. Brodaty $\mathrm{H}$, Green A, Koschera A: Meta-analysis of psychosocial interventions for caregivers of people with dementia. J Am Geriatr Soc 2003, 51(5):657-664.

5. Tang WK, Chan CY: Effects of psychosocial interventions on self-efficacy of dementia caregivers: A literature review. Int J Geriatr Psychiatry 2015.

6. Macdonald A, Cooper B: Long-term care and dementia services: An impending crisis. Age Ageing 2007, 36(1):16-22.

7. Glueckauf RL, Loomis JS: Alzheimer's caregiver support online: Lessons learned, initial findings and future directions. Neurorehabilitation 2003, 18(2):135-146.

8. Martin-Carrasco M, Martin MF, Valero CP, Millan PR, Garcia Cl, Montalban SR, Vazquez AL, Piris SP, Vilanova MB: Effectiveness of a psychoeducational intervention program in the reduction of caregiver burden in alzheimer's disease patients' caregivers. Int J Geriatr Psychiatry 2009, 24(5):489-499.

9. Lewis BA, Williams DM, Neighbors CJ, Jakicic JM, Marcus BH: Cost analysis of internet vs. Print interventions for physical activity promotion. Psychol Sport Exerc 2010, 11(3):246-249.

10. Davis Kirsch SE, Lewis FM: Using the world wide web in health-related intervention research. CIN: Computers, Informatics, Nursing 2004, 22(1):8-18.

11. Harrell KM, Wilkins SS, Connor MK, Chodosh J: Telemedicine and the evaluation of cognitive impairment: The additive value of neuropsychological assessment. J Am Med Dir Assoc 2014, 15(8):600-606.

12. Serafini JD, Damianakis T, Marziali E: Clinical practice standards and ethical issues applied to a virtual group intervention for spousal caregivers of people with alzheimer's. Soc Work Health Care 2007, 44(3):225-243.

13. Robinson L, Brittain K, Lindsay S, Jackson D, Olivier P: Keeping in touch everyday (kite) project: Developing assistive technologies with people with 
dementia and their carers to promote independence. Int Psychogeriatr 2009, 21(3):494-502.

14. Cheng ST, Lau RW, Mak EP, Ng NS, Lam LC, Fung HH, Lai JC, Kwok T, Lee DT: A benefit-finding intervention for family caregivers of persons with alzheimer disease: Study protocol of a randomized controlled trial. Trials 2012, 13:98.

15. Blom MM, Zarit SH, Groot Zwaaftink RB, Cuijpers P, Pot AM: Effectiveness of an internet intervention for family caregivers of people with dementia: Results of a randomized controlled trial. PLoS One 2015, 10(2):e0116622.

16. Cristancho-Lacroix V, Wrobel J, Cantegreil-Kallen I, Dub T, Rouquette A, Rigaud AS: A web-based psychoeducational program for informal caregivers of patients with alzheimer's disease: A pilot randomized controlled trial. J Med Internet Res 2015, 17(5):e117.

17. Boots LM, de Vugt ME, van Knippenberg RJ, Kempen GI, Verhey FR: A systematic review of internet-based supportive interventions for caregivers of patients with dementia. Int J Geriatr Psychiatry 2014, 29(4):331-344.

18. van der Vaart R, Witting M, Riper H, Kooistra L, Bohlmeijer ET, van GemertPijnen LJEWC: Blending online therapy into regular face-to-face therapy for depression: Content, ratio and preconditions according to patients and therapists using a delphi study. BMC Psychiatry 2014, 14(355).

19. Wilhelmsen M, Lillevoll K, Risør MB, Høifødt R, Johansen ML, Waterloo K, Eisemann $\mathrm{M}$, Kolstrup $\mathrm{N}$ : Motivation to persist with internet-based cognitive behavioural treatment using blended care: A qualitative study. BMC Psychiatry 2013, 13(296).

20. Boots LM, Wolfs CA, Verhey FR, Kempen GI, de Vugt ME: Qualitative study on needs and wishes of early-stage dementia caregivers: The paradox between needing and accepting help. Int Psychogeriatr 2015, 27(6):927-936.

21. Brodaty $\mathrm{H}$, Thomson $\mathrm{C}$, Thompson $\mathrm{C}$, Fine M: Why caregivers of people with dementia and memory loss don't use services. Int J Geriatr Psychiatry 2005, 20(6):537-546.

22. MacKenzie J: Stigma and dementia: East european and south asian family carers negotiating stigma in the uk. Dementia 2006, 5:233-247.

23. Shim B, Barroso J, Davis LL: A comparative qualitative analysis of stories of spousal caregivers of people with dementia: Negative, ambivalent, and positive experiences. Int J Nurs Stud 2012, 49(2):220-229. 
24. Banningh LW, Vernooij-Dassen MJ, Vullings M, Prins JB, Rikkert MG, Kessels RP: Learning to live with a loved one with mild cognitive impairment: Effectiveness of a waiting list controlled trial of a group intervention on significant others' sense of competence and well-being. Am J Alzheimers Dis Other Demen 2013, 28(3):228-238.

25. de Vugt ME, Verhey FR: The impact of early dementia diagnosis and intervention on informal caregivers. Prog Neurobiol 2013, 110:54-62.

26. Kjallman Alm A, Hellzen O, Norbergh KG: Experiences of long term ongoing structured support in early stage of dementia - a case study. Int J Older People Nurs 2013.

27. Craig P, Dieppe P, Macintyre S, Michie S, Nazareth I, Petticrew M, Medical Research Council G: Developing and evaluating complex interventions: The new medical research council guidance. Bmj 2008, 337:a1655.

28. Boots LM, de Vugt ME, Withagen HE, Kempen GI, Verhey FR: Development and initial evaluation of the web-based self-management program "partner in balance" for family caregivers of people with early stage dementia: An exploratory mixed-methods study. JMIR research protocols 2016, 5(1):e33.

29. Leontjevas R, Gerritsen DL, Koopmans RT, Smalbrugge M, Vernooij-Dassen MJ: Process evaluation to explore internal and external validity of the "act in case of depression" care program in nursing homes. J Am Med Dir Assoc 2012, 13(5):488 e481-488.

30. Morris JC: Clinical dementia rating: A reliable and valid diagnostic and staging measure for dementia of the alzheimer type. Int Psychogeriatr 1997, 9 Suppl 1:173-176; discussion 177-178.

31. Miller WR, Rollnick S: Motivational interviewing: Preparing people for change, second edition edn. New York: The Guilford Press; 2002.

32. Daniels R, van Rossum E, Metzelthin S, Sipers W, Habets H, Hobma S, van den Heuvel W, de Witte L: A disability prevention programme for communitydwelling frail older persons. Clin Rehabil 2011, 25(11):963-974.

33. Lorig $\mathrm{KR}$, Holman $\mathrm{H}$ : Self-management education: History, definition, outcomes, and mechanisms. Ann Behav Med 2003, 26(1):1-7.

34. Fortinsky $\mathrm{RH}$, Kercher K, Burant $\mathrm{CJ}$ : Measurement and correlates of family caregiver self-efficacy for managing dementia. Aging Ment Health 2002, 6(2):153-160. 
35. Lorig K, Chastain RL, Ung E, Shoor S, Holman HR: Development and evaluation of a scale to measure perceived self-efficacy in people with arthritis. Arthritis Rheum 1989, 32(1):37-44.

36. Radloff LS: The ces-d scale: A self-report depression scale for research in the general population. Applied Psychological Measurement 1977, 1:385-401.

37. Pinquart M, Sorensen S: Helping caregivers of persons with dementia: Which interventions work and how large are their effects? Int Psychogeriatr 2006, 18(4):577-595.

38. Kiresuk TJ, Sherman RE: Goal attainment scaling: A general method for evaluating comprehensive community mental health programs. Community Ment Health J 1968, 4(6):443-453.

39. Kiresuk TJ, Smith A, Cardillo JE: Goal attainment scaling: Applications, theory, and measurement, vol. xviii. Hillsdale, NJ, England: Lawrence Erlbaum Associates, Inc.; 1994.

40. Marson SM, Wei G, Wasserman D: A reliability analysis of goal attainment scaling (gas) weights. American Journal of Evaluation 2009, 30(2):203-216.

41. Pearlin LI, Schooler C: The structure of coping. J Health Soc Behav 1978, 19(1):2-21.

42. Kempen GIJM: Psychometric properties of glas baseline measures: A pilot study (in dutch). Northern Centre for Healthcare Research, University of Groningen, The Netherlands 1992.

43. Cohen S, Kamarck T, Mermelstein R: A global measure of perceived stress. J Health Soc Behav 1983, 24(4):385-396.

44. Andreou E, Alexopoulos EC, Lionis C, Varvogli L, Gnardellis C, Chrousos GP, Darviri C: Perceived stress scale: Reliability and validity study in greece. Int J Environ Res Public Health 2011, 8(8):3287-3298.

45. Zigmond AS, Snaith RP: The hospital anxiety and depression scale. Acta Psychiatr Scand 1983, 67(6):361-370.

46. Bjelland I, Dahl AA, Haug TT, Neckelmann D: The validity of the hospital anxiety and depression scale. An updated literature review. J Psychosom Res 2002, 52(2):69-77.

47. Grewal I, Lewis J, Flynn T, Brown J, Bond J, Coast J: Developing attributes for a generic quality of life measure for older people: Preferences or capabilities? Soc Sci Med 2006, 62(8):1891-1901. 
48. Makai P, Looman W, Adang E, Melis R, Stolk E, Fabbricotti I: Costeffectiveness of integrated care in frail elderly using the icecap-o and eq-5d: Does choice of instrument matter? Eur J Health Econ 2015, 16(4):437-450.

49. Lawrence RH, Tennstedt SL, Assmann SF: Quality of the caregiver--care recipient relationship: Does it offset negative consequences of caregiving for family caregivers? Psychol Aging 1998, 13(1):150-158.

50. de Vugt ME, Stevens F, Aalten P, Lousberg R, Jaspers N, Winkens I, Jolles J, Verhey FR: Behavioural disturbances in dementia patients and quality of the marital relationship. Int J Geriatr Psychiatry 2003, 18(2):149-154.

51. Hoekstra HA, Ormel J, De Fruyt F: Handleiding bij de neo persoonlijkheids vragenlijsten neo-pi-r en neo-ffi [manual for the neo personality inventories neo-pi-r and neo-ffi]. Lisse: Swets \& Zeitlinger; 1996.

52. Costa PT, McCrae RR: Neo pi-r: Professional manual. Odessa, FL: Psychological Assessment Resources; 1992.

53. Hakkaart- van Roijen L, Tan SS, Bouwmans CAM: Handleiding voor kostenonderzoek. Methoden en standaard kostprijzen voor economische evaluaties in de gezondheidszorg. Geactualiseerde versie 2010 [dutch manual for costing in economic evaluations]. Diemen: College voor zorgverzekeringen (CVZ); 2011.

54. Wimo A, Winblad B, Stoffler A, Wirth Y, Mobius HJ: Resource utilisation and cost analysis of memantine in patients with moderate to severe alzheimer's disease. Pharmacoeconomics 2003, 21(5):327-340.

55. Beauchamp N, Irvine AB, Seeley J, Johnson B: Worksite-based internet multimedia program for family caregivers of persons with dementia. Gerontologist 2005, 45(6):793-801.

56. Garson GD: Missing values analysis and data imputation. In. North Carolina State University: Statistical Associates Publishers; 2015.

57. van Gemert-Pijnen JE, Nijland N, van Limburg M, Ossebaard HC, Kelders SM, Eysenbach G, Seydel ER: A holistic framework to improve the uptake and impact of ehealth technologies. J Med Internet Res 2011, 13(4):e111.

58. Bouwens SF, van Heugten CM, Verhey FR: The practical use of goal attainment scaling for people with acquired brain injury who receive cognitive rehabilitation. Clin Rehabil 2009, 23(4):310-320.

59. Moore GF, Audrey S, Barker $\mathrm{M}$ et al: Process evaluation of complex interventions: Medical research council guidance. Bmj 2015, 350:h1258. 
60. Greene JC, Caracelli VJ, Graham WF: Toward a conceptual framework for mixed-method evaluation designs. Educational Evaluation and policy analysis 1989, 11(3):255-274.

61. Campbell M, Fitzpatrick R, Haines A, Kinmonth AL, Sandercock $P$, Spiegelhalter D, Tyrer P: Framework for design and evaluation of complex interventions to improve health. Bmj 2000, 321(7262):694-696. 


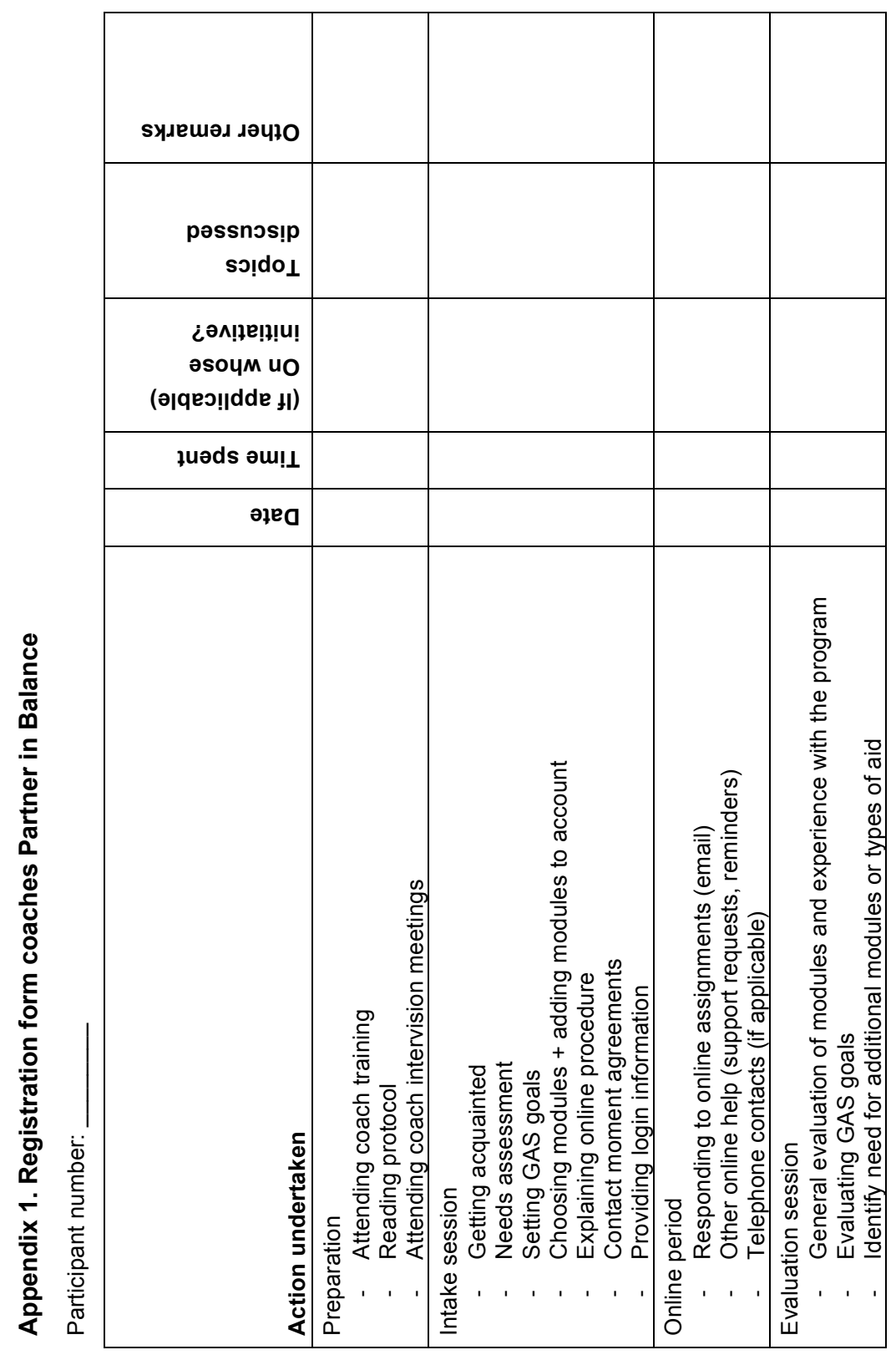





\section{CHAPTER 7}

Validity and implementation of the blended care selfmanagement program 'Partner in Balance' for caregivers of people with early-stage dementia: a process evaluation

Lizzy M.M. Boots, Marjolein E. de Vugt, Claudia M.J. Smeets, Gertrudis I.J.M. Kempen, Frans R.J. Verhey 


\section{ABSTRACT}

Background: The blended care self-management program 'Partner in Balance' (PiB) for family caregivers was executed in Dutch dementia care organizations.

Objective: A process evaluation was performed to determine internal and external validity assessing $\mathrm{PiB}$ credibility and generalizability of the results.

Methods: Implementation, sampling, and intervention quality were evaluated with quantitative and qualitative data from (1) coach questionnaires ( $N=13),(2)$ interviews with coaches $(\mathrm{N}=10)$ and participants $(\mathrm{N}=49)$, and (3) logistical research data. Goal attainment scaling (GAS) was used to measure treatment-induced change. Analyses were performed with descriptive statistics and deductive content analysis.

Results: The participation rate of caregivers was $52 \%$. Recruitment barriers were no computer and no need for support. Recruitment facilitators were young age and being employed. Caregivers were recruited by clinicians $(N=122)$; the Dutch Alzheimer Association (N=26); family members $(\mathrm{N}=4)$, or magazines and newspapers $(\mathrm{N}=11)$. Coaches $(\mathrm{N}=13)$ had a professional background as psychologist $(\mathrm{N}=7)$ or psychiatric nurse $(\mathrm{N}=6)$. All coaches received training and supervision in blended care self-management. Coaches reported that PiB deepened the contact with the caregiver. Caregivers appreciated the personalization and confirmation. The blended structure increased their openness. The program's primary focus on the caregiver and the discussion forum were appreciated less. Overall, goals were attained after the program ( $T>50)$. Performance according to protocol was substantial $(77 \%)$. Deviations were reported on intervention time, structure and feedback. Implementation barriers were lack of financing, time and deviating target population.

Conclusion: Caregivers and coaches were satisfied with the intervention, but the content should be adapted to younger caregivers. It could be considered to take age of the caregiver and background of the coach into account in the effect analysis. Awareness of benefits of blended care self-management and training is required for sufficient program implementation. 


\section{INTRODUCTION}

Caring for a family member with dementia puts one at risk for depression, anxiety and other health problems. ${ }^{1}$ The benefits of e-health support for dementia caregivers are increasingly recognized in dementia care practice. Multicomponent, tailored, interventions are particularly promising for increasing caregiver confidence and selfefficacy, and decreasing caregiver stress and depression. ${ }^{2}$ Blending online and faceto-face support increases caregiver-therapist relations and adherence. ${ }^{3}$ Dementia caregiver support is well-established, ${ }^{4}$ although supporting caregivers early in the dementia process is not commonplace but could prevent high levels of burden and psychological problems in the later stages. ${ }^{5}$ Early stage interventions should not be problematizing, but should meet the challenges and needs of the early phases. Existing programs are mostly aimed at problem-behaviors that occur in the advanced stages of dementia. ${ }^{6-11}$ To this end, the blended care program 'Partner in Balance' for caregivers in the early stages was developed, aimed at the enhancement of the positive, intact experiences, tailored to the individual caregiver. ${ }^{12}$ To evaluate the program's effectiveness on caregiver self-efficacy, mastery, and psychological complaints, a randomized controlled trial (RCT) was performed. Many caregiver ehealth support studies lack methodological rigor, ${ }^{2}$ and RCTs are considered proper designs for convincing evidence. But even with a prudent study design, evaluation of sampling quality and intervention quality is essential for the interpretation and generalizability of results. ${ }^{13}$ Furthermore, knowledge on implementation barriers and facilitators in an effect study can aid future adoption of a new intervention in care practice. Generalization and applicability of findings (external validity) is especially of interest for clinicians and policymakers. A newly developed program can be very effective, but difficult to implement in care practice if health professionals or policy makers do not accept it. Implementation can be complicated in the complex and heterogeneous structure and culture of dementia care organizations. ${ }^{14}$ Therefore, process evaluations should focus on internal validity and implementation knowledge to increase credibility. ${ }^{13}$ Different frameworks for process evaluations have been proposed, but there is no consensus on elements that process evaluations should cover ${ }^{13}$. In this study we describe first order (sampling and intervention quality) and second order process data (implementation knowledge), based on the model of Leontjevas et al., ${ }^{13}$ to assess the internal and external validity of the PiB trial and its implementation. 


\section{METHODS}

The process evaluation was conducted alongside the effectiveness study. Detailed information on the methods are presented in Chapter $6 .{ }^{15} \mathrm{~A}$ short description is provided below.

\section{Study design}

A randomized waiting-list controlled trial was conducted in the south of the Netherlands. Family caregivers were recruited within memory clinics, mental health care organizations and caregiver support services. The intervention was delivered and evaluated within these organizations. A waiting-list controlled design was chosen to increase acceptability and adherence to the research protocol in the control group, and decrease attrition effects. ${ }^{16}$ Data were collected pre- and post-intervention and at 3,6 and 12 months follow-up.

\section{Intervention}

Detailed information about the program components and development is presented in Chapter $5^{12}$ and program results will be presented in Chapter 8 . In short, the blended care self-management program 'Partner in Balance' (PiB) consists of (1) a face-to-face intake session with the personal coach to familiarize with the coach and program, set goals with the Goal Attainment Scaling method ${ }^{17}$ and select relevant modules, (2) engagement in four out of nine available online thematic modules including psycho-education, behavioral modeling, reflective assignments, change plans and e-mail feedback from the coach during 8 weeks, and (3) a face-to-face evaluation session with the coach in which previously set goals are assessed. Furthermore, participants can exchange information with other participants via the discussion forum. Module themes are Acceptance, Balance in activities, Communication with family member and environment, Coping with stress, Focusing on the positive, Insecurities and rumination, Self-understanding, The changing family member, and Social relations and support. Two weeks were allocated per module, but participants were allowed to complete the modules at their own pace in accordance with the self-management approach. ${ }^{18}$ The personal page and modules remained accessible for participants after the intervention period. Participants in the control group were on a waiting list for 8 weeks, while receiving usual care (nonfrequent counseling). After the post-test assessment they were given the opportunity to follow PiB. 
The personal coach

The personal coaches were trained, experienced professionals (psychologist or psychiatric nurse) from one of the participating organizations. They received a 2-hour training in self-management techniques, goal setting and online help, and regular supervision meetings. Their tasks were familiarizing participants with the online program, supporting them in module choice and goal setting, and giving feedback on online self-reflective assignments.

\section{Process evaluation}

First-order process data were evaluated prior to the effect analysis. Second-order process data are presented together with the first order data for a complete overview of the implementation and trial quality. ${ }^{14}$ The framework for this process evaluation was based on earlier research. ${ }^{13,14}$

\section{First order process data}

The sampling quality was determined by a description of the procedures of recruitment, informed consent, allocation, recruitment barriers and facilitators, and reach. Data were extracted from the research database and 2 focus group interviews with 5 coaches per group (See Box 1 for topics). The intervention quality (relevance, feasibility, and performance according to protocol) was determined on two levels; (1) coaches, and (2) participants.

First-level data collection (coaches) involved registration of protocol deviations and amount and intensity of contact with caregivers on a structured registration form (see Appendix I of the previous Chapter 6), an 8-item questionnaire rating the overall usability of $\mathrm{PiB}$ and its relevance for caregivers and coaches with 4 multiple-choice items rated on a 5 -point scale ( $1=$ completely disagree to $5=$ completely agree) (see Appendix I of the current Chapter), and 4 open-ended items on the advantages, disadvantages, recommendation to other organizations or colleagues, and general appreciation of the program.

Second-level data (participants) were collected post-intervention by a semistructured interview with participants regarding their experiences with $\mathrm{PiB}$ (Box 1). Furthermore, Goal attainment scaling $(G A S)^{17}$ was used to measure treatmentinduced change and to compare relative success of previously set personal goals. GAS is a suitable measure to translate goals into achievement ratings. The scores range from -2 (much less than expected) to +2 (much better than expected), with a 
score of 0 meaning that the goal was attained. Usage of the website (clickstream per intervention component) was tracked. Clickstream data are information-trails users leave behind while visiting the website captured in a log file. Clicks represent the number of times a page has been viewed. ${ }^{19}$

Box 1. Topic list for interviews with participants and coaches

\begin{tabular}{|c|c|}
\hline TOF & \\
\hline INDIVIDUAL INTERVIEW PARTICIPANTS & FOCUS GROUP INTERVIEW COACHES \\
\hline $\begin{array}{l}\text { - } \text { Clarity of content } \\
\text { - } \quad \text { Sebsite ease of use } \\
\text { - } \text { face-to-face) } \\
\text { - Satisfaction personal coach and } \\
\text { - } \text { feedback } \\
\text { - } \text { the pronantages and disadvantages of } \\
\text { Recommendations }\end{array}$ & $\begin{array}{l}\text { - Feasibility: recruitment, instructions, time } \\
\text { investment, collaboration researchers } \\
\text { - Value for participating caregivers } \\
\text { - Barriers and facilitators for program } \\
\text { implementation }\end{array}$ \\
\hline
\end{tabular}

\section{Second-order process data}

Knowledge on implementation of the program (components delivered and received, and barriers and facilitators to implementing) was investigated with additional items in the questionnaire for coaches described above ( 2 open-ended items on barriers and facilitators for program implementation, see Appendix I), and the data obtained from the focus group interviews (Box 1).

\section{Analysis}

Descriptive statistics (SPSS version 22.0) were used for the quantitative data analysis. Interviews were audiotaped and transcribed verbatim. Deductive content analysis was used for the qualitative data analysis (open-ended questions and transcripts) by authors LMMB and CMJS, using the qualitative analysis software ATLAS.ti (Version 1.0.14 for Macintosh). Topics that were mentioned frequently and explicitly served as the basis for the categorization. Categories were merged into common themes in a consensus meeting (LMMB and CMJS). Raw GAS scores were transformed into an individual mean GAS score (T-score) to determine goal attainment. ${ }^{17}$ T-scores included attainment level and a potential weight assigned to the goal(s). T-scores of $\geq 50$ indicate effective goal achievement. 


\section{RESULTS}

\section{First-order process data}

\section{Sampling quality}

Recruitment and randomization

Family caregivers $(\mathrm{N}=163)$ were recruited from memory clinics (MUMC+, Elkerliek Hospital Helmond, Catharina Hospital Eindhoven), ambulatory mental health clinics (Virenze-RIAGG Maastricht, MET ggz Roermond), caregiver support services in the southern regions of the Netherlands, and the Dutch Alzheimer Association. If interested, family caregivers $(\mathrm{N}=138)$ received the detailed information letter. Reasons for declining more information were not meeting the inclusion criteria at double check $(\mathrm{N}=9)$, no need for support $(\mathrm{N}=6)$, no computer/experience $(\mathrm{N}=5)$, too busy $(\mathrm{N}=2)$, care recipient does not agree with caregiver participation $(\mathrm{N}=1)$, family emergency $(\mathrm{N}=1)$, or unknown $(\mathrm{N}=1)$. Of the information recipients, informed consent (IC) was signed by $58 \%(80 / 138)$. Reasons for not signing the IC after more detailed information were, no need for support $(\mathrm{N}=8)$, no computer/experience $(\mathrm{N}=8)$, too busy $(\mathrm{N}=12)$, health problems $(\mathrm{N}=6)$, care recipient does not agree with caregiver participation ( $\mathrm{N}=4)$, personal contact preferred over e-health $(\mathrm{N}=8)$, participation feels like a burden $(\mathrm{N}=9)$, or unknown $(\mathrm{N}=3)$. Of the 163 caregivers 154 were eligible. The participation rate of eligible caregivers was $52 \%$ (80/154).

Following the baseline assessment, 80 participants were randomly allocated to either Partner in Balance or the waiting-list control group. The researcher (LMMB) not involved in the assessments performed the allocation. A research assistant blind to allocation conducted the assessments, and recorded blinding success and reason for possible unmasking on the Case Record Form. At T1, 68 participants had completed the post-intervention or post-waiting list assessment and blinding was intact for $46 \%$ (31/68), unsuccessful for $49 \%(33 / 68)$, and for $7 \%(5 / 68)$ a conjecture of allocation was expressed.

\section{Barriers and facilitators for recruitment}

The interviewed coaches $(\mathrm{N}=10)$ reported that their caseload comprised many people with dementia living alone without a registered primary caregiver. This was listed as a primary recruitment barrier, next to "caregiver does not own computer" and "caseload only comprises caregivers of people with moderate to severe dementia". Other barriers included concerns of burden for caregivers, high staff workload, and involvement in other caregiver support approaches. Coaches reported 
that younger caregiver age and being employed were facilitators for program recruitment, as e-health suits a busy schedule and work-related computer literacy.

Reach

Caregivers were invited to participate by the clinician who treated their family member $(\mathrm{N}=122)$; informed about the program's existence by the Dutch Alzheimer Association $(\mathrm{N}=26)$; or knew caregivers/family members already involved in the program $(\mathrm{N}=4)$. Others $(\mathrm{N}=11)$ requested information based on editorials in health magazines, local newspapers and information stands in the south of the Netherlands. The Dutch Alzheimer Association disseminated information about the program via (1) monthly meeting spots for people with dementia and their caregivers, (2) newsletters, (3) their website and social media platforms Facebook and Twitter.

\section{Intervention quality}

Relevance and feasibility

Coaches. All coaches $(\mathrm{N}=13)$ completed the questionnaire and rated 'Partner in Balance' as adequately feasible in daily practice (M 4.5, SD 0.5) and fairly easy to integrate in their work-related activities (M 4.4, SD 0.5). The program was rated as a useful addition for family caregivers (M 4.5, SD 0.5), and for the coach as a professional caregiver (M 4.2, SD 0.8). The mean time spent per participant was 6.2 hours (SD 1.5) spread over 8 weeks: intake session 1.9 hours (SD 0.5), online feedback 2.5 hours (SD 1.0), evaluation session 1.4 hours (SD 0.7). Categories emerged from the open-ended questions in the questionnaire and the focus group interviews were divided into two themes: advantages for coaches and caregivers and experienced difficulties and suggestions for improvement. Themes are described below and illustrated with quotations. Each quotation is assigned a code indicating the coach number in the trial.

\section{Advantages for coaches and caregivers}

The detailed situations described by participants enabled coaches to empathize with them and focus their feedback. They reported a more profound relationship with the participant after the intervention. The flexibility to provide feedback whenever and wherever was considered very positive; it fitted their busy schedule and offered them the time to reflect on their words. The face-to-face contact during the intake session was necessary to create a personal connection with the participant. Coaches 
stressed that the examples offered recognition for participants, and the assignments increased awareness about behaviors and feelings. The switch from thinking in problems to thinking in quantifiable goals was considered very valuable. The availability of the information, assignments and feedback after the intervention was seen as an advantage over merely face-to-face support.

"Analyzing problems makes them easier to solve. With the focus on a particular problem, other issues are raised that normally would not be addressed. It really breaks the normal routine for both client and coach. I noticed it deepens the relationship you have with people." [C1]

\section{Experienced difficulties and suggestions for improvement}

Some coaches struggled with their level of direction in the self-management approach, as they were accustomed to a pro-active care role and felt the urge to assist more than instructed for this PiB program. Goal setting during the intake session was considered difficult by the coaches. However, they stressed the importance of goal setting and quantifying possible outcomes of program participation to make them tangible.

Several suggestions for improvement were mentioned. The user-friendliness of the instant messaging could be increased. Furthermore, a possible alarm function for crisis situations and follow-up care options were desired. To avoid confusion in module structure, it was suggested to make modules available to caregivers one by one, not all at once.

Participants. Participants who completed the intervention ( $N=49)$ most often selected the available themes communication with family member and environment $(44 \mathrm{x})$ and self-understanding (44x), followed by the changing family member (39x), acceptance (36x), coping with stress (30x), balance in activities (24x), insecurities and rumination $(21 \mathrm{x})$, focusing on the positive $(20 \mathrm{x})$, and social relations and support (20x). Emerged categories from the semi-structured interviews were divided into four themes: Program content, program structure, role of the personal coach, and target audience. Each quotation is assigned a code indicating the participant number in the trial. 


\section{Program content}

Most participants were satisfied with the content, as it suited their current concerns. Specifically the personalized assignments and challenges were appreciated. It was often mentioned that the content was not innovative, but a confirmation of participants' conjectures. Some participants struggled with the primary focus on the caregiver, as they felt the care recipient should change.

"I think it's odd that the caregiver has to make changes, and my wife [person with dementia] doesn't have to learn or change anything" [P25]

Suggestions for content improvement were also made. Including links to more disease-specific information was desired. Adding subtitles to the movie clips to watch them on mute was preferred when the care recipient was not aware of the caregiver's participation.

\section{Program structure}

The program structure of blending face-to-face contacts with online modules was experienced positively. Participants mentioned that the face-to-face contact personalized the program and increased their openness during the online assignments.

"You need to see who is going to ask you these intimate questions. In my opinion that is a prerequisite for sending such personal information." [P68]

It was stressed that the module structure facilitated the personal assignments; the examples fostered reflection and the tips were used as input. Others reported difficulties compartmentalizing their answers in the assignment structure. Participants mentioned using the website when they had time and often revisited the examples, their own answers or the feedback. A printed workbook and an autosave option were considered desirable additions.

Role of the personal coach

The feedback was considered supportive and critical at times, allowing participants to reflect on their answers. Participants emphasized that the coach was essential for 
motivation and questions. Knowing that there was someone there to guide them, made them feel less alone.

"She [coach] made me think about things. You don't expect the feedback to be like the philosopher's stone, but it's nice to get some confirmation and sometimes a critical note; you are saying this, but how are you going to live up to it? You're not doing it alone" [P03]

Participants with a familiar coach reported an intensified relationship postintervention, by being able to speak freely online and getting acquainted with the coach on a different level. Lastly, participants mentioned coaches should not participate on the discussion forum, as this was a safe zone for caregivers' opinions.

\section{Target audience}

Younger participants felt that the program should be specified to different subgroups. They could not identify with the older population in the examples, as they were still employed and dealt with other issues in daily life, such as (young) children living at home. Furthermore, caregivers of people with young-onset dementia missed the behavioral disturbances in the examples, as this was something they struggled with daily.

"As a younger and employed person I cannot identify with the older people and their struggles in the movie clips. It makes me feel alone." [P67]

The forum was not used, as caregivers struggled with shame in the early stages and sharing their story felt like betrayal to the care recipient. Reading about other people's misery was considered undesirable. Some participants mentioned that the course came too late for them. It was stressed that the program should be made available for all caregivers following diagnostic disclosure.

"I was exceptionally alert and active in my search for information, but it should be provided to anyone. Don't wait until people ask for it. I considered it an 'integration course' for caregivers" [P54] 


\section{Goal attainment}

Of the program completers in both groups $(\mathrm{N}=49), 42$ participants set 93 goals in total. Five program completers were not able to set goals as they felt their care recipient should change and two completers had no goals due to coach turnover. The majority of participants $(\mathrm{N}=35)$ achieved $\geq 1$ goal(s). Overall, seventy goals were attained (43 attained, 17 higher than expected, 10 much higher than expected), and 22 goals were not attained (17 lower than expected, 5 much lower than expected). The mean T-score at baseline (set at -2 level) was 25.2 (SD 3.4). The mean achieved T-score at post-intervention was 50.1 (SD 10.08). Table 1 shows the number of goals for each domain, with most goals set on communication with the care recipient, followed by planning time alone and not feeling guilty about it, decreasing or preventing tension and anxiety, and obtaining social support.

Table 1. Number of set goals, attainment scores and domains $(\mathrm{N}=42)$

\begin{tabular}{lll}
\hline & Mean (SD) & Range \\
\hline Number of set goals per participant & $2.2(1.1)$ & $1.0-4.0$ \\
\hline$G A S^{a}$ score at baseline & $25.5(3.4)$ & $21.0-30.0$ \\
\hline$G A S^{a}$ score achieved & $50.1(10.8)$ & $30.0-77.4$ \\
\hline Number of set goals per domain $(N)$ & & \\
- Communication with care recipient & 21 \\
- Positive activities with care recipient & 5 \\
- Social support and contacts & 10 \\
- Time alone and feeling guilty & 16 \\
- Tension and anxiety & 13 \\
- $\quad$ Role and relationship changes & 9 \\
- Feeling in control by gaining knowledge & 8 \\
- $\quad$ Positive thoughts and rumination & 11 \\
\hline
\end{tabular}

${ }^{\mathrm{a}} \mathrm{GAS}=$ Goal Attainment Scaling

\section{Performance according to protocol}

Coaches. Intervention performance according to protocol comprised: face-to-face intake session, online modules during 8 weeks, individualized feedback via email per module, and a face-to-face evaluation session. Ten coaches reported performance according to protocol (77\%), three reported deviations in intervention time, structure and feedback $(23 \%)$. Intervention time was reported longer $(\mathrm{N}=2)$ or shorter $(\mathrm{N}=1)$ than 8 weeks, module structure was consumed differently than intended $(\mathrm{N}=2)$ or feedback was given by telephone $(\mathrm{N}=2)$ or face-to-face $(\mathrm{N}=1)$. Reasons to deviate 
from the protocol were caregiver pace and understanding of the program structure, illness, holiday leave, changes in work load and hours, personal family emergencies, and struggling to verbalize feedback in an email.

Participants. With regard to dose delivered, of the program completers $(\mathrm{N}=49)$ $87.8 \%$ (43/49) completed all 4 modules, 6.1\% (3/49) 3 modules, and $6.1 \%(3 / 49) 2$ modules. The tracked usage data showed 21946 clicks per module, including scoping the website (2444 clicks), viewing the psycho-education (3922 clicks) completing the assignments and change plan (8748 clicks), contacting the personal coach (6489 clicks) and visiting the discussion forum (310 clicks). Total intervention time ranged from 4 to 32 weeks (M 13.9, SD 6.8). Reasons for intervention period variance were holiday, illness, busy schedules, and technical difficulties. Following the intervention period, $77.6 \%$ of the program completers (38/49) requested access to the additional modules, with (16/49) or without (33/49) the coach at their disposal for questions.

\section{Second-order process data}

Implementation components

Coaches $(\mathrm{N}=13)$ had a professional background as psychologist $(\mathrm{N}=7)$ or psychiatric nurse $(\mathrm{N}=6)$. During the regular supervision meetings coaches shared experiences and asked feedback. Support concerning the website and feedback content was requested outside the supervision meetings via e-mail $(\mathrm{N}=13)$ and telephone $(\mathrm{N}=6)$ during the trial.

\section{Barriers and facilitators for implementation}

Directors of 22 dementia care organizations in the southeast of the Netherlands were asked to participate in the trial. The response rate of organizations was $73 \%(16 / 22)$. Of the responders, $63 \%(10 / 16)$ expressed their interest to adopt the program. Refusal was based on upcoming reorganization (1/6), no suitable caregivers in caseload (4/6), or high workload of staff members (1/6). Of the interested organizations, $40 \%$ (4/10) choose to implement 'Partner in Balance' and train staff members (psychologists or psychiatric nurses) to act as personal coaches. Six organizations $(60 \%)$ choose to refer caregivers to the coordinating center, due to high staff workload. Emerged categories regarding implementation barriers and facilitators from the open-ended questions and focus groups were divided into three themes: organizational aspects and financing, time and practical aspects, and 
organization's target population. Themes are described below and illustrated with quotations followed by a code indicating the coach number in the trial.

\section{Organizational aspects and financing}

Coaches mentioned that elderly care organizations in the Netherlands often file caregiver support under patient care, which could create problems for implementation of caregiver support programs when the person with dementia is not registered. Also, financial cutbacks hampered professionals in adopting new support tools. Mentioned facilitators were separate registration of caregiver support, insurance compensation, and integration of online support in already provided caregiver support.

"If we cannot register caregiver support, we cannot make time for it. Managers need to be convinced that caregiver support also benefits the person with dementia" [C3]

Time and practical aspects

It was mentioned that unfamiliarity with the program could create a barrier for future implementation. The training and self-study was considered a substantial personal time investment. Coaches suggested training all staff members as coach and applying the program as regular care to facilitate implementation.

“We don't get a lot of time per client so taking too much time for training feels wrong." [C9]

"The program could easily be implemented as regular care if all staff members or a constant group of staff members were trained as coach." [C4]

\section{Organization's target population}

Professionals mentioned that many health care organizations only treat people with moderate to severe dementia with severe comorbidity, or they expect the family caregivers to not be computer literature due to their high age, which hampers implementation of this type of caregiver support. 


\section{DISCUSSION}

This study described the process of Partner in Balance $(\mathrm{PiB})$ to explore its credibility and generalizability. First-order and second-order process data were evaluated from the perspective of both family caregivers (participants) and professionals delivering the intervention (coaches), increasing the understanding of the mode of delivery. ${ }^{20}$

\section{Sampling and randomization}

Data on sampling quality showed that the participation rate of caregivers was $52 \%$. Considering an average response rate of $27 \%$ for caregiver research, our participation rate can be considered substantial. ${ }^{21}$ However, the $52 \%$ only considered eligible caregivers who where already familiar with the care parties involved in recruitment and may have be more motivated and open to support. ${ }^{22}$ Recruitment barriers were e.g. no computer, no need for intervention or additional support, which were also reasons to decline participation. During the early stages, caregivers may struggle with a fear of stigma, shame and low acceptance. ${ }^{23}$ This might explain why participants were not ready to openly discuss their situation on the discussion forum and why some participants struggled with acceptance of the situation, as was illustrated by stating that their care recipient should change. Young age and being employed were considered recruitment facilitators. However, caregivers of people with young-onset dementia and children had difficulties identifying with the older caregivers in the examples. Previous research confirmed that younger caregivers struggle with different aspects in daily life, when compared to older caregivers ${ }^{24}$. It is essential that to match program content and specific needs of the target audience to maximize the benefit of a supportive intervention. ${ }^{2,25-27}$ The thematic structure of the program allows for add-on of specific themes for subgroups, such as caregivers of people with young-onset dementia.

At post-intervention assessment $(\mathrm{N}=68)$, blinding of allocation was intact for only $46 \%$ (31/68) of the participants, which can potentially bias the estimation of effectiveness. However, blinding in psychosocial research can be challenging and it is rarely reported if blinding is maintained. ${ }^{28}$ This item from the Consolidated Standards of Reporting Trials (CONSORT) may have been developed with studies of medical treatments in mind, but blinding or masking may be unfeasible for certain aspects of psychosocial treatment studies. ${ }^{29}$ Some other important aspects of psychosocial RCTs need to be considered and reported but are not addressed in the CONSORT statement, e.g. adequate reporting of treatment integrity (whether 
therapists were consistent in providing the specified intervention). Verification of treatment integrity, or fidelity, in outcome studies is essential to ensure that valid comparisons of replicable treatments can be made. ${ }^{30}$

\section{Intervention quality}

Professionals were satisfied with the intervention fitting their busy schedule, giving them time to focus their feedback. They reported a more profound relationship with the caregiver, as the program allowed them to empathize with the caregiver. However, the low level of professional involvement in the self-management approach was considered a challenge. This issue deserves further exploration, as the performance of the self-management health care provider is very important for the participant's performance of self-management tasks and overall intervention effectiveness. ${ }^{31}$ It was previously argued that health care providers do not always support self-management education, as this is not part of their attitudes, beliefs or regular care practice. ${ }^{18}$ To maximize the effects of self-management programs, it is recommended to increase the essential clinical competences of healthcare providers to provide tailored self-management support, ${ }^{27}$ and that the health care system increases its awareness of the benefits of evidence-based self-management programs for their target population. ${ }^{18}$ For one, the present study showed that both participants and coaches mentioned that their relationship with each other had deepened, which was also demonstrated in a previous blended-care intervention for depression. ${ }^{32}$ Previous research confirmed that the opportunity to reflect on one's feelings anonymously in one's personal safe environment is easier than face-to-face, but the face-to-face contact increased caregiver openness, and therefore coach empathy with their situation. ${ }^{3}$ Participant compliance to all 4 modules was high $(87.8 \%)$, which could be explained by motivational aspect of having a coach, also mentioned in previous studies. ${ }^{3}$ The varying intervention period and dose may influence the effectiveness of the intervention. ${ }^{30}$ However, reasons for protocol deviations were diverse and not uncommon for informal caregivers and elderly participants (e.g. caregiver pace, time constraints, illness). ${ }^{21}$ In self-management interventions the participant is in control and should therefore be allowed to complete the modules at his own pace. ${ }^{18,33}$ Furthermore, complex interventions such as PiB are often designed to be implemented with some flexibility, in order to accommodate differences across participants. ${ }^{34}$ 


\section{Implementation}

Pib was evaluated within multiple organizations with coaches from different backgrounds, who received training and supervision in self-management and blended care. A relatively high response rate of organizations was found $(73 \%)$, which could be attributed to the current demand of health care insurances for care organizations to provide e-health. ${ }^{35}$ However, a lack of financing and time could hamper the implementation. The MRC Framework suggests that implementation should be considered during the first phases of intervention development and evaluation. ${ }^{36}$ As complex interventions are influenced by context, many psychosocial interventions show different results in different settings or countries. ${ }^{29}$ Additional information about the process of the implementation is crucial to understanding why an intervention is effective in one setting and not in another. ${ }^{34} \mathrm{PiB}$ was evaluated within different settings with coaches of different backgrounds delivering the intervention. To evaluate if coach background has any influence on the intervention outcome, this variable will be considered in the effect analysis.

\section{Limitations and recommendations for future research}

In our study, protocol deviations were measured with the self-report questionnaire for coaches. Previous research measuring protocol deviations with self-report questionnaires and ratings based on tape-recordings found large discrepancies between the two measurements, indicating that professionals may not always be aware of their level of treatment fidelity. ${ }^{37}$ Furthermore, tracked usage data were measured in clicks. Clicks represent page views, but this clickstream method has a large disadvantage; many people who click on a page do not necessarily read it. Even one in three visitors spend less than 15 seconds reading the page, so a measured click does not automatically mean the attention of the visitor is directed to the information on the page that is being viewed. ${ }^{19}$ Nevertheless, the results showed that the participants spent most of their time on the assignments and change plans and the e-mail contact with their coach, which represent the essential elements of a blended-care program. ${ }^{3,32}$

Goal attainment scaling (GAS) was used to rate goal attainment. Goal setting and rating is considered a therapeutic task and was therefore performed by the coaches during the face-to-face sessions. Future research could consider setting and rating goals by an independent research assistant in all treatment arms to consider GAS as an outcome measure. However, GAS is a challenging evaluation method 
when not all participants set or evaluate goals, goals change along the way, or participants lack insight, communicational skills or the capacity to specify goals. Therefore, previous research recommends using GAS as a complementary scale, not as a replacement of other outcome instruments. ${ }^{38}$

\section{Conclusion}

Participants and professionals were satisfied with the intervention, but the content should be adapted to specific subgroups such as younger caregivers. It could be considered to take age of the caregiver and background of the coach into account in the effect analysis. Implementation of the program requires more awareness of the benefits of blended care self-management programs and training in tailored selfmanagement skills for the healthcare provider. Overall, Partner in Balance can be considered a valuable addition to the already provided caregiver support, as it is tailored to the needs of the target audience and deepened the coach-caregiver relationship. GAS enables a clinical evaluation of individual participants that benefit from the program. 


\section{References}

1. Brodaty H, Donkin M. Family caregivers of people with dementia. Dialogues Clin Neurosci 2009; 11(2): 217-28.

2. Boots LM, de Vugt ME, van Knippenberg RJ, Kempen GI, Verhey FR. A systematic review of Internet-based supportive interventions for caregivers of patients with dementia. Int J Geriatr Psychiatry 2014; 29(4): 331-44.

3. Wilhelmsen M, Lillevoll K, Risør MB, et al. Motivation to persist with internetbased cognitive behavioural treatment using blended care: a qualitative study. BMC Psychiatry 2013; 13(296).

4. Olazaran J, Reisberg B, Clare L, et al. Nonpharmacological therapies in Alzheimer's disease: a systematic review of efficacy. Dement Geriatr Cogn Disord 2010; 30(2): 161-78.

5. de Vugt ME, Verhey FR. The impact of early dementia diagnosis and intervention on informal caregivers. Prog Neurobiol 2013; 110: 54-62.

6. Hattink B, Meiland F, van der Roest $\mathrm{H}$, et al. Web-Based STAR E-Learning Course Increases Empathy and Understanding in Dementia Caregivers: Results from a Randomized Controlled Trial in the Netherlands and the United Kingdom. J Med Internet Res 2015; 17(10): e241.

7. Blom MM, Zarit SH, Groot Zwaaftink RB, Cuijpers P, Pot AM. Effectiveness of an Internet intervention for family caregivers of people with dementia: results of a randomized controlled trial. PLoS One 2015; 10(2): e0116622.

8. Cristancho-Lacroix V, Wrobel J, Cantegreil-Kallen I, Dub T, Rouquette A, Rigaud AS. A web-based psychoeducational program for informal caregivers of patients with Alzheimer's disease: a pilot randomized controlled trial. J Med Internet Res 2015; 17(5): e117.

9. Hayden LJ, Glynn SM, Hahn TJ, Randall F, Randolph E. The Use of Internet Technology for Psychoeducation and Support With Dementia Caregivers. Psychol Serv 2012; 9(2): 215-8.

10. Kajiyama B, Thompson LW, Eto-Iwase T, et al. Exploring the effectiveness of an internet-based program for reducing caregiver distress using the iCare Stress Management e-Training Program. Aging Ment Health 2013; 17(5): 54454.

11. van der Roest HG, Meiland FJM, Jonker C, Droes RM. User evaluation of the DEMentia-specific Digital Interactive Social Chart (DEM-DISC). A pilot study 
among informal carers on its impact, user friendliness and, usefulness. Aging Ment Health 2010; 14(4): 461-70.

12. Boots L, De vugt M, Withagen H, Kempen G, Verhey Fip. Development and initial evaluation of the web-based self-management intervention 'Partner in Balance' for family caregivers of people with early-stage dementia: an exploratory mixed-methods study. JMIR research protocols 2016.

13. Leontjevas R, Gerritsen DL, Koopmans RT, Smalbrugge M, Vernooij-Dassen MJ. Process evaluation to explore internal and external validity of the "Act in Case of Depression" care program in nursing homes. J Am Med Dir Assoc 2012; 13(5): 488 e1-8.

14. Zwijsen SA, Smalbrugge M, Eefsting JA, Gerritsen DL, Hertogh CM, Pot AM. Grip on challenging behavior: process evaluation of the implementation of a care program. Trials 2014; 15: 302.

15. Boots LMM, de Vugt ME, Kempen GIJM, Verhey FRJ. Effectiveness of the blended care self-management program 'Partner in Balance' for early-stage dementia caregivers: study protocol for a mixed-methods single-blind randomized waiting-list controlled trial. Trials (submitted) 2016.

16. Campbell $M$, Fitzpatrick $R$, Haines $A$, et al. Framework for design and evaluation of complex interventions to improve health. Bmj 2000; 321(7262): 694-6.

17. Kiresuk TJ, Smith A, Cardillo JE. Goal attainment scaling: Applications, theory, and measurement. Hillsdale, NJ, England: Lawrence Erlbaum Associates, Inc.; 1994.

18. Lorig KR, Holman H. Self-management education: history, definition, outcomes, and mechanisms. Ann Behav Med 2003; 26(1): 1-7.

19. Haile T. What you think you know about the web is wrongMarch 9, 2014, $2014 . \quad$ http://time.com/12933/what-you-think-you-know-about-the-web-iswrong/ (accessed February 25, 2016).

20. Haastregt JCMv. Preventing falls and mobility impairments in elderly people living in the community. Maastricht: Maastricht University; 2002.

21. AARP. Appendix A: Detailed Methodology. Washington D.C., 2004.

22. Brodaty $\mathrm{H}$, Thomson $\mathrm{C}$, Thompson $\mathrm{C}$, Fine M. Why caregivers of people with dementia and memory loss don't use services. Int J Geriatr Psychiatry 2005; 20(6): 537-46. 
23. Boots LM, Wolfs CA, Verhey FR, Kempen GI, de Vugt ME. Qualitative study on needs and wishes of early-stage dementia caregivers: the paradox between needing and accepting help. Int Psychogeriatr 2015; 27(6): 927-36.

24. Millenaar JK, de Vugt ME, Bakker C, et al. The Impact of Young Onset Dementia on Informal Caregivers Compared with Late Onset Dementia: Results from the NeedYD Study. Am J Geriatr Psychiatry 2015.

25. Brown RM, Brown SL. Informal caregiving: a reappraisal of effects on caregivers. Social Issues and Policy Review 2014; 8(1): 74-102.

26. Tang WK, Chan CY. Effects of psychosocial interventions on self-efficacy of dementia caregivers: a literature review. Int J Geriatr Psychiatry 2015.

27. Trappenburg J, Jonkman N, Jaarsma T, et al. Self-management: one size does not fit all. Patient Educ Couns 2013; 92(1): 134-7.

28. Mayo-Wilson E, Grant S, Hopewell S, Macdonald G, Moher D, Montgomery P. Developing a reporting guideline for social and psychological intervention trials. Trials 2013; 14: 242.

29. Ladd BO, McCrady BS, Manuel JK, Campbell W. Improving the quality of reporting alcohol outcome studies: effects of the CONSORT statement. Addictive behaviors 2010; 35(7): 660-6.

30. Moncher FJ, Prinz RJ. Treatment fidelity in outcome studies. Clinical Psychology Review 1991; 11(3): 247-66.

31. Greene JC, Yedidia MJ. Provider behaviors contributing to patient selfmanagement of chronic illness among underserved populations. Journal of Health Care for the Poor and Underserved 2005; 16(4): 808-24.

32. van der Vaart R, Witting M, Riper H, Kooistra L, Bohlmeijer ET, van GemertPijnen LJEWC. Blending online therapy into regular face-to-face therapy for depression: content, ratio and preconditions according to patients and therapists using a Delphi study. BMC Psychiatry 2014; 14(355).

33. Daniels $R$, van Rossum E, Metzelthin $S$, et al. A disability prevention programme for community-dwelling frail older persons. Clin Rehabil 2011; 25(11): 963-74.

34. Hawe P, Shiell A, Riley T. Complex interventions: how "out of control" can a randomised controlled trial be? Bmj 2004; 328(7455): 1561-3.

35. Baur A, Fehr J, Mayer C, Pawlu C, Schaudel F. Health care beyond medicine: meeting the demands for new forms of care. Health International, 2011. http://www.mckinsey.com/ /media/mckinsey/dotcom/client_service/pharma 
and medical products/pmp new/pdfs/healthcare_beyond_medicine.ashx (accessed.

36. Craig $\mathrm{P}$, Dieppe $\mathrm{P}$, Macintyre $\mathrm{S}$, et al. Developing and evaluating complex interventions: the new Medical Research Council guidance. Bmj 2008; 337:a1655.

37. Dorresteijn TA, Rixt Zijlstra GA, Van Haastregt JC, Vlaeyen JW, Kempen GI. Feasibility of a nurse-led in-home cognitive behavioral program to manage concerns about falls in frail older people: a process evaluation. Res Nurs Health 2013; 36(3): 257-70.

38. Bouwens SF, van Heugten CM, Verhey FR. Review of goal attainment scaling as a useful outcome measure in psychogeriatric patients with cognitive disorders. Dement Geriatr Cogn Disord 2008; 26(6): 528-40. 
Appendix 1. Partner in Balance - Coach Questionnaire

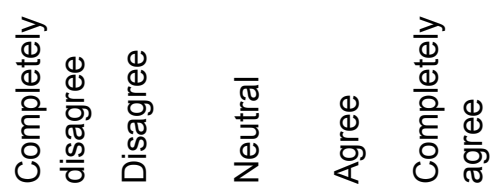

1. The program is feasible for coaches

2. I can combine the coach responsibilities with my usual activities

3. The program is a valuable contribution for caregivers

4. The program is a valuable contribution for coaches/professionals $\begin{array}{lllll}1 & 2 & 3 & 4 & 5\end{array}$

$\begin{array}{lllll}1 & 2 & 3 & 4 & 5\end{array}$

5. Was the program delivered according to protocol (in $+/-8$ weeks, feedback after each module, face-to-face intake and evaluation session). If not, what was performed inconsistent with the protocol and why?

6. How much time, on average, did you spent coaching one participant? (Based on the structured registration form)

7. What are the advantages of the program?

8. What are the disadvantages of the program? Points for improvement?

\section{Implementation}

9. What are the barriers for implementation of the program in your organization?

10. What could be possible facilitators for future implementation of the program within your organization? 



\section{CHAPTER 8}

Effectiveness of the blended care self-management program 'Partner in Balance' for early-stage dementia caregivers: results of a randomized controlled trial

Lizzy M.M. Boots, Marjolein E. de Vugt, Gertrudis I.J.M. Kempen \& Frans R.J. Verhey 


\section{ABSTRACT}

Background: The benefits of e-health support for dementia caregivers are increasingly recognized. Reaching early-stage dementia caregivers could prevent high levels of burden and psychological problems in the later stages. The current study evaluates the effectiveness of the blended care self-management program 'Partner in Balance' compared to a control group.

Methods/design: A mixed method single-blind, randomized controlled trial with 80 family caregivers of community-dwelling people with (very) mild dementia was conducted. Participants were randomly assigned to either the 8-week blended care self-management program 'Partner in Balance' or a waiting-list control group receiving usual care. Semi-structured interviews were conducted post-intervention. Primary proximal outcome included self-efficacy, primary distal outcome symptoms of depression. Secondary outcomes included mastery, psychological complaints (anxiety and perceived stress), and quality of life. Possible modifying effects of variables (quality of the relationship, neurotic personality, coach background) on the intervention effect were also evaluated.

Results: A significant increase in self-efficacy, mastery, and quality of life were demonstrated in the intervention group, compared with the control group. No significant differences were found between the groups on depressive symptoms, anxiety and perceived stress.

Discussion: The program could provide accessible care to future generations of early-stage dementia caregivers and strengthen the primary caregivers. 


\section{INTRODUCTION}

Family caregivers are important as the main source of care for people with dementia, ${ }^{1}$ but caregiving puts them at risk for feeling burdened, which may result in depression, anxiety and other health problems. ${ }^{2}$ Many caregiver support interventions have been developed to ameliorate negative caregiver consequences with promising results. ${ }^{3}$ Early intervention and support for caregivers could prevent high levels of burden and psychological problems in the later stages of dementia., However, early-stage interventions may not be effective, and even do more harm than good if they do not fit the personal situation of the caregiver. ${ }^{6}$ Negative and stigmatizing information can hamper acceptance, while enhancing the positive, intact experiences may be effective in increasing caregiver self-efficacy. By increasing caregiver resilience through self-efficacy, an increase of psychological problems in a later stage may be prevented. ${ }^{7}$ A self-management approach focused on learning to positively manage life with dementia could facilitate the adaptation process to the new caregiving role in the early stages. ${ }^{8}$

With the growing gap between the number of people in need of support and available care professionals ${ }^{9}$, e-health interventions could serve as cost-effective alternatives for dementia caregiver support ${ }^{10}$, with increased access and extended reach. $^{11-14}$ Blending face-to-face guidance with online support increases clienttherapist connection and adherence. ${ }^{15,16}$ E-health interventions for caregivers are being developed and evaluated, but are mainly aimed at dementia related problems in an advanced stage of the caregiver career ${ }^{17,18}$ and their overall quality of evidence is low. ${ }^{19}$ An iterative step-wise approach was employed to develop the blended care self-management internet-based program 'Partner in Balance' for early-stage dementia caregivers. The current study evaluated if 'Partner in Balance' is superior to a waiting-list control condition as evidenced by improved subjective self-confidence (self-efficacy and mastery), and lower levels of psychological complaints (symptoms of depression, anxiety and stress) post-intervention.

\section{METHODS}

This randomized controlled trial was carried out between 2014 and 2016 in the Netherlands. The 'Partner in Balance' program was compared to a waiting-list control group receiving usual care. The Medical Ethics Committee of the Maastricht University Medical Center+ (MUMC+) approved this study (\#12-4-059) and the study 
was registered in the Dutch trial register (NTR4748). The study protocol and supporting SPIRIT checklist are available. ${ }^{20}$

From September 2014 to December 2015, family caregivers of people with (very) mild dementia of all subtypes (Clinical Dementia Rating (CDR) score 0.5-1) [28] were recruited from memory clinics (MUMC+, Elkerliek Hospital Helmond, Catharina Hospital Eindhoven) and ambulatory mental health clinics (Virenze-RIAGG Maastricht, MET ggz Roermond) in the south of the Netherlands, via caregiver support services, and via the website of the Dutch Alzheimer Association (www.alzheimernederland.nl). Caregivers were included if they had access to the Internet at home, basic skills in the use of computers, and provided written informed consent. Potential participants with insufficient cognitive abilities to engage in the online self-management program; overburdened or with severe health problems as determined by study staff; or who cared for people with dementia caused by human immunodeficiency virus (HIV), acquired brain impairment, Down syndrome, chorea associated with Huntington's disease or alcohol abuse were excluded from participation. Both spouses and other caregivers could be included, e.g., children, as long as they met the criteria above and were $>18$ years. Details on the recruitment procedure are described in Chapter 7 .

\section{Randomization and masking}

Following the baseline assessment, participants were randomly assigned to either 'Partner in Balance' or the waiting-list control group receiving usual care, using a computerized random-number generator for block randomization with variable sizes of 4, 6 and 8 . An independent research assistant who was blinded to the allocation of the treatment conducted the post-intervention assessments.

\section{Intervention and control}

Experimental group: Partner in Balance (PiB). The 8-week blended intervention PiB consists of a face-to-face intake session with a personal coach, an online period, and a closing-out face-to-face evaluation session with the personal coach. The intervention is described in detail in Chapter $5 .{ }^{21}$ In short, in the face-to-face intake session with the personal coach participants were familiarized with the program, selected four out of nine available modules, and set goals that they wished to accomplish. Module themes were Acceptance, Balance in activities, Communication with family member and environment, Coping with stress, Focusing on the positive, 
Insecurities and rumination, Self-understanding, The changing family member, and Social relations and support. Following the intake, participants completed their chosen modules online during an 8-week period. Two weeks were allocated per module, but participants were allowed to complete the modules at their own pace in accordance with the self-management approach. ${ }^{22}$ After the online period, a face-toface evaluation session was conducted with the personal coach to discuss their ability to accomplish goals and to cope with future difficulties. Participants maintained access to their personal page and modules after the intervention period and were given the opportunity to follow additional modules without a time limit. The personal coaches were experienced psychologists $(\mathrm{N}=7)$ and psychiatric nurses $(\mathrm{N}=6)$ from memory clinics, ambulatory mental health clinics or caregiver support services in the south of the Netherlands. Coaches received a 2-hour training in self-management techniques, goal setting and online help, and regular supervision meetings where they could share experiences and ask feedback. Coaches supported participants in choosing modules, identifying feasible goals, and providing feedback on their assignments.

Control group: waiting-list. The waiting-list group received usual care consisting of non-frequent counseling during 8 weeks. They received the same pretest and post-test attention from the research team as the experimental group. After they completed the post-test assessment, they were given the opportunity to follow $\mathrm{PiB}$.

\section{Procedures}

For this study data from baseline visit $\left(T_{0}\right)$ and after 8 weeks $\left(T_{1}\right)$ were compared Data was collected with a mixed-methods approach; effectiveness was evaluated quantitatively (self-report) and qualitatively (semi-structured interview). The primary proximal outcome was caregiver self-efficacy and primary distal outcome was depressive symptoms. Caregiver self-efficacy was measured with The Caregiver Self-efficacy Scale (CSES), ${ }^{23}$ measuring care management self-efficacy (4 items) and service use self-efficacy ( 5 items). Care management self-efficacy scores range from 4-40 and service use self-efficacy from 5-50. The 20-item Centre for Epidemiological Studies Depression Scale (CES-D ${ }^{24}$ was used to measure depressive symptoms. Total scores range from 0-60. Secondary outcomes were mastery, psychological complaints (anxiety and perceived stress), and quality of life. Raw scores are transformed in individual mean GAS scores (T-scores). T-scores of 
$\geq 50$ indicate effective goal attainment. ${ }^{25}$ Mastery was measured with the 7-item Pearlin Mastery Scale (PMS). ${ }^{26}$ The total score ranges from 0-35. The 7-item Hospital and Anxiety Depression Scale anxiety subscale (HADS-A) ${ }^{27}$ rates symptoms of anxiety, with scores ranging from 0-21. Quality of life was measured on five attributes with the Investigating Choice Experiments for the Preferences of Older People CAPability measure for Older people (ICECAP-O) ${ }^{28}$ The value system for the 1024 states uses a best-worst scaling valuation method, providing a single summary score, anchored at zero ('no capability') and 1.0 ('full capability'). ${ }^{29}$

Demographics were obtained (sex, age, relationship to care recipient, level of education, sharing household, and care intensity in years). The Global Deterioration Scale (GDS) ${ }^{30}$ measured dementia severity with the caregiver as informant. Quality of the relationship was measured using 4 self-rating items of the University of Southern California Longitudinal Study of Three-Generation Families measures of positive affect. ${ }^{31}$ The 12-item Neuroticism domain of the NEO Five Factory Inventory $(N E O-F F I)^{32}$, was used to identifying individuals who are prone to psychological distress, by assessing 6 traits: anxiety, angry hostility, depression, selfconsciousness, impulsiveness and vulnerability. Scores ranged from 0-24.

Following the waiting-list period participants were offered the opportunity to follow the program. Qualitative data on the effect of PiB were obtained using a semistructured interview after completion of the intervention in both groups. The interviews were audiotaped with the participants' permission. Topics included the application of the intervention in daily life, and intervention impact on knowledge about the disease, caregiver functioning, and self-esteem.

\section{Data analysis}

Prior to the analysis, data was checked for missing values, outliers, and normality. Possible differences between the study groups' baseline characteristics were tested with $\mathrm{t}$-tests for continuous variables and $\mathrm{X}^{2}$-tests for categorical variables. Nonparametric tests were used when necessary in case of non-normality.

To examine differences between outcomes for the intervention and the waiting-list control group during the intervention period, an analysis of covariance (ANCOVA) was conducted with outcome at post intervention as the dependent variable, intervention (Partner in Balance, waiting-list control group) as the betweensubjects variable and per outcome its baseline value, age, sex, emotional instability, quality of the relationship, educational level and relationship to the care recipient as 
covariates. If significant, the inter-group effect size was calculated according to Cohen's $d$. Effect sizes of 0.2 are considered small, 0.5 is considered medium and 0.8 high. $^{33}$ IBM SPSS statistics 22.0 for Macintosh was used and all tests of significance reported mean change and were two-tailed with $\alpha$ set at 0.05 .

The qualitatively obtained data from the semi-structured interview was transcribed verbatim. Two independent researchers performed deductive content analysis of the transcribed text using ATLAS.ti (Version 1.0.14 for Macintosh). Conceptual labels were assigned to textual fragments and organized into categories. Categories were merged into common themes in a consensus meeting. Disagreements were solved through discussion.

\section{RESULTS}

\section{Participants}

A total of 163 caregivers expressed an interest to participate. See Figure 1 for the study flowchart. Details are described in Chapter 7. Table 1 lists the baseline data for the included caregivers $(\mathrm{N}=80)$.

Between-group comparisons revealed no significant differences in demographics and main outcome measures at baseline. Care recipients of the included caregivers were 73.9 years old (SD 8.2), diagnosed with $\mathrm{MCl}(12 ; 15 \%), A D$ (32; $40 \%)$, or other dementias (36; $45 \%)$. Dementia severity was rated as preclinical memory decline $(55 ; 68,8 \%)$, mild dementia $(23 ; 28.7 \%)$, or moderate dementia (2; $2,5 \%)$ on the GDS. At T1, 13 caregivers were lost to follow-up. The completers did not differ from non-completers at baseline in terms of age $(t=0.19, p=0.851)$, relationship to the care recipient $\left(X^{2}=1.39, p=0.238\right)$, same household as care recipient $\left(X^{2}=0.82, p=0.665\right)$, care intensity in years $(U=377.5, p=0.781)$, sex $\left(X^{2}=2.80, p=0.094\right)$, education $\left(X^{2}=1.20, p=0.550\right)$, self-efficacy service use $(t=0.53$, $p=0.599)$ care management $(t=1.36, p=0.177)$, depression $(U=280.0, p=0.266)$, stress $(t=0.25, p=0.806)$, anxiety $(U=372.0, p=0.497)$, mastery $(t=-1.18, p=0.253)$, and quality of life $(U=775.0, p=0.956)$. 


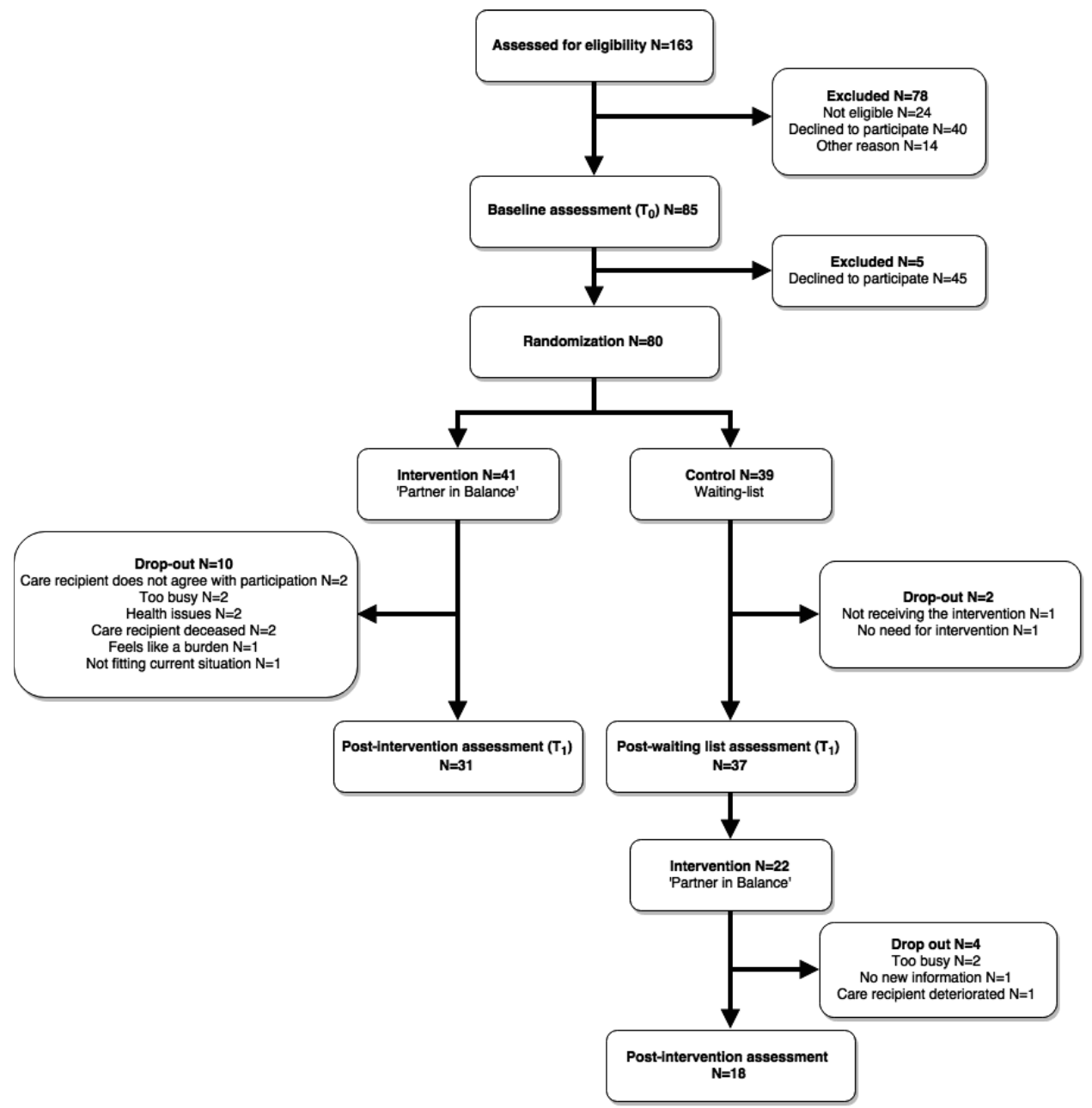

Figure 1. Study flowchart

\section{Intervention effects}

The effects were compared between groups (intervention and waiting-list control) after 8 weeks. Table 2 shows the results of the ANCOVA at T1 on self-efficacy (care management and service use), depression, mastery, perceived stress, anxiety, and quality of life. Examination of relationships between covariates and intervention outcomes showed a positive association between a higher quality of the relationship at baseline and improved self-efficacy care management $(B=1.18, p=0.019)$ and mastery $(B=0.72, p=0.007)$ post-intervention. Improvements on post-intervention quality of life were significantly related to lower scores on neurotic personality traits at baseline $(B=-0.01, p=0.037)$. Relationship to the care recipient, caregiver level of 
Table 1. Descriptive data caregivers for both groups at baseline

\begin{tabular}{|c|c|c|c|c|}
\hline & $\begin{array}{l}\text { Intervention } \\
(N=41)\end{array}$ & $\begin{array}{l}\text { Waiting-list } \\
(N=39)\end{array}$ & Test value & $P$ \\
\hline \multicolumn{5}{|l|}{ Socio-demographics } \\
\hline Age, mean (SD) & $67.8(10.2)$ & $70.2(10.1)$ & $T=1.0$ & 0.302 \\
\hline Spouse, mean (\%) & $37(90.2)$ & $37(94.9)$ & $x^{2}=0.6$ & 0.432 \\
\hline Same household as PwD, N (\%) & $39(95.1)$ & $37(94.9)$ & $x^{2}=1.3$ & 0.513 \\
\hline $\begin{array}{l}\text { Care intensity in years, mean } \\
(S D)\end{array}$ & $1.8(1.8)$ & $1.9(1.8)$ & $U=674.5$ & 0.929 \\
\hline Female, $N(\%)$ & $29(70.7)$ & $24(61.5)$ & $X^{2}=0.8$ & 0.385 \\
\hline \multicolumn{5}{|l|}{ Education $(N, \%)$} \\
\hline High school & $8(19.5)$ & $4(10.3)$ & $X^{2}=2.3$ & 0.321 \\
\hline College & $17(41.5)$ & $15(38.5)$ & & \\
\hline Graduate school & $15(36.6)$ & $20(51.3)$ & & \\
\hline \multicolumn{5}{|l|}{ Primary outcomes } \\
\hline \multicolumn{5}{|l|}{ Self-efficacy (CSES) } \\
\hline Care management & $34.7(7.8)$ & $33.0(9.4)$ & $T=-0.9$ & 0.395 \\
\hline Service use & $25.8(6.3)$ & $23.7(6.2)$ & $T=-1.5$ & 0.141 \\
\hline Depression (CES-D) & $13.1(8.7)$ & $13.1(9.0)$ & $U=732.0$ & 0.927 \\
\hline \multicolumn{5}{|l|}{ Secondary outcomes } \\
\hline Stress (PSS) & $11.8(6.0)$ & $13.5(6.2)$ & $\mathrm{T}=1.2$ & 0.223 \\
\hline Anxiety (HADS-A) & $6.0(3.7)$ & $6.7(4.7)$ & $U=717.5$ & 0.666 \\
\hline Mastery (PMS) & $23.7(4.1)$ & $22.9(4.4)$ & $T=-0.8$ & 0.430 \\
\hline Quality of life (ICECAP-O) & $0.8(0.1)$ & $0.8(0.1)$ & $U=755.0$ & 0.956 \\
\hline
\end{tabular}

education, and coach background were not significantly related to improvement on any of the outcomes. After controlling for age, sex, emotional instability, and quality of the relationship, significant effects in favor of the intervention group were found for self-efficacy care management $(F(1,60)=10.37, p=0.002 d=0.85)$, and self-efficacy service use $(F(1,60)=11.47, p=0.001, d=0.93)$, but not for depression $(F(1,60)=1.13$, $p=0.293)$. Significant effects in favor of the intervention group were also demonstrated for mastery $(F(1,60)=12.66, p=0.001, d=0.94)$, and quality of life $(F(1,60)=4.83, p=0.032, d=0.58)$, but not for perceived stress $(F(1,60)=3.40$, $p=0.071)$, and anxiety $(F(1,60)=0.80, p=0.374)$. 
Table 2. Analysis of covariance comparing intervention and control group at post-test

\begin{tabular}{|c|c|c|c|c|c|c|c|}
\hline \multirow{3}{*}{ Outcome } & \multicolumn{2}{|l|}{$\begin{array}{l}\text { Control } \\
(N=37)\end{array}$} & \multicolumn{5}{|c|}{$\begin{array}{l}\text { Intervention } \\
(N=31)\end{array}$} \\
\hline & \multicolumn{2}{|c|}{ Mean (SD/SE) } & \multicolumn{2}{|c|}{ Mean (SD/SE) } & \multirow{2}{*}{$\begin{array}{l}\text { Mean } \\
\text { difference }^{2} \\
(95 \% \mathrm{Cl})\end{array}$} & \multirow[t]{2}{*}{$F$} & \multirow[t]{2}{*}{$d$} \\
\hline & Crude $^{1}$ & Adjusted $^{2}$ & Crude $^{\top}$ & Adjusted $^{2}$ & & & \\
\hline \multicolumn{8}{|l|}{ Primary outcomes } \\
\hline \multicolumn{8}{|l|}{ CSES } \\
\hline Care management & $\begin{array}{l}31.38 \\
(8.71)\end{array}$ & $\begin{array}{l}31.65 \\
(1.05)\end{array}$ & $\begin{array}{l}37.03 \\
(6.33)\end{array}$ & $\begin{array}{l}36.73 \\
(1.12)\end{array}$ & $\begin{array}{l}-5.07 \\
(-8.23,-1.92)\end{array}$ & $10.37^{\times *}$ & 0.85 \\
\hline Service use & $\begin{array}{l}21.88 \\
(6.33)\end{array}$ & $\begin{array}{l}22.48 \\
(0.83)\end{array}$ & $\begin{array}{l}27.43 \\
(5.11)\end{array}$ & $\begin{array}{l}26.76 \\
(0.89)\end{array}$ & $\begin{array}{l}-4.274 \\
(-6.80,-1.75)\end{array}$ & $11.47^{* *}$ & 0.93 \\
\hline$C E S-D$ & $\begin{array}{l}13.27 \\
(9.21)\end{array}$ & $\begin{array}{l}12.87 \\
(1.08)\end{array}$ & $\begin{array}{l}10.73 \\
(8.20)\end{array}$ & $\begin{array}{l}11.17 \\
(1.14)\end{array}$ & $\begin{array}{l}1.70 \\
(-1.51,4.91)\end{array}$ & 1.13 & 0.28 \\
\hline $\begin{array}{l}\text { Secondary } \\
\text { outcomes }\end{array}$ & & & & & & & \\
\hline PMS & $\begin{array}{l}21.15 \\
(4.49)\end{array}$ & $\begin{array}{l}21.32 \\
(0.63)\end{array}$ & $\begin{array}{l}24.87 \\
(4.09)\end{array}$ & $\begin{array}{l}24.68 \\
(0.67)\end{array}$ & $\begin{array}{l}-3.36 \\
(-5.26,-1.47)\end{array}$ & $12.66^{* x}$ & 0.94 \\
\hline PSS & $\begin{array}{l}13.76 \\
(6.84)\end{array}$ & $\begin{array}{l}12.92 \\
(0.69)\end{array}$ & $\begin{array}{l}10.03 \\
(6.35)\end{array}$ & $\begin{array}{l}10.99 \\
(0.74)\end{array}$ & $\begin{array}{l}1.94 \\
(-0.17,4.04)\end{array}$ & 3.40 & 0.50 \\
\hline$H A D S-A$ & $\begin{array}{l}5.94 \\
(4.59)\end{array}$ & $\begin{array}{r}5.91 \\
(0.61)\end{array}$ & $\begin{array}{l}6.70 \\
(4.65)\end{array}$ & $\begin{array}{r}6.73 \\
(0.63)\end{array}$ & $\begin{array}{l}-0.81 \\
(-2.63,1.00)\end{array}$ & 0.80 & 0.24 \\
\hline ICECAP-O & $\begin{array}{l}0.76 \\
(0.15)\end{array}$ & $\begin{array}{l}0.76 \\
(0.02)\end{array}$ & $\begin{array}{l}0.82 \\
(0.10)\end{array}$ & $\begin{array}{l}0.83 \\
(0.02)\end{array}$ & $\begin{array}{l}-0.06 \quad(-0.12 \\
0.01)\end{array}$ & $-4.83^{*}$ & 0.58 \\
\hline
\end{tabular}

CSES: Caregiver Self-efficacy Scale; CES-D: Center for Epidemiological Studies Depression Scale; PMS: Pearlin Mastery Scale, PSS: Perceived Stress Scale, HADS-A: Hospital Anxiety and Depression Scale-Anxiety; ICECAP$O$ : Investigating Choice Experiments for the Preferences of Older People * $\mathrm{p}<0.05$ *** $p<0.01$ ${ }^{1}$ Group means

${ }^{2}$ Adjusted for outcome measure at baseline, age, sex, education, quality of the relationship at baseline, neurotic personality traits, and coach background

\section{Qualitative findings}

Semi-structured interviews $(\mathrm{N}=49)$ were held in both the intervention and waiting-list group post-intervention. Saturation occurred after coding of 40 semi-structured interviews. Coding and categorization resulted in 4 themes: Insight in behavior, increased acceptance and adjusted expectations, active coping, and positive emotions. Categories per theme are listed in Box 1. The themes are described below and illustrated with quotations to elucidate the results. 
Box 1. Overview of categories and major themes

\begin{tabular}{|ll|}
\hline THEMES & CATEGORIES \\
\hline Insight in behavior & Awareness of own behavior \\
Increased acceptance and & Confronting \\
adjusted expectations & Acceptance of new situation \\
& $\begin{array}{l}\text { Adjusting expectations } \\
\text { Increased understanding }\end{array}$ \\
Active coping & Taking control \\
& Openness in communication with environment \\
& Setting personal boundaries \\
& Armed with tools for the future \\
Positive emotions & Confirmation of propriety of own actions \\
& Mistakes are normal \\
& Supportive and reassuring \\
& Feel less lonely \\
Increased positive encounters with care recipient
\end{tabular}

\section{Insight in behavior}

Participants reported that the program made them more aware of their own behavior and its impact on the care recipient. Putting your emotions into words in the assignments increased their insight into their own feelings regarding the new situation. Some participants labeled the experience as confronting at first, but insightful when they reviewed their responses afterwards. Participants felt taken seriously because the difficulty of their task as caregivers was recognized.

\section{Active coping}

The awareness that many situations are within their own control gave participants hope for the future and the urge to think about what they would like to change. An active role decreased anxiety about possible future scenarios. Participants felt that they were challenged to explore their problems, uncover the causes, and actively seek solutions. This led to setting clear limits for themselves and protecting their own energy level.

"I feel that I did a lot myself, not just got offered advice. I'm kind of proud of that" [P72] 
"You're forced to analyze your problems, but you don't linger in them, you have to look for possible solutions. Don't reach for the stars, but try realistic things. That helped me to put my situation and feelings into perspective." [P28]

Increased acceptance and adjusted expectations

Some participants where frustrated that not every situation has a solution, while others experienced a more accepting attitude towards these situations. Knowledge that certain behaviors of the care recipient were disease-related and the realization that their own behavior accounted for a great deal of their perception of the situation, led participants to adjust their expectations about the future and live one day at a time. The acceptance of the situation as it is made participants more open about their situation to their social network, which decreased feelings of shame for the disease of their family member or for taking time for themselves.

\section{Positive emotions}

Recognition of their daily encounters and possible 'mistakes' one may experience in the examples in the program and the feedback of the coach decreased their feelings of loneliness and increased feelings of reassurance. Participants reported a shift from focus on loss to possibilities in day-to-day activities, which resulted in more positive encounters with the care recipient. As tension and worrying was seen as part of normal life, anxiety about the future was replaced with a feeling of being 'armed' for the future.

"Everything is about him (care recipient), so the fact that someone takes me seriously and tries to support me really helps, because you feel alone a lot of the time" [P03]

\section{DISCUSSION}

This controlled study evaluated the first blended care intervention for caregivers of people with early-stage dementia and demonstrated a significant improvement in care management self-efficacy, service use self-efficacy, mastery and quality of life after receiving the 'Partner in Balance' intervention, compared to a waiting-list control group receiving care as usual. Effect sizes were moderate $(>0.5)$ for quality of life to substantial $(>0.8)$ for self-efficacy and mastery. No differences between groups were demonstrated on caregiver depression, anxiety, and perceived stress. 
Results on caregiver self-efficacy, mastery, and quality of life are in line with previous results in an uncontrolled smaller sample ${ }^{21}$ and results of previous e-health interventions for dementia caregivers. ${ }^{19}$ Furthermore, the results fit the Stress and Coping paradigm by Lazarus and Folkman ${ }^{34}$ and the Social Learning theory by Bandura $^{7}$, suggesting that taking charge of the changes in one's life increases selfefficacy and general wellbeing. Learning to positively manage life with dementia instead of managing the dementia itself in a self-management program may have facilitated caregivers' adaptation to their new caregiving role. The program's focus on enhancing positive, intact experiences that are tailored to the individual caregiver's situation could explain the positive effects on caregiver self-efficacy. ${ }^{8}$ However, it was expected that a decrease in stress resulted in the demonstrated higher levels of wellbeing or quality of life ${ }^{7,34}$, which the present study could not demonstrate. It is conceivable that interventions aimed at the early stages may not be capable of decreasing burden and stress, as these are relatively low during the early stages ${ }^{6}$. Previous caregiver interventions demonstrating positive effects on burden and stress were not specifically aimed at early-stages of dementia. ${ }^{17,35-39}$ Future follow up of PiB effects could clarify if an increase in self-efficacy results in a decrease or prevention of increased stress and depression in the long term. Measures of self-efficacy are preferred over measures of sense of competence and distress for caregiver interventions in the early stages. ${ }^{40}$

\section{Strengths and limitations}

The mixed method evaluation approach enabled better understanding of the active intervention ingredients and qualitative data helped clarify the quantitative results.

High face validity was demonstrated as the program was evaluated in multiple institutions with multiple coaches of different backgrounds. Development together with the potential users and a pilot evaluation following the MRC Framework may have increased its effectiveness.

The waiting-list period may have affected the differences in outcomes between both groups. However, this design allowed all potentially interested participants to participate in the intervention program, which may have increased their motivation to participate given that usual care for (very) mild dementia caregivers often either does not include counseling or includes only very infrequent counseling. ${ }^{41}$ Furthermore, the waiting-list group was not deprived of usual care. An alternative would be a pseudo-intervention in which only psycho-education or only attention of the coach is 
provided, but the aim of this study was not to evaluate the online aspect of the intervention only, but the effect of the blended-care intervention of which psychoeducation and face-to-face contacts are integral parts.

Intention-to-treat analyses was not fully possible, as intervention noncompleters refused to participate in further assessments. However, we did include participants that were not completely compliant (completed only 2, 3 or no modules at all) in the analyses. ${ }^{42}$ Drop-out was higher in the intervention group compared to the control group, but selective drop-out was not demonstrated as completers did not differ from non-completers. Often mentioned reasons for drop-out were no need for help or refusal by the care recipient, which was demonstrated previously as reasons of non-use of formal services. ${ }^{43,44}$ Furthermore, a higher rate of drop-out in the intervention group has previously been reported and previous RCT's even controlled for this phenomenon beforehand by increasing the sample of the intervention group. Although the power of our group was not jeopardized based on our previous power calculation, future studies could consider controlling for a higher rate of drop-out in the intervention group to prevent loss of power.

Our sample was not limited to memory clinics only, but the included participants may represent a subgroup of all dementia caregivers in the early stages. Caregivers in the early stages often decline formal care and it is conceivable that many were not familiar with the care parties involved in recruitment and were therefore overlooked in this study. ${ }^{43,44}$ This could have resulted in a highly motivated sample more open to support. ${ }^{40}$ Furthermore, only computer-literature caregivers could be included, which represents only $59.1 \%$ of dementia caregivers. ${ }^{45}$ However, seniors' use of Internet is expected to rise in the near future ${ }^{46}$, increasing the accessibility of $\mathrm{PiB}$.

\section{Future research and clinical implications}

Future research could consider combing all resources used during the intervention period with the intervention costs and outcomes in a cost-consequence analysis to aid decision makers. Furthermore, sustainability of improvements will be evaluated at long-term follow-up. 


\section{Conclusion}

In conclusion, this study showed that a blended care self-management program for dementia caregivers in the early stages is effective in increasing caregiver selfefficacy, mastery and quality of life. The program could provide accessible care to future generations of early-stage dementia caregivers and strengthen the primary caregivers. 


\section{References}

1. Association As. Alzheimer's Disease Facts and Figures. Alzheimers Dement 2014; 10(2).

2. Brodaty $\mathrm{H}$, Donkin M. Family caregivers of people with dementia. Dialogues Clin Neurosci 2009; 11(2): 217-28.

3. Olazaran J, Reisberg B, Clare L, et al. Nonpharmacological therapies in Alzheimer's disease: a systematic review of efficacy. Dement Geriatr Cogn Disord 2010; 30(2): 161-78.

4. Gaugler JE, Kane RL, Kane RA, Newcomer R. Early community-based service utilization and its effects on institutionalization in dementia caregiving. Gerontologist 2005; 45(2): 177-85.

5. Kjallman Alm A, Hellzen O, Norbergh KG. Experiences of long term ongoing structured support in early stage of dementia - a case study. Int $\mathrm{J}$ Older People Nurs 2013.

6. de Vugt ME, Verhey FR. The impact of early dementia diagnosis and intervention on informal caregivers. Prog Neurobiol 2013; 110: 54-62.

7. A. B. Self-Efficacy: The Exercise of Control. . New York: W.H. Freeman and Company; 1997.

8. Boots LM, Wolfs CA, Verhey FR, Kempen GI, de Vugt ME. Qualitative study on needs and wishes of early-stage dementia caregivers: the paradox between needing and accepting help. Int Psychogeriatr 2015; 27(6): 927-36.

9. Macdonald A, Cooper B. Long-term care and dementia services: an impending crisis. Age Ageing 2007; 36(1): 16-22.

10. Lewis BA, Williams DM, Neighbors CJ, Jakicic JM, Marcus BH. Cost Analysis of Internet vs. Print Interventions for Physical Activity Promotion. Psychol Sport Exerc 2010; 11(3): 246-9.

11. Davis Kirsch SE, Lewis FM. Using the World Wide Web in Health-related intervention research. CIN: Computers, Informatics, Nursing 2004; 22(1): 8-18.

12. Harrell KM, Wilkins SS, Connor MK, Chodosh J. Telemedicine and the evaluation of cognitive impairment: the additive value of neuropsychological assessment. J Am Med Dir Assoc 2014; 15(8): 600-6.

13. Serafini JD, Damianakis T, Marziali E. Clinical practice standards and ethical issues applied to a virtual group intervention for spousal caregivers of people with Alzheimer's. Soc Work Health Care 2007; 44(3): 225-43. 
14. Robinson L, Brittain K, Lindsay S, Jackson D, Olivier P. Keeping In Touch Everyday (KITE) project: developing assistive technologies with people with dementia and their carers to promote independence. Int Psychogeriatr 2009; 21(3): 494-502.

15. van der Vaart $R$, Witting M, Riper H, Kooistra L, Bohlmeijer ET, van GemertPijnen LJEWC. Blending online therapy into regular face-to-face therapy for depression: content, ratio and preconditions according to patients and therapists using a Delphi study. BMC Psychiatry 2014; 14(355).

16. Wilhelmsen M, Lillevoll K, Risør MB, et al. Motivation to persist with internetbased cognitive behavioural treatment using blended care: a qualitative study. BMC Psychiatry 2013; 13(296).

17. Hayden LJ, Glynn SM, Hahn TJ, Randall F, Randolph E. The Use of Internet Technology for Psychoeducation and Support With Dementia Caregivers. Psychol Serv 2012; 9(2): 215-8.

18. Blom M, Bosmans JE, Cuijpers P, Zarit SH, Pot AM. Effectiveness and costeffectiveness of an Internet intervention for family caregivers of people with dementia: design of a randomized controlled trial. BMC Psychiatry 2013; 13(17).

19. Boots LM, de Vugt ME, van Knippenberg RJ, Kempen GI, Verhey FR. A systematic review of Internet-based supportive interventions for caregivers of patients with dementia. Int J Geriatr Psychiatry 2014; 29(4): 331-44.

20. Boots LMM, de Vugt ME, Kempen GIJM, Verhey FRJ. Effectiveness of the blended care self-management program 'Partner in Balance' for early-stage dementia caregivers: study protocol for a mixed-methods single-blind randomized waiting-list controlled trial. Trials (submitted) 2016.

21. Boots L, De vugt M, Withagen H, Kempen G, Verhey Fip. Development and initial evaluation of the web-based self-management intervention 'Partner in Balance' for family caregivers of people with early-stage dementia: an exploratory mixed-methods study. JMIR research protocols 2016.

22. Lorig KR, Holman $H$. Self-management education: history, definition, outcomes, and mechanisms. Ann Behav Med 2003; 26(1): 1-7.

23. Fortinsky $\mathrm{RH}$, Kercher $\mathrm{K}$, Burant $\mathrm{CJ}$. Measurement and correlates of family caregiver self-efficacy for managing dementia. Aging Ment Health 2002; 6(2): $153-60$. 
24. Radloff LS. The CES-D Scale: A Self-Report Depression Scale for Research in the General Population. Applied Psychological Measurement 1977; 1: 385401.

25. Kiresuk TJ, Smith A, Cardillo JE. Goal attainment scaling: Applications, theory, and measurement. Hillsdale, NJ, England: Lawrence Erlbaum Associates, Inc.; 1994.

26. Pearlin LI, Schooler C. The structure of coping. J Health Soc Behav 1978; 19(1): 2-21.

27. Zigmond AS, Snaith RP. The hospital anxiety and depression scale. Acta Psychiatr Scand 1983; 67(6): 361-70.

28. Grewal I, Lewis J, Flynn T, Brown J, Bond J, Coast J. Developing attributes for a generic quality of life measure for older people: preferences or capabilities? Soc Sci Med 2006; 62(8): 1891-901.

29. Coast J, Flynn TN, Natarajan L, et al. Valuing the ICECAP capability index for older people. Soc Sci Med 2008; 67(5): 874-82.

30. Reisberg B, Ferris SH, de Leon MJ, Crook T. Global Deterioration Scale (GDS). Psychopharmacol Bull 1988; 24(4): 661-3.

31. Lawrence RH, Tennstedt SL, Assmann SF. Quality of the caregiver--care recipient relationship: does it offset negative consequences of caregiving for family caregivers? Psychol Aging 1998; 13(1): 150-8.

32. Costa PT, McCrae RR. NEO PI-R: Professional manual. Odessa, FL: Psychological Assessment Resources; 1992.

33. Cohen J. Statistical power analysis for the behavioral sciences. Hillsdale, NJ: Lawrence Earlbaum Associates; 1988.

34. Lazarus R, Folkman S. Stress, appraisal and coping. New York: Springer; 1984.

35. Hattink B, Meiland F, van der Roest $H$, et al. Web-Based STAR E-Learning Course Increases Empathy and Understanding in Dementia Caregivers: Results from a Randomized Controlled Trial in the Netherlands and the United Kingdom. J Med Internet Res 2015; 17(10): e241.

36. Blom MM, Zarit SH, Groot Zwaaftink RB, Cuijpers P, Pot AM. Effectiveness of an Internet intervention for family caregivers of people with dementia: results of a randomized controlled trial. PLoS One 2015; 10(2): e0116622.

37. Kajiyama B, Thompson LW, Eto-Iwase T, et al. Exploring the effectiveness of an internet-based program for reducing caregiver distress using the iCare 
Stress Management e-Training Program. Aging Ment Health 2013; 17(5): 54454.

38. Cristancho-Lacroix V, Wrobel J, Cantegreil-Kallen I, Dub T, Rouquette A, Rigaud AS. A web-based psychoeducational program for informal caregivers of patients with Alzheimer's disease: a pilot randomized controlled trial. J Med Internet Res 2015; 17(5): e117.

39. van der Roest HG, Meiland FJM, Jonker C, Droes RM. User evaluation of the DEMentia-specific Digital Interactive Social Chart (DEM-DISC). A pilot study among informal carers on its impact, user friendliness and, usefulness. Aging Ment Health 2010; 14(4): 461-70.

40. Banningh LW, Vernooij-Dassen MJ, Vullings M, Prins JB, Rikkert MG, Kessels RP. Learning to live with a loved one with mild cognitive impairment: effectiveness of a waiting list controlled trial of a group intervention on significant others' sense of competence and well-being. Am J Alzheimers Dis Other Demen 2013; 28(3): 228-38.

41. Campbell M, Fitzpatrick $R$, Haines $A$, et al. Framework for design and evaluation of complex interventions to improve health. Bmj 2000; 321(7262): 694-6.

42. Boots LMM, de Vugt ME, Smeets CMJ, Kempen GIJM, Verhey FRJ. Validity and implementation of the blended care self-management program 'Partner in Balance' for caregivers of people with early-stage dementia: a process evaluation. In preparation 2016.

43. Brodaty $\mathrm{H}$, Thomson $\mathrm{C}$, Thompson $\mathrm{C}$, Fine M. Why caregivers of people with dementia and memory loss don't use services. Int J Geriatr Psychiatry 2005; 20(6): 537-46.

44. Wolfs $C A$, de Vugt ME, Verkaaik M, et al. Rational decision-making about treatment and care in dementia: A contradiction in terms? Patient Educ Couns 2011.

45. Kim H. Understanding Internet Use Among Dementia Caregivers: Results of Secondary Data Analysis Using the US Caregiver Survey Data. Interactive journal of medical research 2015; 4(1): e1.

46. Eastman JK, lyer R. The elderly's uses and attitudes towards the Internet. $J$ Consum Marketing 2004; 21: 208-20. 



\section{CHAPTER 9}

General Discussion 


\section{INTRODUCTION}

People with Mild Cognitive Impairment or early-stage dementia are often left with a diagnosis but no support. Research indicates that although interference in daily life is still low, people with cognitive problems and their family members do struggle with the changes that occur and the uncertainty about their future. ${ }^{1,2}$ Efficacious pharmacotherapies are not yet available to cure cognitive disorders, but an early diagnosis offers opportunities to improve early adaptation to the caregiver role. In turn, this may prevent high levels of burden and institutionalization of the person with the dementia in the later stages. ${ }^{3,4}$ However, the evidence for early-stage caregiver support was not yet conclusive. ${ }^{5}$ E-health self-management interventions could suit the caring role transition caregivers face during the early stages. ${ }^{6}$ The main aim of this thesis was to develop and evaluate an e-health self-management intervention for caregivers of people with early-stage dementia. In this thesis we explored the state of the art with respect to the evidence of e-health interventions for caregivers of people with dementia (Chapter 2), the problems of caregivers of people with early-stage dementia and their needs for care and support (Chapter 3 and 4), the content and feasibility of the newly developed program 'Partner in Balance' (Chapter 5) and its process and effects on self-efficacy, mastery, and psychological complaints (Chapter 7 and 8$)$.

This chapter starts with an overview of the main findings of this thesis and discusses their scientific interpretation and implications. Next, some methodological considerations are discussed, together with the implications for clinical practice and future research.

\section{Main findings and implications}

\section{E-health interventions for caregivers of people with dementia}

Internet interventions to support dementia caregivers are relatively new but show promising results on caregiver confidence, self-efficacy, stress, burden, and depression (Chapter 2). Although quality of the evidence is still low, several successful elements could be identified, such as combining tailored information with the help of a coach and offering possibilities to interaction with other caregivers. These results are in line with the conclusions of previous studies emphasizing the importance of guidance by a coach and caregiver interaction. ${ }^{7,8}$ Furthermore, 
previous reviews have shown that caregiver interventions are more effective if they are tailor made and fit the needs of the caregiver., 9,10

\section{Problems and needs of caregivers of people with early-stage dementia}

A further qualitative exploration of these needs showed that there is a mismatch between supply and demand of informal support. Barriers to supply support were partly similar to those identified in earlier research, such as a fear of stigma, ${ }^{11}$ negative attitudes towards accepting support, for example a sense of responsibility, and unwillingness to relinquish support to others ${ }^{12}$. The identified mismatch was strengthened by an observed cognitive bias. Both the spousal caregivers and the social network members tended to think for others and exhibited unverified and sometimes incorrect thoughts concerning each other's intentions, resulting in not explicitly communicating support wishes and opportunities. According to Beck's cognitive theory, people can become susceptible to interpreting the intentions of others in a distorted or biased way because meanings are derived from personal experiences and the way people structure the world ${ }^{13,14}$. Open communication in formal and informal care systems might repair this mismatch (Chapter 3 ). This finding underscores the importance of early education and social network interventions to facilitate positive interactions. Early caregiver interventions ${ }^{15} 16,17$ might effectively improve support and open communication to prevent these cognitive biases. However, it was not clear whether and which early-stage interventions are desired. To tailor interventions accordingly and maximize the benefit of an early-stage intervention, the problems and needs for care of caregivers of people with earlystage dementia were explored (Chapter 4). Results showed that early-stage caregivers found it difficult to acknowledge care needs in an early phase of the disease due to fear of stigma and low acceptance. Fear is linked with an urge to escape ${ }^{18}$, which could explain why caregivers often postpone services utilization until care becomes an acute necessity. ${ }^{19}$ Previous research showed that next to stigma, ${ }^{20}$ a lack of awareness, no perceived need of such services, or fear of care recipients' reactions ${ }^{21}$ might explain non-use of services. Better understanding of the disease, increasing personal time, structuring ones day and using appropriate humor can reduce negative communication. In turn, this may increase positive encounters and caregiver-confidence, contributing to positive interaction with the care recipient and an increase in well-being. This focus on adaptation and positive aspects of caregiving fits the broaden and build theory of positive emotions, stating that certain positive 
emotions, such as joy, interest, contentment, pride, and love all share the ability to broaden people's momentary thought-action possibilities. ${ }^{22}$ This broadened mindset has indirect and long-term adaptive benefits, as it builds enduring personal resources, which can be used to manage future threats. For instance, Chapter 3 discussed that caregivers do not want to ask their social network members for help, but do want to strengthen positive experiences and emotions within the social network, which can result in a lowered threshold to ask for help when it is inevitable. Consistent with this finding, caregiver intervention research might focus on strengthening aspects of health. As ageing with chronic illnesses has become more 'normal' than divergent, the current WHO definition of health is counterproductive, declaring that people with chronic diseases and disabilities are by definition ill. A newly developed concept of health emphasizes people's capacity to fulfill their potential and obligations, manage their life with some degree of independence and engage in social activities, despite their medical condition or that of their loved one ${ }^{23}$. From this perspective, health is a dynamic balance between opportunities and limitations. If people are able to successfully cope with a difficult situation by enhancing its comprehensibility, manageability and meaningfulness, the perceived quality of life does not have to deteriorate. ${ }^{24}$ Our data confirmed that participation in social activities are highly valued and that a positive focus on opportunities, rather than limitations, might facilitate positive social interactions and supportive behavior. Furthermore, the meaningfulness of caregiving can be found in the positive aspects of caregiving. Next to the negative consequences of caregiving, caring can also be rewarding and provide a sense of self-efficacy and feelings of accomplishment. ${ }^{25}$ Support interventions may include cognitive behavioral techniques that address maladaptive thoughts regarding caregiver support. For example, cognitive restructuring and positive reappraisal techniques may shed a more positive light on seeking informal support, such as the realization that providing or asking for support does not only impose a burden or stigma but, in contrast, might lead to a reciprocal enrichment of relationships and increased social functioning.

\section{Content and feasibility of an e-health self-management program}

Fitting the new concept of Health emphasizing people's capacity and use of resources, the Stress and Coping paradigm by Lazarus and Folkman ${ }^{26}$ and the Social Learning theory by Bandura ${ }^{27}$ propose that taking charge of the changes in one's life promotes healthier behavior, increases self-efficacy, and can therefore 
reduce caregiver stress and its impact on general wellbeing. ${ }^{28}$ Following these theories and the findings discussed above, a support program for caregivers in the early stages of their caregiving role could be aimed at positively managing life with dementia rather than managing the dementia itself. ${ }^{29}$ Self-management programs suit the caring role transition and have previously been used to support informal caregivers of several chronic diseases with promising results. ${ }^{30-32}$

To this end, we developed the blended care self-management program 'Partner in Balance' and evaluated its feasibility and preliminary effects (Chapter 5). This tailored intervention approach appeared to be feasible for informal EDC and supported them in the difficulties of caregiving. The convenience of completing the program at home, the tailored content, and the guidance (face-to-face and online) were appraised positively. Previous self-management studies confirm that personal caregiver needs should be used as a starting point. ${ }^{33}$ As guidance from a 'real life' person was often desired, a blended care format was chosen. The value of this format has been supported by previous studies; participants have felt connected to the coach or therapist delivering the intervention ${ }^{34}$ and have been more motivated to persist with web-based interventions. ${ }^{35}$ Preliminary effects on caregiver self-efficacy were promising. This finding is in line with Bandura's theory; when equipped with the tools needed to make a change towards a more desired situation, caregivers' objective understanding of the situation and their beliefs about their ability to manage the situation, e.g., their self-efficacy level, may increase. ${ }^{27}$ Furthermore, the finding is congruent with previous research on online support for dementia caregivers. ${ }^{7,36,37}$ Confirming the feasibility and preliminary effectiveness is a valuable step towards examining the effectiveness of a newly developed intervention. ${ }^{38,39}$

\section{Process and effects of 'Partner in Balance'}

Feedback from the feasibility study was used to adapt the program to increase userfriendliness. Next, we aimed to evaluate the process characteristics and effectiveness of 'Partner in Balance'. The process evaluation (Chapter 7 ) showed that the participation rate of recruited caregivers was $52 \%$. Considering an average response rate of $27 \%$ for caregiver research, our participation rate can be considered substantial. ${ }^{40}$ However, the $52 \%$ only considered eligible caregivers who where already familiar with the care parties involved in recruitment and may have been more motivated and open to support ${ }^{41}$. Recruitment barriers reported by coaches were e.g. no computer, no need for intervention or additional support, which were 
also reasons to decline participation. As mentioned in Chapter 4, caregivers found it difficult to acknowledge care needs in an early phase of the disease due to fear of stigma, shame and low acceptance, which could explain this finding. Young age and being employed were considered recruitment facilitators. However, caregivers of people with young-onset dementia and children had difficulties identifying with examples about older caregivers. Previous research confirmed that younger caregivers struggle with different aspects in daily life, when compared to older caregivers ${ }^{42}$. It is essential that to match program content and specific needs of the target audience to maximize the benefit of a supportive intervention ${ }^{9,16,43,44}$. The thematic structure of the program allows for add-on of specific themes for subgroups, such as caregivers of people with young-onset dementia.

Both participants and coaches mentioned that their relationship with each other had deepened, which was also demonstrated in a previous blended-care intervention for depression ${ }^{34}$. Previous research confirmed that the opportunity to reflect on one's feelings anonymously in one's personal safe environment is easier than face-to-face, but the face-to-face contact increased caregiver openness, and therefore coach empathy with their situation ${ }^{35}$. Participant compliance to all 4 modules was high $(87.8 \%)$, which could be explained by the motivational aspect of having a coach, also mentioned in previous studies ${ }^{35}$. The varying intervention period and dose may influence the effectiveness of the intervention ${ }^{45}$. However, reasons for protocol deviations were diverse and not uncommon for informal caregivers and elderly participants (e.g. caregiver pace, time constraints, illness) ${ }^{40}$. In self-management interventions the participant is in control and should therefore be allowed to complete the modules at his own pace ${ }^{46,47}$. Furthermore, complex interventions such as Partner in Balance are often designed to be implemented with some flexibility, in order to accommodate differences across participants ${ }^{48}$.

The low level of professional involvement in the self-management approach was considered a challenge. The performance of the coach in self-management care is very important for the participant's performance of self-management tasks and overall intervention effectiveness ${ }^{49}$. It was previously argued that health care providers do not always support self-management education, as this is not part of their attitudes, beliefs or regular care practice ${ }^{47}$. Therefore, it can be recommended to increase the essential clinical competences of healthcare providers to provide tailored self-management support ${ }^{44}$, and their awareness of the benefits of evidencebased self-management programs for their target population ${ }^{47}$. Partner in Balance 
was evaluated within multiple organizations with coaches from different backgrounds, who received training and supervision in self-management and blended care. A relatively high response rate of organizations to adopt the program was found $(73 \%)$, which could be attributed to the current demand of health care insurances for care organizations to provide e-health ${ }^{50}$. However, a lack of financing and time could hamper the implementation. It is important to consider the context and level of implementation during the first phases of intervention development and evaluation, as many psychosocial interventions show different results in different settings or countries ${ }^{38,51}$

A randomized controlled trial was executed to demonstrate the effects of Partner in Balance (Chapter 8). The controlled study demonstrated a significant increase in self-efficacy, mastery, and quality of life in the intervention group (Partner in Balance), compared to a waiting-list control group receiving usual care. No significant differences were found between the groups on depressive symptoms, general distress and perceived stress. These results are in line with previous results in the pilot study (Chapter 7) and other e-health interventions for dementia caregivers. ${ }^{16}$ The program focused on enhancing positive, intact experiences that are tailored to the individual caregiver's situation, with a positive outcome on self-efficacy and quality of life. This suggests that positive emotions should not just be considered as a result of support, but could also be adopted as a mean to achieve psychological growth and improved well-being over time. For instance, the distinct positive emotion that follows personal achievements, pride, broadens the mind by creating an urge to share achievements with others and envision greater achievements in the future. In Chapter 8, participants reported more openness with their environment following the intervention, which could be attributed to the positive emotion pride experienced by attaining their goals. ${ }^{52}$ However, it was expected that a decrease in stress caused the demonstrated higher levels of wellbeing or quality of life ${ }^{26,27}$, which the effect study could not demonstrate. It is conceivable that interventions aimed at the early stages may not be capable of decreasing burden and stress, as these are relatively low during the early stages. ${ }^{6}$ Previous caregiver interventions demonstrating positive effects on burden and stress were not specifically aimed at early-stages of dementia. ${ }^{53-58}$ Follow-up of effects could clarify if an increase in self-efficacy postintervention is maintained in the long term and results in a decrease or prevention of increased stress and depression. 


\section{Methodological considerations}

Each chapter in this thesis has its methodological strengths and weaknesses, which we discuss below.

\section{Strengths}

In this thesis we made use of an iterative process recommended by the new Medical Research Council (MRC) framework ${ }^{38}$ by exploring the existing evidence, theoretical frameworks and user- and professional input, which led to the development of a program based on scientific knowledge, clinical experience and needs of the target audience. The mixed method methodology facilitated replication through greater knowledge of the active component(s) and potential barriers to implementation. ${ }^{59}$ The evaluation of the process and effects of Partner in Balance in different contexts increased the generalizability of the results. ${ }^{60}$ These type of pragmatic trials are recommended by the MRC Framework, as they may prevent different intervention outcomes in different settings and countries. ${ }^{38}$

\section{Limitations}

First, the systematic review used two different quality appraisals to rate and categorize the investigated studies, which may have led to exceedingly negative evaluations. Also, the methodological quality was rated with a set of criteria based on the Consolidated Standards of Reporting Trials (CONSORT) criteria, which can be difficult for psychosocial research to comply to. ${ }^{61}$

Second, the waiting-list period may have affected the differences in outcomes between both groups. The effects of waiting are highly variable and depend on the characteristics of the sample and of the trial. ${ }^{62}$ However, this design allowed all potentially interested participants to participate in the intervention program, which may have increased their motivation to participate given that usual care for (very) mild dementia caregivers often either does not include counseling or includes only very infrequent counseling. ${ }^{63}$ Furthermore, the waiting-list group was not deprived of usual care. An alternative would be a pseudo-intervention in which only psychoeducation or only attention of the coach is provided, but the aim of this study was not to evaluate merely the online aspect of the intervention, but the effect of the blendedcare intervention of which psycho-education and face-to-face contacts are integral parts. 
Third, intention-to-treat analysis was not fully possible, as intervention noncompleters refused to participate in further assessments. However, we did include participants that were not completely compliant (completed only 2, 3 or no modules at all) in the analyses. ${ }^{64}$ Drop-out was higher in the intervention group compared to the control group, but selective drop-out was not demonstrated as completers did not differ from non-completers. Often mentioned reasons for drop-out were no need for help or refusal by the care recipient, which was demonstrated previously as reasons of non-use of formal services. ${ }^{21,41}$

Finally, our participants may represent a subgroup of all dementia caregivers in the early stages. Caregivers in the early stages often decline formal care and it is conceivable that many were not familiar with the care parties involved in recruitment and were therefore overlooked in this study. ${ }^{21,41}$ This could have resulted in a highly motivated sample more open to support. ${ }^{5}$ The studies in this thesis also encompassed a high percentage of spousal caregivers compared to other family caregivers. Previous research indicated that especially people with dementia with spousal caregivers could benefit from early diagnosis and intervention ${ }^{65}$, which the present study was not able to demonstrate, as the power for subgroup analyses was not sufficient. Furthermore, only computer-literature caregivers could be included, which represents only $59.1 \%$ of dementia caregivers. ${ }^{66}$ However, seniors' use of Internet is expected to rise in the near future ${ }^{67}$, increasing the accessibility of e-health programs such as Partner in Balance. Younger generations are familiar with new technology and more and more elderly seem to get acquainted with a computer or tablet. $^{67}$

\section{Clinical implications}

A dementia diagnosis might be stigmatizing and put people with dementia and their caregivers outside of society. ${ }^{20}$ The research conducted in this thesis aimed to support the caregivers of people that received the widely advocated early diagnosis of dementia.

First, this thesis showed that reaching and effectively supporting caregivers in the early stages is inconsistent with the negative view on dementia as a horrifying disease with merely adverse consequences. The current focus on dementia as a terrible disease might lead to fear of stigma in caregivers and should therefore be adapted to a more positive view in which people with dementia and their caregivers can focus on their capacity to fulfill their potential and obligations, manage their life 
with some degree of independence and engage in social activities, despite the diagnosis. $^{23}$

Second, the thesis showed that an active support approach aimed at selfmanagement like the Partner in Balance program successfully supports caregivers in the early stages of dementia. The program could therefore be a valuable support option for dementia caregivers in the early stages. Involving caregivers in their choice of support is important, as previous research showed that actively involving caregivers in treatment decision-making sets aside effective from ineffective support programs, ${ }^{68}$ and offering choices may result in a greater sense of personal control, better connection with personal needs, more satisfaction with treatment, higher adherence and consequently better outcomes. ${ }^{6}$ However, it can be argued that earlystage support should not be offered when people do not express a specific need for support, to avoid stigmatization. Stigma in dementia is still persistent and can be defined as a process of exclusion whereby a 'normal' person is reduced to a person with whom something is wrong. ${ }^{6}$ Moreover, as Zarit and Femia ${ }^{69}$ put it: "A trial that enrolls caregivers who don't need help in a specific area provides treatment for a problem that doesn't exist". However, even in an early stage caregivers experience distress and depressive symptoms, which may be related to changes in family roles and relationships ${ }^{2,70}$ and common neuropsychiatric symptoms like apathy that undermine relationship reciprocity. ${ }^{1,6}$ Furthermore, the early-stage needs paradox described in Chapter 3 showed that caregivers in later stages desired receiving support earlier-on, but caregivers in the early stages struggled with acknowledging care needs. This was also demonstrated in Chapter 7 where reasons for declining participation and inability to set goals were, amongst others, not needing support or an intervention. It was argued that this paradox could be attributed to low levels of acceptance in the early stages. However, it should not be disregarded that this program is not suitable for all caregivers. The higher rate of drop-out in the intervention group showed that this program can be considered as burdensome and e-health interventions in general are not appropriate for caregivers that are not computer-literate or have more practical care needs. Nonetheless, a relatively high response and participation rate was found, indicating that there is a need for at least having the option to choose for this type of caregiver support. An active role of health care professionals in outlining care and support options early in the dementia process is recommended to increase the possibility that caregivers receive the support that fits their needs, which can consequently help caregivers involve the 
person with dementia in making medical, financial, legal and care decisions. ${ }^{71}$

Finally, the effectiveness of the program may be particularly attributed to the blended aspect, as was argued in Chapter 7. Previous research also suggests that blended care clients feel connected to the coach or therapist delivering the intervention and are more motivated to persist with Internet-based interventions. ${ }^{35}$ Furthermore, blending online modules with face-to-face care offers the opportunity to enhance self-management and cost-effectiveness of an intervention, while stile providing the support caregivers need. ${ }^{34}$ It is recommended that Partner in Balance is adopted in its current blended care format to optimize its effectiveness. If clinicians choose to implement Partner in Balance, it can be recommended to use an instrument like Goal Attainment Scaling to assess caregiver needs, as goal attainment may have a positive effect on feelings of control and self-efficacy. Selfmanagement studies also stress that personal caregiver needs should be taken as a starting point of support. ${ }^{33}$

We can conclude that people need to be informed about support possibilities following diagnosis, their need for support should be assessed and shared decision making should be adopted when it comes to decisions about caregiver support. Positive aspects of caregiving should be promoted to protect caregivers against negative health outcomes.

\section{Future research directions}

Our thesis showed that psychosocial research for dementia caregiver support lacked methodological rigor. First, it can be recommended that more high quality studies (randomized controlled trials; RCTs) are needed to make stronger statements about the effects of supportive Internet interventions and their most promising elements. However, while an RCT is the golden standard for determining the effectiveness of an intervention, the use of an RCT design in complex psychosocial interventions can be difficult because of their often unblinded and multi-component character. ${ }^{38,61}$ Furthermore, an RCT is not the only rigorous method for evaluating interventions; many alternatives exist when RCTs are not possible or appropriate due to scientific, practical, and ethical concerns, such as observational, quasi-experimental, and qualitative studies. Also, pragmatic trials (trials designed to evaluate the effectiveness of interventions in real-life routine practice conditions) are becoming increasingly important, as they yield results that can be generalized and applied in routine practice settings, whereas more standard explanatory trials (to evaluate 
whether an intervention works under optimal situations) often fail to be broadly generalizable. ${ }^{72}$ However, many of the issues faced in reporting RCTs also relate to other evaluation designs. ${ }^{73}$ Determining the effectiveness of interventions is very important for policy and practice decision-making, but poor reporting limits the ability to replicate findings, combine evidence in systematic reviews and utilize findings in policy and practice. Therefore, psychosocial intervention studies could consider adhering to the extension of the CONSORT criteria, the CONSORT for Social and Psychological Interventions (CONSORT-SPI). ${ }^{73}$ These criteria could improve the accuracy, comprehensiveness, and transparency of study reports.

Second, some important aspects of psychosocial RCTs are not addressed in the CONSORT statement, e.g. adequate reporting of treatment integrity. It is important to understand how psychosocial interventions are actually delivered and received. Performing a process evaluation alongside an effectiveness trial can improve clarity of possible sources of bias, which allows readers to better assess and interpret treatment effects and generalizability of results and explain why an intervention is effective in one setting and not in another. ${ }^{51}$

Third, RCTs investigating caregiver support often struggle with demonstrating quantitative results, while qualitative results show very positive intervention experiences. ${ }^{5}$ This suggests that there is some room for improvement when it comes to appropriate outcome measures for this type of research. Outcome measures for interventions are often used based on their psychometric properties or familiarity in the field, but the results of this thesis may argue that outcome measures tailored to the intervention content and the personal situation of the target audience could yield more positive results. For example instruments like Goal Attainment Scaling enable identification of individual benefits beyond the generalized measures. ${ }^{74}$ However, this recommendation can be debated, as the ultimate aim of interventions is to improve the overall quality of life, which can be demonstrated with more generic instruments. Furthermore, statistically significant benefits on retrospective self-report measures may be marginal in clinical practice. Therefore, measures like the Experience Sampling Method (ESM) could be a useful addition to measure variations in intervention outcomes in every-day-life situations, possibly mapping individualized evidence of intervention benefit more accurately than retrospective self-report measures only administered pre- and post-intervention. ${ }^{75,76}$

Last, future research could consider tailoring the content of support interventions like Partner in Balance to specific subgroups of caregivers, such as 
caregivers of people with young-onset dementia and children to further optimize the program's effectiveness. Also, caregiver needs may depend on the relationship with the person with dementia, thus intervention effects may vary between for example spouses and adult children. Future research should consider including larger samples to make valid statements about the benefits for these subgroups of caregivers. Furthermore, sustainability of long-term intervention effects should be evaluated and a cost-consequence or cost-effectiveness analysis should be conducted to inform decision makers on the value of Partner in Balance.

In conclusion, the research presented in this thesis provides a valuable contribution to the growing knowledge on e-health for dementia caregivers and showed that a tailored support intervention for dementia caregivers in the early stages is effective in increasing caregiver self-efficacy. Results can be used to inform clinicians and researchers of the delivery and effects of Partner in Balance as a tool to support dementia caregivers. It can be concluded that it is important to take both the benefits and the costs of caregiving into account in intervention development and research. 


\section{References}

1. Ramakers IH, Visser PJ, Aalten P, Kester A, Jolles J, Verhey FR. Affective symptoms as predictors of Alzheimer's disease in subjects with mild cognitive impairment: a 10-year follow-up study. Psychol Med 2010; 40(7): 1193-201.

2. Blieszner R, Roberto KA. Care partner responses to the onset of mild cognitive impairment. Gerontologist 2010; 50(1): 11-22.

3. Gaugler JE, Kane RL, Kane RA, Newcomer R. Early community-based service utilization and its effects on institutionalization in dementia caregiving. Gerontologist 2005; 45(2): 177-85.

4. Kjallman Alm A, Hellzen O, Norbergh KG. Experiences of long term ongoing structured support in early stage of dementia - a case study. Int $J$ Older People Nurs 2013.

5. Banningh LW, Vernooij-Dassen MJ, Vullings M, Prins JB, Rikkert MG, Kessels RP. Learning to live with a loved one with mild cognitive impairment: effectiveness of a waiting list controlled trial of a group intervention on significant others' sense of competence and well-being. Am J Alzheimers Dis Other Demen 2013; 28(3): 228-38.

6. de Vugt ME, Verhey FR. The impact of early dementia diagnosis and intervention on informal caregivers. Prog Neurobiol 2013; 110: 54-62.

7. Ducharme F, Dube V, Levesque L, Saulnier D, Giroux F. An Online Stress Management Training Program as a Supportive Nursing Intervention for Family Caregivers of an Elderly Person. canadian nursing informatics journal 2011; 6(2): 13-44.

8. Andersson G, Cuijpers P. Internet-based and other computerized psychological treatments for adult depression: a meta-analysis. Cognitive Behaviour Therapy 2009; 38: 196-205.

9. Tang WK, Chan CY. Effects of psychosocial interventions on self-efficacy of dementia caregivers: a literature review. Int J Geriatr Psychiatry 2015.

10. Olazaran J, Reisberg B, Clare L, et al. Nonpharmacological therapies in Alzheimer's disease: a systematic review of efficacy. Dement Geriatr Cogn Disord 2010; 30(2): 161-78.

11. Clement $S$, Schauman $\mathrm{O}$, Graham $\mathrm{T}$, et al. What is the impact of mental health-related stigma on help-seeking? A systematic review of quantitative and qualitative studies. Psychol Med 2015; 45(1): 11-27. 
12. Greenwood N, Smith R. Barriers and facilitators for male carers in accessing formal and informal support: A systematic review. Maturitas 2015; 82(2): 1629.

13. Beck AT. Cognitive therapy and the emotional disorders: Penguin; 1979.

14. Bless H, Fiedler K, Strack F. Social cognition: How individuals construct social reality: Psychology Press; 2004.

15. Drentea P, Clay OJ, Roth DL, Mittelman MS. Predictors of improvement in social support: Five-year effects of a structured intervention for caregivers of spouses with Alzheimer's disease. Soc Sci Med 2006; 63(4): 957-67.

16. Boots LM, de Vugt ME, van Knippenberg RJ, Kempen GI, Verhey FR. A systematic review of Internet-based supportive interventions for caregivers of patients with dementia. Int J Geriatr Psychiatry 2014; 29(4): 331-44.

17. Dam A, de Vugt ME, Klinkenberg IP, Verhey FR, van Boxtel MP. A systematic review of social support interventions for caregivers of people with dementia: Are they doing what they promise? Maturitas 2016.

18. Frijda $\mathrm{NH}$, Kuipers $\mathrm{P}$, Schure E. Relations among emotion, appraisal, and emotional action readiness. Journal of Personality Social Psychol 1989; 57: 212-28.

19. Wolfs CA, de Vugt ME, Verkaaik M, Verkade PJ, Verhey FR. Empowered or overpowered? Service use, needs, wants and demands in elderly patients with cognitive impairments. Int J Geriatr Psychiatry 2010; 25(10): 1006-12.

20. Roelands M, Van Oost P, Depoorter A. Service use in family caregivers of persons with dementia in Belgium: psychological and social factors. Health Soc Care Community 2008; 16(1): 42-53.

21. Wolfs $\mathrm{CA}$, de Vugt ME, Verkaaik M, et al. Rational decision-making about treatment and care in dementia: A contradiction in terms? Patient Educ Couns 2011.

22. Fredrickson BL. The broaden-and-build theory of positive emotions. Philos Trans R Soc Lond B Biol Sci 2004; 359(1449): 1367-78.

23. Huber M, Knottnerus JA, Green L, et al. How should we define health? Bmj 2011; 343: d4163.

24. von Faber M, Bootsma-van der Wiel A, van Exel E, et al. Successful aging in the oldest old: Who can be characterized as successfully aged? Arch Intern Med 2001; 161(22): 2694-700. 
25. Carbonneau H, Caron C, Desrosiers J. Development of a conceptual framework of positive aspects of caregiving in dementia. Dementia 2010; 9(3): 327-53.

26. Lazarus R, Folkman S. Stress, appraisal and coping. New York: Springer; 1984.

27. A. B. Self-Efficacy: The Exercise of Control. . New York: W.H. Freeman and Company; 1997.

28. Acton GJ, Kang J. Interventions to reduce the burden of caregiving for an adult with dementia: a meta-analysis. Res Nurs Health 2001; 24(5): 349-60.

29. Toms GR, Quinn C, Anderson DE, Clare L. Help yourself: perspectives on self-management from people with dementia and their caregivers. Qual Health Res 2015; 25(1): 87-98.

30. Lorig KR, Ritter PL, Dost A, Plant K, Laurent DD, McNeil I. The Expert Patients Programme online, a 1-year study of an Internet-based selfmanagement programme for people with long-term conditions. Chronic IIIn 2008; 4(4): 247-56.

31. Forducey PG, Glueckauf RL, Bergquist TF, Maheu MM, Yutsis M. Telehealth for persons with severe functional disabilities and their caregivers: facilitating self-care management in the home setting. Psychol Serv 2012; 9(2): 144-62.

32. Serafini JD, Damianakis T, Marziali E. Clinical practice standards and ethical issues applied to a virtual group intervention for spousal caregivers of people with Alzheimer's. Soc Work Health Care 2007; 44(3): 225-43.

33. Barlow J, Wright C, Sheasby J, Turner A, Hainsworth J. Self-management approaches for people with chronic conditions: a review. Patient Educ Couns 2002; 48(2): 177-87.

34. van der Vaart R, Witting M, Riper H, Kooistra L, Bohlmeijer ET, van GemertPijnen LJEWC. Blending online therapy into regular face-to-face therapy for depression: content, ratio and preconditions according to patients and therapists using a Delphi study. BMC Psychiatry 2014; 14(355).

35. Wilhelmsen M, Lillevoll K, Risør MB, et al. Motivation to persist with internetbased cognitive behavioural treatment using blended care: a qualitative study. BMC Psychiatry 2013; 13(296).

36. Glueckauf RL, Ketterson TU, Loomis JS, Dages P. Online support and education for dementia caregivers: overview, utilization, and initial program evaluation. Telemed J E Health 2004; 10(2): 223-32. 
37. Coulehan MB. Impact of a Web-Based Educational and Peer-Support Intervention for Dementia Caregivers. Aging in America Conference American Society on Aging 2011.

38. Craig $\mathrm{P}$, Dieppe $\mathrm{P}$, Macintyre $\mathrm{S}$, et al. Developing and evaluating complex interventions: the new Medical Research Council guidance. Bmj 2008; 337: a1655.

39. Ruwaard J, Kok RN. Wild West eHealth: Time to hold our horses? The European Health Psychologist 2015; 17(1): 45-9.

40. AARP. Appendix A: Detailed Methodology. Washington D.C., 2004.

41. Brodaty $\mathrm{H}$, Thomson C, Thompson C, Fine M. Why caregivers of people with dementia and memory loss don't use services. Int J Geriatr Psychiatry 2005; 20(6): 537-46.

42. Millenaar JK, de Vugt ME, Bakker C, et al. The Impact of Young Onset Dementia on Informal Caregivers Compared with Late Onset Dementia: Results from the NeedYD Study. Am J Geriatr Psychiatry 2015.

43. Brown RM, Brown SL. Informal caregiving: a reappraisal of effects on caregivers. Social Issues and Policy Review 2014; 8(1): 74-102.

44. Trappenburg J, Jonkman N, Jaarsma $\mathrm{T}$, et al. Self-management: one size does not fit all. Patient Educ Couns 2013; 92(1): 134-7.

45. Moncher FJ, Prinz RJ. Treatment fidelity in outcome studies. Clinical Psychology Review 1991; 11(3): 247-66.

46. Daniels R, van Rossum E, Metzelthin S, et al. A disability prevention programme for community-dwelling frail older persons. Clin Rehabil 2011; 25(11): 963-74.

47. Lorig KR, Holman $H$. Self-management education: history, definition, outcomes, and mechanisms. Ann Behav Med 2003; 26(1): 1-7.

48. Hawe P, Shiell A, Riley T. Complex interventions: how "out of control" can a randomised controlled trial be? Bmj 2004; 328(7455): 1561-3.

49. Greene JC, Yedidia MJ. Provider behaviors contributing to patient selfmanagement of chronic illness among underserved populations. Journal of Health Care for the Poor and Underserved 2005; 16(4): 808-24.

50. Baur A, Fehr J, Mayer C, Pawlu C, Schaudel F. Health care beyond medicine: meeting the demands for new forms of care. Health International, 2011. http://www.mckinsey.com/ /media/mckinsey/dotcom/client_service/pharma and medical products/pmp new/pdfs/healthcare_beyond_medicine.ashx 
51. Ladd BO, McCrady BS, Manuel JK, Campbell W. Improving the quality of reporting alcohol outcome studies: effects of the CONSORT statement. Addictive behaviors 2010; 35(7): 660-6.

52. M. L. Self-conscious emotions: Embarassment, pride, shame, and guilt. . Handbook of emotions. New York: Guilford Press; 1993: pp. 563-73.

53. Hattink B, Meiland F, van der Roest $\mathrm{H}$, et al. Web-Based STAR E-Learning Course Increases Empathy and Understanding in Dementia Caregivers: Results from a Randomized Controlled Trial in the Netherlands and the United Kingdom. J Med Internet Res 2015; 17(10): e241.

54. Blom MM, Zarit SH, Groot Zwaaftink RB, Cuijpers P, Pot AM. Effectiveness of an Internet intervention for family caregivers of people with dementia: results of a randomized controlled trial. PLoS One 2015; 10(2): e0116622.

55. Hayden LJ, Glynn SM, Hahn TJ, Randall F, Randolph E. The Use of Internet Technology for Psychoeducation and Support With Dementia Caregivers. Psychol Serv 2012; 9(2): 215-8.

56. Kajiyama B, Thompson LW, Eto-Iwase T, et al. Exploring the effectiveness of an internet-based program for reducing caregiver distress using the iCare Stress Management e-Training Program. Aging Ment Health 2013; 17(5): 54454.

57. Cristancho-Lacroix V, Wrobel J, Cantegreil-Kallen I, Dub T, Rouquette A, Rigaud AS. A web-based psychoeducational program for informal caregivers of patients with Alzheimer's disease: a pilot randomized controlled trial. J Med Internet Res 2015; 17(5): e117.

58. van der Roest HG, Meiland FJM, Jonker C, Droes RM. User evaluation of the DEMentia-specific Digital Interactive Social Chart (DEM-DISC). A pilot study among informal carers on its impact, user friendliness and, usefulness. Aging Ment Health 2010; 14(4): 461-70.

59. Greene JC, Caracelli VJ, Graham WF. Toward a conceptual framework for mixed-method evaluation designs. Educational Evaluation and policy analysis 1989; 11(3): 255-74.

60. Moore GF, Audrey S, Barker M, et al. Process evaluation of complex interventions: Medical Research Council guidance. Bmj 2015; 350: h1258.

61. Mayo-Wilson E, Grant S, Hopewell S, Macdonald G, Moher D, Montgomery P. Developing a reporting guideline for social and psychological intervention trials. Trials 2013; 14: 242. 
62. Hesser H, Weise C, Rief W, Andersson G. The effect of waiting: A metaanalysis of wait-list control groups in trials for tinnitus distress. J Psychosom Res 2011; 70(4): 378-84.

63. Campbell M, Fitzpatrick R, Haines A, et al. Framework for design and evaluation of complex interventions to improve health. Bmj 2000; 321(7262): 694-6.

64. Boots LMM, de Vugt ME, Smeets CMJ, Kempen GIJM, Verhey FRJ. Validity and implementation of the blended care self-management program 'Partner in Balance' for caregivers of people with early-stage dementia: a process evaluation. In preparation 2016.

65. Pinquart M, Sorensen S. Helping caregivers of persons with dementia: which interventions work and how large are their effects? Int Psychogeriatr 2006; 18(4): 577-95.

66. Kim H. Understanding Internet Use Among Dementia Caregivers: Results of Secondary Data Analysis Using the US Caregiver Survey Data. Interactive journal of medical research 2015; 4(1): e1.

67. Eastman JK, lyer R. The elderly's uses and attitudes towards the Internet. $J$ Consum Marketing 2004; 21: 208-20.

68. Spijker A, Vernooij-Dassen M, Vasse $E$, et al. Effectiveness of nonpharmacological interventions in delaying the institutionalization of patients with dementia: a meta-analysis. J Am Geriatr Soc 2008; 56(6): 1116-28.

69. Zarit S, Femia E. Behavioral and psychosocial interventions for family caregivers. Am J Nurs 2008; 108(9 Suppl): 47-53; quiz

70. Garand L, Dew MA, Eazor LR, DeKosky ST, Reynolds CF, 3rd. Caregiving burden and psychiatric morbidity in spouses of persons with mild cognitive impairment. Int J Geriatr Psychiatry 2005; 20(6): 512-22.

71. Wolfs CA, Kessels A, Severens JL, et al. Predictive factors for the objective burden of informal care in people with dementia: a systematic review. Alzheimer Dis Assoc Disord 2012; 26(3): 197-204.

72. Patsopoulos NA. A pragmatic view on pragmatic trials. Dialogues Clin Neurosci 2011; 13(2): 217-24.

73. Montgomery P, Grant S, Hopewell S, et al. Protocol for CONSORT-SPI: an extension for social and psychological interventions. Implement Sci 2013; 8: 99. 
74. Bouwens SF, van Heugten CM, Verhey FR. The practical use of goal attainment scaling for people with acquired brain injury who receive cognitive rehabilitation. Clin Rehabil 2009; 23(4): 310-20.

75. Greenhalgh T, Howick J, Maskrey N, Evidence Based Medicine Renaissance G. Evidence based medicine: a movement in crisis? Bmj 2014; 348: g3725.

76. van Knippenberg RJM, de Vugt ME, Ponds RW, Myin-Germeys I, van Twillert $B$, Verhey FRJ. Dealing with daily challenges in dementia (Deal-id study): An innovative approach to assess caregiver functioning in the flow of daily life. (submitted) 2016. 


SUMMARY 
Dementia is a generic term for deterioration in memory and other cognitive functions that facilitate a person's ability to function in everyday life. The majority of people with dementia are living at home and cared for by one or more family members, often referred to as the informal caregivers. Caregiving generally puts a large strain on family members, increasing their risk for depression and anxiety. Many caregiver support interventions have been developed to ameliorate negative caregiver consequences. Timing of these interventions might be crucial in maximizing their effects. Early support could help caregivers to adapt to the changes of early dementia and to feel more competent towards care. However, the current contrast between highly advanced tools with respect to the timely diagnosis of dementia and the lack of available support to match this early diagnosis is challenging. Nonetheless, the scientific evidence for early-stage caregiver interventions is not yet conclusive. Next to intervention timing, intervention delivery can also be essential to maximize intervention effects. E-health technology offers an opportunity for accessible tailored interventions. However, existing e-health programs for dementia caregivers are mainly aimed at dealing with dementia related problems in the later stages of dementia, which may not benefit caregivers in the early stages. Therefore, the main aim of this thesis was to develop and evaluate an e-health selfmanagement intervention for caregivers of people with early-stage dementia.

Chapter 1 provides a general introduction to the theme and aims of this thesis. Information on dementia, informal caregiving, and the relevance of caregiver interventions is provided, as well as the research questions and outline of the thesis.

In Chapter 2 a systematic review of the effectiveness, feasibility, and quality of Internet interventions for informal caregivers of people with dementia is presented. Intervention types, dosage and duration differed widely, as did the methodological quality of the 12 included studies. The overall level of evidence was low. However, the results demonstrated that Internet interventions for informal dementia caregivers can improve various aspects of caregiver well-being, e.g., confidence, self-efficacy, stress, burden and depression, provided they comprise multiple components and are tailored to the individual. Furthermore, caregivers could benefit from guidance by a coach or contact with other caregivers.

Chapter 3 describes an exploration of the needs, perceptions and experiences with informal support from the perspective of the primary caregiver and his/her social network members. Results revealed that there is a mismatch between supply and demand of informal support, strengthened by a cognitive bias; primary 
caregivers reported to think for other social network members and vice versa. Both presented unverified and sometimes incorrect thoughts concerning each other's intentions, resulting in not explicitly communicating support wishes and opportunities. Openness in communication might repair this mismatch.

In Chapter 4 an in-depth analysis of the problems, needs and wishes of caregivers of people with early-stage dementia and their perspectives on need for care during these early stages is presented. The results showed that early-stage caregivers struggled with acknowledging needs due to fear of stigma and low acceptance. They stressed the importance of acceptance as a prerequisite for adequate adaptation, but were hindered by lack of knowledge, difficulty acknowledging changes and focus on loss. In contrast, better understanding of the disease, increasing personal time, structuring one's day and using appropriate humor can reduce negative communication. In turn, this may increase positive encounters and caregiver-confidence, contributing to positive interaction with the care recipient and an increase in well-being.

Chapter 5 describes the development of the blended care self-management program 'Partner in Balance' and an evaluation of its feasibility and preliminary effects on self-efficacy and goal attainment. 'Partner in Balance' is a selfmanagement intervention, which means that participants are motivated to reflect on their situation, create personal goals and search for possible solutions. The intervention is presented in a 'blended care' format; it combines online modules with face-to-face contact moments. In the intake session participants and coaches set goals and choose 4 out of 9 available modules or themes that fit their personal situation. Modules consist of information, examples, and personal assignments. Participants follow the modules online at their own convenience, guided by the personal coach. A discussion forum was added to allow caregivers to exchange information and tips. Following the online period the modules and goals are evaluated in a closing-out session with the coach. The tailored intervention approach appeared to be feasible for caregivers in the early stages and supported them in the difficulties of caregiving. The convenience of completing the program at home, the tailored content, and the guidance (both face-to-face and online) were appraised positively. Feedback of the participants was used to increase the program's userfriendliness. Preliminary effects on caregiver self-efficacy and goal attainment were promising. 
Chapter 6 describes the design of a randomized controlled trial evaluating the process characteristics and effects of 'Partner in Balance' on self-efficacy, mastery, quality of life and psychological complaints (depression, anxiety, and stress) compared to a waiting-list control condition receiving usual care.

Chapter 7 assesses the internal and external validity of the 'Partner in Balance' intervention and its implementation in a process evaluation. Data were evaluated from the perspective of both family caregivers (participants) and professionals delivering the intervention (coaches) with the objective to increase the understanding of the mode of delivery. Participation rate of recruited caregivers was $52 \%$. Recruitment barriers reported by coaches were e.g. no computer, no need for intervention or additional support, which were also reasons to decline participation. Younger age and being employed were considered recruitment facilitators. However, caregivers of people with young-onset dementia and children had difficulties identifying with examples about older caregivers. Both participants and coaches mentioned that their relationship with each other had deepened. Participant compliance to all 4 modules was high $(87.8 \%)$ and performance according to protocol as rated by the coaches was substantial $(77 \%)$. The varying intervention period and different dose (i.e. time spent on the intervention) may influence the effectiveness of the intervention. Participants appreciated the personal touch and the confirmation of existing thoughts they experienced during the program. Overall, caregivers attained their previously set goals at the end of the program. Participants reported that the blended structure increased their openness during the online modules. The program's primary focus on the caregiver (and not on the caregiver-patient dyad) was appreciated less. The discussion forum was hardly used. Partner in Balance was evaluated within multiple organizations with coaches from different backgrounds, who received training and supervision in self-management and blended care. A relatively high response rate of organizations to adopt the program was found (73\%). However, a lack of financing and time could hamper the implementation.

Chapter 8 investigates whether 'Partner in Balance' is superior to the waitinglist control condition as evidenced by improved self-efficacy, mastery, quality of life, and lower levels of psychological complaints (depression, anxiety, and stress). The results demonstrated a significant increase in self-efficacy, mastery, and quality of life in the intervention group (Partner in Balance), compared with the control group. No significant differences were found between the groups with respect to depressive symptoms, general distress and perceived stress. 
In Chapter 9 the main results and their scientific interpretation are discussed, together with a reflection on methodological issues and the implications for clinical practice and future research. 



\section{SAMENVATTING}


Dementie is een algemene term voor achteruitgang van het geheugen en andere cognitieve functies die men nodig heeft om te functioneren in het dagelijks leven. De meerderheid van de mensen met dementie woont thuis en wordt verzorgd door een of meerdere familieleden, ook wel mantelzorgers genoemd. Zorgen voor iemand met dementie legt een grote druk op mantelzorgers en verhoogt hun risico op depressieen angstklachten. Om deze negatieve gevolgen te verminderen zijn er ondersteuningsmogelijkheden of interventies voor mantelzorgers ontwikkeld. De timing waarop deze interventies worden ingezet kan cruciaal zijn om hun effect te vergroten. Wanneer familieleden in een vroeg stadium ondersteuning krijgen bij het aanpassen aan de veranderingen die dementie teweeg brengt, kan dit ervoor zorgen dat ze zich meer competent voelen als mantelzorger. Het is echter vreemd dat de nieuwe geavanceerde methoden om een vroegtijdige dementie diagnose te stellen niet samengaan met ondersteuningsmogelijkheden voor mensen die deze vroege diagnose te horen krijgen. Daarentegen is nut van zulke ondersteuningsmogelijkheden in een vroeg stadium nog niet eenduidig wetenschappelijk aangetoond.

Naast timing van een interventie kan de manier waarop een interventie wordt aangeboden ook van essentieel belang zijn om de effecten te vergroten. E-health technologie maakt het mogelijk om op een toegankelijke manier op maat gemaakte interventies aan te bieden. Reeds bestaande e-health interventies voor mantelzorgers van mensen met dementie zijn voornamelijk gericht op het omgaan met dementie-gerelateerde problemen die vaak kenmerkend zijn voor de latere stadia van dementie en mogelijk niet passen bij de situatie van mantelzorgers in een vroeg stadium. Daarom richtte het onderzoek in dit proefschrift zich op het ontwikkelen en evalueren van een e-health interventie voor mantelzorgers van mensen met dementie in een vroeg stadium.

Hoofdstuk 1 geeft een algemene inleiding op het thema en de doelstellingen van het proefschrift. In dit hoofdstuk komen dementie, mantelzorg en de relevantie van mantelzorginterventies aan bod. Ook worden de onderzoeksvragen en de opzet van dit proefschrift besproken.

In Hoofdstuk 2 wordt een literatuurstudie gepresenteerd die zich richt op de effectiviteit, bruikbaarheid en kwaliteit van e-health interventies voor mantelzorgers van mensen met dementie. De invulling per interventie en de methodologische kwaliteit van de 12 besproken studies liepen sterk uiteen. Het bewijs van effectiviteit van de verschillende interventies bleek over het algemeen laag te zijn. De resultaten 
suggereerden wel dat e-health interventies voor mantelzorgers van mensen met dementie een positief effect kunnen hebben op verschillende aspecten van hun welzijn (onder anderen hun zelfvertrouwen, zelf-effectiviteit, stress, belasting en depressie), mits deze interventies bestonden uit meerdere componenten en waren afgestemd op het individu. Daarnaast bleek dat contact (op afstand) met een coach en andere mantelzorgers een belangrijke toevoeging kan zijn.

Hoofdstuk 3 beschrijft een onderzoek naar de behoeften, percepties en ervaringen met informele steun vanuit het perspectief van de primaire mantelzorger en zijn of haar sociale netwerk. Uit de resultaten bleek dat er een slechte afstemming is tussen vraag en aanbod van informele steun, wat mogelijk wordt veroorzaakt door een bepaalde denkfout. Mantelzorgers vullen vaak gedachten in voor de leden uit hun sociale netwerk en andersom. Zowel mantelzorgers als hun netwerkleden rapporteerden gedachten over elkaars bedoelingen die niet bij elkaar werden geverifieerd en soms onjuist waren. Dit resulteerde in een onduidelijke communicatie over de wensen en mogelijkheden op het gebied van wederzijdse ondersteuning. Meer openheid in de communicatie zou deze slechte afstemming of 'mismatch' kunnen herstellen.

In hoofdstuk 4 worden de problemen, behoeften en wensen van mantelzorgers van mensen met dementie in een vroeg stadium onderzocht. Daarnaast wordt hun behoefte aan zorg in dit vroege stadium in kaart gebracht. De resultaten toonden aan dat mantelzorgers in een vroeg stadium moeite hebben met het erkennen van hun behoeften, mogelijk door een bepaalde angst voor stigmatisering en problemen met acceptatie. Mantelzorgers benadrukten het belang van acceptatie als voorwaarde om zich te kunnen aanpassen aan de nieuwe situatie, maar ze werden in hun acceptie beperkt door een gebrek aan kennis, moeite met het erkennen van veranderingen en een overheersende focus op verlies. Daarentegen kan meer begrip van de ziekte, meer tijd voor zichzelf, een bepaalde structuur in de dag en gepaste humor de negatieve communicatie tussen de mantelzorger en de persoon met dementie verminderen. Dit kan het aantal positieve momenten en het zelfvertrouwen van de mantelzorger verhogen. Uiteindelijk dan dit weer bijdragen aan een positievere interactie met de persoon met dementie en een betere kwaliteit van leven.

Hoofdstuk 5 beschrijt de ontwikkeling van de interventie 'Partner in Balans' en een evaluatie van de bruikbaarheid en de voorlopige effecten van het programma op zelf-effectiviteit (zelfvertrouwen) van de mantelzorger en het bereiken van doelen. 
'Partner in Balans' is een zelfmanagementinterventie, wat betekent dat deelnemers worden aangezet om na te denken over persoonlijke doelen en actieplannen om deze doelen te bereiken. De interventie wordt gegeven in een zogenaamde 'blended' vorm, waarbij online modules worden gecombineerd met face-to-face gesprekken. In een eerste gesprek worden doelen gesteld en kiezen deelnemers samen met hun coach 4 uit de 9 beschikbare modules of thema's die bij hun situatie passen. Modules bestaan uit informatie, voorbeelden en persoonlijke opdrachten. Deelnemers kunnen de modules thuis in hun eigen tijd bekijken, waarbij ze op afstand worden ondersteund door de coach. Daarnaast is er een discussieforum beschikbaar waar deelnemers met andere mantelzorgers tips of ervaringen kunnen uitwisselen. Na de online periode worden de modules en doelen geëvalueerd in een afsluitend gesprek met de coach. De op maat gesneden interventie bleek bruikbaar voor mantelzorgers van mensen met dementie in een vroeg stadium en ondersteunde hen in het zorgen voor hun familielid. Het gemak om het programma thuis te kunnen volgen, de op maat gemaakte inhoud en de begeleiding van de coach (face-to-face en online) werden positief beoordeeld. De ervaringen van de mantelzorgers werden gebruikt om het programma gebruikersvriendelijker te maken. De studie toonde veelbelovende voorlopige effecten op zelf-effectiviteit en het bereiken van doelen.

Hoofdstuk 6 beschrijft de opzet van een gerandomiseerde gecontroleerde studie waarin zowel de proceskenmerken als de effecten van 'Partner in Balans' op zelf-effectiviteit, ervaren controle, kwaliteit van leven en psychische klachten (depressie, angst en stress) worden vergeleken met een wachtlijst controlegroep.

Hoofdstuk 7 beoordeelt de interne en externe validiteit van 'Partner in Balans' en de implementatie van het programma in een procesevaluatie. De gegevens werden geëvalueerd vanuit het perspectief van zowel de mantelzorgers (deelnemers) als de professionals (dat wil zeggen: de coaches die die de interventie hebben aanboden en begeleid). Van de mantelzorgers die gevraagd werden om deel te nemen heeft $52 \%$ daadwerkelijk deelgenomen. Factoren die de werving bemoeilijkten waren het niet in bezit zijn van een computer of het geen behoefte hebben aan een interventie of extra steun. Werving werd gemakkelijker wanneer mantelzorgers relatief jonge waren en/of nog werkzaam waren. Zowel partners als kinderen van mensen met dementie op jonge leeftijd hadden echter moeite om zich te identificeren met de gegeven voorbeelden van oudere mantelzorgers. De deelnemers en de coaches gaven aan dat hun onderlinge relatie was verdiept na 
deelname aan 'Partner in Balans'. Het percentage van deelnemers die alle 4 de modules had afgerond was hoog $(87,8 \%)$. Daarnaast werd het programma volgens de coaches grotendeels volgens protocol gegeven (77\%). De variërende interventieperiode en dosering (aantal gevolgde modules en tijd die er aan werd besteed) kan de effectiviteit van de interventie hebben beïnvloed. Mantelzorgers waardeerden het persoonlijke aspect en de bevestiging die het programma ze gaf. Over het algemeen bereikten de mantelzorgers hun vooraf gestelde doelen na afloop van het programma. Mantelzorgers gaven aan dat de 'blended' aanpak hun openheid tijdens de online modules vergrootte. Ze waren minder enthousiast over het feit dat de interventie zich alleen op de partner richt en niet ook op de persoon met dementie. Het discussieforum werd amper gebruikt. Partner in Balans werd geëvalueerd binnen meerdere organisaties, met coaches van verschillende achtergronden. Alle coaches ontvingen scholing en begeleiding in zelfmanagement en het geven van 'blended' zorg.

Hoofdstuk 8 onderzoekt of 'Partner in Balans' beter is dan een wachtlijst controle conditie op het gebied van zelf-effectiviteit, ervaren controle, kwaliteit van leven en psychische klachten (depressie, angst en stress). De resultaten toonden een significante toename van zelf-effectiviteit, ervaren controle en kwaliteit van het leven in de interventiegroep (Partner in Balans), in vergelijking met de controlegroep. Er werden geen significante verschillen gevonden tussen de groepen voor wat betreft depressieve symptomen, angst en ervaren stress.

In hoofdstuk 9 worden de belangrijkste resultaten en hun wetenschappelijke interpretatie besproken, naast een kritische reflectie op methodologische aspecten en de implicaties voor de klinische praktijk en toekomstig onderzoek. 

KNOWLEDGE VALORIZATION 
This thesis described the development and evaluation of the supportive e-health intervention 'Partner in Balance' for caregivers of people with early-stage dementia. In this valorization paragraph we aim to describe how the obtained knowledge from our research can be made valuable in social and economical contexts.

\section{Relevance and innovation}

The global cost of dementia was estimated at 604 billion dollars in the 2011 World Alzheimer Report. ${ }^{1}$ Due to the rise of people with dementia in the near future, it is expected that the cost of care for dementia will increase by $85 \%$ in 2030 , possibly making it the most expensive disease of our society. ${ }^{1}$ Efficacious pharmacotherapies are not yet available to cure dementia, but previous studies showed that psychosocial interventions aimed at reducing caregiver burden and increasing their self-efficacy might reduce (indirect) dementia care costs, as they improve caregiver mood, caregiver quality of life and delay the institutionalization of the care recipient for up to 1.5 years. ${ }^{2-4}$ These results are noteworthy, considering that a single day at a nursing home costs approximately 200 Dollars in the US. ${ }^{5}$

Most caregiver interventions focus on the mild to moderate stages of dementia, but timing of these interventions might be crucial in maximizing the effects. Our results showed that early support could help caregivers adapt to the changes of early dementia and feel more competent to care. Next to intervention timing, intervention delivery can also be essential to maximize intervention effects. E-health technology offers an opportunity for easy accessible and tailored interventions and could be an efficient alternative to continue to provide education and support for the informal caregivers at reduced costs. ${ }^{6-8}$ Our literature review confirmed this by presenting the promising results Internet interventions for dementia caregivers have on caregiver confidence, self-efficacy, stress, burden, and depression. However, existing e-health programs for dementia caregivers are mainly aimed at dealing with dementia related problems in the later stages of dementia, and results of our needs assessment showed that support should fit the stage-specific needs of the caregiver. E-health self-management interventions could suit the caring role transition caregivers face during the early stages and help future informal caregivers cope with the challenges of caring for a person with dementia. ${ }^{9-10}$ The studies in this thesis confirmed this hypothesis, as the newly developed e-health self-management intervention for caregivers of people with early-stage dementia 'Partner in Balance' is effective in increasing dementia caregiver self-efficacy, mastery, and quality of life. 


\section{Target groups}

The findings in this thesis are relevant for caregivers of people with early-stage dementia, dementia healthcare professionals, and policy makers.

Caregivers of people with early-stage dementia can profit from our research as the 'Partner in Balance' program effectively increased caregivers' self-efficacy, mastery and quality of life. Dementia healthcare professionals could consider online support for informal dementia caregivers in addition to the already provided care as usual. In their care considerations they could take the stage of the disease, individual characteristics and coping mechanism of the caregiver, and relationship with the care recipient prior to the disease into account and adapt the care accordingly to suit the situation and needs of the individual caregiver. Furthermore, the results show that many difficulties in the early stages can be attributed to difficulties accepting the changes in family roles and relationships, therefore targeting acceptance of these changes in early interventions such as the currently developed program 'Partner in Balance' might prove to be beneficial. During the feasibility and effectiveness study, 'Partner in Balance' was introduced in some institutions in the southern part of the Netherlands. If interested, the institutions could participate in the study, delivering the intervention in their care practice under regular supervision of the research team. The Academic Hospital Maastricht, the Riagg Maastricht, all 5 chains of 'Hulp bij Dementie' (Integrated Dementia Care in Limburg), Elkerliek Hospital Helmond, Catharina Hospital Eindhoven and Metggz Roermond have agreed to participate and already use the program in their daily practice. Results showed that caregivers who followed the program reported an increase in self-efficacy, mastery, and quality of life. As the number of people with dementia will increase in the future, this finding could be of great interest to insurance companies and policy makers. When policy and practice in the formal care system invest in improving the capability of informal carers to help them continue to care for their family member with dementia with effective programs such as 'Partner in Balance', this could contribute to the sustainability of the healthcare for people with dementia and their carers. Furthermore, the policies of the formal care system are conceptualized in rational and general terms, whereas effective care should fit the expectations and experiences of informal family carers that emerge out of a unique set of personal circumstances. If insurance companies and policy makers take these personal circumstances into account this could increase the effectiveness of support for informal carers. 


\section{Activities and products}

Based on the user- and professional input, evidence from previous research, and feasibility findings the online self-management program 'Partner in Balance' was developed. The program comprises of multiple components, is tailored to the individual and includes interaction with a coach and the possibility to interact with other caregivers. The program is tailored to individual differences in caregivers based on their needs, while stressing the positive aspects of caregiving and focusing on adjusting expectations to be able to facilitate acceptance during the early stages.

The different institutions who offered the program during the study period were updated of the study progress through a bi-annual newsletter. Furthermore, we visited Alzheimer Cafes, senior centers and mental health insitutions in the southern part of the Netherlands to present our study and preliminary findings and to ask feedback from both caregivers and professionals.

\section{Schedule and implementation}

As the intervention proved to be effective on caregiver self-efficacy, mastery and quality of life, the institutions that offered the program during the study and followed the coach training will focus on implementing the program in their daily care. Currently, we are exploring the possibilities to implement the program on a larger scale, by focusing on municipalities within the scope of Dementia Friendly Communities. We are currently discussing the option of implementing 'Partner in Balance' within the EU Prevent project (www.euprevent.eu), in which German and French regions have also expressed their interest. We are currently investigating (financial) opportunities for translating and implementing 'Partner in Balance' on a European level. Furthermore, as spousal- and child caregivers of people with youngonset dementia may benefit from other topics and examples, financial means were obtained to develop additional modules to meet the needs of this specific group. 


\section{References}

1. Alzheimer Disease International. World Alzheimer Report. London: ADI, 2011.

2. Van Mierlo LD, Meiland FJ, Van der Roest HG, Droes RM. Personalised caregiver support: effectiveness of psychosocial interventions in subgroups of caregivers of people with dementia. Int J Geriatr Psychiatry 2012; 27(1): 1-14.

3. Selwood A, Johnston K, Katona C, Lyketsos C, Livingston G. Systematic review of the effect of psychological interventions on family caregivers of people with dementia. J Affect Disord 2007; 101(1-3): 75-89.

4. Olazaran J, Reisberg B, Clare L, et al. Nonpharmacological therapies in Alzheimer's disease: a systematic review of efficacy. Dement Geriatr Cogn Disord 2010; 30(2): 161-78.

5. Wimo A, Karlsson G, Winblad B. Health economic aspects of dementia. In: Ames D, Burns A, O’Brien J, eds. Dementia. Boca Raton, FL: CRC Press; 2010: 136-46.

6. Glueckauf RL, Loomis JS. Alzheimer's Caregiver Support Online: Lessons learned, initial findings and future directions. Neurorehabilitation 2003; 18(2): 135-46.

7. Martin-Carrasco M, Martin MF, Valero CP, et al. Effectiveness of a psychoeducational intervention program in the reduction of caregiver burden in Alzheimer's disease patients' caregivers. Int J Geriatr Psychiatry 2009; 24(5): 489-99.

8. Lewis BA, Williams DM, Neighbors CJ, Jakicic JM, Marcus BH. Cost Analysis of Internet vs. Print Interventions for Physical Activity Promotion. Psychol Sport Exerc 2010; 11(3): 246-9.

9. Serafini JD, Damianakis T, Marziali E. Clinical practice standards and ethical issues applied to a virtual group intervention for spousal caregivers of people with Alzheimer's. Soc Work Health Care 2007; 44(3): 225-43.

10. Robinson L, Brittain K, Lindsay S, Jackson D, Olivier P. Keeping In Touch Everyday (KITE) project: developing assistive technologies with people with dementia and their carers to promote independence. Int Psychogeriatr 2009; 21(3): 494-502. 

DANKWOORD 
Onwerkelijk maar waar... het proefschrift is klaar! Wat zijn deze 4 jaar voorbij gevlogen en wat heb ik veel om dankbaar voor te zijn. In dit hoofdstuk wil ik graag alle mensen bedanken die ervoor hebben gezorgd dat dit boekje nu voor jullie ligt. Ga er maar eens goed voor zitten, want dat zijn er een heleboel! :)

Graag wil ik als eerste alle deelnemers bedanken voor hun openheid en enthousiasme voor Partner in Balans. Zonder jullie was er helemaal geen onderzoek om te beschrijven. Natuurlijk ook bedankt voor alle heerlijke kopjes koffie, cappuccino, thee, zelfgebakken wafels, koekjes, vlaai, tompoezen, cakejes, bonbons, vers belegde broodjes en zelfs vijgen uit eigen tuin. Het kostte me de nodige kilo's, maar het was het dubbel en dwars waard! Ik heb ontzettend veel van jullie geleerd en ik hoop dat jullie door deelname aan het onderzoek ook wat extra steun hebben ervaren.

Daarnaast wil ik mijn promotieteam bestaande uit Marjolein de Vugt, Frans Verhey en Ruud Kempen graag hartelijk bedanken!

Frans, jouw persoonlijke betrokkenheid is bewonderenswaardig! Daarnaast ben ik ook heel dankbaar voor je inhoudelijke kennis, je visie op de wetenschap en je humor. Je hebt me geleerd als eerste te kijken naar de mens achter de ziekte en dat zal ik ook altijd blijven doen.

Ruud, je was formeel mijn promotor op afstand maar je was altijd op de hoogte en dacht mee met elke uitdaging of probleem du jour. Jouw kritische blik hielp me telkens weer een stapje verder. Ook heb ik nog nooit een professor gekend die zo snel en uitgebreid feedback geeft op mijn artikels! Ontzettend bedankt, je was een enorme steun.

Marjolein, wij hebben samen het meeste meegemaakt de afgelopen 4 jaar en voor mijn gevoel ben ik onder jouw hoede echt 'opgegroeid' als wetenschapper en lid van het Alzheimer Centrum. Je hebt me heel veel geleerd en me het vertrouwen gegeven dat ik vooral in mezelf moet geloven. Bedankt voor alle kansen die je me hebt gegeven en voor alle interessante maar stiekem ook hele gezellige overleggen die we hebben gehad. Ik ben heel blij met de band die we hebben gekregen en ik hoop dat we elkaar in de toekomst mogen blijven treffen.

Leden van de beoordelingscommissie, hartelijk dank voor het lezen en beoordelen van mijn proefschrift! 
Het onderzoek in dit proefschrift bestond uit verschillende onderdelen waar veel mensen een belangrijke bijdrage aan hebben geleverd. Ramon Daniels, bedankt voor je input tijdens de ontwikkeling van de interventie. Rudolf Ponds, Mirjam Vos, Dymphie in de Braek, Debby Peeters, Susanne Valentijn, Sonja Hutschemakers, Yindee van Os, Floor van Bergen, Barbera van Reijswoud, Liesbeth Joosten, Chantal Geusgens en Martine Josten, bedankt voor het delen van jullie professionele kennis die ik mocht meenemen in Partner in Balans. Alard Weisscher, bedankt voor het prachtige design van Partner in Balans en de folder. Froukje Sleeswijk Visser, bedankt voor je hulp bij het ontwikkelen van het context-mapping materiaal voor de focusgroepen. Ralph Blomberg, bedankt voor het design van mooie waaier, stappenplannen en nieuwsbrieven. Jan Pillaerds en Eric Sol, bedankt voor de ontwikkeling van de website en voor de technische ondersteuning. Paul Bremmers en Ageeth Thalen, bedankt voor jullie hulp bij het mogelijk maken van de focusgroepen in Roermond. Jullie hebben daarna nog meegeholpen met meerdere onderzoeken van het Alzheimer Centrum Limburg en zijn hier altijd met het grootste enthousiasme ingedoken. Hartelijk dank! Agnes Steijnen, bedankt voor de leuke interviews bij Sjpek mit Eier! Frans Gijzelsstichting, Rotary Elsloo en Jacqueline Winter, bedankt voor jullie steun bij het verwezenlijken van onze producten vanuit het onderzoek.

Graag wil ik alle instellingen die hebben meegewerkt aan het onderzoek bedanken voor hun enthousiasme voor Partner in Balans: azM, Riagg Maastricht, Metggz Roermond, Hulp bij Dementie Midden-Limburg, Noord-Limburg, MaastrichtHeuvelland, Parkstad en Westelijke Mijnstreek, Elkerliek Ziekenhuis Helmond en Catharina Ziekenhuis Eindhoven. Het benoemen van de instellingen is eigenlijk niet voldoende, want zonder het enthousiasme van sommige mensen was het misschien wel heel anders gelopen. Ron Colaris, jij bent het boegbeeld van positiviteit! Wat heb ik veel mooie levenslessen van je geleerd en leuke gesprekken met je gehad. Je hebt een zware periode achter de rug, maar richt je blik altijd op de toekomst. Ik hoop dat we nog heel vaak mogen samenwerken. Lisette Dickhoff, Judith Lansink, Nicole Thomas, Henk Derks, Simone Denis, Yindee van Os, Manon Hanssen en Kittie D'Hoine, bedankt dat ik meerdere keren mocht langskomen om Partner in Balans onder de aandacht te brengen. Susanne Valentijn en Harmke Nijboer, bedankt dat jullie Partner in Balans zo snel in jullie hart hebben gesloten en voor jullie geweldige hulp bij de inclusie. 
De mensen die er mede voor hebben gezorgd dat Partner in Balans zo positief werd ervaren zijn de coaches! Lieve Claire Wolfs, Mirjam Vossen, Ria Verstappen, Annie Govers, Karin van de Ven, Inez Ramakers, Kati van Heesch, Nynke de Haan, Hanneke Withagen, Maud Nivo, Rianne van Wijlick, Renske Hamel, Astrid Jennissen, Kelly Thijssen, Charlotte den Butter en Joany Millenaar, jullie waren het hart van de cursus! Bedankt voor jullie deelname aan de training en de geweldige begeleiding van onze deelnemers, vaak zelfs in jullie eigen tijd. Ik ben jullie eeuwig dankbaar! Ria, naast coach was je ook ambassadrice van Partner in Balans en heb je zelfs regelmatig presentaties gegeven uit eigen initiatief! Geen mantelzorger ging aan jou voorbij zonder dat je aan Partner in Balans dacht. Voor mij had je altijd tijd, ook al had je genoeg aan je hoofd. Ontzettend bedankt voor je enthousiasme. Binnenkort doen we echt dat wijntje in de tuin van je nieuwe huisje! Claire, jij was niet alleen coach van Partner in Balans, je hebt me ook geholpen met het focusgroep artikel en je was altijd betrokken en behulpzaam. Ik weet niet hoe je het doet; kliniek, wetenschap en ook nog een klein menneke thuis, je bent een inspiratie! Renske, lieve coach, lieve mede aio-vertegenwoordiger en treinbuddy. Zelfs toen je al lang een nieuwe baan had bleef je coach van Partner in Balans en was je altijd bereikbaar voor mijn noodgevallen. Je bent een geweldige psychologe en een heerlijk mens. Natuurlijk wil ik ook graag alle opgeleide coaches die vol interesse de training hebben gevolgd maar geen geschikte deelnemers konden vinden op dat moment bedanken.

Naast de coaches hebben nog heel veel mensen geholpen met de inclusie van Partner in Balans. Bedankt lieve casemanager/trajectbegeleiders van Hulp bij Dementie, Whitney, Isabelle, Dymphie, Jeanette, Albert, Anita, Marieke, Charlotte, Arthur, Jan, Arlette, Alzheimer Nederland en alle mensen die ik nog niet heb genoemd. Jullie hebben me geweldig geholpen en ik ben jullie eeuwig dankbaar!

Jack Poels, hartelijk dank dat ik je mooie schilderij mocht gebruiken voor de cover en je quote uit Heilige Antonius, die zo treffend is voor dementie. En natuurlijk veel dank aan pap, Hub Boesten en Jan Teunissen voor het regelen!

Mijn eigen stadje Roermond is natuurlijk de mooiste stad van Nederland en dat werd de afgelopen tijd nog eens bevestigd door de werkgroep 'Dementievriendelijk Roermond'. Lieve Lei, Betty, Kees, Ron, Iris en Ingrid, bedankt voor jullie geweldige 
ideeën, enthousiasme en daadkracht. Ik ben erg dankbaar dat ik samen met jullie de start van de beweging Dementievriendelijk Roermond heb mogen vormgeven. Kees, nog eens extra bedankt voor je hulp tijdens de Zumba marathon en studie. Ik ben heel blij dat ik jou en Mary heb leren kennen, jullie hebben echt $\checkmark$ voor Alzheimer!

Een speciaal bedankje wil ik richten aan Marianne Smitsmans voor haar persoonlijke en geweldige inzet voor dementie, mantelzorgers en Roermond in het algemeen.

Geert Leenders, er zijn teveel dingen om op te noemen waar ik je voor kan bedanken! Je hebt me meteen vanaf het begin laten zien dat je als wetenschapper werkt voor de mensen en de maatschappij! Door jou heb ik ontelbaar interessante presentatie-ervaringen, ontmoetingen en connecties opgedaan. Jouw talent om werelden samen te brengen is ongekend. Ik heb diepe bewondering voor alles wat je hebt gedaan en voor je bescheidenheid die je nooit bent verloren. Dankjewel voor alles!

Nico Rozendaal en Ron Mengelers, jullie zijn onmisbaar voor ons allemaal! Ron, bedankt voor je geduld om dagenlang met mij acceptatietesten te doen in alle mogelijke browsers en met 100 nep email-adressen. Samen kregen we er wel eens een punthoofd van, maar door jou bleef ik altijd lachen! Nico, crisismanagement is jouw 'middle name'. Hoe druk je het ook hebt, je hebt altijd alle aandacht en je stopt niet tot het probleem is opgelost. Daarnaast heb je systemen bedacht waar we nu allemaal niet meer zonder kunnen. Er is geen tweede Nico, you are one of a kind! Bedankt!!

Danielle en Els, bedankt voor jullie onmisbare hulp met eigenlijk alles wat we nodig hadden op de afdeling! Ook bedankt voor jullie hulp bij de afronding van de promotie!

Seb, analyseren klonk als een akelig donker gat waar ik me niet aan wilde wagen, maar jouw hulp en uitleg was geweldig. Ik schrijf inmiddels syntaxen en vergelijk modelletjes alsof het niets is. Ontzettend bedankt voor je geduld. Dat je nog maar veel chocola en drop mag ontvangen in de toekomst, want je bent voor iedereen op de afdeling een grote steun! 
Alieske, bedankt dat ik samen met jou een superleuk artikel heb mogen schrijven. Ik heb bewondering voor je werkethiek en weet zeker dat jij en Inlife een geweldige toekomst tegemoet gaan.

Claudia, jij was de motor van het onderzoek. Je had altijd het overzicht, ook op momenten dat ik dat even helemaal kwijt was. Bedankt voor je administratieve talent, alle huisbezoeken en liefde voor de deelnemers. Ik vond het superleuk om met jou samen Partner in Balans te draaien! Inge, je hebt me geweldig geholpen met de start van Partner in Balans en nog lang daarna! Ik gun je al het beste en ben ontzettend blij dat je bij ons bent komen werken. Hanneke, Partner in Balans werd in een korte periode ook jouw kindje, je hebt je met hart en ziel voor de cursus en het onderzoek ingezet en daar ben ik je heel dankbaar voor! Caroline, je had maar weinig tijd voor je stage, maar je stelde jezelf als doel om alles af te ronden voor het einde (wat eigenlijk onbegonnen werk was) en het is je gelukt! Dankjewel voor alles!

Lieve Rosalie, wat hadden deze 4 jaar er anders uitgezien zonder jou. Alles heb ik met jou samen mogen beleven, onze eerste grote presentatie waarbij we meteen 300 mensen mochten toespreken, daarna met gemak elke avond een andere Rotary Club of Alzheimer Café, samen treinen, samen vastzitten, samen rosé drinken, congressen en summerschools in de zon en de nodige dansjes, knuffels en jank- en lachattacks at the office of thuis. Samen hebben we geshaked for dementia en de kick-off gegeven voor Roermond als Dementievriendelijke gemeente. Champagne, taart en een kaartje voor mijn eerste publicatie, lieve berichtjes tijdens doctoraatdipjes en vooral heel erg veel momentjes met elkaar delen. Een betere partner-incrime had ik me niet kunnen wensen! Ik ben ontzettend trots op jou als mijn roomie en mijn vriendin en ik hoop dat ik jou straks net zo mag steunen zoals jij mij altijd hebt gesteund. Bedankt dat je mijn paranimf wilt zijn!

Lieve Anja en Joany, mijn ere-paranimfjes, jullie zijn van onbeschrijfelijke waarde voor mij geweest. Helaas zijn er regels dat er maar 2 mensen achter je mogen staan (belachelijk), maar van de andere kant ben ik ook wel heel erg blij dat ik jullie nu kan aankijken tijdens mijn verdediging omdat jullie mij bij elke stress attack weer terug naar normaal niveau wisten te brengen. Jullie waren mijn dagelijkse onuitputtelijke bron van humor, intelligentie, onnozelheid en wijsheid die ik zo ontzettend heb gewaardeerd. Niet te geloven dat ik vroeger functioneerde zonder jullie! Bedankt 
voor ALLES! Een gezamenlijk bedankje geeft jullie eigenlijk niet genoeg credits, dus wijd ik graag een paragraaf aan jullie:

Lieve An, bij jou kon ik altijd terecht: voor koffie, voor een stress-geïnduceerde lachaanval of een bezoekje aan een twijfelachtige fietsenmeneer, niets was jou te gek. Ik ben ontzettend onder de indruk van alles wat je hebt bereikt. Je bent een genie verscholen achter een knap en schattig uiterlijk, de perfecte combinatie. Nu er ook nog een kleine Anja (of Mark) bij komt, gaat de wereld er alleen maar op vooruit. Ik hoop dat we nog lang vriendinnetjes mogen blijven en mooie momenten mogen meemaken.

Lieve Joan, my sister from another mister! Omdat we soms zo ontzettend op elkaar lijken begrijp je me ook zo goed. Bij jou kan ik altijd terecht voor advies, of het nou gaat over iets wetenschappelijks of de beste haarborstel, crème, waterproof make-up of healthy recept. Ook de nodige emotionele steun kwam van jouw kant, je hebt me uitgeholpen als coach voor Partner in Balans en samen hebben we high-fivend analyses gedaan onder het genot van sushi at the office. Jij hebt ervoor gezorgd dat ik SPSS (enigszins) leuk begon te vinden. Net als jij mij dat altijd gunt, gun ik jou ook alleen maar love en happiness en ik hoop dat we nog lang 'too much information' issues met elkaar mogen delen.

Lieve Jen, tijdens de afgelopen 4 jaar was je mijn Dalai Lama! De nodige 'zen' bij ons op kantoor kwam van jou. Tegen jouw positiviteit en uitstraling kan geen psycholoog op! Lieve Liselot, bedankt voor je gezelligheid, je humor en je steun! Je hebt me vaak op stressmomentjes meegemaakt, maar was een queen in relativeren! Heel veel succes nog met je onderzoek en ik hoop dat we elkaar nog vaker blijven zien!

Nicolle en Lisanne, we hebben elkaar pas een paar keer gezien, maar wat zijn jullie top chicks! Dat we nog maar veel pitches en parties mogen rocken samen.

Alle andere (oud) collega's van de afdeling Psychiatrie en Neuropsychologie, bedankt voor al jullie hulp, gezellige samenwerking, babbeltjes en het delen van onze persoonlijke drama'tjes. Ik heb van jullie allemaal ontzettend veel geleerd. 


\section{DANKWOORD}

Mijn nieuwe collega's bij Proteion wil ik graag bedanken voor hun warme welkom! Ik ben onder de indruk van jullie passie en inlevingsvermogen en ik verheug me op een super samenwerking.

Lieve Meia, paranimf en soulmate, jij gelooft altijd meer in mij dan ik in mezelf. Je had het de afgelopen tijd zelf vaak superzwaar in je uitdagende banen, maar je wist altijd precies waar ik mee zat en steunde me door dik en dun. Bij jou kan ik altijd terecht voor een wijntje, Titanic-date, party night of gewoon een goed gesprek. Je bent een van de mooiste mensen op aarde en dat mag ook wel eens gezegd worden!

Lieve vriendinnetjes, jullie hebben mijn verhalen vaak moeten aanhoren en dat was vast niet altijd even interessant. Bedankt voor jullie luisterend oor, maar vooral ook voor de nodige afleiding en gezelligheid!

Mijn allerliefste familie en schoonfamilie, bedankt voor jullie interesse en steun tijdens deze 4 jaar en eigenlijk altijd!

Mama, mijn beste vriendinnetje! ledereen zegt altijd hoeveel ik op je lijk en daar ben ik potverdikkeme apetrots op! Samen hebben we het land doorgecrosst, gelachen, gehuild en eindeloos gekletst. Jij bent er altijd voor me! Ik heb de nodige levenslessen van je gekregen maar je hebt me vooral geleerd om te blijven lachen, ook al lacht het leven niet altijd terug. La vie est belle, hè mama! Dat we samen nog maar mogen genieten van oneindig veel zonnestralen en lunchmomentjes!

Papa, hoe druk je het ook hebt, je hebt altijd tijd en aandacht voor je kids en hun mini-drama's. Ook al wil je liever geen complimentjes ontvangen op je fantastische persoontje, je bent de beste papa die een dochter zich kan wensen! Als ik ooit mag gaan leidinggeven hoop ik dat ik jouw megadrive en innovatietalent kan voortzetten, maar vooral je menselijkheid en liefde voor je werk en familie.

Lieve grote broertjes Tom \& Nicky, bedankt dat jullie er altijd voor me zijn geweest! Nick, jij hebt zelfs de hele Zumba marathon gepresenteerd en die mastertitel was er zonder die appelflappenactie ook niet geweest! Ik ben superblij met jullie als broers 
en ben jullie ook heel dankbaar dat jullie zo'n leuke vriendinnen hebben uitgekozen, zodat ik er nu ook nog 2 topzussen bij heb gekregen!

Het laatste woordje wil ik graag gebruiken om de liefde van m'n leven te bedanken. Lieve Max, de afgelopen jaren heb je m'n drama's aangehoord, interventie-mails naar me gestuurd, relax-weekendjes ingelast, hebben we samen alle succesjes gevierd en kreeg je me altijd weer aan het lachen. Wanneer woorden tekort schoten hield je me met de nodige kilo's chocola thuis en op kantoor op de been. De laatste tijd was ik niet de gezelligste thuis, maar gelukkig heb je een interne handleiding voor dramatisch vrouwengedrag. Je bent de Barack to my Michelle en de Jay to my Bey. Dankjewel voor alles! 

CURRICULUM VITAE 


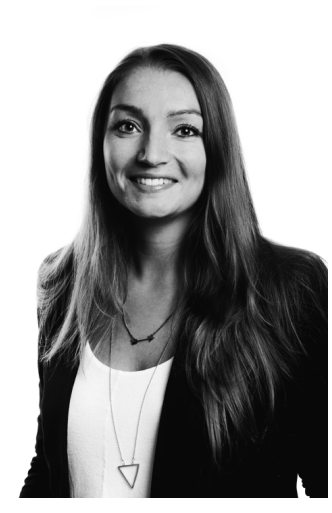

Lizzy Boots was born on December 241988 in Roermond. After graduating from high school in 2007 she obtained a bachelor degree in Health Sciences at Maastricht University in 2010. She wrote her Master thesis on the role of selfdisclosure and mimicry on likeability in social anxiety, under supervision of dr. Marisol Voncken. During her master she worked as a clinical intern at Daelzicht in Heel, where she gained experience in psychological assessment and intervention, acquiring her psychodiagnostics registration

(BAPD). After obtaining her Master degree in Mental Health at Maastricht University in 2011 (Cum Laude) she briefly worked as a care worker on outpatient basis and as a tutor at Maastricht University, before starting her $\mathrm{PhD}$ at the department of Psychiatry and Neuropsychology. Under the supervision of Prof. dr. Frans Verhey, Prof. dr. Ruud Kempen, and dr. Marjolein de Vugt she focused her research on the development, implementation, and evaluation of the innovate e-health program 'Partner in Balance' for caregivers of people with dementia. During her time as a PhD candidate she gained clinical experience in individualized and group therapy for people with mild cognitive impairment and their caregivers, was the $\mathrm{PhD}$ representative for the School of Mental Health and Neuroscience (MHeNS), organized several events in the community, and gave many lectures and talks for both scientific and lay-audiences in the Netherlands and abroad. In May of 2016, Lizzy continued her passion for supporting people with dementia and their significant others as a psychologist at Proteion in Horn. 


\section{PUBLICATIONS}


Boots LMM, de Vugt ME, van Knippenberg RJ, Kempen GIJM, Verhey FRJ (2014). A systematic review of Internet-based supportive interventions for caregivers of patients with dementia. International Journal of Geriatric Psychiatry, 29(4):331-44

Boots LMM, Wolfs CAG, Verhey FRJ, Kempen GIJM, de Vugt ME (2015). Qualitative study on needs and wishes of early-stage dementia caregivers: the paradox between needing and accepting help. International Psychogeriatrics, 27(6):927-36

Boots LMM, de Vugt ME, Withagen HEJ, Kempen GIJM, Verhey FRJ (2016). Development and initial evaluation of the web-based self-management intervention 'Partner in Balance' for family caregivers of people with early-stage dementia: an exploratory mixed-methods study. JMIR Research Protocols, 5(1):e33.

Boots LMM, de Vugt ME, Kempen GIJM, Verhey FRJ (2016). Effectiveness of the blended care self-management intervention 'Partner in Balance' for family caregivers of people with early-stage dementia: design of a randomized waiting-list controlled trial. Trials, 17(1):231

Dam AEH, Boots LMM, van Boxtel MPJ, Verhey FRJ, de Vugt ME (2016). A mismatch between demand and supply in informal dementia care: a qualitative study on perspectives of spousal caregivers and their social netwerk members. Submitted

Voncken, M., Dijk, C., Lange, WG., Boots, LMM, Roelofs, J. (2016) The role of selfdisclosure and mimicry on the development of likeability in social anxiety. Submitted

Boots, LMM, de Vugt ME, Kempen GIJM and Verhey FRJ (2016). Process evaluation to explore internal and external validity of the blended-care selfmanagement program 'Partner in Balance' for early-stage dementia caregivers. Submitted

Boots, LMM, de Vugt ME, Kempen GIJM and Verhey FRJ (2016). Effectiveness of the blended-care self-management program 'Partner in Balance' for early-stage dementia caregivers: results of a randomized controlled trial. Submitted 




\section{THESIS DEFENSES FROM MHENS}

School for Mental Health and Neuroscience 
2013

Rob Havermans: Bipolar disorder in daily life; Mood and cortisol responses to naturally occurring events. Supervisor: Prof.dr. M. de Vries; Co-Supervisor: Dr. N. Nicolson.

Véronique Moers-Hornikx: Deep brain stimulation and the cerebellum.

Supervisors: Prof.dr. J. Vles / Prof.dr. Y. Temel; Co-Supervisor: Dr. G. Hoogland.

Nicole Veldhorst-Janssen: Intranasal delivery of rapid acting drugs.

Supervisors: Prof.dr. M. Marcus / Prof.dr. C. Neef; Co-Supervisor: Dr. P.H. van der Kuy.

Stéphanie Knippenberg: Vitamin D and Multiple Sclerosis: immunological and clinical outcome. Supervisor: Prof.dr. J. Cohen-Tervaert; Co-Supervisors: Dr.

J. Damoiseaux / Dr. Y. Bols.

Erik D. Gommer: Dynamic Cerebral Autoregulation: from methodology towards clinical application. Supervisors: Prof.dr. W.H. Mess / Prof.dr. R.B. Panerai, UK; Co-Supervisor: Dr.ir. J.P.H. Reulen.

Olga A.H. Reneerkens: Can PDE inhibition improve cognition ? Translational insights. Supervisor: Prof.dr. H.W.M. Steinbusch; Co-Supervisor: Dr. J. Prickaerts;.

Lyzel S. Elias-Sonnenschein: Clinical and biomarker correlates of genetic risk factors for Alzheimer's disease. Supervisor: Prof.dr. F.R.J. Verhey; CoSupervisor: Dr. P.J. Visser.

Diego F. Mastroeni: Epigenetic Dysregulation and the Pathophysiology of of Alzheimer's Disease. Supervisors: Prof.dr. H.W.M. Steinbusch / Prof.dr. P.D. Coleman, Sun City, Arizona; Co-Supervisors: Dr. B.P.F. Rutten / Dr. D.L.A. van den Hove.

Leonidas Chouliaras: Epigenetic Regulation in Aging and Alzheimer's disease: A translational perspective. Supervisor: Prof.dr. H.W.M. Steinbusch; CoSupervisors: Dr. B.P.F. Rutten / Dr. D.L.A. van den Hove.

Liesbeth Knaepen: Perinatal events and altered pain sensitivity in later life.

Supervisors: Prof.dr. E.A.J. Joosten / Prof.dr. D. Tibboel, EUR; Co-Supervisor: Dr. J. Patijn.

Marisela Martinez-Claros: Hippocampal plasticity and corticosterone: From dendrites to behaviour. Supervisor: Prof.dr. H.W.M. Steinbusch; CoSupervisors: Dr. J.L. Pawluski / Dr. J. Prickaerts. 
Marcus D. Lancé: A circle of improvement in bleeding management: from laboratory to clinic and back. Supervisors: Prof.dr. M.A.E. Marcu / Prof.dr. J.W.M. Heemskerk; Co-Supervisor: Dr. Y.M.C. Henskens.

Hilde Braakman: Imaging the brain; neuronal correlates of cognitive impairment in children with frontal lobe epilepsy. Supervisors: Prof.dr. A.P. Aldenkamp / Prof.dr. J.S.H. Vles; Co-Supervisors: Dr.ir. W.H. Backes / Dr. P.A.M. Hofman. Willem $\mathrm{H}$. van Zwam: Aneurysmal subarachnoid hemorrhage: imaging strategies and cost-effectiveness aspects in diagnostic work-up and posttherapeutic follow-up. Supervisors: Prof.dr. J.T. Wilmink / Prof.dr. J.E. Wildberger; Co-Supervisor: Dr. P.A.M. Hofman.

Klara De Cort: The Pathogenesis of Panic Disorder. Supervisors: Prof.dr. I. MyinGermeys / Prof.dr. E.J.L. Griez; Co-Supervisors: Dr. K.R.J. Schruers / Dr. I. Van Diest, Leuven.

Kim van Wijck: Mind the Gap; experimental studies on splanchnic hyperfusion and gastrointestinal integrity loss in man. Supervisors: Prof.dr. W.A. Buurman / Prof.dr. C.H.C. Dejong; Co-Supervisor: Dr. K. Lenaerts.

Yvette Roke: Antipsychotic-induced hyperprolactinemia in children and adolescents with mainly autism spectrum disorders. Prevalence, symptoms, clinical consequences and genetic risk factors. Supervisors: Prof.dr. P.N. van Harten / Prof.dr. J.K. Buitelaar (RUN); Co-Supervisor: Dr. A. Boot (UMCG).

Fleur Goezinne: Retinal detachment surgery: pre and postoperative prognostic factors. Supervisors: Prof.dr. F. Hendrikse / Prof.dr. C.A.B. Webers; CoSupervisor: Dr. E.C. La Heij (Amsterdam).

Ralph L.J.G. Maassen: The Merits of Videolaryngoscopy during Glottic Visualisation for Endotracheal Intubation. Supervisors: Prof.dr. M. Marcus / Prof.dr. A. van Zundert (University of Queensland).

Maria J. de Sousa Guerreiro: The role of sensory modality in age-related distraction. Supervisor: Prof.dr. C.M. van Heugten; Co-Supervisor: Dr. P.W.M. van Gerven.

Ine Rayen: Effects of developmental fluoxetine exposure on neurobehavioral outcomes. Supervisor: Prof.dr. H.W.M. Steinbusch; Co-Supervisors: Dr. J.L. Pawluski /

Dr. T.D. Charlier (Ohio University, USA).

Nynke M.G. Bodde: Psychogenic non-epileptic seizures; a separate disorder or 
part of a continuum? Supervisors: Prof.dr. R. van Oostenbrugge / Prof.dr. K. Vonck (UZ Gent); Co-Supervisors: Dr. R. Lazeron / Dr. A. de Louw (Epilepsiecentrum Kempenhaeghe, Heeze).

Alejandro M. Gomez: Novel strategies for making myasthenia less gravis: targeting plasma cells and the neuromuscular junction. Supervisor: Prof.dr. M.H. De Baets; Co-Supervisors: Dr. M. Losen / Dr. P. Martinez-Martinez. Mohammad S. Rahnama'i: Prostaglandins and Phosphodiesterases in the Urinary Bladder Wall. Supervisors: Prof.dr. Ph. Van Kerrebroeck / Prof.dr. S. de Wachter (Universiteit Antwerpen); Co-Supervisor: Dr. G. van Koeveringe.

Mariken B. de Koning: Studying biomarkers in populations at genetic and clinical high risk for psychosis. Supervisors: Prof.dr. T. Amelsvoort / Prof.dr. J. Booij (AMC).

Fabien Boulle: Epigenetic regulation of BDNF/TrkB signaling in the pathophysiology and treatment of mood disorders. Supervisors: Prof.dr. H.W.M. Steinbusch / Prof.dr. L. Lanfumey (Universiteit Parijs); Co-Supervisors: Dr. D. van den Hove / Dr. G. Kenis.

2014

Iris Nowak-Maes: Tinnitus; assessment of quality of life \& cost-effectiveness.

Supervisors: Prof.dr. M. Peters / Prof.dr. B. Kremer; Co-Supervisors: Dr. M. Joore /

Dr. L. Anteunis.

Marjolein Huijts: Cognitive function in patients with cerebral small vessel disease. Supervisor: Prof.dr. R.J. van Oostenbrugge; Co-Supervisors: Dr. A.A. Duits / Dr. J. Staals.

Markus Gantert: Fetal inflammatory injury as origin of long term disease:

Lessons from animal models. Supervisors: Prof.dr. B. Kramer / Prof.dr. L. Zimmermann; Co-Supervisor: Dr. A. Gavilanes.

Elke Kuypers: Fetal development after antenatal exposures: Chorioamnionitis and maternal glucocorticoids. Supervisors: Prof.dr. B.W. Kramer / Prof.dr. H.W. Steinbusch / Prof.dr. Suhas G. Kallapur (University of Cincinnati, Ohio, USA).

Pieter Kubben: Ultra low-field strength intraoperative MRI for Glioblastoma Surgery. Supervisor: Prof.dr. J.J. van Overbeeke; Co-Supervisor: Dr. H. van Santbrink. 
Laura Baijens: Surface electrical stimulation of the neck for oropharyngeal dysphagia in Parkinson's disease: therapeutic aspects and reliability of measurement. Supervisor: Prof.dr. B. Kremer; Co-Supervisor: Dr. R. Speyer, Townsville.

Janneke Hoeijmakers: Small fiber neuropathy and sodium channels; a paradigm shift. Supervisor: Prof.dr. R.J. van Oostenbrugge; Co-Supervisors: Dr. C.G. Faber / Dr. I.S.J. Merkies.

Stephanie Vos: The Role of biomarkers in preclinical and prodromal Alzheimer's disease. Supervisor: Prof.dr. F.R. Verhey; Co-Supervisor: Dr. P.J. Visser. Muriël Doors: The Value of Optical Coherence Tomography in Anterior Segment Surgery. Supervisors: Prof.dr. R.M. Nuijts / Prof.dr. C.A. Webers; CoSupervisor: Dr. T.T.J.M. Berendschot.

Anneke Maas: Sleep problems in individuals with genetic disorders associated with intellectual disability. Supervisors: Prof.dr. I. Curfs / Prof.dr. R. Didden. Sebastiaan van Gorp: Translational research on spinal cord injury and cellbased therapies; a focus on pain and sensorimotor disturbances. Supervisors: Prof.dr. B. Joosten / Prof.dr. M. van Kleef; Co-Supervisors: Dr. J. Patijn /Dr. R. Deumens, KU Leuven.

Andrea Sannia: High risk newborns and brain biochemical monitoring.

Supervisor: Prof.dr. J.S.H. Vles; Co-Supervisors: Dr. D. Gazzolo, Alessandria, Italy / Dr. A.W.D. Gavilanes.

Julie A.D.A. Dela Cruz: Dopamine mechanisms in learning and memory:

Evidence from rodent studies. Supervisors: Prof.dr. H.W.M. Steinbusch /

Prof.dr. R.J. Bodnar, New York; Co-Supervisor: Dr. B.P.F. Rutten.

René Besseling: Brain wiring and neuronal dynamics; advances in MR imaging of focal epilepsy. Supervisors: Prof.dr. A.P. Aldenkamp / Prof.dr.ir. W.H.

Backes; Co-Supervisor: dr. J.F.A. Jansen.

Maria Quint-Fens: Long-term care after stroke; development and evaluation of a long-term intervention in primary care. Supervisors: Prof.dr. J.F.M.

Metsemakers / Prof.dr. C.M. van Heugten / Prof.dr. M. Limburg, Almere; CoSupervisor: dr. G.H.M.I. Beusmans.

Veronique Moulaert: Life after survival of a cardiac arrest; the heart of the matter. Supervisors: Prof.dr. J.A. Verbunt / Prof.dr. C.M. van Heugten / Prof.dr. D.T. Wade, Oxford, UK.

Feikje Smeets: The hallucinatory-delusional state: a crucial connection in the 
psychosis symptom network. Supervisor: Prof.dr. J. van Os; Co-Supervisor: Dr. T. Lataster.

Lies Clerx: Alzheimer's disease through the MR-eye; novel diagnostic markers and the road to clinical implementation". Supervisor: Prof.dr. F. Verhey; CoSupervisors: Dr. P.J. Visser / P. Aalten.

Sonny Tan: The subthalamic nucleus in Parkinson's disease. Supervisors:

Prof.dr. Y. Temel / Prof.dr. H.W.M. Steinbusch / Prof.dr. T. Sharp, Oxford, UK / Prof.dr. V. Visser-Vandewalle, Koln.

Koen van Boxem: The use of pulsed radiofrequency in the management of chronic lumbosacral radicular pain. Supervisors: Prof.dr. M. van Kleef / Prof.dr. E.A.J. Joosten; Co-Supervisor: Assoc. Prof.dr. J. van Zundert.

Jérôme Waterval: Hyperostosis cranialis interna. Supervisors: Prof.dr. J.J. Manni /

Prof.dr. R.J. Stokroos.

Sylvie Kolfschoten-van der Kruijs: Psychogenic non-epileptic seizures; the identification of neurophysiological correlates. Supervisors: Prof.dr. A.P.

Aldenkamp / Prof.dr. K.E.J. Vonck, Universiteit Gent; Co-Supervisors: Dr. J.F.A. Jansen / Dr. R.H.C. Lazeron, Kempenhaeghe.

Wouter Pluijms: Spinal cord stimulation and pain relief in painful diabetic:

polyneuropathy, a translational approach. Supervisors: Prof.dr. M. van Kleef / Prof.dr. E.A. Joosten; Co-supervisor: Dr. C.G. Faber.

Ron Handels: Health technology assessment of diagnostic strategies for

Alzheimer's disease. Supervisors: Prof.dr. F.R.J. Verhey / Prof.dr. J.L.

Severens (EUR); Co-Supervisor: Dr. M.A. Joore / Dr. C.A.G. Wolfs.

Evelyn Peelen: Regulatory T cells in the pathogenesis of Multiple Sclerosis:

potential targets for vitamin D therapy. Supervisors: Prof.dr. R.M.M.

Hupperts / Prof.dr. J.W. Cohen Tervaert; Co-Supervisor: Dr. J.G.M.C.

Damoiseaux / Dr. M.M.G.L.Thewissen, Diepenbeek.

Reint Jellema: Cell-based therapy for hypoxic-ischemic injury in the preterm brain. Supervisors: Prof.dr. B.W.W. Kramer / Prof.dr. H.W.M. Steinbusch; CoSupervisor: Dr. W.T.V. Germeraad / Dr. P. Andriessen, Veldhoven.

Maria Wertli: Prognosis of Chronic Clinical Pain Conditions: The Example of Complex Regional Pain Syndrome 1 and Low Back Pain. Supervisors: Prof.dr. M. van Kleef; Co-Supervisor: Dr. F. Brunner, Zürich / Dr. R. Perez, VUmc.

Dagmar Zeef: An experimental model of Huntington's disease: Validation \& 
Stimulation. Supervisors: Prof.dr. Y. Temel / Prof.dr. H.W.M. Steinbusch; Cosupervisor: Dr. A. Jahanshahi.

Jeroen Decoster: Breaking Down Schizophrenia into phenes, genes and environment. Supervisors: Prof.dr. I. Myin-Germeys / Prof.dr. M. De Hert, KU Leuven; Co-Supervisor: Dr. R. van Winkel.

Eaja Anindya Sekhar Mukherjee: Fetal Alcohol Spectrum Disorders: exploring prevention and management. Supervisor: Prof.dr. L.M.G. Curfs; Co-

Supervisor: Prof. S. Hollins, St. George's University of London, UK.

Catherine van Zelst: Inside out; On stereotype awareness, childhood trauma and stigma in psychosis. Supervisors: Prof.dr. Ph. Delespaul / Prof.dr. J. van Os. Ibrahim Tolga Binbay: Extended Psychosis Phenotype in the Wider Social

Environment. Supervisor: Prof.dr. J. van Os; Co-Supervisor: Dr. M. Drukker. Frank Van Dael: OCD matters in psychosis. Supervisors: Prof.dr. J. van Os /

Prof.dr. I. Myin-Germeys.

Pamela Kleikers: NOXious oxidative stress: from head toe too and back.

Supervisors: Prof.dr. H.H.H.W. Schmidt / Prof.dr. H.W.M. Steinbusch; Co-

Supervisor: Dr. B. Janssen.

José Luis Gerardo Nava: In vitro assay systems in the development of therapeutic interventions strategies for neuroprotection and repair.

Supervisors: Prof.dr.med. J. Weis / Prof.dr. H.W.M. Steinbusch; Co-Supervisor:

Dr. G.A. Brook, RWTH Aachen.

Eva Bollen: Cyclic nucleotide signaling and plasticity. Supervisors:

Prof.dr. H.W.M. Steinbusch / Prof.dr. R. D'Hooge, KU Leuven; Co-Supervisor: Dr. J. Prickaerts.

2015

Jessica A. Hartmann: A good laugh and a long sleep; Insights from prospective and ambulatory assessments about the importance of positive affect and sleep in mental health. Supervisor: Prof.dr. J. van Os; Co-Supervisors: C.J.P. Simons / Dr. M. Wichers.

Bart Ament: Frailty in old age; conceptualization and care innovations.

Supervisors: Prof.dr. G.I.J.M. Kempen / Prof.dr. F.R.J. Verhey; Co-Supervisor: Dr. M.E. de Vugt.

Mayke Janssens: Exploring course and outcome across the psychosiscontinuum. Supervisor: Prof.dr. I. Myin-Germeys; Co-Supervisor: Dr. T. 
Lataster

Dennis M.J. Hernau: Dopayours is not dopamine: genetic, environmental and pathological variations in dopaminergic stress processing. Supervisor:

Prof.dr. I. Myin-Germeys; Co-Supervisors: Prof.dr. F.M. Mottaghy / Dr. D. Collip. Ingrid M.H. Brands: The adaptation process after acquired brain injury Pieces of

the puzzle. Supervisors: Prof.dr. C.M. van Heugten / Prof.dr. D.T. Wade,

Oxford UK; Co-Supervisors: Dr. S.Z. Stapert / Dr. S. Köhler.

Francesco Risso: Urinary and salivary S100B monitoring in high risk infants.

Supervisor: Prof.dr. J.S.H. Vles; Co-Supervisors: Dr. D. Gazzolo, Genoa,Italy /

Dr. A.W.D. Gavilanes.

Alessandro Borghesi: Stem and Progenitor Cells in Preterm Infants: Role in the

Pathogenesis and Potential for Therapy. Supervisor: Prof.dr. L.

Zimmermann; Prof.dr. B. Kramer; Co-Supervisors: Dr. D. Gazzolo, Genoa,Italy /

Dr. A.W.D. Gavilanes.

Claudia Menne-Lothmann: Affect dynamics; A focus on genes, stress, and an

opportunity for change. Supervisor: Prof.dr. J. van Os; Co-Supervisors: Dr. M.

Wichers / Dr. N. Jacobs.

Martine van Nierop: Surviving childhood new perspectives on the link between

childhood trauma and psychosis. Supervisors: Prof.dr. I. Myin-Germeys /

Prof.dr. J. van Os; Co-Supervisor: Dr. R. van Winkel.

Sylvia Klinkenberg: VNS in children; more than just seizure reduction.

Supervisors: Prof.dr. J. Vles / Prof.dr. A. Aldenkamp; Co-Supervisor: Dr. H. Majoie.

Anouk Linssen: Considerations in designing an adult hearing screening

programme. Supervisor: Prof.dr. B. Kremer; Co-Supervisors: Dr. L. Anteunis /

Dr. M. Joore.

Janny Hof: Hearing loss in young children; challenges in assessment and

intervention. Supervisors: Prof.dr. B. Kremer / Prof.dr. R. Stokroos / Prof.dr. P. van Dijk, RUG;

Co-Supervisor: Dr. L. Antheunis.

Kimberly Cox-Limpens: Mechanisms of endogenous brain protection; Clues

from the transcriptome. Supervisors: Prof.dr. J. Vles / Prof.dr. L.

Zimmermann; Co-Supervisor:

Dr. A. Gavilanes.

Els Vanhoutte: Peripheral Neuropathy outcome measures; Standardisation 
(PeriNomS) study part 2: Getting consensus. Supervisors: Prof.dr. C. Faber / Prof.dr. P. van Doorn;

Co-Supervisor: Dr. I. Merkies, Spaarne ziekenhuis Hoofddorp.

Mayienne Bakkers: Small fibers, big troubles; diagnosis and implications of small fiber neuropathy. Supervisors: Prof.dr. C. Faber / Prof.dr. M. de Baets;

Co-Supervisor: Dr. I. Merkies, Spaarne ziekenhuis Hoofddorp.

Ingrid Kramer: Zooming into the micro-level of experience: An approach for understanding and treating psychopathology. Supervisor: Prof.dr. J. van Os; Co-Supervisors: Dr. M. Wichers, UMC Groningen / Dr. C. Simons.

Esther Bouman: Risks and Benefits of Regional Anesthesia in the Perioperative Setting. Supervisors: Prof.dr. M. van Kleef / Prof.dr. M. Marcus, HMC, Qatar / Prof.dr. E. Joosten; Co-Supervisor: Dr. H. Gramke.

Mark Janssen: Selective stimulation of the subthalamic nucleus in Parkinson's disease; dream or near future. Supervisors: Prof.dr. Y. Temel / Prof.dr. V. Visser-Vandewalle, Keulen / Prof.dr. A. Benazzouz, Bordeax, France.

Reina de Kinderen: Health Technology Assessment in Epilepsy; economic evaluations and preference studies. Supervisors: Prof.dr. S. Evers / Prof.dr.

A. Aldenkamp; Co-Supervisor: Dr. H. Majoie / Dr. D. Postulart, GGZ O-Brabant. Saskia Ebus: Interictal epileptiform activity as a marker for clinical outcome.

Supervisors: Prof.dr. A. Aldenkamp / Prof.dr. J. Arends, TUE / Prof.dr. P. Boon, Universiteit Gent, België.

Inge Knuts: Experimental and clinical studies into determinants of panic severity. Supervisor: Prof.dr. I. Myin-Germeys; Co-Supervisor: Dr. K. Schruers; Influencing panic.

Nienke Tielemans: Proactive coping post stroke: The Restored4Stroke Self-

Management study. Supervisors: Prof.dr. C. van Heugten / Prof.dr. J. VisserMeily, UMC Utrecht;

Co-Supervisor: Dr. V. Schepers, UMC Utrecht.

Tom van Zundert: Improvements Towards Safer Extraglottic Airway Devices.

Supervisors: Prof.dr. A.E.M. Marcus / Prof.dr. W. Buhre / Prof.dr. J.R.

Brimacombe, Queensland, Australia / Prof.dr. C.A. Hagberg.

Tijmen van Assen: Anterior Cutaneous Nerve Entrapment Syndrome

Epidemiology and surgical management. Supervisors: Prof.dr. G.L. Beets /

Prof.dr. M. van Kleef / Dr. R.M.H. Roumen / Dr. M.R.M. Scheltinga, MMC Veldhoven. 
Rohit Shetty: Understanding the Clinical, Immunological and Genetic Molecular Mechanisms of Keratoconus. Supervisors: Prof.dr. R.M.M.A. Nuijts / Prof.dr. C.A.B. Webers.

Christine van der Leeuw: Blood, bones and brains; peripheral biological endophenotypes and their structural cerebral correlates in psychotic disorder. Supervisor: Prof.dr. J. van Os; Co-supervisor: Dr. M. Marcelis.

Sanne Peeters: The Idle Mind Never Rests; functional brain connectivity across the psychosis continuum. Supervisor: Prof.dr. J. van Os; Co-supervisor: dr. M. Marcelis.

Nick van Goethem: $\boldsymbol{\alpha} 7$ nicotinic acetylcholine receptors and memory processes: mechanistic and behavioral studies. Supervisor: Prof.dr. H.W.M. Steinbusch; Co-supervisor: Dr. J. Prickaerts.

Nicole Leibold: A Breath of fear; a translational approach into the mechanisms of panic. Supervisor: Prof.dr. H.W.M. Steinbusch; Co-supervisors: Dr. K.R.J. Schruers / Dr. D.L.A. van den Hove.

Renske Hamel: The course of mild cognitive impairment and the role of comorbidity. Supervisor: Prof.dr. F.R.J. Verhey; Co-supervisors: Dr. I.H.G.B. Ramakers / Dr. P.J. Visser.

Lucia Speth: Effects of botulinum toxin A injections and bimanual task-oriented therapy on hand functions and bimanual activities in unilateral Cerebral Palsy. Supervisors: Prof.dr. J. Vles; Prof.dr. R. Smeets; Co-supervisor: Dr. Y. Janssen-Potten, Adelante Hoensbroek.

Yuan Tian: The effects of Lutein on the inflammatory pathways in age-related macular degeneration (AMD). Supervisors: Prof.dr. C. Webers; Prof.dr. A. Kijlstra, WUR; Co-supervisor: Dr. M. Spreeuwenberg; Dr. H. Tange.

Peggy Spauwen: Cognition and Type 2 diabetes; the interplay of risk factors. Supervisors: Prof.dr. F. Verhey; Prof.dr. C. Stehouwer; Co-supervisor: Dr. M. van Boxtel

Marc Hilhorst: Crescentic glomerulonephritis in ANCA associated vasculitis. Supervisors: Prof.dr. J. Cohen-Tervaert; Co-supervisor: Dr. P. van Paassen Martin Gevonden: The odd one out: exploring the nature of the association between minority status and psychosis. Supervisors: Prof.dr. J-P. Selten; Prof.dr. J. Booij, Uva; Prof.dr. I. Myin-Germeys

Bart Biallosterski: Structural and functional aspects of sensory-motor Interaction in the urinary bladder. Supervisors: Prof.dr. Ph. Van Kerrebroeck; Prof.dr. S. 
De Wachter, UvAntwerpen; Co-supervisors: Dr. G. van Koeveringe; Dr. M. Rahnama'i.

Alexandra König: The use of information and communication technologies (ICT) for the assessment of patients with Alzheimer's Disease and related disorders. Supervisors: prof.dr. F. Verhey; prof.dr. Ph. Robert, Nice, Fr; Cosupervisors: dr. P. Aalten; dr. R. David, Nice. Fr.

Michelene Chenault: Assessing Readiness for Hearing Rehabilitation.

Supervisors: prof.dr. M.P.F. Berger; prof.dr. B. Kremer; Co-supervisor: dr. L.J.C. Anteunis.

Anand Vinekar: Retinopathy of Prematurity. Recent advances in tele-medicine screening, risk factors and spectral domain optical coherence tomography imaging. Supervisor: prof.dr. C.A.B. Webers; Co-supervisor: dr. N.J. Bauer

Fleur van Dooren: Diabetes and Depression: exploring the Interface between Pathophysiological and Psychological factors. Supervisors: prof.dr. F.R.J. Verhey; prof.dr. J.K.L. Denollet, UvT; prof.dr. F. Pouwer, UvT; Co-supervisor: dr. M.T. Schram.

Gabriëlla Pons van Dijk: Taekwondo and physical fitness components in middleaged healthy volunteers; the Sekwondo study. Supervisors: prof.dr. J. Lodder; prof.dr. H. Kingma; Co-supervisor: dr. A.F. Lenssen.

Yara Pujol López: Development and psychoneuroimmunological mechanisms in depression. Supervisor: prof.dr. H.W.M. Steinbusch; Co-supervisors: Dr. G. Kenis; Dr. D. van den Hove; Dr. Aye Mu Myint, München.

Romina Gentier: UBB ${ }^{+1}$; an important switch in the onset of Alzheimer's disease.Supervisors: Prof. H. Steinbusch; Prof. D. Hopkins; Co-supervisor: Dr. F. van Leeuwen.

Sanne Smeets: Insights into insight: studies on awareness of deficits after acquired brain injury. Supervisor: Prof. C. van Heugten; Prof. R. Ponds; Cosupervisor: Dr. I. Winkens

Kim Beerhorst: Bone disease in chronic epilepsy: fit for a fracture. Supervisor:

Prof. A. Aldenkamp; Prof. R. van Oostenbrugge; Co-supervisor: Dr. P. Verschuure.

Alex Zwanenburg: Cerebral and cardiac signal monitoring in fetal sheep with hypoxic-ischemic encephalopathy. Supervisor: Prof. T. Delhaas; Prof. B. Kramer; Co-supervisors: Dr. T. Wolfs; Dr. P. Andriessen, MMC. 
Ismail Sinan Guloksuz: Biological mechanisms of environmental stressorsin psychiatry. Supervisor: Prof. J. van Os; Co-supervisors: Dr. B. Rutten; Dr. M. Drukker.

Seyed Ehsan Pishva MD: Environmental Epigenetics in mental health and

illness. Supervisor: Prof.dr. J. van Os; Co-supervisors: Dr. B.P.F. Rutten; Dr.

G. Kenis.

Ankie Hamaekers: Rescue ventilation using expiratory ventilation assistance;

innovating while clutching at straws. Supervisors: Prof.dr. W.F. Buhre;

Prof.dr. M. van Kleef.

Rens Evers. 22q11.2 deletion syndrome: intelligence, psychopathology and neurochemistry at adult age. Supervisors: Prof.dr. L.M.G. Curfs; Prof.dr. T. v. Amelsvoort.

Sarah-Anna Hescham. Novel insights towards memory restoration. Supervisor:

Prof.dr.

Y. Temel; Co-supervisor: Dr. A. Blokland; Dr. A. Jahanshahi.

João P. da Costa Alvares Viegas Nunes. Insulin receptor sensitization improves affective pathology in various mouse models. Supervisor: Prof.dr. H.W.M. Steinbusch; Co-supervisors: Dr. K-P. Lesch; Dr. T. Strekalova; Dr.B.H. Cline, Oxford.

Yanny Ying-Yee Cheng. Clinical Outcomes After Innovative Lamellar Corneal

Transplantation Surgery. Supervisor: Prof.dr. R.M.M.A. Nuijts; Co-supervisor: Dr. J.S.A.G. Schouten.

2016

Oliver Gerlach. Parkinson's disease, deterioration during hospitalization.

Supervisor: Prof.dr. R. van Oostenbrugge; Co-supervisor: Dr. W. Weber. Remo Arts. Intracochlear electrical stimulation to suppress tinnitus.

Supervisor: Prof.dr. R.J. Stokroos; Co-supervisor: Dr. E.L.J. Georg. Mitchel van Eeden. The $€$ - Restore4stroke study: Economic evaluation of stroke care in the Netherlands. Supervisors: Prof.dr.mr. S.M.A.A. Evers; Prof.dr. C.M. v. Heugten; Co-supervisor: dr. G.A.P. van Mastrigt.

Pim Klarenbeek. Blood pressure and cerebral small vessel disease. Supervisor:

Prof.dr. R.J. van Oostenbrugge; Co-supervisor: Dr. J. Staals. 
Ramona Hohnen. Peripheral pharmacological targets to modify bladder contractility. Supervisor: Prof.dr. Ph.E.V. van Kerrebroeck; Co-supervisors: Dr. G.A. van Koeveringe; Dr. M.A. Sahnama'i; Dr. C. Meriaux.

Ersoy Kocabicak. Deep brain stimulation of the subthalamic nucleus: Clinical and scientific aspects. Supervisors: Prof.dr. Y. Temel; Prof.dr. K. van Overbeeke; Co-supervisor: Dr. A. Jahanshahi.

Sven Akkerman. Temporal aspects of cyclic messenger signaling in object recognition memory; a pharmalogical approach. Supervisor: Prof.dr. H.W.M. Steinbusch; Co-supervisors: dr. J. Prickaerts; dr. A. Blokland.

Anja Moonen. Emotion and Cognition in Parkinson's disease; etiology and neurobiological mechanisms. Supervisor: Prof.dr. F.R.J. Verhey; Cosupervisor: dr. A.F.G. Leentjens.

Anna Schüth. Three-dimensional bladder tissue morphology. Supervisors: Prof.dr. G.A. van Koeveringe; Prof.dr. M. v. Zandvoort, Aachen; Prof.dr. Ph. V. Kerrebroeck.

Elisabeth van der Ven. Ethnic minority position as risk indicator for autismSpectrum and psychotic disorders. Supervisors: Prof.dr. J.P. Selten; Prof.dr. J. van Os.

Zuzana Kasanova. Environmental reactivity for better or worse; The impact of stress and reward on neurochemistry, affect and behavior across the psychosis continuum. Supervisor: Prof.dr. I. Myin-Germeys, KU Leuven/UM; Co supervisor: dr. D. Collip. 
